\title{
Quantum quench dynamics in the transverse field Ising model of fully connected spins
}

An entanglement based study beyond mean field

\author{
Dissertation \\ for the award of the degree \\ "Doctor rerum naturalium" (Dr. rer. nat.) \\ of the Georg-August-Universität Göttingen \\ within the doctoral program physics \\ of the Georg-August University School of Science (GAUSS)
}

submitted by

Ingo Homrighausen

from Siegen

Göttingen, 2020 


\title{
Thesis Committee
}

- Prof. Dr. Stefan Kehrein

Condensed Matter Theory, Institute for Theoretical Physics

- Prof. Dr. Fabian Heidrich-Meisner

Condensed Matter Theory, Institute for Theoretical Physics

- PD Dr. Salvatore Manmana

Condensed Matter Theory, Institute for Theoretical Physics

\section{Members of the Examination Board}

\author{
Reviewer: $\quad$ Prof. Dr. Stefan Kehrein, \\ Condensed Matter Theory, Institute for Theoretical Physics \\ Second Reviewer: Prof. Dr. Fabian Heidrich-Meisner \\ Condensed Matter Theory, Institute for Theoretical Physics
}

\section{Further Members of the Examination Board}

- PD Dr. Salvatore Manmana

Condensed Matter Theory, Institute for Theoretical Physics

- PD Dr. Martin Wenderoth

IV. Physical Institute

- Prof. Dr. Reiner Kree

Complex Systems, Institute for Theoretical Physics

- Prof. Dr. Peter Sollich

Non-equilibrium Statistical Physics, Institute for Theoretical Physics

Date of the oral examination: April 21, 2020 


\section{Acknowledgements}

Foremost, I thank Stefan Kehrein for the possibility to write my dissertation along with scientific publications in his research group. His supervision over many years was always very supportive, reliable, and empathetic. Numerous valuable scientific discussions with Stefan Kehrein have shaped my understanding, part of which has condensed into this treatise. I am grateful that I had the opportunity to visit several national and international conferences (amongst others two times the APS March Meeting) and workshops, as well as a summer school on "Non-linear Dynamics, Dynamical Transitions and Instabilities in Classical and Quantum Systems" at the ICTP in Trieste.

Next, I would like to thank Fabian Heidrich-Meisner for being the second reviewer, as well as Salvatore Manmana, Martin Wenderoth, Peter Sollich, and Reiner Kree for taking part in the examination board.

I acknowledge careful proofreading by Salvatore Manmana, Stefan Kehrein, Alex Osterkorn, and Niklas Bölter.

I thank Nils Abeling, Jad Halimeh, and Valentin Zauner-Stauber for the friendly and motivating collaboration resulting in a publication. I also enjoyed the joint work and discussions on the quantum Kac ring 1 with Johannes Oberreuter.

Furthermore, I acknowledge scientific and non-scientific discussions with Johannes Oberreuter, Mariya Medvedyeva, Salvatore Manmana, Fabian Biebl, Ebad Kamil, Benjamin Lenz, Pei Wang, Hannes Kriel, Nils Abeling, Markus Schmitt, Niklas Bölter, Alex Osterkorn, Kristof Harms, Tridev Mishra, Mirsolav Hopjan, Gaoyuan Wang, Jan Veltmaat, Jelger Risselada, Yulia Smirnova, Veronica Chappa, and many more, who have contributed to a lively and motivating atmosphere at the institute.

I feel particularly privileged to thank Gaoyuan Wang for her crucial encouragements, wise advices, and helpful honest feedback. I thank David, Regine, Alma, and Simon Jahn for their friendly hospitality and support.

Last, but not least, I want to thank my parents Waltraud and Jürgen Homrighausen for continuous support, as well Vera Homrighausen, Ingrid Stähler, Manuel Wiese, Sonja Esch, Stefan Ignorek, Ji Liu, Raphael Köhler, Bernadette Lessel, Hans Weiss, and Yujie Li for their reliable help and joint pleasant times.

\footnotetext{
${ }^{1}$ Johannes M. Oberreuter, Ingo Homrighausen, Stefan Kehrein, Entanglement propagation and typicality of measurements in the quantum Kac ring, Annals of Physics, Volume 348, 2014
} 
Acknowledgements 


\section{Abstract}

The transverse field Ising model of $N$ fully connected spins provides a mathematical accessible, yet non-trivial, system to investigate the non-thermal dynamics after a sudden quantum quench. The focus in this thesis of three-fold.

First, in a $1 / N$ expansion the dynamics of the mean magnetization within its variance is obtained analytically in the large $N$ limit. The variance constitutes a leading order correction to the mean field limit at infinite $N$. While mean field theories are one of the most accessible approximations to deal with the quantum complexity, its validity in time out of equilibrium has to be checked on a case by case basis. By studying the dynamics of the variance, we see that the mean field approximation can break down at surprisingly early times scaling with the square root of $N$. The underlying mechanism in the fully connected Ising model is identified as a dephasing effect between nearby effective orbits. The dynamics of the magnetization variance discriminates four qualitatively different regions in the dynamical phase diagram that are indistinguishable by just looking at the mean magnetization.

Second, bipartite entanglement between disjoint groups of the spins in the fully connected Ising model is studied. In the large $N$ limit the entanglement Hamiltonian is shown to be a harmonic oscillator. A quantitative relation between the angular frequency of the oscillator and a squeezing measure of the spin state is derived. Explicit expressions for the Rényi entanglement entropies are given. In contrast to the variance, which vanishes in the thermodynamic limit, the entanglement entropy saturate to an $N$-independent positive value. The dynamics of the entanglement Hamiltonian and the entanglement entropies is discussed. Linear growth as a function of time is linked to the unstable fixed point and the homoclinic orbit and of a pitchfork bifurcation, while logarithmic increase is explained due to a dephasing mechanism of nearby effective orbits. A generalization to other models that permit a semiclassical effective description (on a possibly higher dimensional phase space) is given. As a byproduct the connection between entangled Gaussian states and the symplectic eigenvalues of its reduced covariance is derived. An upper bound on the von Neumann entanglement entropy of any bipartite pure state is given in terms of the symplectic properties of its reduced covariance.

Third, the existence of dynamical phase transitions (DPTs) in the fully connected Ising model is argued by two different approaches. DPTs are defined as non-analyticities in the time-dependence of the return probability rate function in the thermodynamic limit. Like entanglement entropy, the behavior of the return rate function is an observable-independent quantity used to characterize non-equilibrium states. A numerically based connection between the DPT and the analytic property of the infinite time averaged order parameter as a function of quench strength is discussed.

The concept of Feynman history states is used to devise the notion of history entanglement. An information theoretic interpretation of the history entanglement entropy as the precision of a quantum clock follows from Holevo's bound. The dependence of this entanglement entropy on the observation time constitutes an operator-independent characterization of the quantum dynamics in closed systems. For observation times comparable to the Heisenberg time, the history entanglement becomes sensitive to the statistics of the energy gaps, and thus distinguishes integrable and nonintegrable dynamics. This is demonstrated for two examples, a random matrix ensemble, and a onedimensional hardcore boson model with integrability breaking next nearest neighbor interaction. 
Abstract 


\section{List of symbols}

\begin{tabular}{|c|c|}
\hline$N$ & system size, number of elementary spins \\
\hline $\mathcal{H}_{N}=\otimes^{N} \mathbb{C}^{2}$ & Hilbert space of $N$ spins \\
\hline $\mathcal{D}_{N} \cong l^{2}\left(\mathbb{Z}_{N+1}\right)$ & Dicke space of $N$ spins \\
\hline $\mathcal{H}_{\mathrm{eff}} \cong L^{2}(0,1)$ & Hilbert space for effective description as $N \rightarrow \infty$ \\
\hline$\left(\sigma_{i}^{x}, \sigma_{i}^{y}, \sigma_{i}^{z}\right)$ & Pauli spin operators of $i$-th elementary spin \\
\hline $\mathbf{S}=\sum_{i}^{N}\left(\sigma_{i}^{x}, \sigma_{i}^{y}, \sigma_{i}^{z}\right) / 2$ & collective spin operators \\
\hline $\mathbf{s}=\frac{1}{N} \sum_{i}^{N}\left(\sigma_{i}^{x}, \sigma_{i}^{y}, \sigma_{i}^{z}\right) / 2$ & intensive collective spin operators \\
\hline$H$ & fully connected transverse field Ising Hamiltonian \\
\hline$H_{\text {eff }}$ & effective Hamiltonian \\
\hline$\Gamma$ & transverse magnetic field \\
\hline $\mathrm{H}_{2}$ & binary Shannon entropy \\
\hline$S_{n}=\frac{1}{1-n} \log \operatorname{Tr} \rho^{n}$ & $n$-th Rényi entropy of density matrix $\rho$ \\
\hline$S_{\mathrm{vN}}=-\operatorname{Tr} \rho \log \rho$ & von Neumann entropy of density matrix $\rho$ \\
\hline$f$ & rate function \\
\hline$f_{2}$ & curvature (second derivative) of the rate function $f$ \\
\hline$g_{2}$ & real part of $f_{2}$ \\
\hline$\theta_{2}$ & negative imaginary part of $f_{2}$ \\
\hline$n$ & configuration space dimension \\
\hline $\mathbf{z}=(\mathbf{x}, \mathbf{p})$ & $2 n$ dimensional phase space coordinates \\
\hline$\nabla^{2} H=H^{\prime \prime}$ & Hessian matrix of $H: \mathbb{R}^{2 n} \rightarrow \mathbb{R}$ \\
\hline$\nabla H=H^{\prime}$ & gradient of $H: \mathbb{R}^{2 n} \rightarrow \mathbb{R}$ \\
\hline$J$ & standard $2 n$ by $2 n$ symplectic form \\
\hline $\mathbf{z}_{\mathrm{cl}}=\left(\mathbf{x}_{\mathrm{cl}}, \mathbf{p}_{\mathrm{cl}}\right)$ & reference orbit \\
\hline $\mathcal{N}_{z_{0}}^{C}$ & Gaussian function on $\mathbb{R}^{2 n}$ with covariance $C$ and mean $z_{0}$ \\
\hline$\psi_{A B}$ & composite pure state \\
\hline$\rho_{A}$ & reduced density matrix $\operatorname{Tr}_{B}|\psi\rangle_{A B}\langle\psi|$ of $\psi_{A B}$ \\
\hline$\Gamma_{A B}$ & inverse covariance matrix of $\psi_{A B}$ \\
\hline$\Gamma_{A}$ & inverse covariance matrix of $\rho_{A}$ \\
\hline$H_{E}$ & entanglement Hamiltonian $\rho_{A}=e^{-H_{E}}$ \\
\hline$W_{\rho}$ & Wigner function of $\rho$ \\
\hline$W_{A B}$ & Wigner function of $\psi_{A B}$ \\
\hline$W_{A}$ & Wigner function $\int_{B} W_{A B}$ of $\rho_{A}$ \\
\hline$\Sigma_{A B}$ & covariance matrix of composite Wigner function $W_{A B}$ \\
\hline & covariance matrix of marginalized Wigner function $W_{A}$ \\
\hline$\rho_{N}(E)=\sum_{n} \delta\left(E-E_{n}\right)$ & spectral density of states \\
\hline$\chi=S(\rho)-\sum_{t} p_{t} S\left(\rho_{t}\right)$ & Holevo's quantity \\
\hline$I(A: B)$ & mutual information between $A$ and $B$ \\
\hline & tripartite information \\
\hline
\end{tabular}


List of symbols 


\section{Contents}

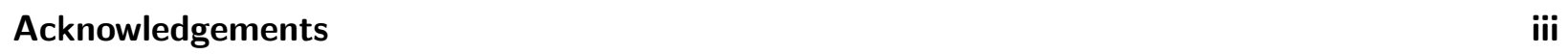

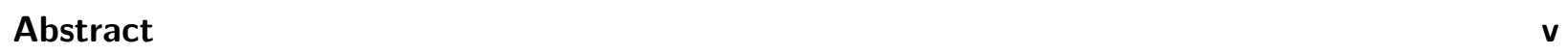

\begin{tabular}{lll}
\hline List of symbols & vii
\end{tabular}

\begin{tabular}{ll}
\hline Contents & ix
\end{tabular}

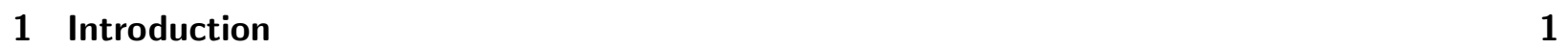

2 Mean field spin model $\mathbf{5}$

$2.1 \quad$ The fully connected transverse field Ising model . . . . . . . . . . . . . . . . . . . . 5

2.2 Effective semiclassical picture . . . . . . . . . . . . . . . . . . . . . . . 8

2.3 General remarks about semiclassics . . . . . . . . . . . . . . . . . . . . . . 10

\begin{tabular}{|l|l}
\hline Publication Ref. [1] & 15
\end{tabular}

3 Entanglement and magnetization variance in the transverse field Ising model 17

3.1 Introduction . . . . . . . . . . . . . . . . . . . 17

3.2 Hierarchy of cumulants $*$. . . . . . . . . . . . . . . . . . . . . . . . . . . . . 18

3.3 Bipartite entanglement . . . . . . . . . . . . . . . . . . . . . . . . . . . . . . 24

3.4 Symplectic squeezing and entanglement in higher dimensions $*$. . . . . . . . . . . 27

3.5 Interlude: bound on entanglement of non-Gaussian states $*$. . . . . . . . . . . . . 32

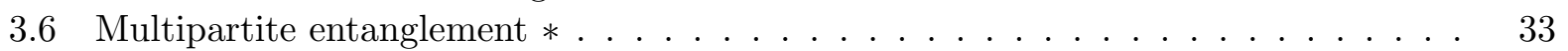

3.7 Inhomogeneous initial states $*$. . . . . . . . . . . . . . . . . . . . . . . 36

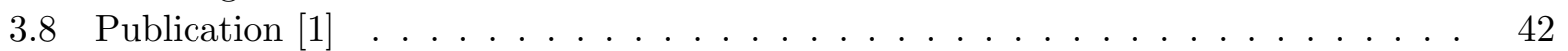

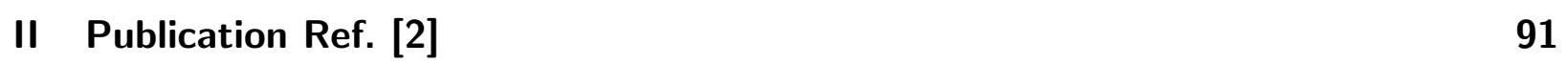

4 Dynamical Phase Transitions 9

4.1 Introduction . . . . . . . . . . . . . . . . . . . . . . . . . . . . . 94

4.2 Phase space heuristics . . . . . . . . . . . . . . . . . . . . . . . . 95

4.3 Semiclassical overlaps . . . . . . . . . . . . . . . . . . . . . . . . . 100

4.4 Discussion . . . . . . . . . . . . . . . . . . . . . . . . 109

4.5 Publication [2] . . . . . . . . . . . . . . . . . . . . . . . . . 111

\begin{tabular}{|lr}
\hline III Entanglement and time & 119
\end{tabular}

\begin{tabular}{|ll|}
\hline History state entanglement & 121
\end{tabular}

5.1 Extended Hilbert space and history states . . . . . . . . . . . . . . . . . . . . . . . 122

5.2 History state entanglement entropy . . . . . . . . . . . . . . . . . . . . . . . . . . . . 124 
Contents

5.3 Interpretation of the history entanglement $\ldots \ldots \ldots \ldots \ldots \ldots$. . . . . . . . . 126

5.4 Wigner random matrix . . . . . . . . . . . . . . . . . . . . . . 133

5.5 Hardcore boson lattice model $\ldots \ldots \ldots \ldots$. . . . . . . . . . . . . . . . 140

$\begin{array}{llr}6 & \text { Conclusion and outlook } & 147\end{array}$

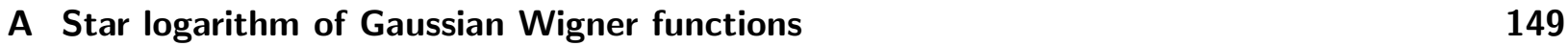

\begin{tabular}{|ll}
\hline B Dicke state entanglement entropy & 153
\end{tabular}

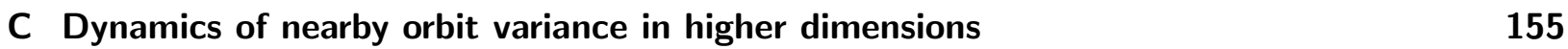

\begin{tabular}{ll}
\hline Bibliography & 157
\end{tabular} 


\section{Introduction}

(Quantum) Information. From the epistemological point of view of digital physics [3, 4], the fundamental entity that governs all physical processes and phenomena, is information and information processing, i.e., computation. An early hint that information has physical significance, is Maxwell's famous demon [5], and its derivates due to Smoluchowski [6], Szilard [7] and Feynman [8]. Today, information has a precise quantitative meaning in thermodynamics. Heat dissipation as a consequence of information erasure [9], and its use as a source for engines [10] is well established.

In quantum physics, information and information processing plays a similarly important role [11]. However, compared to classical information, quantum information is notably different. Foremost, in contrast to classical information, conditional quantum information can be negative. As a consequence, "the best possible knowledge of a whole does not necessarily include the best possible knowledge of all its parts, even though they may be entirely separate" [12]. This underlies the phenomenon of quantum entanglement, which has no classical analog.

Since the years of its first investigations in 1935 [12,13, entanglement has developed into an indispensable tool. The applications of entanglement range from black hole thermodynamics [1416], the fundamental structure of space-time [17], holographic dualities [18, 19], conformal field theories [20 23], quantum information theory [24] and quantum computation [25], to condensed matter physics [26].

In the field of condensed matter physics, entanglement is used as an order-parameter-independent quantity to identify quantum phase transitions [27,28]. The effects of entanglement can enhance measurement accuracy in quantum metrology [29,30], and protect non-locally stored information against decoherence in quantum computation [31]. Entanglement is a bottleneck for many numerical methods, such as the density matrix renormalization group [32 34$]$ and in the matrix product states formulation [35, 36], as they rely on the fact that the entanglement remains low in order to effectively avoid the complexity of the exponential Hilbert space dimension. Due to finite computational resources, this is particularly limiting in out-of-equilibrium situations, where entanglement of typical states approaches a volume law 23, 37, contrary to ground states that typically obey an area law. Whether entanglement scales with the volume or the area of the bipartition, in both cases the induced complexity grows with dimension. From this perspective, the understanding of the time evolution of entanglement is crucial.

Quantum simulators. One of the main long-term technological goals at the intersection of nonequilibrium quantum many body physics and quantum information theory is the construction of a large scale quantum computer. Several recent theoretical advancements, such as topologically protected states [38], and the application of machine learning algorithms to quantum stabilization [31, 39], are exploited to achieve long-time quantum coherence. Even though some breakthroughs have been made [40], the dream of a large scale quantum computer is not completely realized, yet.

Because "[the] thought of every age is reflected in its technique" [41], the theoretical approach to quantum computing is tightly entangled with experimental progress. In addition to quantum computers, which may also be called digital quantum simulators, analog quantum simulators have also gained attention over the past years. The increasing control of experimental setups have lead to the realization of Manin's and Feynman's [42 44] vision, and induced the following paradigm shift. On the one hand, theoretical models are devised to explain and predict experimental observations. 


\section{Introduction}

These models are simple enough to be mathematically or numerically accessible, and yet, detailed enough to capture the essential (often universal) features of the matter of interest. The modeling of solid state materials by lattice Hamiltonians and effective degrees of freedom is along those lines. Another prime example is the use of random Hamiltonians to describe the universal features of the spectra of heavy nuclei [45]. On the other hand, analog quantum simulators in some sense reverse this process of theoretical modeling. Ultra cold quantum gases on optical lattices and traps are prime examples for analog quantum simulators [46 49]. By controlling the particles of the gas, it is possible to simulate a given theoretical quantum Hamiltonian and its induced Schrödinger dynamics in the lab.

Quantum many body physics out of equilibrium. Questions regarding non-equilibrium quantum physics fall into two main categories. The first category concerns what it means that a quantum system thermalizes [50], whether or not specific systems thermalize, and how they do so [51], e.g. on which time scales [52. In other words, the problem of thermalization is about the microscopic justification of (quantum) thermodynamics. Related to this is the question what good notions of quantum integrability are, and whether and how integrable systems evolve differently from non-integrable systems [53,54].

The second category is about the description of the dynamics and classification of non-thermal (steady) states of matter. In recent years, novel non-equilibrium states that rely on both, the many particle nature, as well as the quantum nature, have been found. Sometimes, a single or few body quantum phenomenon, such as Anderson localization, and quantum scars in semiclassical wave functions, have a rich many body analog, such as quantum many body localization 55, 56 and quantum many body scars [57, 58]. Quantum many body localization is an example for a non-thermal state of matter and is characterized by slow logarithmic increase of entanglement. Moreover, recently found new types of dynamical behavior, such as dynamical phase transition: 1 [59, 60], and time crystals [61], are structurally analogous to equilibrium physics.

The focus of this thesis is on the second category of questions. In particular, the non-thermalizing dynamics of a permutation invariant spin model is studied. More details are given below.

Fully-connected models. In this treatise, the fully connected transverse field Ising model (FC-TFIM) is investigated out of equilibrium. This model, in which $N$ spins are coupled in a permutation invariant way, is also known as the Lipkin-Meshkov-Glick (LMG) model [62], and can also be formulated as a two component Bose-Einstein condensate (BEC) 63 65]. The non-equilibrium situation is achieved by a sudden quantum quench in the transverse magnetic field.

The study of this particular model is motivated by three main reasons. First, it has a mathematically amenable thermodynamic limit as $N \rightarrow \infty$. More precisely, the thermodynamic limit is identical to the mean field limit. Corrections to the mean field limit become important for large, but finite $N$. These corrections are non-trivial and can be obtained in a controlled $1 / N$ expansion. This expansion is non-perturbative in the couplings of the model, and therefore, provides a complementary approach to perturbative treatments of many body problems. By studying the corrections to the mean field limit, the FC-TFIM provides an example to benchmark the validity of mean field approximations out of equilibrium.

Second, the FC-TFIM can be viewed as the infinite range limit of a one-dimensional spin model with long-range couplings. Those long-range models are of experimental [46 48 ] and theoretical interest. One question is how the spread of correlations $[66-69]$ and entanglement $[70-75]$ in longrange models is different from their short range counter parts [22, 23, 76, 77]. Many results on entanglement dynamics in long range models are numerical $70,78,79]$ and semi-analytical $[72,80]$.

\footnotetext{
${ }^{1}$ Two different types of dynamical phase transition are discussed in Sec. 4 of this thesis.
} 
The infinite coupling limit provides an accessible model to address the entanglement dynamics analytically.

Third, fully connected models may also be viewed as effectively infinite dimensional lattice models, in the sense that their coordination number diverges. As such, they provide the opposite limit to low dimensional, in particular one-dimensional, systems. In contrast to notoriously difficult two and three-dimensional transverse field Ising model, the infinite-dimensional limit is accessible.

Outline. This thesis is structured as follows. In chapter 2 a short introduction to the FC-TFIM is given. The permutation invariance and its consequences are emphasized. In particular, the mapping onto an effective one-dimensional model is explained. Under this mapping the thermodynamic limit of the spin model translates to the classical limit of the effective model. Therefore, some remarks on semiclassical theory are made, and a few results, which will be used in later chapters, are collected. The material of this chapter is not new, and rather written in a monograph style.

The subsequent material is structured in three parts, each part is contained in one of the chapters 3. 4, and 5. The chapters 3 and 4 are devoted to the author's publications [1] and [2], respectively, while chapter 5 contains unpublished results.

In [1], see Sec. 3.8, the dynamics of the mean and variance of the magnetization after a quantum quench is obtained analytically to leading order as $N \rightarrow \infty$. Based on these observations the dynamical phase diagram is explained, and the time scale of validity of the mean field approximation is discussed. As a consequence of spin-squeezing the magnetization variance is tightly connected to entanglement. The leading contribution to the entanglement Hamiltonian is computed in the large $N$ limit. The entanglement Hamiltonian is a harmonic oscillator, and the quantitative relation between spin squeezing and Rényi entanglement entropies is derived.

In addition to these results, chapter 3 discusses three extensions not contained in [1]. First, in Sec. 3.2, the dynamics of higher cumulants beyond the variance is investigated. The hierarchical coupling of the cumulants' equations of motion is derived and discussed. Second, in Sec. 3.4, the calculation of the entanglement Hamiltonian and Rényi entanglement entropies of Gaussian states is generalized to higher state space dimensions. A semiclassical interpretation in terms of the symplectic capacity and the symplectic non-squeezing theorem is given. This result is used in Sec. 3.5 to bound the von Neumann entanglement entropy of non-Gaussian states from above. The result of Sec. 3.4 also facilitates a short discussion of quadripartite entanglement in the FC-TFIM, cf. Sec. 3.6. Third, the dynamics of inhomogeneous initial states in the FC-TFIM is briefly sketched in Sec. 3.7. It is speculated on the consequences for the dynamics of spin squeezing and entanglement.

Chapter 4 is concerned with dynamical phase transition (DPT) in the FC-TFIM. After a brief review of the literature in Sec. 4.1, two independent approaches to DPT in the FC-TFIM are presented in Secs. 4.2 and 4.3 . Both sections suggest the existence of DPT in the spin model. The first approach in 4.2 is rather heuristic. The second approach in 4.3 gives a more microscopic account of the occurrence of DPT. In particular, the critical times at which DPT occur, and the decay of the Loschmidt rate function are related to spectral properties of the FC-TFIM. Limitations are critically discussed.

A copy of the publication [2] is attached in Sec. 4.5. This publication discusses a connection between two different types of DPT in the FC-TFIM, and is mainly based on numerical observations.

Chapter 5 is independent of the other chapters and self-contained. It discusses bipartite entanglement entropy in Feynman history states as a quantum information theoretic characterization of 


\section{Introduction}

the dynamics in closed quantum systems. Common to both, the DPT investigated in chapter 4 and the history entanglement in chapter 5 , is the crucial role of the spectral density of the Hamiltonian driving the dynamics.

After reviewing and introducing the notion of extended Hilbert spaces, history states and history entanglement in Secs. 5.1 and 5.2 , two information theoretic interpretations of the history entanglement are given in Sec. 5.3. One is based on the entanglement entropy of measurement, the other relies on Holevo's bound. A connection to quantum clocks and quantum speed limits is emphasized. The examples of a random matrix ensemble, cf. Sec. 5.4, and a hardcore boson model in the integrable and non-integrable regime, cf. Sec. 5.5, show how the history entanglement discriminates between integrable and non-integrable dynamics. The analytical predictions are confirmed by numerical data. 


\section{Mean field spin model}

The following chapters 3 and 4 discuss non-equilibrium quantum dynamics after a quench in the transverse field Ising model of fully connected spins. This chapter gives a brief introduction into the infinite range Ising model and semiclassical analysis. This is textbook knowledge, and does not contain any new results.

After stating the fully connected Ising Hamiltonian and discussing the consequences of its permutation invariance in Sec. 2.1, we review the mapping onto an effective semiclassical model in the large $N$ limit in Sec. 2.2, Thereafter, in Sec. 2.3, we make a brief digression into semiclassical analysis.

\subsection{The fully connected transverse field Ising model}

The Hamiltonian of the transverse field Ising model of $N$ fully connected spins reads

$$
H=-\frac{1}{2 N} \sum_{i, j=1}^{N} s_{i}^{z} s_{j}^{z}-\Gamma \sum_{i=1}^{N} s_{i}^{x},
$$

where $s_{i}^{x, y, z}=\sigma_{i}^{x, y, z} / 2$ are spin- $1 / 2$ operators of the $i$ th elementary spin in terms of the Pauli matrices $\sigma^{x, y, z}$, and $\Gamma$ is the homogeneous transverse magnetic field. The ferromagnetic coupling between the spins is all-to-all, and its uniform strength is scaled by a factor of $1 / N$ such that both sums in 2.1) scale extensively as $\mathcal{O}(N)$.

The model (2.1) is also known as the Lipkin Meshkov Glick model 62]. It has a quantum critical point at $\Gamma_{c}=1 / 2$, which separates the ferromagnetic (ordered) phase $\Gamma<\Gamma_{c}$ from the paramagnetic (disordered) phase $\Gamma>\Gamma_{c} 28,81$.

The Hamiltonian (2.1) is defined on the $2^{N}$-dimensional Hilbert space $\mathcal{H}_{N}=\otimes^{N} \mathbb{C}^{2}$ of $N$ elementary $1 / 2$ spins. The subspace of invariant states w.r.t. permutations of the $N$ spins is denoted by $\mathcal{D}_{N}$, and referred to as the symmetric Dicke space. As $H$ is invariant under permutations of the spins, the Dicke subspace is invariant under $H$ and under the family of unitary time evolution $U=e^{-i H t}$.

In Sec. 2.2 we will encounter another Hilbert space, the Hilbert space of square integrable functions on the unit interval $\mathcal{H}_{\text {eff }}:=L^{2}(0,1)$. In the limit of $N \rightarrow \infty$, states in $\mathcal{D}_{N}$ are approximated by functions in $\mathcal{H}_{\text {eff. }}$ The three Hilbert spaces, $\mathcal{H}_{N}, \mathcal{D}_{N}$, and $\mathcal{H}_{\text {eff }}$ occur frequently in this treatise. Pictorially, their relation is summarized as

$$
\mathcal{H}_{N} \supset \mathcal{D}_{N} \stackrel{N \rightarrow \infty}{\longrightarrow} \mathcal{H}_{\text {eff }}
$$

In the following subsection, we discuss the symmetric Dicke space and its relation to the full Hilbert space of $N$ spins in more detail. Thereafter, in Sec. 2.2 a mapping of the symmetric spin model onto an effective model with Hilbert space $\mathcal{H}_{\text {eff }}$ is explained. This effective description has a semiclassical limit for $N \rightarrow \infty$. 


\subsubsection{Permutation invariance and Dicke subspace}

We spend some time on the theory of permutation invariant systems. First, we review some general consequences of permutation invariant Hamiltonians on the structure of the Hilbert space by following [82]. Although, we will mostly confine to the totally symmetric subspace, we sketch the theory in more generality. Second, we apply the general findings to a fully symmetric spin Hamiltonian of $N$ spin-1/2 particles.

Generic permutation invariant Hamiltonian of $N$ particles. The following discussion can be found in chapter IX. of 82 . Let $H$ be a generic $N$ particle Hamiltonian ${ }^{1}$ that commutes with all particle permutation operators $P$, i.e. $[H, P]=0$. Moreover, any permutation operator is unitary, $P^{\dagger}=P$, and can be viewed as a conserved (complex-valued) quantity. There are $N$ ! conserved quantities resulting from permutation invariance in this way. However, as two permutation operators $P_{1}$ and $P_{2}$ do not commute in general, one cannot give a value to all these conserved quantities simultaneously. In other words, the permutation operators are complementary, and there is no common eigenbasis of all $P$ and $H$. The best one can do is to give a maximal set of pairwise commuting conserved quantities $\chi_{1}, \cdots \chi_{m}$, i.e. $\left[\chi_{i}, \chi_{j}\right]=0$ and $\left[\chi_{i}, H\right]=0$. Every $\chi_{i}$ must be a linear combination of the permutation operators, because any polynomial of permutation operators can be written as a linear combination of permutation operators. It turns out that the choice of $\chi_{i}=\chi\left(P_{i}\right)=(N !)^{-1} \sum_{P} P P_{i} P^{\dagger}$, where the summation is over all permutations, fulfills the requirements. Evidently, $\chi_{i}$ commutes with all permutations. Two similar ${ }^{2}$ permutations $P$ and $Q$ give identical $\chi(P)=\chi(Q)$. On the contrary, any two permutations $P$ and $Q$ that are not similar yield different operators, $\chi(P) \neq \chi(Q)$. Hence, to each equivalence class of similar permutations one associates a conserved quantity $\chi_{i}$. As $\chi_{i}$ is the average over all permutations in the respective equivalence class, and because any permutation $P$ is similar to its inverse $P^{\dagger}$, $\chi_{i}$ is Hermitian, i.e. a real-valued conserved quantity. The number of equivalence classes modulo similarity is $m$, the number of ways that $N$ can be decomposes into a sum of integers. A trivial conserved quantity is given by the identity, $\chi_{1}=1$. We denote the collection of all $m$ operators $\left(\chi_{1}, \cdots \chi_{m}\right)$ by the bold face letter $\chi$. The eigenvalues of $\chi$ are not independent, since they have to fulfill certain relations among them. It turns out that there are $m$ different valid solutions (each solution is related to a character of the group of permutations). One obvious solution is $\chi_{1}=1, \cdots \chi_{m}=1$, corresponding to permutation invariant states. Another solution is $\chi_{i}= \pm 1$, depending on the parity of the equivalence class, corresponding to antisymmetric states. These two irreducible representations of the permutation group, the totally symmetric and the totally antisymmetric, are the most prominent ones, as they are the mathematical basis of the theory bosons and fermions, respectively. We can now construct common eigenstates of $H$ and $\chi$, and denote the orthonormal eigenbasis by $|E, \chi\rangle$. The permutation invariant energy eigenstates are $\left|E,\left\{\chi_{i}=1\right\}\right\rangle$. These states are special and they obey $P\left|E,\left\{\chi_{i}=1\right\}\right\rangle=\left|E,\left\{\chi_{i}=1\right\}\right\rangle$ for any permutation $P$. In general, acting with a permutation on $|E, \chi\rangle$ changes the state, but it remains an eigenstate of $H$ and $\chi$ with the same eigenvalues. In other words, $|E, \chi\rangle$ are usually highly degenerate. (The fact that many energy eigenstates are degenerate already follows from the fact that $H$ has many non-commuting conserved quantities.) The degeneracy $\left.\right|^{3}$ of $|E, \chi\rangle$ is a function

\footnotetext{
${ }^{1}$ For the sake of concreteness, one may think of $H$ as the Hamiltonian in 2.1, but the discussion applies to all $N$ particle Hamiltonians of any particle type.

${ }^{2}$ Two permutations $P_{1}$ and $P_{2}$ are similar if there exists another permutation $P$ such that $P_{1}=P P_{2} P^{-1}$. In other words, two permutations $P_{1}$ and $P_{2}$ are similar if they are identical modulo relabeling of the labels by a permutation $P$.

${ }^{3}$ Here, we only mean the degeneracy as a consequence of the indistinguishability of the particles. Of course, there may be additional degeneracies, unrelated to the permutation invariance.
} 
$n(\chi)$ of $\chi$ alone. It is impossible to distinguish these degenerate eigenstates by physically meaningful, i.e. permutation invariant, operators. Only eigenstates of $\chi$ with different eigenvalues can be distinguished by physically meaningful observables. Examples of such meaningful observables are the Hermitian operators $\boldsymbol{\chi}$.

Permutation invariant Hamiltonian of $N$ spin- $1 / 2$ particles. Now, we turn to the special situation of a permutation invariant Hamiltonian of $N$ spin one half particles, i.e. when the local Hilbert space of a single particle is two dimensional. Prior to discussing the concrete spin Hamiltonian (2.1), we make some general remarks about the structure of the Hilbert space of a system of $N$ identical spin one-half particles. A system of $N$ elementary spins is built up by starting with a single spin- $1 / 2$ and successively adding elementary spins one by one until the $N$-th spin is added. Adding two spin one half spins produces an antisymmetric singlet $(j=0)$ state and three symmetric triplet states $(j=1)$. The procedure of subsequently adding more spins is schematically sketched in Fig. 2.1. From one row to the next a spin one half is added. The numbers in every

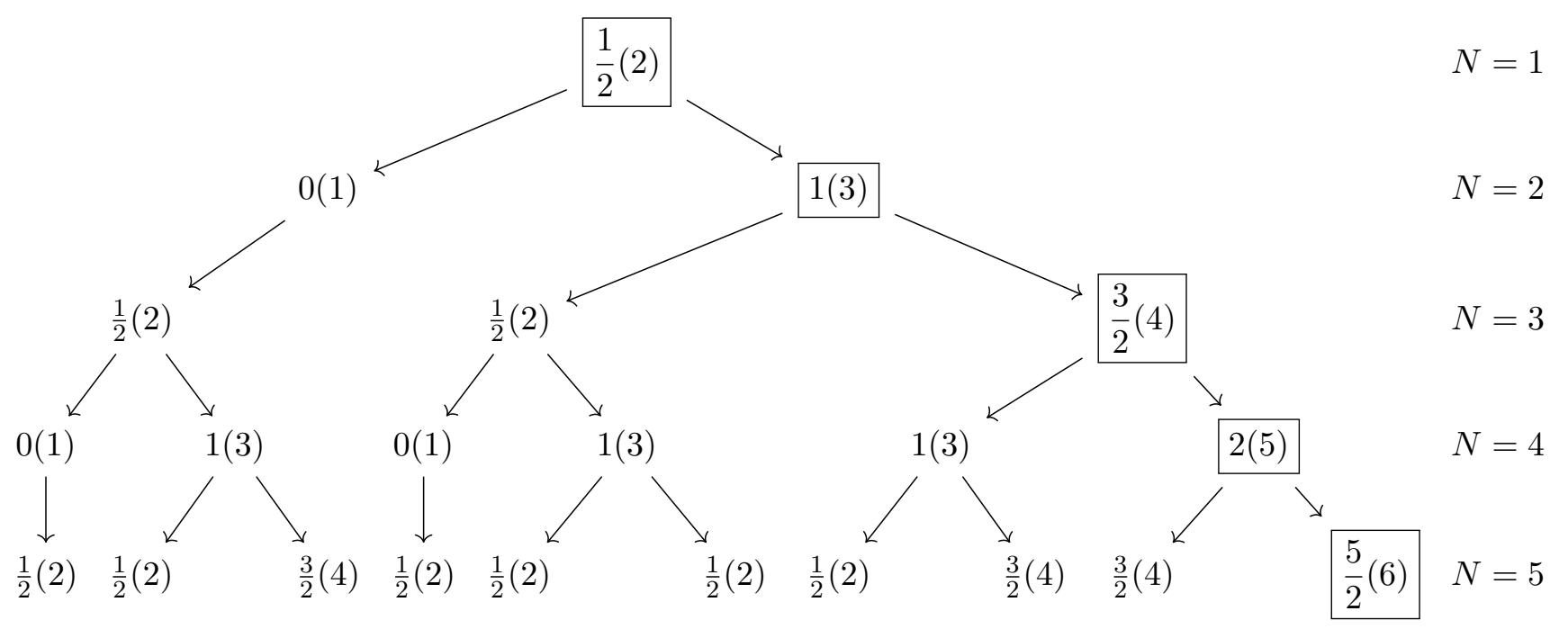

Figure 2.1: Successive buildup of the Hilbert space of $N$ spin-1/2 particles, starting with a single spin (top row), and adding one additional spin according to the spin coupling rules by going to the next row below. Each cell represents subspace of the Hilbert space with fixed number of particles (given by the row index $N$ in the last column), and a fixed total spin $j$ given by the first number in each cell. The number in brackets is $(2 j+1)$, the dimension of the subspace. All of these subspaces are mutually orthogonal. The total dimension of all subspaces in the $N$-th row sums to $2^{N}$, the dimension of the Hilbert space of $N$ spin-1/2 particles. The last cell in each row (boxed) denotes the totally symmetric, permutation invariant, Dicke subspace of $N$ spins.

cell indicates the spin- $j$ representation, the number in brackets is the dimension $(2 j+1)$ of this representation. The sum of all dimensions in the $N$ th row add up to $2^{N}$, i.e. the Hilbert space dimension of $N$ elementary spins. The arrows indicate how the irreducible spin- $j$ representations splits into the two irreducible spin $|j-1 / 2|$ and $(j+1 / 2)$ representations upon adding a new spin. Thereby, the dimension doubles from $(2 j+1)$ to $[2(j-1 / 2)+1]+[2(j+1 / 2)+1]=2(2 j+1)$. The representation with the largest spin in each row (marked with a box) is totally symmetric, 


\section{Mean field spin model}

i.e. states of this representation are eigenvalue one eigenstates of $\chi_{1}, \cdots \chi_{m}$ acting on the $N$ elementary spins. Those totally symmetric states span the $(N+1)$ dimensional Dicke space. In other words, the Dicke space is the spin- $N / 2$ irreducible representation obtained by adding $N$ elementary spin-1/2's. In general, the representations of the remaining cells in the above diagram are not spanned by $\chi$ eigenstates. However, in the direct product space of representations of the same spin $j$, one can choose an eigenbasis of the $\chi_{1}, \cdots \chi_{m}$ operators. This is because the operators $\chi$ commute with the total spin operator $\mathbf{S}=\sum_{j}^{N} \mathbf{S}_{j}$, and hence, eigenstates of $\chi$ must lie in an eigenvalue $j(j+1)$ eigenspace of $\mathbf{S}^{2}$. We do not proceed further with the discussion of the non-symmetric $\chi$ eigenspaces, other than noting that these eigenspaces need to be taken into account when constructing thermal density matrices of permutation invariant spin Hamiltonians on $\mathcal{H}_{N}=\left(\mathbb{C}^{2}\right)^{\otimes N}$.

It is interesting that the spin- $N / 2$ representation obtained by adding $N$ elementary spin- $1 / 2$ 's is invariant under permutations of the spins. We confirm this fact by a direct computation. To this end, let

$$
\left|N_{+}\right\rangle=\left(\begin{array}{c}
N \\
N_{+}
\end{array}\right)^{1 / 2} P|\uparrow \cdots \uparrow \downarrow \cdots \downarrow\rangle
$$

be the normalized permutation invariant state of $N$ elementary spins with $N_{+}$up spins, where $P=\frac{1}{N !} \sum_{p \in \mathcal{S}_{N}} p$ is the orthogonal projection operator onto the permutation invariant subspace. There are $(N+1)$ independent states, parametrized by $N_{+} \in\{0, \cdots N\}$. We show that these states are $\frac{N}{2}\left(\frac{N}{2}+1\right)$ eigenvalue eigenstates of $\mathbf{S}^{2}$. The off-diagonal terms in the double sum $\mathbf{S}^{2}=\sum_{i, j=1}^{N} \mathbf{S}_{i} \cdot \mathbf{S}_{j}$ act on direct product states $\left|s_{i}, s_{j}\right\rangle$, with $s_{i}, s_{j} \in\{\uparrow, \downarrow\}$, as

$$
\mathbf{S}_{i} \cdot \mathbf{S}_{j}\left|s_{i}, s_{j}\right\rangle=\frac{1}{4} \begin{cases}2\left|\bar{s}_{i}, \bar{s}_{j}\right\rangle-\left|s_{i}, s_{j}\right\rangle, & s_{i} \neq s_{j} \\ \left|s_{i}, s_{j}\right\rangle, & s_{i}=s_{j},\end{cases}
$$

where $\bar{s}$ denotes the spin obtained by flipping $s$, i.e. $\bar{\uparrow}=\downarrow$ and $\bar{\downarrow}=\uparrow$. In both cases, for a pair of parallel and antiparallel spins, the number of up and down spins is not changed under the action of $\mathbf{S}_{i} \cdot \mathbf{S}_{j}$. Using this result and the fact that $\left|s_{i}\right\rangle$ is an $\frac{1}{2}\left(\frac{1}{2}+1\right)$ eigenstate of $\mathbf{S}_{i}^{2}$ in

$$
\begin{aligned}
\mathbf{S}^{2}\left|N_{+}\right\rangle & =\mathbf{S}^{2}\left(\begin{array}{c}
N \\
N_{+}
\end{array}\right)^{1 / 2} P|\uparrow \cdots \uparrow \downarrow \cdots \downarrow\rangle \\
& =\left(\begin{array}{c}
N \\
N_{+}
\end{array}\right)^{1 / 2} P\left[N \frac{1}{2}\left(\frac{1}{2}+1\right)+\sum_{i \neq j} \mathbf{S}_{i} \cdot \mathbf{S}_{j}\right]|\uparrow \cdots \uparrow \downarrow \cdots \downarrow\rangle \\
& =\left[N \frac{1}{2}\left(\frac{1}{2}+1\right)+N(N-1) \frac{1}{4}\right]\left(\begin{array}{c}
N \\
N_{+}
\end{array}\right)^{1 / 2} P|\uparrow \cdots \uparrow \downarrow \cdots \downarrow\rangle \\
& =\frac{N}{2}\left(\frac{N}{2}+1\right)\left|N_{+}\right\rangle
\end{aligned}
$$

shows that the symmetric Dicke states are indeed states of a spin- $N / 2$ representation.

\subsection{Effective semiclassical picture}

We are interested in two things. First, expectation values $\langle\Psi|O| \Psi\rangle$ of permutation invariant operators $O$ in a typical state $|\Psi\rangle \in \mathcal{D}_{N}$. And second, the time evolution of $|\Psi\rangle$ w.r.t. $H$, that is $\left|\Psi_{t}\right\rangle=e^{-i H t}|\Psi\rangle$. In general, $\langle O(t)\rangle$ is not a simple explicit expression. However, in the limit $N \rightarrow \infty$, and under certain conditions, the leading contribution of $\langle O(t)\rangle$ evolves according to an 
effective quantum system in the semiclassical limit.

More precisely, for large $N$ there is an approximate mapping of the spin model $(2.1)$ on $\mathcal{D}_{N}$ to a quantum system of a fictitious particle on the interval $[0,1]$ with effective Planck constant $\hbar_{\mathrm{eff}}=1 / N$ and Hamiltonian 81

$$
H_{\mathrm{eff}}\left(n_{+}, p\right)=-\frac{1}{2}\left(n_{+}-1 / 2\right)^{2}-\Gamma \sqrt{n_{+}-n_{+}^{2}} \cos (p),
$$

where $p=-i \hbar_{\mathrm{eff}} \partial_{n_{+}}$. This effective Hamiltonian is defined on the Hilbert space $\mathcal{H}_{\text {eff }}=L^{2}(0,1)$ of square integrable functions on the unit interval. The position of the fictitious particle is given by the fraction of up spins $n_{+}=N_{+} / N$ in the spin model.

In the limit $N \rightarrow \infty$ the mapping becomes exact, and the evolution of $\langle O\rangle$ follows from the classical equations of motion of $H_{\text {eff }}$. This is the mean field limit. If $N$ is sufficiently large, but still finite, then, quantum fluctuations around the classical limit can be taken into account by a systematic expansion in $1 / N$. One quantitative measure for these quantum fluctuation effects is the variance $\operatorname{var}(O)=\left\langle O^{2}\right\rangle-\langle O\rangle^{2}$. We will see in Sec. 3.2 how to systematically approximate the variance $\operatorname{var}(O)$ and higher moments. The fact that quantum fluctuations are included on top of the classical limit, is referred to as semiclassics.

The derivation of the effective Hamiltonian (2.3) is described in more detail in [81] and in appendix A of [1]. A generalization of the effective Hamiltonian to the situation of inhomogeneous (i.e. not fully permutation invariant) states is described in Sec. 3.7 .

Essentially, the Hamiltonian $H_{\text {eff }}$ follows from expanding the spin Hamiltonian $H$ of Eq. (2.1) in the Dicke basis states $\left|N_{+}\right\rangle$, cf. Eq. (2.2). The key point of the effective description is the right choice of coordinates $n_{+}=N_{+} / N$. In order to have a well defined limit $\hbar_{\text {eff }}=1 / N \rightarrow 0$ the coordinates of the effective configuration space must scale intensively, i.e. independent of $N$, in the large system size limit, cf. table 2.1. The fraction of up spins, $n_{+}=N_{+} / N$ is a suitable intensive coordinate. The percental quantity $\left(n_{+}+1 / 2\right)$ is the eigenvalue of the intensive collective spin operator $s_{z}=\frac{1}{N} \sum_{i=1}^{N} \sigma_{i}^{z} / 2$ with eigenstate $\left|N_{+}\right\rangle$. The commutation relations of the intensive collective spin operators $\mathbf{s}=\left(s_{x}, s_{y}, s_{z}\right)$ is given by the $\mathrm{SU}(2)$ algebra decorated with the scaling parameter $\hbar_{\mathrm{eff}}=1 / N$, i.e. $\left[s_{x}, s_{y}\right]=\hbar_{\mathrm{eff}} s_{z}$, and similarly for cyclic permutations of the indices. The fact that the commutator vanishes for $N \rightarrow \infty$ is another instance of why the intensive spin operators facilitate a semiclassical description. The change of coordinates from $N_{+}$to $n_{+}=N_{+} / N$ can be interpreted as a scale separation [83], where $\hbar_{\mathrm{eff}}=1 / N$ plays the role of the scaling parameter.

Remark (on the order of limits, $N \rightarrow \infty$ and $t \rightarrow \infty$ ). In general, the mean field limit $N \rightarrow \infty$ and the large time limit $t \rightarrow \infty$ do not commute. This has far-reaching consequences and is the reason for the rich and complex structures in the field of quantum chaos (quantum chaology) [84. For example, the double limit of $N \rightarrow \infty$ and $t \rightarrow \infty$ was investigated in [85] for the spin model (2.1) with $\Gamma=0$. It was shown that the trace of the evolution operator $e^{-i t H}$ can lead to complicated fractal structures in the complex plane (even in the supposedly simple case of $\Gamma=0$ ). We are not concerned with these complications, though. Instead, we investigate the asymptotic behavior for a fixed large $N$ and early times. Because of the aforementioned, we cannot expect the asymptotic results to hold for arbitrarily large time scales. Ref. [1] discusses the time scale of the breakdown of the mean field description as a function of $N$.

Remark (on different semiclassical approaches). We follow the approach [81] of expanding $H$ in the Dicke states $\left|N_{+}\right\rangle$to obtain the effective description $H_{\text {eff. }}$ This is not the only semiclassical technique in the context of spin models. Another approach is an expansion in spin coherent

\footnotetext{
${ }^{4}$ The approximation involved in the mapping from $\left(\mathcal{D}_{N}, H\right)$ to $\left(\mathcal{H}_{\mathrm{eff}}, H_{\mathrm{eff}}\right)$ consists in neglecting terms of order $\mathcal{O}(1 / N)$ in $H_{\text {eff. }}$ More details are given in appendix A of 1 .
} 
states 86. One reason, why we use an expansion into Dicke states is, that a bipartition of the elementary spins is very natural for the Dicke states, but less obvious for spin coherent states. We use this bipartition in the discussion of entanglement in Sec. 3.3 .

Summary. The relations between the fully connected spin model (2.1) on $\mathcal{D}_{N}$ and its effective description $H_{\text {eff }}$ on $\mathcal{H}_{\text {eff }}$ are summarized in table 2.1 .

\begin{tabular}{|c|c|c|}
\hline & collective spin model & effective description \\
\hline limit & $\begin{array}{r}N \rightarrow \infty, \text { thermodynamic limit } \\
\text { mean field limit }\end{array}$ & $\hbar_{\mathrm{eff}}=1 / N \rightarrow 0$, classical limit \\
\hline corrections & $\begin{array}{r}\text { finite size effects, corrections to } \\
\text { mean field }\end{array}$ & quantum fluctuations \\
\hline configuration & $N_{+} \in \mathbb{Z}_{N+1}$ (extensive) & $n_{+}=N_{+} / N \in[0,1]$ (intensive) \\
\hline Hilbert space & $\mathcal{D}_{N} \cong l^{2}\left(\mathbb{Z}_{N+1}\right)($ Dicke space $)$ & $\mathcal{H}_{\text {eff }}=L^{2}(0,1)$ \\
\hline basis & $\left\{\left|N_{+}\right\rangle\right\},\left\langle N_{+} \mid M_{+}\right\rangle=\delta_{N_{+}, M_{+}}$ & $\left\{\left|n_{+}\right\rangle\right\},\left\langle n_{+} \mid m_{+}\right\rangle=\delta\left(n_{+}-m_{+}\right)$ \\
\hline states & $\Psi\left(N_{+}\right)=\left\langle N_{+} \mid \Psi\right\rangle$ & $\psi\left(n_{+}\right) \approx \sqrt{N} \Psi\left(\left\lfloor n_{+} N\right\rfloor\right)$ \\
\hline normalization & $\|\Psi\|_{\mathcal{D}_{N}}=\sum_{N_{+}=0}^{N}\left|\Psi\left(N_{+}\right)\right|^{2}=1$ & $\|\psi\|_{\mathcal{H}_{\mathrm{eff}}}=\int_{0}^{1} \psi\left(n_{+}\right)=1$ \\
\hline collective & $S_{z}=\sum_{i=1}^{N} \sigma_{i}^{z} / 2$ (extensive) & $\left(n_{+}-1 / 2\right)$ (multiplication) \\
\hline spin & $S_{x}=\sum_{i=1}^{N} \sigma_{i}^{x} / 2$ (extensive) & $\cos (p), p=-i \partial_{n_{+}} / N$ \\
\hline Hamiltonian & $\begin{array}{l}H=-\frac{1}{2 N} S_{z}^{2}-\Gamma S_{x} \\
\text { (extensive), Eq. 2.1) }\end{array}$ & $H_{\text {eff }}$, (intensive), Eq. 2.3 \\
\hline \multirow[t]{2}{*}{ eigenenergies } & $H\left|E_{n}\right\rangle=E_{n}\left|E_{n}\right\rangle$ & $H_{\mathrm{eff}} \psi_{n}=e_{n} \psi_{n}$ \\
\hline & $E_{n}$ (extensive) & $e_{n} \approx E_{n} / N$ (intensive) \\
\hline eigenstates & $\Psi_{n}\left(N_{+}\right)=\left\langle N_{+} \mid E_{n}\right\rangle$ & $\psi_{n}\left(n_{+}\right) \approx \sqrt{N} \Psi_{n}\left(\left\lfloor n_{+} N\right\rfloor\right)$ \\
\hline
\end{tabular}

Table 2.1: Summary of various relations between the fully connected spin model 2.1$)$ on $\mathcal{D}_{N}$ and its effective description 2.3 on $\mathcal{H}_{\text {eff. }}$

The upshot is that the thermodynamic limit in the fully connected Ising model (2.1) translates to the classical limit in the effective model (2.3). We will make intensive use of semiclassical analysis in the effective model to study the quantum dynamics in the mean field spin model for asymptotically large $N$. In particular, we apply the ideas of the truncated Wigner approximation 87,88 and the nearby orbit approximation 89 91. Schematically,

\begin{tabular}{|c|c|c|c|c|c|c|}
\hline $\begin{array}{l}\text { thermodynamic limit } \\
\qquad N \rightarrow \infty \\
\text { in collective spin model }\end{array}$ & $\longrightarrow$ & $\begin{array}{c}\text { semiclassical limit } \\
\hbar_{\mathrm{eff}}=N^{-1} \rightarrow 0 \\
\text { in effective description }\end{array}$ & $\longrightarrow$ & $\begin{array}{c}\text { truncated Wigner } \\
\text { approximation (TWA) }\end{array}$ & $\longrightarrow$ & $\begin{array}{c}\text { nearby orbit } \\
\text { approximation }\end{array}$ \\
\hline
\end{tabular}

In the following section we collect some general semiclassical results for later reference.

\subsection{General remarks about semiclassics}

In this section we collect a few semiclassical formulas to be used in later chapters, in particular, in chapters 4 and 5 . For the rest of this section $\widehat{H}$ denotes a quantum Hamiltonian, whose classical limit is given by the Hamilton function $H(z)$ with $N$ degrees of freedom $z=(\mathbf{q}, \mathbf{p}) \in \mathbb{R}^{2 N}$, and $\mathbf{p} \in \mathbb{R}^{N}$ is the conjugate momenta of $\mathbf{q} \in \mathbb{R}^{N}$. 
The semiclassical limit is really a question of scales 92 . A system is in the semiclassical limit, if $\hbar$ is small compared to a characteristic action (a quantity of dimension energy $\times$ time). Hence, when we refer to the magnitude of $\hbar$, it is always implicit with respect to the natural units of the specific problem at hand. In other words, $\hbar$ is viewed as a dimensionless scaling parameter [83], separating the quantum world from the classical limit $\hbar \rightarrow 0$.

Mathematically speaking, the classical limit is singular 84, 85, 93, and does not commute with the late time limit. This has important consequences for the morphology of spectral eigenfunction (being stationary in time, they inherently involve the large time limit) 94 97, and the rich behavior of the spectral density $92,98,103$ on small energy scales, which are associated to late times. Essentially, the field of quantum chaos, where late time behavior of quantum systems with a nonintegrable classical limit are studied, is a child of this singular double limit. Whether the limiting classical dynamics is integrable, fully chaotic, or a mixture of both (as e.g. predicted by KAM's theorem 104 106 for small integrability breaking) has consequences for the quantum mechanical spectrum on small energy scales and its wave function morphology in the small $\hbar$ limit. The spectrum of a Hamiltonian and the morphology of its eigenstates are interrelated 97]. We sketch some of those results, beginning with the spectral properties, and then briefly mention results about eigenstates.

Spectral properties. The spectral density ${ }^{5} \rho(E)=\sum_{j} \delta\left(E-E_{j}\right.$ ) (summation over eigenvalues of $\widehat{H})$ can be decomposed into the mean density and correction terms as $92,95,97,107,108$

$$
\rho(E)=\langle\rho(E)\rangle+\rho_{\mathrm{osc}}(E)
$$

where

$$
\begin{aligned}
\langle\rho(E)\rangle & =(2 \pi \hbar)^{N} \int d^{2 N} z \delta(H(z)-E), \text { and } \\
d_{o s c}(E) & =\frac{1}{\hbar^{\mu+1}} \sum_{j} A_{j}(E) e^{i S_{j}(E) / \hbar}
\end{aligned}
$$

Eq. 2.4a has two important implications. First, the density of states is proportional to the size of the energy hypersurface. In particular, for one degree of freedom, $N=1$, the hypersurface is a co-dimension one subspace in a two dimensional phase space, and its size is proportional to the time that a classical orbit needs to traverse this subspace. This follows from a change of integration variables $z \mapsto \gamma(t)$

$$
\int d^{2} z \delta[H(z)-E]=\int_{\gamma} \frac{d^{2} z}{\nabla H(\gamma)}=\int_{\gamma} d t \frac{|\dot{\gamma}(t)|}{|\nabla H(\gamma(t))|}=\int_{\gamma} d t
$$

where $\gamma$ parametrizes the classical orbit at energy $E$ obeying the equation of motion $\dot{\gamma}=J \nabla H(\gamma)$. Second, it entails that the mean spacing $\langle d(E)\rangle^{-1}$ between consecutive eigenvalues scales as $\hbar^{N}$. This is an instance of the semiclassical rule that each bound state is associated to a volume of $(\hbar / 2 \pi)^{N}$ in phase space.

Eq. 2.4b is referred to as Gutzwiller's trace formula and constitutes corrections to the mean density (2.4a). These corrections are an important result of the periodic orbit theory due to Gutzwillet ${ }^{6}$ [109, 110], Balian, and Bloch 111, 112. The summation in 2.4b is over periodic

\footnotetext{
${ }^{5}$ The normalization is chosen such that $\int \rho=\operatorname{sim} \mathcal{H}$ is the number of eigenstates. In chapter 5 we use the different normalization of $\int \rho=1$, which is more common in random matrix theory and the local density of states.

${ }^{6}$ Gutzwiller's theory was developed in a series of four papers, the last two of which discuss the significance of periodic orbits for the semiclassical approximation of the density of states.
} 


\section{Mean field spin model}

orbits, counting multiple and time-reversed traversals, at energy $E$. This holds for both, integrable and fully chaotic systems, but the different nature of periodic orbits in integrable and fully chaotic systems leads to different properties of $d_{\text {osc }}$. The exponent $\mu$ equals $(N-1) / 2$ for integrable, and zero for fully chaotic (isolated periodic orbits), respectively. To every orbit, $S_{j}=\int p(q, E) d q$ is the Maupertuis's action (plus Maslov index) and its derivative w.r.t. the energy yields the orbit's period $T_{j}=S_{j}^{\prime}(E)$. Hence, Eq. (2.4b) establishes the connection between oscillations of $d(E)$ on a scale $\Delta E$ and the period $T$ of classical periodic orbits via

$$
\Delta E \sim \hbar / T
$$

(this relation is reminiscent of the 'energy time uncertainty'). Intuitively, as more terms of the series on the right hand side of $d_{\text {osc }}$ are being summed, the oscillations will lead to pronounced peaks at the position of the energy eigenvalues 7 . However, in general, $(2.4 \mathrm{~b})$ is an asymptotic series (see [97] and Refs. therein), and must be regularized, truncated, or resummed to get meaningful answers. From a practical point of view, the summation is impossible for generic non-integrable systems as the number of periodic orbits scale exponentially. Notwithstanding, Eq. 2.4b) reveals theoretical insight into the behavior on different energy scales, cf. Fig. 2.2 .

\begin{tabular}{|c|c|c|c|c|}
\hline & $\begin{array}{c}\langle d(E)\rangle \\
\text { (classical limit) }\end{array}$ & $\begin{array}{c}\text { clustering } \\
\text { (semiclassics) }\end{array}$ & $\begin{array}{l}\text { quantization } \\
\text { (non-universal) }\end{array}$ & $\begin{array}{l}\text { gap statistics } \\
\text { (universal) }\end{array}$ \\
\hline 1 & $\gg \mathcal{O}(\hbar)$ & $\mathcal{O}(\hbar)$ & $\mathcal{O}\left(\hbar^{N}\right)$ & $<\mathcal{O}\left(\hbar^{N}\right)$ \\
\hline
\end{tabular}

Figure 2.2: Schematic sketch of different regimes on various energy scales in the semiclassical limit. Small energy scales correspond to large times. The two limits of large times and small $\hbar$ do not commute, leading to rich scaling behavior.

The rich and complex structure on different energy scales $\Delta E$ as the classical limit $\hbar \rightarrow 0$ is approached, can be traced back to the fact that the long time limit and the classical limit do not commute. The details of the spectral density on a fine energy scale are related to classical orbits with a long period. Hence the semiclassical description of the spectral density involves the non-commuting double limit. Imagine $\hbar$ is small but fixed, while the resolution of the energy on scales $\Delta E$ is successively improved.

1. $\Delta E$ larger than $\hbar$ : no evidence of integrable vs. irregular. Corrections to 2.4a are averaged away. The precise form of $\langle d(E)\rangle$ depends on the details of the model and is non-universal.

2. On scales $\Delta E=\mathcal{O}(\hbar)$ : level clustering can be observed. This is a consequence of the terms $A_{j} \exp \left[S_{j}(E) / \hbar\right]$ in the summation of $d_{\text {osc }}(E)$, leading to long wavelength oscillations of $d(E)$ with wavelength $\Delta E \sim 2 \pi \hbar / T_{j}$ proportional to $\hbar$. The amplitudes $A_{j}$ decay polynomial (integrable) and exponentially (chaotic) with the number of repetitions of the orbit, leading to the fact that clustering on the $\hbar$ scale is more pronounced for integrable systems 113 than for chaotic systems. Short periodic orbits are visible as scars in the Wigner function 114 117]. These scars are suppressed by $e^{-T_{j} / h}$ [97, hence only the short orbits lead to visible scars. Despite being more suppressed, the long periodic orbits are more numerous (their number increases exponentially in $T_{j}$ ) and influence the spectrum on small energy scales (see below).

3. On scales $\Delta E=\mathcal{O}\left(\hbar^{N}\right)$ : spacing between levels start to resolve. If one wants to see the

\footnotetext{
${ }^{7}$ This intuition resembles Poisson's formula $\sum_{n \in \mathbb{Z}} \delta(E-n)=1+2 \sum_{n}^{\infty} \cos (2 \pi n E)$ (the Fourier transform of a Dirac comb on $\mathbb{Z}$ is a Dirac comb on $2 \pi \mathbb{Z}$; this underlies the Poisson resummation), where the first term on the right hand side is the mean density of the spectrum $\mathbb{Z}$ and the correction terms are the analog of $d_{\text {osc }}$.
} 
location of energy levels emerging in the summation over periodic orbits, one needs to resolve energies on a scale of $\Delta E \sim\langle d(E)\rangle^{-1} \sim 2 \pi \hbar / T$, which requires to sum orbits up to period $T \sim \int \frac{1}{h^{N-1}} \delta(E-H)$. This is particular impractical for chaotic systems, for which the number of periodic orbits increases exponentially in $1 / \hbar[95]$, and also exponentially $\sim e^{h T} /(h T)$ (where $h$ is the topological entropy of the classical system) in the period [97, 103, 118, 119]. Compare this to integrable systems, for which the number of periodic orbits with period $T$ only increases polynomially as $T^{N}$ [103]. For classically integrable models the BohrSommerfeld quantization [120] condition is an amenable criterion which does not require to sum periodic orbits. For fully chaotic systems no practical quantization condition is known.

4. On scales $\Delta E$ smaller than $\mathcal{O}\left(\hbar^{N}\right)$ one can resolve the statistics of gaps between energy levels. Typically, on this scale energies are measured in units of the local mean energy $\langle d(E)\rangle^{-1} \sim \hbar^{N}$, such that $x_{j}=E_{j}\left\langle d\left(E_{j}\right)\right\rangle$ are dimensionless and their mean density is unity. The rescaling $E_{j} \mapsto x_{j}$ is referred to as unfolding the spectrum, and amounts to pruning nonuniversal contributions to the gap statistics. The gap statistics can be used to discriminate between integrable (Poisson distributed [98, 102]), and chaotic (Wigner 8 distributed 92, 97]). The (unfolded) gap statistics (and also the spectral rigidity and the number deviation) shows universal behavior in the sense that the statistics does not depend on microscopic details, but only on the symmetry class (in particular, time reversible vs. time irreversible [103]). In the context of Billards, this was first observed by Bohigas and Giovanni [100, 101], but other examples, even in the realm of number theory (such as the gap distribution of zeros in Riemann's zeta function [103]) have been observed. This universality breaks down on energy scales larger than $\Delta E \sim \hbar^{N}$. A hint for this universality comes from the fact that long periodic orbits in fully chaotic systems obey a universal property as realized by Ozorio and Hannay 107. More precisely, for fully chaotic systems this universality can be stated in terms of the amplitudes $A_{j}=A\left(T_{j}\right)$ in Gutzwiller's formula and the density $\rho(T)=\sum_{j} \delta\left(T-T_{j}\right)$ of periodic orbits of period $T$ as 97

$$
A(T)^{2} \rho(T) \rightarrow T \text { for } T \gg \hbar^{-N}
$$

and is referred to as the classical sum rule. In words, the exponential decay of the amplitudes $A(T) \approx T \exp \left(-\frac{1}{2} h_{\mathrm{KS}} T\right)$ (where $h_{\mathrm{KS}}$ is the classical metric entropy) 97] is counteracted by the exponential number $\rho(T) \approx \exp \left(h_{\mathrm{KS}} T\right) / T$ of orbits with period $T$, such that their product equals $T$ for large periods, independent of the microscopic details of the system. The sum rule has been applied to explain the universality of the spectral rigidity 108 and the number deviation [97], see reviews [97, 103. In fact, Eq. (2.6) explains the universal statistics of any bilinear correlation in the (unfolded) spectral density on small scales $\Delta E<\hbar^{N}$. It can, however, not explain the universality of the full statistics $P(S) d S$ of the gaps $S_{j}=x_{j+1}-x_{j}$ between consecutive (unfolded) energy levels, as $P(S)$ measures correlations of all orders [122, 123]. A different argument 95, 124 explains the universal level repulsion phenomenon $P(S) \sim S$ (chaotic and time reversible, GOE statistics), and $P(S) \sim S^{2}$ (chaotic and time irreversible, GUE statistics) behavior based on a typicality argument for eigenvalues.

Spectral eigenfunctions. The fact that the classical motion in an integrable system and a fully chaotic system is different, shows up in different semiclassical behavior of the spectral Wigner function, that is the Wigner transform of the Hamiltonian eigenstates. In particular, the periodic orbits of a classically integrable system are embedded in a $(N-1)$ parameter family and are constrained to an $N$-dimensional invariant tori at a given energy $E$. The classical limit of the

\footnotetext{
${ }^{8}$ The gap statistics was first investigated by Wigner 45,121 in the context of large nuclei.
} 


\section{Mean field spin model}

spectral Wigner function at energy $E_{\mathbf{m}}$ in an integrable system is then given by 9

$$
W_{\mathbf{m}}(z)=\frac{1}{(2 \pi)^{N}} \delta\left(\mathbf{I}(z)-\mathbf{I}_{\mathbf{m}}\right)
$$

where $\mathbf{I} \in \mathbb{R}^{N}$ are conserved action variables and a function ${ }^{10}$ of the old coordinates $z=(\mathbf{q}, \mathbf{p})$, and the quantized energies $E_{\mathbf{m}}$ are labeled by the quantum numbers $\mathbf{m} \in \mathbb{N}^{N}$. To each $E_{\mathbf{m}}$ one associates the action $\mathbf{I}_{\mathbf{m}}$ via $E_{\mathbf{m}}=H\left(\mathbf{I}_{\mathbf{m}}\right)$, and the quantized set of actions $\mathbf{I}_{\mathbf{m}}$ are determined by Sommerfeld's quantization condition [120] as $\mathbf{I}_{\mathbf{m}}=\hbar(\mathbf{m}+\boldsymbol{\alpha} / 4)[92]$, where $\boldsymbol{\alpha}_{j} \in \mathbb{Z}$ is the number of real space caustic: ${ }^{11}$ encountered in the orbit $\gamma_{j}$ and related to Masolv's index). In other words, the spectral Wigner function is uniform on the invariant torus in the classical limit. In a more carefu ${ }^{12}$ semiclassical limit 96,127 the delta function is softened on a scale of $\hbar^{2 / 3}$ and $W_{\mathbf{m}}$ also has support outside and inside the invariant torus (Ref. [96] calls it a fringed torus).

In contrast, typical orbits in an ergodic system sample the full $(N-1)$-dimensional energy hypersurface uniformly. The semiclassical limit of the spectral Wigner function has been conjectured to be 94,95

$$
W(z) \propto \delta(E-H(z)) .
$$

Corrections to (2.8) constitute Wigner scars [114 117 along short classical periodic orbits, see review 97 .

We close this paragraph by remarking that $N=1$ is the special limiting case, which is trivially ergodic and trivially integrable, such that Eqs. 2.7) and (2.8) agree.

Semiclassical dynamics of Wigner functions. The semiclassical dynamics of a special class of Wigner functions associated to states that are not eigenstates of the Hamiltonian has been studied w.r.t. the distinction between integrable and fully chaotic classical limits in [96]. A systematic expansion of the quantum dynamics of general Wigner functions around the classical limit has been reviewed and developed in 88 .

\footnotetext{
${ }^{9}$ The function is correctly normalization, because $\int d^{N} \mathbf{q} d^{N} \mathbf{p} W_{\mathbf{m}}(z)=\int d^{N} \mathbf{I} d^{N} \boldsymbol{\theta} \delta\left(\mathbf{I}-\mathbf{I}_{\mathbf{m}}\right) /(2 \pi)^{N}=1$ and the canonical coordinate change $z \mapsto(\mathbf{I}, \boldsymbol{\theta})$ has unit Jacobi determinant and the $\boldsymbol{\theta}$ integration is over $[0,2 \pi]^{N}$

${ }^{10}$ The transformation from old coordinates $(\mathbf{q}, \mathbf{p})$ to the new action-angle variables $(\mathbf{I}, \boldsymbol{\theta})$ is given by $\mathbf{I}_{j}=\frac{1}{2 \pi} \int_{\gamma_{j}} \mathbf{p}(\mathbf{q})$. $d \mathbf{q}$, where $\gamma_{j}$ is the $j$ th irreducible orbit on the torus, and $\boldsymbol{\theta}=\nabla_{\mathbf{I}} S(\mathbf{q}, \mathbf{I}$,$) , where S(\mathbf{q}, \mathbf{I})=\int_{\mathbf{q}_{0}}^{\mathbf{q}} \mathbf{p}\left(\mathbf{q}^{\prime}, \mathbf{I}\right) d \mathbf{q}^{\prime}$ is Maupertuis's action.

${ }^{11}$ A real space caustic occurs when the projection $z=(\mathbf{q}, \mathbf{p}) \mapsto \mathbf{q}$ is locally not invertible.

${ }^{12}$ Essentially, by solving the $\eta$ integration in the definition of the Wigner function by a (uniform, see [125]) saddle point approximation for a WKB [126] ansatz. The structure of the critical points is such that the torus is a fold catastrophe for the Wigner function, leading to Airy-type oscillations and Airy-type exponential decay on a scale of $\hbar^{2 / 3}$ inside and outside the torus, respectively.
} 


\section{Part I}

\section{Publication Ref. [1]}





\section{Entanglement and magnetization variance in the transverse field Ising model}

This chapter elaborates on the publication [1. An exact copy of this publication is attached to this chapter in Sec. 3.8. The purpose of the following sections preceding Sec. 3.8 is twofold. First, they provide a more pedagogical and accessible introduction to the topic. In consequence, these sections present additional background information that is too elementary for a research paper, and are written more in the style of a monograph. Naturally, these sections do not contain any new results. Second, some sections contain extensions of the results of [1] that are, to the best of the author's knowledge, not published elsewhere. In particular, some results for one semiclassical degree of freedom are generalized mutatis mutandis to more degrees of freedom. Those sections that contain new unpublished material, are marked with an asterisk $(*)$. References to [1] are placed in the page margin of this treatise to ease the connection.

\subsection{Introduction}

Mean field type approximations are one of the most accessible methods to study the complexity of quantum many body systems out of equilibrium. However, the validity of such approximations has to be examined in each case. In Ref. [1] we investigate the transverse field Ising model (cf. Sec. 2.1)

$$
H=-\frac{1}{2 N} \sum_{i, j=1}^{N} s_{i}^{z} s_{j}^{z}-\Gamma \sum_{i=1}^{N} s_{i}^{x}
$$

on a finite fully connected lattice as one of the prime examples of mean field models. The mean field approximation becomes exact in the thermodynamic limit. We study the non-equilibrium dynamics after a sudden quantum quench in $\Gamma$. By exploiting the fully connected geometry, we systematically expand around the mean field limit, and compute the leading order corrections analytically. We observe that dephasing effects can quickly, on a timescale given by the square root of the system size, lead to deviations from the mean field approximation in out-of-equilibrium situations, even for large systems and away from unstable fixed points where mean field is expected to be valid.

The focus is twofold. First, we investigate the dynamics of the order parameter and its variance. The variance is inversely proportional to the system size and therefore constitutes a correction to mean field. Based on the dynamics of the variance we identify four qualitative different regions in the dynamical phase diagram that cannot be distinguished by solely looking at the mean of the order parameter. In particular, we find a phase of periodically enhanced spin squeezing and spreading and explain the phenomenon as a consequence of dephasing between nearby classical periodic orbits.

Second, we investigate the entanglement with respect to a bipartition of the lattice. An analytic expression for the leading contribution of the family of Rényi entanglement entropies is given and the close connection to spin squeezing is confirmed quantitatively. Remarkably, in contrast to the spin variance, the entanglement entropies do not vanish in the mean field limit. The discussion of the dynamical phase diagram based on the order parameter and its variance translates directly to a 


\section{Entanglement and magnetization variance in the transverse field Ising model}

discussion of the order parameter independent entanglement entropy. In particular, entanglement entropy grows logarithmically in time for quenches in the regime of periodically enhanced squeezing.

The calculations are facilitated by the mapping of the fully connected spin model (3.1) to the effective one-dimensional model (cf. Sec. 2.2)

$$
H_{\mathrm{eff}}(x, p)=-\frac{1}{2}(x-1 / 2)^{2}-\Gamma \sqrt{x-x^{2}} \cos (p),
$$

where $\hbar_{\mathrm{eff}}=1 / N$ and $p=-i \hbar_{\mathrm{eff}} \partial_{x}$ is the conjugate momentum of a fictitious particle whose position $x=N_{+} / N$ is the fraction of up spins. The thermodynamic limit $N \rightarrow \infty$ in 3.1 translates to the classical limit $\hbar_{\mathrm{eff}} \rightarrow 0$ in $(3.2)$. We use semiclassical techniques to compute semiclassical corrections to the classical limit in the effective model, in order to infer corrections to the mean field limit in the spin model (3.1).

Even though the Ref. [1] focuses on the specific model of the transverse field Ising model, the used methods only rely on the fact that the model facilitate a semiclassical effective description. In this sense, our findings are universal and translate to other models that permit a semiclassical effective description.

\subsection{Hierarchy of cumulants $*$}

The publication [1] focuses on the quench induced dynamics of the order parameter and its variance.

see

Sec.

and appendix B of 1

Eq. (3) in

[1]

This section elaborates on a generalization of the techniques to higher order cumulant: 1 . One of the main results in this respect is Eq. (3.13).

In the following we explain how the time evolution of the cumulants of a narrow wave packet

$$
\psi(x, t)=e^{-N f(x, t)}
$$

is obtained from the Schrödinger equation

$$
\frac{i}{N} \frac{d}{d t} \psi(x, t)=H_{\mathrm{eff}}(x, p) \psi(x, t)
$$

in the semiclassical limit $1 / N \rightarrow 0$. The analysis is akin to the wave packet dynamics by Heller [89, 128] and time-dependent WKB techniques [129, 130] in which the Planck constant is replaced by $1 / N$. One of the main results is that, to leading order in $1 / N$, the dynamics of the cumulants is given by a hierarchical system of differential equations such that the higher cumulants only couple to lower cumulants, see Eq. (3.13) below. We stress that Eq. 3.13 holds generically, independent of the precise form of the Hamiltonian. This result is then applied to the effective model (3.2).

\subsubsection{Expansion of cumulants at fixed time}

Prior to the discussion of the time evolution, we want to establish the connection between the complex rate function $f(x, t)$ and the cumulants of the probability density $|\psi(x, t)|^{2}$ at a fixed time. To simplify the notation, we do not write the time dependence explicitly.

Let us assume that $|\psi(x)|^{2}=e^{-N 2 \Re f(x)}$ is concentrated around a global maximum at $x_{\mathrm{cl}}$ such that the real part of $f$ has a global minimum at $x_{\mathrm{cl}}$. We expand $f(z)$ around this minimum in a Taylor expansion,

$$
f(x)=\sum_{n=0} \frac{f_{n}}{n !}\left(x-x_{\mathrm{cl}}\right)^{n}, \quad \text { with } \quad f_{n}=\left.\frac{\partial^{n} f}{\partial x^{n}}\right|_{x_{\mathrm{cl}}} .
$$

\footnotetext{
${ }^{1}$ The author acknowledges discussions with Markus Oberthaler, Thomas Gasenzer, and Wolfgang Müssel at the University of Heidelberg.
} 
Real and imaginary part of $f$ are denoted by $g$ and $-\theta$, respectively, and $f_{n}=g_{n}-i \theta_{n}$. Because of the choice of $x_{\mathrm{cl}}$ one has $g_{1}=0$. Moreover, we can set $f_{0}=0$ by introducing the normalization constant $\mathcal{N}=\int d z e^{-N 2 g(x)}$. Before we compute the cumulants, we need to compute the $n$th moment

$$
\left\langle\left(x-x_{\mathrm{cl}}\right)^{n}\right\rangle=\mathcal{N}^{-1} \int d x\left(x-x_{\mathrm{cl}}\right)^{n} e^{-N 2 g(x)} .
$$

The moments are obtained by differentiating the moment generating function $Z(j)=\mathcal{N}^{-1} \int d z e^{-N 2 g(z)+j\left(x-x_{\mathrm{cl}}\right)}$ w.r.t. the current $j$. For large $N$ the integral can be computed by a saddle point approximation 131]. To this end the exponent is split into a quadratic part $j \bar{x}-N g_{2} \bar{x}^{2}$ and the rest $V(\bar{z})=\sum_{n \geqslant 3} 2 g_{n} \bar{x}^{n} / n$ !, $\bar{x}$ being $\left(x-x_{\mathrm{cl}}\right)$. The function $Z(j)$ is computed formally by solving the 'non-interacting' Gaussian integral.

$$
\begin{aligned}
Z(j) & =\mathcal{N}^{-1} \int d \bar{x} e^{-N V(\bar{x})} e^{j \bar{x}-N g_{2} \bar{x}^{2}} \\
& =\mathcal{N}^{-1} e^{-N V\left(\partial_{j}\right)} \int d \bar{x} e^{j \bar{x}-N g_{2} \bar{x}^{2}} \\
& \propto \mathcal{N}^{-1} e^{-N V\left(\partial_{j}\right)} e^{\frac{1}{2} j^{2} /\left(2 g_{2} N\right)}
\end{aligned}
$$

The moments are then obtained perturbatively from the last expression by expanding $e^{-N V\left(\partial_{j}\right)}$ in a Taylor series. Based on these moments one gets the first cumulants. A tedious calculation, facilitated by the use of a computer algebra system (Mathematica), yields

$$
\begin{aligned}
\kappa_{1} & =\langle x\rangle=x_{\mathrm{cl}}+\mathcal{O}(1 / N) \\
\kappa_{2} & =\frac{1}{2 g_{2} N}+\mathcal{O}\left(1 / N^{2}\right) \\
\kappa_{3} & =-\frac{2 g_{3}}{\left(2 g_{2}\right)^{3} N^{2}}+\mathcal{O}\left(1 / N^{3}\right), \\
\kappa_{4} & =-\frac{2 g_{4}}{\left(2 g_{2}\right)^{4} N^{3}}+\frac{12 g_{3}^{2}}{\left(2 g_{2}\right)^{5} N^{3}}+\mathcal{O}\left(1 / N^{4}\right) .
\end{aligned}
$$

Equations $3.7 \mathrm{~b}$ to $3.7 \mathrm{~d}$ ) can be represented diagrammatically as

$$
\begin{aligned}
& \kappa_{2}=-+\mathcal{O}\left(1 / N^{2}\right), \\
& \left.\kappa_{3}=2\right\rangle+\mathcal{O}\left(1 / N^{3}\right), \\
& \left.\left.\kappa_{4}=2\right\rangle+12\right\rangle\left\langle+\mathcal{O}\left(1 / N^{4}\right) .\right.
\end{aligned}
$$

We remark a few basic facts about the diagrammatic expansion. (i) All vacuum bubbles (i.e. diagrams without external legs) are canceled by the normalization constant $\mathcal{N}$ in the denominator of Eq. (3.6). (ii) Every internal and external leg contributes a factor of $1 /\left(2 g_{2} N\right)$, whereas a vertex with $n$ legs contributes $\left(-g_{n} N\right)$. (iii) Consequently, a loop consisting of an equal number of vertices and internal lines is suppressed by $1 / N$ compared to a single vertex substituting the loop. An expansion in $1 / N$ is an expansion in loops. (iv) Hence, the leading order term in $1 / N$ of the moments is given by tree diagrams (i.e. diagrams without loops). (v) Cumulants are represented by connected diagrams. The dominant contribution of the $n$th cumulant is thus given by connected tree diagrams with $n$ external legs, compare Eq. 3.8 . It follows that $\kappa_{n}$ scales as $\mathcal{O}\left(1 / N^{n-1}\right)$ and that, to leading order, $\kappa_{n}$ depends only on $g_{m}$ with $m \leqslant n$.

Obviously, the cumulants $\kappa_{n}$ depend only on the real part of the rate function $f(x)$. The imaginary part $\theta(x)$ determines the cumulants of the conjugate momentum operator $p=-i \partial_{x} / N$. 
3 Entanglement and magnetization variance in the transverse field Ising model

In particular, to leading order in $1 / N$, the expectation value of $\langle p\rangle$ is 81

$$
\langle p\rangle=\mathcal{N}^{-1} \int d x\left(i g^{\prime}(x)+\theta^{\prime}(x)\right) e^{-N 2 g(x)}=\theta_{1}+\mathcal{O}(1 / N)=p_{\mathrm{cl}}+\mathcal{O}(1 / N) .
$$

Analogously to Eq. (3.7a), we denote $\theta_{1}$ by $p_{\mathrm{cl}}$. Similarly, the higher cumulants of $p$ depend on higher order coefficients $\theta_{n}$ and can be computed as explained above.

\subsubsection{Time dependence of the cumulants}

In this section we discuss the time evolution of $\kappa_{n}$ and prove two facts. First, the leading order of the expectation values $\langle x\rangle$ and $\langle p\rangle$ obey the classical Hamilton equations of motion

$$
\dot{x}_{\mathrm{cl}}(t)=\frac{\partial H_{\mathrm{eff}}}{\partial p_{\mathrm{cl}}}, \quad \text { and } \quad \dot{p}_{\mathrm{cl}}(t)=-\frac{\partial H_{\mathrm{eff}}}{\partial x_{\mathrm{cl}}} .
$$

This was already noted in [81]. And second, the leading order of the higher cumulants $\kappa_{n}$ obey a hierarchical system of differential equations such that the higher cumulants only couple to lower cumulants.

cf. Eq. (6) and appendix B in [1]
$3.13 \mathrm{a}$ is equivalent to Eq. (7) in [1].

The Schrödinger Eq. (3.4) imposes the nonlinear partial differential equation (PDE)

$$
\partial_{t} f(x, t)=i H_{\mathrm{eff}}\left(x, i \partial_{x} f(x, t)\right)+\mathcal{O}(1 / N)
$$

on the rate function. As a consequence, the minimum $x_{\mathrm{cl}}(t)$ of $\Re f(x, t)$ and the coefficients $f_{n}(t)=$ $\left.\partial_{x}^{n} f(x, t)\right|_{x_{\mathrm{cl}}(t)}$ in the Taylor series 3.5 are time-dependent. Equation 3.11 induces the ordinary differential equation (ODE)

$$
\begin{aligned}
\frac{d}{d t} f_{n}=\frac{d}{d t}\left(\left.\partial_{x}^{n} f\right|_{x_{\mathrm{cl}}(t)}\right) & =\left.i \partial_{x}^{n} H_{\mathrm{eff}}\left(x, i \partial_{x} f\right)\right|_{x_{\mathrm{cl}}(t)}+\left.\partial_{x}^{n+1} f\right|_{x_{\mathrm{cl}}(t)} \dot{x}_{\mathrm{cl}} \\
& =\left.i \partial_{x}^{n} H_{\mathrm{eff}}\left(x, i \partial_{x} f\right)\right|_{x_{\mathrm{cl}}(t)}+f_{n+1} \dot{x}_{\mathrm{cl}} .
\end{aligned}
$$

on the coefficients $f_{n}$. In particular, for $n=1$ we obtain $\dot{f}_{1}=i H_{\mathrm{eff}}^{(1,0)}\left(x_{\mathrm{cl}}, i f_{1}\right)-H_{\mathrm{eff}}^{(0,1)}\left(x_{\mathrm{cl}}, i f_{1}\right) f_{2}+$ $f_{2} \dot{x}_{\mathrm{cl}}(t)$. Here $H_{\text {eff }}^{(n, m)}$ denotes the $n$th and $m$ th derivative of $H_{\text {eff }}$ w.r.t. its first and second argument, respectively. By definition, $g_{1}=0$ and hence $i f_{1}=\theta_{1}=p_{\mathrm{cl}}$, see Eq. (3.9). Thus,

$$
-i \dot{p}_{\mathrm{cl}}(t)=i H_{\mathrm{eff}}^{(1,0)}\left(x_{\mathrm{cl}}, p_{\mathrm{cl}}\right)-H_{\mathrm{eff}}^{(0,1)}\left(x_{\mathrm{cl}}, p_{\mathrm{cl}}\right) f_{2}+f_{2} \dot{x}_{\mathrm{cl}}(t)
$$

Considering the real and imaginary part of this equation separately, yields that $x_{\mathrm{cl}}(t)$ and $p_{\mathrm{cl}}(t)$ fulfill the classical equations of motion (3.10). Note that the dependence on $f_{2}$ cancels.

Now, we look at Eq. (3.12) for $n \geqslant 2$. The right hand side only seemingly depends on $f_{n+1}$. In fact, the only term in the expression $\left.i \partial_{x}^{n} H_{\mathrm{eff}}\left(x, i \partial_{x} f\right)\right|_{x_{\mathrm{cl}}(t)}$ that contains $f_{n+1}$ is

$$
-H_{\mathrm{eff}}^{(0,1)}\left(x_{\mathrm{cl}}, p_{\mathrm{cl}}\right) f_{n+1} \text {. }
$$

This term is canceled by the term $f_{n+1} \dot{x}_{\mathrm{cl}}(t)$ due to the equations of motion. Thus, the differential equation (3.12) of $f_{n}$ only depends on $f_{m}$ with $m \leqslant n$. As the $n$th cumulant is a function of the first $(n-1)$ coefficients $g_{2}, \ldots g_{n}$ (see Eqs. (3.7b) to (3.7d) and the subsequent discussion) the coupling among the cumulants obeys the same hierarchy as the $f_{n}$ 's.

We state the system of ODEs for the first four cumulants explicitly. The equations are obtained with the help of a computer algebra system (Mathematica): 


$$
\begin{aligned}
\partial_{t} \kappa_{2} & =A_{2} \kappa_{2}, \\
\partial_{t} \kappa_{3} & =A_{3} \kappa_{2}^{2}+\frac{3}{2} A_{2} \kappa_{3}+C_{3}, \\
\partial_{t} \kappa_{4} & =A_{4} \kappa_{2}^{3}+4 A_{3} \kappa_{2} \kappa_{3}+2 A_{2} \kappa_{4}+D_{4} \kappa_{2},
\end{aligned}
$$

where

$$
\begin{aligned}
& A_{2}=2 \theta_{2} H_{\mathrm{eff}}^{(0,2)}+2 H_{\mathrm{eff}}^{(1,1)}, \\
& A_{3}=3 \theta_{2}^{2} H_{\mathrm{eff}}^{(0,3)}+6 \theta_{2} H_{\mathrm{eff}}^{(1,2)}+3 \theta_{3} H_{\mathrm{eff}}^{(0,2)}+3 H_{\mathrm{eff}}^{(2,1)}, \\
& A_{4}=4 \theta_{2}^{3} H_{\mathrm{eff}}^{(0,4)}+12 \theta_{2}^{2} H_{\mathrm{eff}}^{(1,3)}+12 \theta_{2} H_{\mathrm{eff}}^{(2,2)}+12 \theta_{3} \theta_{2} H_{\mathrm{eff}}^{(0,3)}+12 \theta_{3} H_{\mathrm{eff}}^{(1,2)}+4 \theta_{4} H_{\mathrm{eff}}^{(0,2)}+4 H_{\mathrm{eff}}^{(3,1)}, \\
& C_{3}=-H_{\mathrm{eff}}^{(0,3)} / 4 N^{2}, \\
& D_{4}=-\theta_{2} H_{\mathrm{eff}}^{(0,4)} / N^{2}-H_{\mathrm{eff}}^{(1,3)} / N^{2},
\end{aligned}
$$

and

$$
\begin{aligned}
\partial_{t} \theta_{2}= & -\theta_{2}^{2} H_{\mathrm{eff}}^{(0,2)}-2 \theta_{2} H_{\mathrm{eff}}^{(1,1)}+\frac{H_{\mathrm{eff}}^{(0,2)}}{4 \kappa_{2}^{2} N^{2}}-H_{\mathrm{eff}}^{(2,0)}, \\
\partial_{t} \theta_{3}= & \theta_{2}^{3}\left(-H_{\mathrm{eff}}^{(0,3)}\right)-3 \theta_{2}^{2} H_{\mathrm{eff}}^{(1,2)}+\theta_{3}\left(-3 \theta_{2} H_{\mathrm{eff}}^{(0,2)}-3 H_{\mathrm{eff}}^{(1,1)}\right) \\
& +\theta_{2}\left(\frac{3 H_{\mathrm{eff}}^{(0,3)}}{4 \kappa_{2}^{2} N^{2}}-3 H_{\mathrm{eff}}^{(2,1)}\right)+\frac{3 H_{\mathrm{eff}}^{(1,2)}}{4 \kappa_{2}^{2} N^{2}}-\frac{3 \kappa_{3} H_{\mathrm{eff}}^{(0,2)}}{4 \kappa_{2}^{4} N^{2}}-H_{\mathrm{eff}}^{(3,0)}, \\
\partial_{t} \theta_{4}= & \theta_{2}^{4}\left(-H_{\mathrm{eff}}^{(0,4)}\right)-4 \theta_{2}^{3} H_{\mathrm{eff}}^{(1,3)}-3 \theta_{3}^{2} H_{\mathrm{eff}}^{(0,2)}+\theta_{4}\left(-4 \theta_{2} H_{\mathrm{eff}}^{(0,2)}-4 H_{\mathrm{eff}}^{(1,1)}\right) \\
& -\frac{H_{\mathrm{eff}}^{(0,4)}}{16 \kappa_{2}^{4} N^{4}}+\theta_{2}^{2}\left(\frac{3 H_{\mathrm{eff}}^{(0,4)}}{2 \kappa_{2}^{2} N^{2}}-6 H_{\mathrm{eff}}^{(2,2)}\right)+\theta_{2}\left(\frac{3 H_{\mathrm{eff}}^{(1,3)}}{\kappa_{2}^{2} N^{2}}-\frac{3 \kappa_{3} H_{\mathrm{eff}}^{(0,3)}}{\kappa_{2}^{4} N^{2}}-4 H_{\mathrm{eff}}^{(3,1)}\right) \\
& +\theta_{3}\left(-6 \theta_{2}^{2} H_{\mathrm{eff}}^{(0,3)}-12 \theta_{2} H_{\mathrm{eff}}^{(1,2)}+\frac{3 H_{\mathrm{eff}}^{(0,3)}}{2 \kappa_{2}^{2} N^{2}}-6 H_{\mathrm{eff}}^{(2,1)}\right) \\
& +\frac{15 \kappa_{3}^{2} H_{\mathrm{eff}}^{(0,2)}}{4 \kappa_{2}^{6} N^{2}}+\frac{3 H_{\mathrm{eff}}^{(2,2)}}{2 \kappa_{2}^{2} N^{2}}-\frac{3 \kappa_{3} H_{\mathrm{eff}}^{(1,2)}}{\kappa_{2}^{4} N^{2}}-\frac{\kappa_{4} H_{\mathrm{eff}}^{(0,2)}}{\kappa_{2}^{5} N^{2}}-H_{\mathrm{eff}}^{(4,0)} .
\end{aligned}
$$

$H_{\text {eff }}^{(n, m)}$ denotes the $n$th and $m$ th derivative of $H_{\text {eff }}$ w.r.t. its first and second argument, respectively, evaluated at the classical orbit $\left(x_{\mathrm{cl}}, p_{\mathrm{cl}}\right)$.

Decoupling of Scales. The fact that the dynamics of $\kappa_{n}$ is independent of $\kappa_{m}$ with higher order $m>n$ in the large $N$ limit is a remarkable and non-trivial result. The derivation relies on the fact that the mean $\langle x\rangle$ evolves along a classical trajectory obeying Hamilton's equations of motion. Using the equations of motion in the ODE for $f_{n}$ yields exact canceling of higher order terms proportional to $f_{n+1}$, which could in principal couple to the flow of $f_{n}$. In the large $N$ limit the influence among the cumulants is a one way rode in the direction of increasing order. The (nonrescaled) cumulants and moments live on different scales. More precisely, the $n$th cumulant is of order $\mathcal{O}\left(N^{-(n-1)}\right)$. Thus, the dynamics of $(3.13)$ happens on decoupled scales of different powers of $N$. For example, the dynamics on scale $N^{-(n-1)}$ is only influenced by the dynamics on coarser scales $N^{-(m-1)}$ with $m<n$, but is not influenced by the cumulants resolving finer scales.

To appreciate this result, we give the following interpretation. Given an initial state of the form 


\section{Entanglement and magnetization variance in the transverse field Ising model}

(3.3) with rate function $f_{t=0}(x)$, and solution $f_{t}(x)$ of (3.11). From $f_{t}(x)$ one can compute the scaling $\operatorname{limit} \lim _{N} N^{n-1} \kappa_{n}$ of the $n$th cumulant, cf. Eq. (3.7). Now imagine, we change the initial data $f_{t=0}(x)$ of the rate function to $\widetilde{f}_{t=0}(x)$ in such a way that the first $n$ cumulants at time $t=0$ agree with the unperturbed cumulants, i.e. $N^{m-1} \widetilde{\kappa}_{m}(t=0)=N^{m-1} \kappa_{m}(t=0)$ for all $m \leq n$ in the limit $N \rightarrow \infty$. Then, this equality of the first $n$ rescaled cumulants persists for all times. Hence, perturbing the initial data of the PDE (3.11) weakly (in the sense that the first $n$ cumulants are unchanged), leads to a new solution that cannot be distinguished from the unperturbed solution by just looking at the first $n$ cumulants in the scaling limit $N \rightarrow \infty$.

This decoupled hierarchy of scales is only exact in the scaling limit $N \rightarrow \infty$ and for initial states of the form (3.3). For finite $N$ this decoupling can only hold approximately, and on early time scales (an analysis of the time scale of validity is given in Sec. 4.5 of [1]). This can also be seen perturbatively, by looking at corrections to the cumulants. So, despite the fact that the scaling limit of the cumulants $\kappa_{n}$ depends only on $f_{m}$ with $m \leq n$, correction terms to $\kappa_{n}$ also depends on higher order $f_{m}$ with $m>n$. Metaphorically speaking, the correction terms to the cumulants 'destroy' the one-way influence from lower order to higher order. Going beyond the perturbative expansion, that is, when the full PDE (3.11) is solved for an initial rate function $f_{t=0}(x)$ and the cumulants obtained from the solution $f_{t}(x)$ are not viewed in the scaling limit, there is, of course, no reason to expect that the higher order cumulants do not influence lower cumulants.

\subsubsection{Large deviation form of the initial coherent state}

cf. Eq. (11)

In Sec. 4 of [1], the (quadratic approximation of the) rate function of the pre-quench ground state in [1]

was given. In this section we explain how to obtain the initial rate function of the effective one particle wave function $\psi_{0}(z)$ corresponding to the coherent spin state

$$
\left|\Psi_{0}\right\rangle=\sum \psi_{0}(z)|z\rangle=|\theta, \phi\rangle \equiv \sum_{k=0}^{N}\left(\begin{array}{c}
N \\
k
\end{array}\right)^{1 / 2}(\cos \theta / 2)^{k}\left(e^{i \phi} \sin \theta / 2\right)^{N-k}|z=2 k / N-1\rangle
$$

$\left(|z\rangle\right.$ denotes the eigenstate of the $N / 2$-pseudo-spin $\widehat{J}_{z}$ with eigenvalue $N z / 2$ ). We need to find the large deviation form of the coefficient $\psi_{0}(z)=\langle z \mid \theta, \phi\rangle$ in the limit $N, k \gg 1$. Approximating the binomial in Eq. (3.14) using Stirling's formula, yields

$$
\psi(x) \asymp e^{-N f(x)}, \quad f(x)=\frac{x}{2} \log (\tan \theta / 2)+\frac{1}{4} \log (x+1)^{x+1}(1-x)^{1-x}+i \phi x / 2 .
$$

Note that the imaginary part $\Im f=\phi x / 2$ is linear in $z$ such that $p_{\mathrm{cl}}=\theta_{1}=-\phi$ and $\theta_{n}=0$ for $n \geqslant 2$. The real part $\Re f$ has a minimum at $x_{\mathrm{cl}}=\cos \theta$ and the initial cumulants are obtained by expanding $\Re f$ around this minimum, see Eqs. $3.7 \mathrm{~b})$ to $(3.7 \mathrm{~d})$.

In particular, when the coherent state is prepared on the unstable fixed point at $\theta=\pi / 2$ and $\phi=\pi$, one obtains the initial conditions

$$
\begin{aligned}
x_{\mathrm{cl}}(0) & =0, \\
p_{\mathrm{cl}}(0) & =\pi, \\
\kappa_{2}(0) & =1 / N, \\
\kappa_{3}(0) & =0, \\
\kappa_{4}(0) & =-2 / N^{3}, \\
\theta_{n \geqslant 2}(0) & =0, \quad \text { for } n \geqslant 2 .
\end{aligned}
$$


a)

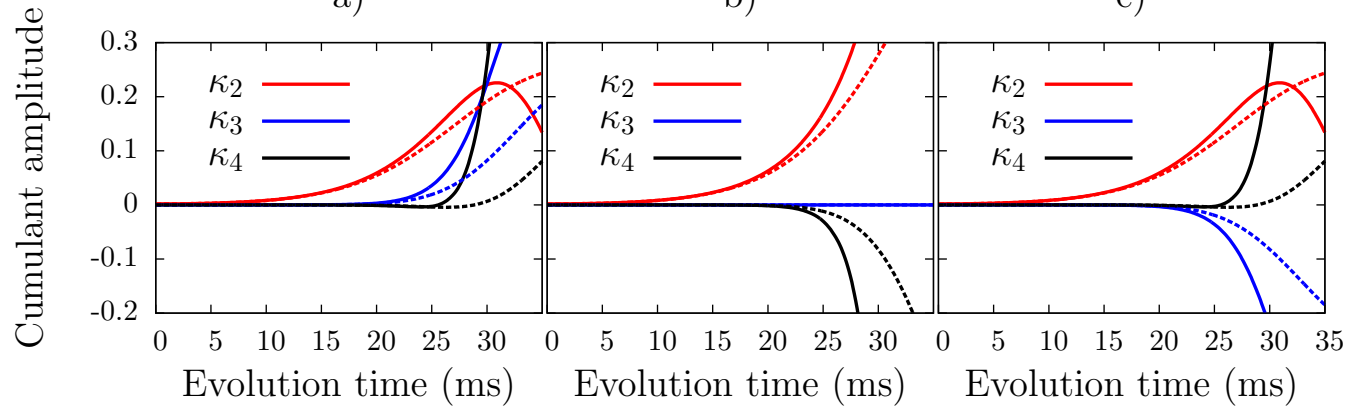

Figure 3.1: Solid curves, early time evolution of the cumulants $\kappa_{2}, \kappa_{3}, \kappa_{4}$ obtained by numerically integrating Eq. (3.13) with initial condition (3.15) for different detunings $\delta / \Omega=-0.05$ (a), $\delta / \Omega=0$ (b), $\delta / \Omega=+0.05$ (c), $N=540$, and $\Lambda=1.62$. Dashed curves, exact diagonalization results of the full Schrödinger Eq. $i \partial_{t}|\Psi\rangle=\left(\chi J_{z}^{2}-\Omega J_{x}+\delta J_{z}\right)|\Psi\rangle$ with initial condition (3.14).

\subsubsection{Cumulants in $z$ direction}

We discuss the ODEs $(3.13)$ for the Hamiltonian ${ }^{2}$

$$
H_{\mathrm{eff}}(z, p)=\frac{\Lambda}{4} z^{2}-\frac{1}{2} \sqrt{1-z^{2}} \cos (2 p)+\frac{\delta}{2 \Omega} z .
$$

Initially, the cumulants are small as they are suppressed by at least one factor of $1 / N$, see Eq. (3.15). On an early time scale the cumulants built up successively in time. This follows from the hierarchical structure of Eq. (3.13).

Let us consider the asymmetric case $\delta \neq 0$ (Fig. 3.1a,c). Equation $3.13 \mathrm{a}$ ) shows that the early time increase of $\kappa_{2}$ is caused by the positive coefficient $A_{2}$. The growth of $\kappa_{2}$ influences the behavior of $\kappa_{3}$ through the term $A_{3} \kappa_{2}^{2}$ on the right hand side of Eq. $3.13 \mathrm{~b}$. Depending on the sign of $\delta$, the coefficient $A_{3}$ is either positive $(\delta>0)$ or negative $(\delta<0)$, such that $\kappa_{3}$ either increases or decreases. The very early time evolution of the fourth cumulant is dominated by the term $A_{4} \widetilde{\kappa}_{2}^{3}$ on the right hand side of Eq. (3.13c). Because the coefficient $A_{4}$ is negative, $\kappa_{4}$ initially decreases. As time proceeds and the modulus of $\kappa_{3}$ grows, the term $4 A_{3} \kappa_{2} \kappa_{3}$ starts to dominate the right hand side of Eq. (3.13c). Since $4 A_{3} \kappa_{2} \kappa_{3}$ is positive (independent of the sign of $\delta$ ), the fourth cumulant then increases and becomes positive.

In the symmetric case, $\delta=0$, the coefficients $A_{3}$ and $C_{3}$ remain zero, so that $\kappa_{3}=0$. When $\kappa_{3}=0$, the dynamics of the fourth cumulant is dominated by the single term $A_{4} \kappa_{2}^{3}$ with $A_{4}<0$. As a consequence, $\kappa_{4}$ decreases and remains negative (Fig. 3.1b).

\subsubsection{Cumulants in $y$ direction}

The BEC Hamiltonian (3.16) can also be expanded in the basis of the pseudo-spin eigenstates in $y$ direction. Analogously to Eq. (3.16), the resulting effective Hamiltonian is then

$$
H_{\text {eff }}(y, p)=\frac{\Lambda}{8}\left(1-y^{2}\right)(1+\cos 4 p)-\frac{1}{2} \sqrt{1-y^{2}} \sin 2 p-\frac{\delta}{2 \Omega} \sqrt{1-y^{2}} \cos 2 p
$$

\footnotetext{
${ }^{2}$ This is the BEC-formulation of the effective spin Hamiltonian 3.2 , see e.g. 46 65.
} 
a)

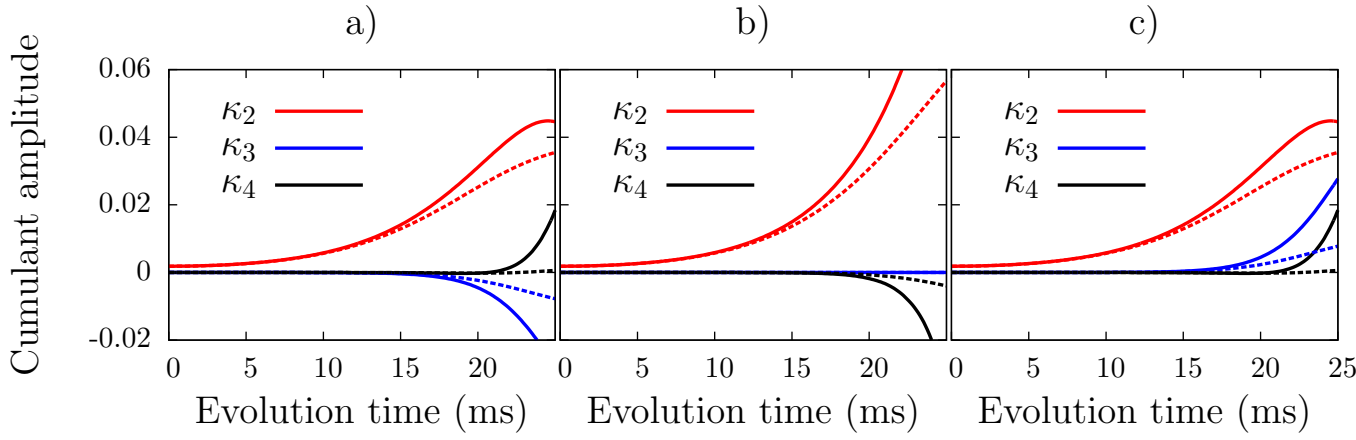

Figure 3.2: Same as Fig. 3.1 for the first cumulants in $y$ direction.

where $p=-i \partial_{y} / N$ is the conjugate variable of $y$. Analogously to the discussion of the cumulants in $z$ direction, the cumulants in $y$ direction are described by Eq. (3.13) with Hamiltonian (3.17). It turns out that the early time dynamics of the cumulants in $y$ direction is qualitatively similar to the cumulants in $z$ direction (compare Fig. 3.2).

To summarize, the quantitative time evolution of the cumulants depends on the precise form of the Hamiltonian. Though, the fact that higher cumulants built up successively on the early time scale is a universal observation, independent of the Hamiltonian details.

\subsection{Bipartite entanglement}

We consider the entanglement of permutation invariant states $|\Psi\rangle=\sum \Psi\left(N_{+}\right)\left|N_{+}\right\rangle$of $N$ spins in $\mathcal{D}_{N}$ w.r.t. a bipartition into two disjoint sets of $A$ and $B$ spins such that $A+B=N$. The Dicke subspaces of permutation invariant states of the spins corresponding to the two subsets are denoted by $\mathcal{D}_{A}$ and $\mathcal{D}_{B}$, respectively ${ }^{3}$. The relative subsystem sizes are denoted by $\alpha=A / N$ and

cf. (14) in $\beta=B / N$, respectively. The bipartite state can be expanded as

$$
|\Psi\rangle=\sum_{A_{+}, B_{+}} \underbrace{\Psi\left(A_{+}+B_{+}\right) \sqrt{\left(\begin{array}{c}
N_{A} \\
A_{+}
\end{array}\right)\left(\begin{array}{l}
N_{B} \\
B_{+}
\end{array}\right) /\left(\begin{array}{c}
N \\
A_{+}+B_{+}
\end{array}\right)}}_{\Psi_{A B}\left(A_{+}, B_{+}\right)}\left|A_{+}, B_{+}\right\rangle,
$$

in the Dicke product basis $\left|A_{+}, B_{+}\right\rangle$of $\mathcal{D}_{A} \otimes \mathcal{D}_{B}$. The simple form of the coefficients $\Psi_{A B}\left(A_{+}, B_{+}\right)$ in front of $\left|A_{+}, B_{+}\right\rangle$as a function of $\Psi\left(A_{+}+B_{+}\right)$is a consequence of the permutation invariance of $|\Psi\rangle$.

Many entanglement measure of $|\Psi\rangle$, such as the entanglement Hamiltonian $H_{E}=-\log \rho_{A}$ and the Rényi entanglement entropies $S_{A}=\frac{1}{1-n} \log \rho_{A}^{n}$, are functions of the reduced density matrix $\rho_{A}=\operatorname{Tr}_{B}|\Psi\rangle\langle\Psi|$. In general, the smaller Hilbert space dimension of the subsystem Hilbert spaces $\mathcal{H}_{A}$ and $H_{B}$ gives the upper bound $\log \min \left(\operatorname{dim} \mathcal{H}_{A}, \mathcal{H}_{B}\right)$ on all Rényi entanglement entropies. As the dimension of the Dicke subsystem $\mathcal{D}_{A}$ is $(A+1)=\alpha N+1$, i.e. linear in the number of spins, the upper bound

$$
S_{A}^{\text {upper bound }}=\log (\alpha N)+\mathcal{O}(1 / N)
$$

(assuming for the sake of concreteness that $A \leq B$ ) on the entanglement entropy scales logarithcf. Sec. 5.1 mically in the system size $N$.

of 1

\footnotetext{
${ }^{3}$ By a slight abuse of notation, $A$ and $B$ denote the subsystems, as well as the number of spins in each respective subsystems.
} 
In the case of a bipartite Gaussian pure state $|\Psi\rangle$ with inverse covariance $\Gamma_{A B}$, both, the Rényi entanglement entropies, as well as the entanglement Hamiltonian, follow from the covariance matrix 4 $\Sigma_{A}$ of the reduced density matrix $\rho_{A}$. There are two ways to obtain $\Sigma_{A}$ from $\Gamma_{A B}$, corresponding to the two paths from $\Gamma_{A B}$ to $\Sigma_{A}$ in the following diagram.

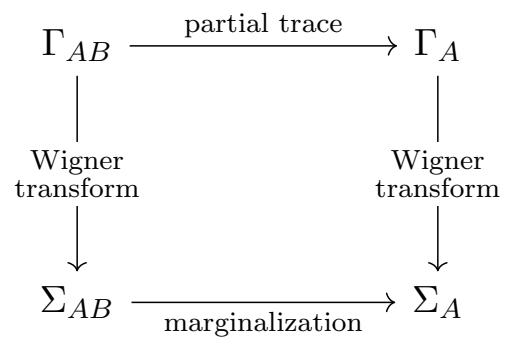

First, computing $\rho_{A}$ and then its Wigner function $W_{A}$. And second, computing the Wigner function $W_{A B}$ of $|\Psi\rangle$, and then its marginal $W_{A}=\int_{B} W_{A B}$. Since the above diagram commutes, both ways yield the same result. However, in the Gaussian case going from $W_{A B}$ to $W_{A}$ is considerably easier than going from $|\Psi\rangle$ to $\rho_{A}$. The covariance of $W_{A}$ follows form the covariance of $W_{A B}$ by simply crossing out the rows and columns of $\Sigma_{A B}$ associated to the dimensions of $B$. In contrast, the covariance of $\rho_{A}$ follows from $\Gamma^{A B}$ by Eq. (48) of [1], which is more complicated.

Entanglement Hamiltonian. Employing semiclassical techniques, we have also derived the full entanglement Hamiltonian $H_{E}=-\log \rho_{A}$ corresponding to the reduced density matrix. To leading order as $N \rightarrow \infty$ when the large deviation state is approximated as a Gaussian wave function (this amounts to approximating the unimodal rate function to second order around its minimum) the entanglement Hamiltonian is a harmonic oscillator with equidistant spectrum. The relation between the covariance $\Sigma_{A}$ of the Gaussian reduced density matrix $\rho_{A}$ and the two by two quadratic form $V$ of the harmonic oscillator entanglement Hamiltonian $H_{E}=\frac{1}{2}(\widehat{x}, \widehat{p}) V(\widehat{x}, \widehat{p})$ is given by

$$
V=2 \sqrt{\operatorname{det} \Sigma_{A}} \operatorname{arctanh}\left[\left(2 \sqrt{\operatorname{det} \Sigma_{A}}\right)^{-1}\right]\left(\Sigma_{A}\right)^{-1} .
$$

This relation has been generalized to higher dimensions in appendix A. More explicitly, for a reduced Gaussian density matrix $\rho_{A}$ on $d_{A}$-dimensional reduced state space with covariance matrix $\Sigma_{A}$, the entanglement Hamiltonian $H_{E}=-\log \rho_{A}$ is a harmonic oscillator with $2 d_{A}$ by $2 d_{A}$ quadratic form $V$, and, cf. A.4,

$$
\Sigma_{A}^{-1}=-2 J|J V| \tan \left(\frac{1}{2} J V\right)
$$

This is the generalization to higher dimensions of (3.20). Eq. (3.20) is re-obtained from (3.21) for $d_{A}=1$. In contrast to the one-dimensional case (3.20), $V$ and $\Sigma_{A}^{-1}$ are not proportional, in general.

There is a natural way obtain a semiclassical effective description with controllable phase space dimension of the spin model (3.1). For inhomogeneous states, which are not fully permutation invariant, but only invariant w.r.t. permutations within $d$ extensive subsets of the $N$ spins, the spin model (3.1) has an effective description in $2 d$ dimensional phase space, cf. Sec. 3.7. Moreover, the time evolved states after a quench in this model are approximately Gaussian in the large $N$ limit. Hence, in this situation, (3.21) can be applied to obtain the leading quadratic term of the entanglement Hamiltonian on $d$ dimensional state space.

\footnotetext{
${ }^{4}$ In general, the covariance matrix of a state $\rho_{A}$ is defined in $3.28 \mathrm{~b}$ below. For the special case when $\Psi(x) \propto$ $\exp (-x \Gamma x / 2)$ is a Gaussian wave function, the covariance of $\rho_{A}=\operatorname{Tr}_{B}|\psi\rangle_{A B}\langle\psi|$ is stated in Sec. 5.1 and appendix $\mathrm{F}$ of $[1]$.
} 


\section{Entanglement and magnetization variance in the transverse field Ising model}

Entanglement entropies. For large deviation states $\Psi\left(N_{+}\right) \asymp \exp \left[-N f\left(N_{+} / N\right)\right]$ with rate function $f$ and unimodal real part $\Re f$, the leading contribution of the $n$th bipartite Rényi entanglement cf. Eqs. (22), entropy in the large $N$ limit is, cf. table 3.1 .

(23), and appendix $\mathrm{G}$ in [1]

cf. Eq. (26) in $[1$

$$
S_{A}^{(n)}=\frac{1}{n-1} \log \left[(\lambda+1 / 2)^{n}-(\lambda-1 / 2)^{n}\right],
$$

where $\lambda$ is the $N$ independent symplectic eigenvalue of the covariance of the Wigner transform of the reduced density matrix $\rho_{A}$, and can be related to the $N$ independent squeezing parameter $\xi_{S}^{2}$. More precisely, $\xi_{S}^{2}$ is the ratio of the minimal to the maximal spin variance measured along directions perpendicular to the spin expectation value, and, cf. Appendix $\mathrm{H}$ of [1],

$$
\lambda=\sqrt{1+\alpha \beta\left(\xi_{S}+1 / \xi_{S}-2\right)} / 2 .
$$

Equations $(3.22)$ and $(3.23)$ are the main results concerning the bipartite entanglement entropy of the quantum spin model (3.1). It has been obtained in [1] in two different ways. First, as a direct consequence of the explicitly known spectrum of the entanglement Hamiltonian, cf. Secs. 5.2 and 5.3 of [1]. And second, by means of the replica trick, cf. appendix G of [1]. The equivalence of both approaches is explicitly demonstrated at the end of appendix $\mathrm{G}$.

The computation is facilitated by the effective description $(3.2)$ in which the large system limit translates into a semiclassical limit. We stress that despite using semiclassical techniques in the effective model, the computed entanglement entropy is still a pure quantum property of the spin model. Hence, the fact that the entanglement entropy scales as $\mathcal{O}\left(N^{0}\right)$ and obtains a non-zero limit does not contradict the semiclassical calculation, cf. Sec. 2.2. Still, the $N$-independence is not completely obvious.

In the remainder of this section we want to elaborate a little bit on the scaling of the entanglement entropy with $N$. To put this scaling of the entanglement entropy into perspective, we consider two limiting cases.

First, the entanglement of a coherent spin state

$$
\begin{aligned}
|\theta, \phi\rangle & =\sum_{N_{+}=0}^{N}\left(\begin{array}{c}
N \\
N_{+}
\end{array}\right)^{1 / 2}\left(\cos \frac{\theta}{2}\right)^{N_{+}}\left(e^{i \phi} \sin \frac{\theta}{2}\right)^{N_{-}}\left|N_{+}\right\rangle \\
& =\left(\cos \frac{\theta}{2}|\uparrow\rangle+e^{i \phi} \sin \frac{\theta}{2}|\downarrow\rangle\right)^{\otimes N}
\end{aligned}
$$

vanishes, because the state is a direct product state of the elementary spins. Second, consider the entanglement entropy of a Dicke state, cf. Eq. (2.2),

$$
\left|N_{+}\right\rangle=\left(\begin{array}{c}
N \\
N_{+}
\end{array}\right)^{1 / 2} P\left(|\uparrow\rangle^{\otimes N_{+}} \otimes|\downarrow\rangle^{\otimes N-N_{+}}\right)
$$

with fraction of up spins $N_{+} / N=n_{+}$. A direct computation (details are given in Appendix B) yields the $q$-th Rényi entanglement entropy

$$
S_{q}\left(N^{+}\right)=\frac{1}{q-1} \log \sqrt{q}+\frac{1}{2} \log \left[N 2 \pi \alpha \beta n^{+}\left(1-n^{+}\right)\right],
$$

in the large $N$ limit. The computation relies on a saddle point approximation, which is only valid if $N_{+} / N$ attains a limit $0<n_{+}<1$ as $N \rightarrow \infty$. The result (3.25) is not valid if $N_{+} / N$ or $1-N_{+} / N$ approaches zero in the limit $N \rightarrow \infty$. In particular, for the boundary cases of $N_{+}=0$ and $N_{+}=N$ the Dicke state $\left|N_{+}\right\rangle$is identical to the coherent state $|\theta=\pi\rangle$ and $|\theta=0\rangle$, respectively, and the entanglement entropy vanishes exactly. However, if $N_{+}$is away from the boundary, then, Eq. (3.25) 
states that the Dicke states are highly entangled. Away from the boundary the entanglement entropy of a Dicke state differs from the upper bound (3.19) of maximal entanglement only by an $N$-independent constant term.

Coming back to the state $|\Psi\rangle \asymp \sum_{N_{+}} \exp \left(-N f\left(N_{+} / N\right)\right)\left|N_{+}\right\rangle$. For rate functions $f$ with unimodal real part $\Re f$ and quadratic behavior close to its global minimum, the fraction of significant overlaps $\left\langle N_{+} \mid \Psi\right\rangle$ scales as $1 / \sqrt{N}$. In this sense, only a few Dicke states of similar $N_{+}$within a range of $\sqrt{N}$ give contributions to $|\Psi\rangle$. Yet, the scaling of the entanglement entropy of $|\Psi\rangle$ is very different from the almost maximal entanglement of a single Dicke state. These scaling observations are summarized schematically in Fig. 3.3 .

coherent state

large deviation state

$$
|\theta, \phi\rangle
$$$$
S=0
$$

Eq. 3.24

$$
|\Psi\rangle=\sum_{N_{+}} e^{-N f\left(N_{+} / N\right)}\left|N_{+}\right\rangle
$$$$
S=\mathcal{O}\left(N^{0}\right) \quad \ll
$$

Eq. (3.22)

$$
\begin{aligned}
& \begin{array}{c}
\text { Dicke state } \\
\left|N_{+}\right\rangle
\end{array} \\
& S=\mathcal{O}(\log N) \lesssim S=\log \alpha N+\mathcal{O}\left(N^{-1}\right)
\end{aligned}
$$

Eq. (3.25)

Eq. 3.19)

Figure 3.3: Scaling of (Rényi) entanglement entropies for different states with $N$ spins in the large $N$ limit. Second column: entanglement entropy of large deviation states $|\Psi\rangle$ with rate function $f$ saturates to an $N$-independent value. Even though a small fraction of $1 / \sqrt{N}$ of the overlaps $\left\langle N_{+} \mid \Psi\right\rangle$ give significant contributions to $|\Psi\rangle$, this scaling is very different from the entanglement entropy scaling $\mathcal{O}(\log N)$ of Dicke states $\left|N_{+}\right\rangle$(third column), which are close to being maximally entangled for $N_{+} / N \rightarrow n_{+}$with $0<n_{+}<1$ (fourth column).

\subsection{Symplectic squeezing and entanglement in higher dimensions *}

This section discusses the Rényi entanglement entropy of Gaussian states on a bipartite configuration space of arbitrary dimensions. One of the main results is Eq. (3.26). The fact that the entanglement entropy is a function of the symplectic eigenvalues of the reduced covariance matrix is emphasized. And the connection to (classical) symplectic squeezing is discussed. This result is not contained in the publication [1.

Motivation. It is known that the symplectic capacity is related to the uncertainty principle 132, 133. More precisely, the lower bound $c\left(\mathcal{W}_{\Sigma}\right) \geq \pi \hbar$ on the symplectic capacity of the Wigner ellipsoid $\mathcal{W}_{\Sigma}=\left\{z: \frac{1}{2} z \Sigma^{-1} z \leq 1\right\}$ associated to the covarianc ${ }^{5} \Sigma$ of a state $\rho$, implies the (strong) uncertainty principle. Moreover, the connection between uncertainty and entanglement in (collective) spin systems is established through spin squeezing. Hence, it is natural to expect a direct connection between entanglement and symplectic capacity for collective spins. In fact, for Gaussian densities on one-dimensional configuration space, $n=1$, the Rényi entropies are functions of the symplectic capacity, see (3.22). Now, the one-dimensional case, $n=1$, is special in the

${ }^{5}$ Here, the covariance $\Sigma$ is defined for any mixed state $\rho$ (not necessarily Gaussian) by

$$
\Sigma=\operatorname{Tr}\left[\rho\left(\begin{array}{cc}
\widetilde{x}^{2} & (\widetilde{x} \widetilde{p}+\widetilde{p} \widetilde{x}) / 2 \\
(\widetilde{x} \widetilde{p}+\widetilde{p} \widetilde{x}) / 2 & \widetilde{p}^{2}
\end{array}\right)\right],
$$

where $\widetilde{x}=x-\operatorname{Tr} \rho x$, and $\widetilde{p}=p-\operatorname{Tr} \rho p$, also see the discussion around 3.28. 


\section{Entanglement and magnetization variance in the transverse field Ising model}

sense that the Wigner ellipsoid is two-dimensional, its symplectic capacity is simply the area, and there is only one symplectic eigenvalue of the two by two covariance matrix. It is important to investigate how the calculation generalizes to $n>1$, and whether the entanglement entropy is still a function of the symplectic capacity or other quantities related to the symplectic spectrum, such as the volume of the Wigner ellipsoid. The answer is given by Eq. (3.26), its derivation is as follows.

All Rényi entropies of a Gaussian density matrix $\rho$ are symplectic invariants, and depend only on the symplectic spectrum of the Wigner function's covariance matrix. To see this, change variables $z \mapsto S z$, in

$$
\begin{aligned}
S_{\alpha}=\frac{1}{1-\alpha} \log \operatorname{Tr} \rho^{\alpha} & =\frac{1}{1-\alpha} \log \int W_{\rho}^{* \alpha}(z) \frac{d^{2 n} z}{(2 \pi)^{n}} \\
& =\frac{1}{1-\alpha} \log \int W_{\rho}^{* \alpha}(S z) \frac{d^{2 n} z}{|\operatorname{det} S|(2 \pi)^{n}},
\end{aligned}
$$

where $W^{* \alpha}$ denotes the Moyal star product of $\alpha$ factors of $W(z)$. According to Williamson's theorem, we choose $S$ to be a symplectic matrix (in particular, $\operatorname{det} S=1$ ) that diagonalizes $\Sigma^{-1}$, such that

$$
W_{\rho}(S z) \propto \exp \left[-\frac{1}{2} z S^{T} \Sigma^{-1} S z\right]=\exp \left[-\frac{1}{2} z\left(\begin{array}{cc}
\Lambda^{-1} & 0 \\
0 & \Lambda^{-1}
\end{array}\right) z\right]
$$

where the eigenvalues of $\Lambda=\operatorname{diag}\left(\lambda_{1}, \cdots \lambda_{n}\right)$ are the symplectic eigenvalues of $\Sigma$. Hence, the entanglement entropy of $\rho$ depends on $W_{\rho}$ only through the symplectic spectrum of the covariance. In these transformed coordinates (which we denote by the same symbol, $z$ ), the Wigner function factorizes, $W_{\rho}(z)=\prod_{j=1}^{n} W_{j}\left(z_{j}\right)$, where $z_{j}=\left(x_{j}, p_{j}\right)$, and

$$
W_{j}\left(z_{j}\right) \propto \exp \left[-\frac{1}{2} z_{j} \lambda_{j}^{-1} z_{j}\right] .
$$

Thus ${ }^{6}, W_{\rho}^{* \alpha}(z)=\prod_{j=1}^{n} W_{j}^{* \alpha}\left(z_{j}\right)$. One can compute the Moyal-star monomials of the two dimensional Gaussians $W_{j}$ explicitly, see e.g. 134, 135. Instead, we employ the $n=1$ result (3.22),

$$
\begin{aligned}
S_{\alpha} & =\frac{1}{1-\alpha} \log \prod_{j=1}^{n} \int W_{j}^{* \alpha}(z) \frac{d^{2} z}{2 \pi} \\
& \stackrel{3.22}{-} \frac{1}{\alpha-1} \sum_{j=1}^{n} \log \left[\left(\lambda_{j}+1 / 2\right)^{\alpha}-\left(\lambda_{j}-1 / 2\right)^{\alpha}\right] \\
& =\sum_{\lambda \in \operatorname{Spec}_{J}(\Sigma)} S_{\alpha}(\lambda),
\end{aligned}
$$

where

$$
S_{\alpha}(\lambda)=\frac{1}{\alpha-1} \log \left[(\lambda+1 / 2)^{\alpha}-(\lambda-1 / 2)^{\alpha}\right],
$$

and $\operatorname{Spec}_{J}(\Sigma)=\left\{\lambda_{1}, \cdots \lambda_{n}\right\}$ denotes the symplectic spectrum of $\Sigma$. In words, the $\alpha$ Rényi entropy of the Gaussian density matrix $\rho$ on $n$-dimensional configuration space with covariance matrix $\Sigma$, is the sum of $n$ terms of the form $S_{\alpha}(\lambda)$, where the summation ranges over the symplectic

\footnotetext{
${ }^{6}$ As a consequence of $(f * g)(z)=f(z) \exp \left(-\frac{i \hbar}{2} \sum_{j} \Lambda_{j}\right) g(z)=f(z) \prod_{j} \exp \left(-\frac{i \hbar}{2} \Lambda_{j}\right) g(z)$, where $\Lambda_{j}=\overleftarrow{\partial}_{p_{j}} \vec{\partial}_{x_{j}}-$ $\overleftarrow{\partial}_{x_{j}} \vec{\partial}_{p_{j}}$
} 


\subsection{Symplectic squeezing and entanglement in higher dimensions *}

eigenvalues $\lambda$ (counting multiplicity) of $\Sigma$. Each term $S_{\alpha}(\lambda)$ is the $\alpha$ Rényi entropy of a Gaussian density matrix on one-dimensional configuration space, whose covariance matrix has symplectic eigenvalue $\lambda$. A few explicit expressions for special Rényi entropies are summarized in table 3.1 .

Remarks. - As $\Sigma$ is the covariance matrix of the Wigner function of a positive, normalized, Hermitian density matrix, all its symplectic eigenvalues are bounded from below by one half (KLM criterion, see chapter 13 in [133], and Corollary 9.36 in [132]). Hence, the term $\left(\lambda_{j}-1 / 2\right)$ is non-negative.

- As $S_{\alpha}(\lambda)$ is non-decreasing as a function of $\lambda$, the symplectic capacity $c=2 \pi \min \operatorname{Spec}_{J}(\Sigma)$ of the Wigner ellipsoid associated to $\rho$ sets a lower bound on the entanglement entropy, $S_{\alpha} \geq \frac{n}{\alpha-1} \log \left[\left(\frac{c}{2 \pi}+\frac{1}{2}\right)^{\alpha}-\left(\frac{c}{2 \pi}-\frac{1}{2}\right)^{\alpha}\right]$. In particular, the entanglement entropy vanishes if, and only if, all symplectic eigenvalues are one half.

- For a rank-one projection, i.e. a pure state, all symplectic eigenvalues of $\Sigma$ are equal to one half, and all Rényi entropies vanish.

- As a consistency check, $\operatorname{Tr} \rho=\lim _{\alpha \rightarrow 1} \exp \left[(1-\alpha) S_{\alpha}\right]=\prod_{j}\left[\left(\lambda_{j}+1 / 2\right)-\left(\lambda_{j}-1 / 2\right)\right]=1$.

- The von Neumann entropy, $\alpha \rightarrow 1$, is $S_{1}=\sum_{j=1}^{n}\left(\lambda_{j}+1 / 2\right) \log \left(\lambda_{j}+1 / 2\right)-\left(\lambda_{j}-1 / 2\right) \log \left(\lambda_{j}-\right.$ $1 / 2)$. This result has also been obtained in the calculation of the capacity of a Gaussian quantum channel in [136].

- The collision entropy (logarithm of purity), $\alpha \rightarrow 2$, is $S_{2}=\sum_{j} \log \left(2 \lambda_{j}\right)=\log \operatorname{vol}\left(\mathcal{W}_{\Sigma}\right)-$ $\log \operatorname{vol}\left(B_{2 n}(1)\right)$, equals the logarithm of the fraction of the volume $\operatorname{vol}\left(\mathcal{W}_{\Sigma}\right)=2^{n} \operatorname{vol}\left(B_{2 n}(1)\right) \prod_{j} \lambda_{j}$ of the the Wigner ellipsoid $\mathcal{W}_{\Sigma}=\left\{z: \frac{1}{2} z \Sigma^{-1} z \leq 1\right\}$ to the volume of the $2 n$ dimensional unit ball $B_{2 n}(1)$.

- The min entropy, $\alpha \rightarrow \infty$, is $S_{\infty}=\sum_{j=1}^{n} \log \left(\lambda_{j}+1 / 2\right)$.

\begin{tabular}{c|cccc} 
& von Neumann $S_{1}$ & Collision $S_{2}$ & Min $S_{\infty}$ & Rényi $S_{n}$ \\
\hline$\lambda=\frac{1}{2} \frac{1+\xi}{1-\xi}$ & $\begin{array}{c}\left(\lambda+\frac{1}{2}\right) \log \left(\lambda+\frac{1}{2}\right)- \\
\left(\lambda-\frac{1}{2}\right) \log \left(\lambda-\frac{1}{2}\right)\end{array}$ & $\log 2 \lambda$ & $\log (\lambda+1 / 2)$ & $\begin{array}{c}\frac{1}{n-1} \log \left[\left(\lambda+\frac{1}{2}\right)^{n}-\right. \\
\left.\left(\lambda-\frac{1}{2}\right)^{n}\right]\end{array}$ \\
\hline $\begin{array}{c}\omega= \\
2 \operatorname{arctanh}[1 /(2 \lambda)]\end{array}$ & $\begin{array}{c}-\log \left(1-e^{-\omega}\right)+ \\
\frac{\omega e^{-\omega}}{1-e^{-\omega}}\end{array}$ & $\log \frac{1+e^{-\omega}}{1-e^{-\omega}}$ & $-\log \left(1-e^{-\omega}\right)$ & $\frac{1}{1-n} \log \frac{\left(1-e^{-\omega}\right)^{n}}{1-e^{-n \omega}}$ \\
\hline$\xi=\exp (-\omega)$ & $H_{2}(\xi) /(1-\xi)$ & $\log \frac{1+\xi}{1-\xi}$ & $-\log (1-\xi)$ & $\frac{1}{1-n} \log \frac{(1-\xi)^{n}}{1-\xi^{n}}$
\end{tabular}

Table 3.1: Summary of the general Rényi entanglement entropy (last column), and important special cases, for a Gaussian reduced density matrix $\rho_{A}$ on a one-dimensional reduced state space. Different explicit expressions are given: (first row) as a function of the symplectic eigenvalue $\lambda$ of the reduced covariance $\Sigma_{A}$, (second row) as a function of the angular frequency $\omega$ of the quadratic entanglement Hamiltonian $H_{E}=-\log \rho_{A}$, cf. Sec. 3.3. and, (third row) as a function of $\xi$, which plays an important role in the replica trick, and in the $j$ th eigenvalue $(1-\xi) \xi^{j}$ of $\rho_{A}$, cf. appendix G in [1]. More generally, the entropy of a Gaussian reduced density matrix on higher dimensional state space is given by the sum of the one-dimensional result over the symplectic spectrum of $\Sigma_{A}$, cf. (3.26a). 
Symplectic squeezing and entanglement. Consider the situation of a pure Gaussian state $\psi^{A B}$ in $\mathcal{H}=\mathcal{H}_{A} \otimes \mathcal{H}_{B}=L^{2}\left(\mathbb{R}^{n_{A}}\right) \otimes L^{2}\left(\mathbb{R}^{n_{B}}\right)=L^{2}\left(\mathbb{R}^{n}\right)$, whose Wigner function $W^{A B}$ is Gaussian with covariance matrix $\Sigma^{A B}$. If the state is time evolved according to a (time-dependent) quadratic Hamiltonian (as in the nearby orbit approximation), the state remains Gaussian and the covariance matrix evolves as $\Sigma_{t}^{A B}=S_{t} \Sigma^{A B} S_{t}^{T}$, where $S_{t} \in \operatorname{Sp}(2 n)$. In general, the Wigner ellipsoid $\mathcal{W}_{t}^{A B}$ associated to $\Sigma_{t}^{A B}$ will be squeezed, and sheared, and not merely rotated, because $S_{t}$ does not need to be orthogonal (symplectic transformations that are not orthogonal, are sometimes called active transformations, while orthogonal symplectic transformation are called passive [137]). Hence, the spectrum of $\Sigma_{t}^{A B}$ is not time invariant. However, the symplectic spectrum of $\Sigma_{t}^{A B}$ is time invariant, in particular, all symplectic eigenvalues of $\Sigma_{t}^{A B}$ are equal to one half. Equivalently, the state $\psi^{A B}$ remains pure under unitary time evolution.

\begin{tabular}{|c|c|c|c|c|c|}
\hline $\begin{array}{c}\text { tensor product } \\
L^{2}\left(\mathbb{R}^{n}\right)= \\
L^{2}\left(\mathbb{R}^{n_{A}}\right) \otimes L^{2}\left(\mathbb{R}^{n_{B}}\right)\end{array}$ & $\begin{array}{c}\text { Schrödinger } \\
\text { Eq. }\end{array}$ & $\begin{array}{c}\text { flow of } \rho^{A B} \\
\text { unitary }\end{array}$ & $\begin{array}{c}\text { flow of } \rho^{A} \text { not } \\
\text { unitary }\end{array}$ & $\rho^{A B}$ pure & $\begin{array}{l}\rho^{A} \text { mixed } \\
\quad\left(\rho^{A B}\right. \\
\text { entangled })\end{array}$ \\
\hline $\begin{array}{c}\text { direct product } \\
\mathbb{R}^{2 n}=\mathbb{R}^{2 n_{A}} \oplus \mathbb{R}^{2 n_{B}}\end{array}$ & $\begin{array}{l}\text { Hamilton } \\
\text { Eq. }\end{array}$ & $\begin{array}{l}\text { flow of } W^{A B} \\
\text { symplectic }\end{array}$ & $\begin{array}{c}\text { flow of } W^{A} \\
\text { not symplectic }\end{array}$ & $\begin{array}{l}\operatorname{Spec}_{J}\left(\Sigma^{A B}\right) \\
\quad=\{1 / 2\}\end{array}$ & $\begin{array}{c}\operatorname{Spec}_{J}\left(\Sigma^{A}\right) \neq \\
\{1 / 2\}\end{array}$ \\
\hline
\end{tabular}

Table 3.2: Comparison between the unitary quantum dynamics (top row), and the symplectic classical Liouville dynamics (bottom row) of a bipartite Gaussian pure initial state $\psi^{A B}$ with corresponding Wigner function $W^{A B}$. For a quadratic (time-dependent) Hamiltonian the approximation of the quantum dynamics by the Liouville dynamics is exact. The entropy of the reduced state $\rho^{A}$ is a function of the symplectic eigenvalues of the covariance $\Sigma^{A}$ (last column) of the marginal Wigner function $W^{A}$, cf. (3.26). In general, the dynamics of $\rho^{A}$ and $W^{A}$ is not unitary and symplectic, respectively, (fourth column). As a consequence, the symplectic spectrum of $\Sigma^{A}(t)$ and the eigenvalues of $\rho^{A}(t)$ are generally time-dependent, such that the entanglement entropy of $\rho^{A B}(t)$ is time-dependent.

Now, a description of subsystem $A$, discarding system $B$, is obtained by taking the partial trace over subsystem $B$ in $\rho^{A}=\operatorname{Tr}_{B}\left|\psi^{A B}\right\rangle\left\langle\psi^{A B}\right|$, and, equivalently, marginalizing the Wigner function $W^{A}=\int_{B} W^{A B}$, i.e. projecting the classical (quasi-probability) density onto the symplectic subspace of subsystem $A$. All marginals of a Gaussian remain Gaussian functions of lower dimension, and the covariance matrix $\Sigma^{A}=P_{A} \Sigma^{A B} P_{A}$ of the marginal Wigner function $W^{A}$ is obtained by projecting $\Sigma^{A B}$ onto the symplectic subspace of subsystem $A$ with $P_{A}:\left(x_{A}, x_{B}, p_{A}, p_{B}\right) \mapsto\left(x_{A}, p_{A}\right)$. (In coordinates, all rows and columns of $\Sigma^{A B}$ belonging to position and momenta of subsystem $B$ are deleted.) There are two important consequences of this projection. First, in the same way as the evolution of $\rho^{A}$ does not need to be unitary (unless the Hamiltonian does not couple subsystems $A$ and $B$ ), the flow of the Wigner function $W^{A}$ does not need to be symplectic. In particular, $\Sigma_{t}^{A}=S_{t}^{A} \Sigma^{A} S_{t}^{A T}$ evolves according to a transformation $S_{t}^{A}$, which is not necessarily symplectic. Hence, the symplectic spectrum of $\Sigma_{t}^{A}$ is not time invariant. Together with (3.26), this implies that the entanglement entropy is not necessarily time invariant, cf. table 3.2 . Second, the symplectic spectrum of $\Sigma^{A}$ is bounded from below by the symplectic spectrum of $\Sigma^{A B}$ in the following sense. Let $\left(\lambda_{1}^{A}, \cdots \lambda_{n_{A}}^{A}\right)$, and $\left(\lambda_{1}^{A B}, \cdots \lambda_{n}^{A B}\right)$ be the sequence of non-decreasingly ordered symplectic eigenvalues (counting multiplicity) of $\Sigma^{A}$, and $\Sigma^{A B}$, respectively. Then $\lambda_{j}^{A B} \leq \lambda_{j}^{A}$ for all $j=1, \cdots n_{A}$. This is a consequence of the symplectic non-squeezing theorem [138 140]. Intu- 


\subsection{Symplectic squeezing and entanglement in higher dimensions *}

itively, to obtain the smallest symplectic eigenvalue $\lambda_{1}^{A}$ of $\Sigma^{A}$, i.e. the symplectic capacity $c^{A}$ of the associated Wigner ellipsoid $\mathcal{W}^{A}$ divided by $2 \pi$, one deforms the Wigner ellipsoid by the optimal symplectic transformation to make it fit into the smallest possible cylinder based on a symplectic plane. The radius of the optimal cylinder is $\sqrt{2 \lambda_{1}^{A}}$, and its area $c^{A}=2 \pi \lambda_{1}^{A}$ is the capacity (see chapter 5 in 133 ). Since the symplectic capacity $c^{A B}=\pi$ (minimal uncertainty in units in which $\hbar=1$ ) of $\mathcal{W}^{A B}$ is constant, one can always find a symplectic transformation to squeeze the Wigner ellipsoid $\mathcal{W}^{A B}$ into a cylinder of radius $\sqrt{c^{A B} / \pi}=1$ (equivalently, $\lambda_{1}^{A B}=1 / 2$ ). However, the set of symplectic transformations on the symplectic subspace $A$ is smaller than the set of all symplectic transformations of the ambient space. Hence, in general, the optimal symplectic transformations that fits $\mathcal{W}^{A B}$ into a cylinder of radius one, may not be a valid symplectic transformation on subspace $A$. Then, if the Wigner ellipsoid $\mathcal{W}^{A}$ cannot be fitted into a cylinder of radius $\sqrt{c^{A B} / \pi}$, the symplectic capacity $c^{A}$ of $\mathcal{W}^{A}$ is larger than the symplectic capacity $c^{A B}$ of $\mathcal{W}^{A B}$ (but never smaller), and $\lambda_{1}^{A B} \leq \lambda_{1}^{A}$. Similarly, one infers $\lambda_{j}^{A B} \leq \lambda_{j}^{A}$ for $j=1 \cdots n_{A}$. A more precise formulation is given by the symplectic interlacing theorem below. This gives a qualitative geometric interpretation of the growth of the entanglement entropy. The entanglement entropy of subsystem $A$ with $B$ becomes large when the Wigner ellipsoid $\mathcal{W}^{A B}$ is symplectically deformed such that its orthogonal projection $\mathcal{W}^{A}$ onto A cannot be squeezed into a cylinder of small radius. In particular, for $n_{A}=1$ the entanglement entropy $S_{\alpha}(A)$ is a monotonic function of the symplectic capacity of the Wigner ellipsoid $\mathcal{W}^{A}$.

Interlacing theorems. Mathematically, this leads to the following question. Given a positive, symmetric $2 n$ by $2 n$ matrix $\Sigma^{A B}$ with symplectic eigenvalues $\lambda_{1}^{A B} \leq \cdots \leq \lambda_{n}^{A B}$, how do the symplectic eigenvalues change upon orthogonal projection onto a symplectic subspace of dimension $2 n_{A}$ ? That is, how are the symplectic eigenvalues $\lambda_{1}^{A} \leq \cdots \leq \lambda_{n_{A}}^{A}$ of $\Sigma^{A}=P_{A} \Sigma^{A B} P_{A}$ constrained by the eigenvalues $\lambda_{j}^{A B}$ ? Physically, this problem is known as the Gaussian marginal problem and has been investigated in [137, 141]. The analogous problem for the the (orthogonal) spectrum, is solved by the min-max theorem (a refinement of the Rayleigh-Ritz variational principle), and the solution is known as the Cauchy interlacing theorem, see appendix. The symplectic analog of the Cauchy interlacing theorem states [137],

$$
\begin{aligned}
\lambda_{j}^{A B} & \leq \lambda_{j}^{A}, \text { for all } j=1 \cdots n_{A}, \\
\text { and } \lambda_{j}^{A B} & \leq \lambda_{j}^{A} \leq \lambda_{j+2 n_{B}}^{A B} \text {, for } j=1 \cdots 2 n_{A}-n,
\end{aligned}
$$

where $n_{B}=n-n_{A}$ is the codimension of subsystem $A$. The case $n_{A}=1$ is discussed in [141].

Example (Coherent state). The Wigner function of a coherent state in $\mathcal{H}^{A} \otimes \mathcal{H}^{B}$ has uniform covariance, i.e. $\Sigma^{A B}=\mathbf{1}_{2 n \times 2 n} / 2$, and both, the symplectic and orthogonal spectrum are equal to $\operatorname{Spec}(\Sigma)=\operatorname{Spec}_{J}(\Sigma)=\{1 / 2\}$. The Wigner ellipsoid is a ball of radius one half, and the orthogonal projection on any subspace yields a ball of the same radius in lower dimensions. Hence, $\Sigma^{A}=\mathbf{1}_{2 n_{A} \times 2 n_{A}} / 2$, the orthogonal and symplectic spectrum of $\Sigma^{A}$ is equal to $\{1 / 2\}$ and the state is not entangled.

As time evolves, the covariance changes according to $\Sigma_{t}^{A B}=S_{t} \Sigma^{A B} S_{t}^{T}=S_{t} S_{t}^{T} / 2$ with $S_{t} \in$ $\operatorname{Sp}(2 n)$. For non orthogonal (i.e. active) $S_{t}$, the Wigner ellipsoid is no longer a ball and the (orthogonal) spectrum of $\Sigma_{t}^{A B}$ changes, while the symplectic spectrum remains $\{1 / 2\}$. Projecting the Wigner ellipsoid onto the symplectic subspace of system $A$ via $P_{A}$ corresponds to projecting $\Sigma_{t}^{A}=P_{A} \Sigma_{t}^{A B} P_{A}$. The orthogonal spectrum of $\Sigma_{t}^{A}$ is related to the spectrum of $\Sigma_{t}^{A B}$ by Cauchy's interlacing problem. In general, the symplectic spectrum of $\Sigma_{t}^{A}$ also changes, obeying the constraint (3.27), and the evolved state becomes entangled. 


\subsection{Interlude: bound on entanglement of non-Gaussian states $*$}

The main result of this section is Eq. (3.28). It uses the results of the previous section to bound the von Neumann entropy of a generic density matrix. This is a new unpublished result, not contained in [1].

The result (3.26) gives the entanglement entropy for Gaussian states. However, many states of interest are not close to Gaussians. Even if one initially prepares a Gaussian state, it will generically deviate from the Gaussian type in the course of time. What can one infer from (3.26) about the entanglement entropy of non-Gaussian density matrices? Gaussian density matrices are special, and the calculation leading to 3.26 does not generalize directly to more general density matrices. Notwithstanding, (3.26) can be used to give the upper bound

$$
\begin{aligned}
S_{\mathrm{vN}}(\rho)=-\operatorname{Tr} \rho \log \rho & \leq \sum_{\lambda \in \operatorname{Spec}_{J}(\Sigma)} S_{\alpha}(\lambda) \\
& =\sum_{j=1}^{n}\left(\lambda_{j}+1 / 2\right) \log \left(\lambda_{j}+1 / 2\right)-\left(\lambda_{j}-1 / 2\right) \log \left(\lambda_{j}-1 / 2\right)
\end{aligned}
$$

on the von Neumann entropy of a generic (possibly non-Gaussian) density matrix $\rho$ in terms of the symplectic eigenvalues $\operatorname{Spec}_{J}(\Sigma)=\left(\lambda_{1}, \cdots \lambda_{n}\right)$ of its $2 n$ by $2 n$ covariance matrix

$$
\Sigma=\operatorname{Tr}\left[\rho\left(\begin{array}{cc}
\widetilde{x}^{2} & (\widetilde{x} \widetilde{p}+\widetilde{p} \widetilde{x}) / 2 \\
(\widetilde{x} \widetilde{p}+\widetilde{p} \widetilde{x}) / 2 & \widetilde{p}^{2}
\end{array}\right)\right],
$$

where $\widetilde{x}=x-\operatorname{Tr} \rho x$, and $\widetilde{p}=p-\operatorname{Tr} \rho p$.

The classical analog of $(3.28)$ is the textbook fact that the Gaussian probability density $\rho_{\text {Gauss }}(x) \propto$ $e^{-x C^{-1} x / 2}$ maximize the Shannon entropy among all classical probability densities with (co)variance matrix $C$ 142. Its proof relies on the positivity of the relative entropy. In what follows we give a proof of the quantum analog (3.28). Essentially, the idea of the classical proof translates to the phase space formulation of the quantum situation. An important difference to the classical case is the use of the star-logarithm instead of the regular logarithm function of the classical Shannon entropy.

Proof of (3.28). Let $\rho_{\text {Gauss }}$ be the Gaussian density matrix with the same covariance matrix $\Sigma$ as $\rho$, cf. $3.28 \mathrm{~b}$ ). Just as the classical relative entropy, the quantum relative entropy $D(\rho \| \sigma)=$ $\operatorname{Tr} \rho \log \rho-\operatorname{Tr} \rho \log \sigma \geq 0$ between any two density matrices $\rho$ and $\sigma$ is non-negative (Klein's inequality) and vanishes if and only if $\rho=\sigma$, cf. chapter 11 of [25]. In particular,

$$
D\left(\rho \| \rho_{\text {Gauss }}\right) \geq 0
$$

and in terms of the Wigner functions $W_{\rho}$ and $W_{\text {Gauss }}(z) \propto \exp \left(-\frac{1}{2} z \Sigma^{-1} z\right)$ of $\rho$ and $\rho_{\text {Gauss }}$, respectively,

$$
D\left(\rho \| \rho_{\text {Gauss }}\right)=\int_{\mathbb{R}^{2 n}} \frac{d z}{(2 \pi)^{n}} W_{\rho} \log ^{*} W_{\rho}-\int_{\mathbb{R}^{2 n}} \frac{d z}{(2 \pi)^{n}} W_{\rho} \log ^{*} W_{\text {Gauss }} \geq 0,
$$

Here, $\log ^{*}$ denotes the star-logarithm, the inverse function of the star-exponential [134,143]. The occurrence of the star-logarithm instead of the regular logarithm is crucial. If one replaces the starlogarithm in $-\int W_{\rho} \log ^{*} W_{\rho}$ by the regular logarithm, one gets the Shannon entropy of the Wigner quasi-probability distribution, related to Wehrl's entropy [144]. The von-Neumann entropy of a density matrix $\rho$ is not simply the Shannon entropy of its Wigner quasi-probability distribution. In 
general, the star-logarithm of a generic phase space function is difficult to compute, as it requires Moyal-star monomials of arbitrary powers. However, the star-logarithm of the Gaussian density $W_{\text {Gauss }}$ can be worked out explicitly, see appendix A,

$$
\log ^{*} \exp \left(-\frac{1}{2} z \Sigma^{-1} z\right)=-\frac{1}{2} z V z-\log \mathcal{C}
$$

where the matrices $\Sigma$ and $V$ are related by $\Sigma^{-1}=-2 J|J V| \tan \left(\frac{1}{2} J V\right)$, and $\mathcal{C}^{-1}=\sqrt{\operatorname{det} \cos J V / 2}$. The precise form of the right hand side is not important. The crucial thing is that $\log ^{*} W_{\text {Gauss }}$ is a quadratic form in the phase space coordinate $z$. Because $\log ^{*} W_{\text {Gauss }}$ is integrated against $W_{\rho}$ in the second term of (3.29b), and the (co)variance of $W_{\rho}$ and $W_{\text {Gauss }}$ agree by assumption, one may replace the second integral in $3.29 \mathrm{~b}$ by

$$
S_{\mathrm{vN}}\left(\rho_{\text {Gauss }}\right)=-\int_{\mathbb{R}^{2 n}} \frac{d z}{(2 \pi)^{n}} W_{\text {Gauss }} \log ^{*} W_{\text {Gauss }} .
$$

This yields $S_{\mathrm{vN}}(\rho) \leq S_{\mathrm{vN}}\left(\rho_{\text {Gauss }}\right)$, and is equivalent to the claim (3.28).

\subsection{Multipartite entanglement $*$}

This section sketches how the computation of the bipartite entanglement entropy in the fully connected spin model (3.1) is generalized to quadripartitions. An example for a quadripartite entanglement measure is the tripartite information $I_{3}$. The tripartite information is also used to cf. Sec. 5 in 1 measure how information delocalizes in unitary quantum channels 145, 146

The main result of this section is Eq. (3.35). It shows that the dynamics of the tripartite information after a quench in the fully connected spin model does not yield more information than the dynamics of the bipartite entanglement entropy.

Quadripartite entanglement for time evolved pure states. Let $\left|\Psi_{t}\right\rangle=e^{-i H t}\left|\Psi_{0}\right\rangle$ be the time evolved state after a quantum quench in $\Gamma$ of (3.1). The dynamics takes place in the Dicke subspace $\mathcal{D}_{N}$ of $N$ spins. The bipartite entanglement dynamics of $\left|\Psi_{t}\right\rangle$ was investigated in [1]. How does this calculation translate to multipartite entanglement measures?

One quadripartite entanglement measure of pure states is the tripartite information

$$
\begin{aligned}
I_{3}(A: C: D) & =I(A: C)+I(A: D)-I(A: C D) \\
& =S(A)+S(C)+S(D)-S(A C)-S(A D)-S(C D)+S(A C D)
\end{aligned}
$$

For the quadripartition $\mathcal{H}_{N}=\mathcal{H}_{A} \otimes \mathcal{H}_{B} \otimes \mathcal{H}_{C} \otimes \mathcal{H}_{D}$ of $N$ spins into four disjoint subsets of $A$, $B, C$ and $D$ spins with $A+B+C+D=N$, any state $|\Psi\rangle=\sum_{N_{+}} \Psi\left(N_{+}\right)\left|N_{+}\right\rangle$in $\mathcal{D}_{N}$ can be 
expanded ${ }^{7}$ in $\mathcal{D}_{A B C D}:=\mathcal{D}_{A} \otimes \mathcal{D}_{B} \otimes \mathcal{D}_{C} \otimes \mathcal{D}_{D} \supset \mathcal{D}_{N}$ as

$$
\begin{aligned}
|\Psi\rangle=\sum_{A_{+}, B_{+}, C_{+}, D_{+}} \Psi\left(A_{+}+B_{+}+C_{+}+D_{+}\right) \\
\qquad \sqrt{\left(\begin{array}{c}
A \\
A_{+}
\end{array}\right)\left(\begin{array}{c}
B \\
B_{+}
\end{array}\right)\left(\begin{array}{c}
C \\
C_{+}
\end{array}\right)\left(\begin{array}{c}
D \\
D_{+}
\end{array}\right) /\left(\begin{array}{c}
N \\
A_{+}+B_{+}+C_{+}+D_{+}
\end{array}\right)}\left|A_{+}, B_{+}, C_{+}, D_{+}\right\rangle,
\end{aligned}
$$

compare (3.18). The expansion coefficient in front of $\left|A_{+}, B_{+}, C_{+}, D_{+}\right\rangle$is denoted by $\Psi\left(A_{+}, B_{+}, C_{+}, D_{+}\right)$. The combinatorial factor

$$
\left(\begin{array}{c}
A \\
A_{+}
\end{array}\right)\left(\begin{array}{c}
B \\
B_{+}
\end{array}\right)\left(\begin{array}{c}
C \\
C_{+}
\end{array}\right)\left(\begin{array}{c}
D \\
D_{+}
\end{array}\right) /\left(\begin{array}{c}
N \\
A_{+}+B_{+}+C_{+}+D_{+}
\end{array}\right) \asymp \exp \left[-N S_{\alpha \beta \gamma \delta}(a, b, c, d)\right]
$$

takes into account the fact that there are more ways to permute $N_{+}$up-spins among $N$ spins than to independently permute the up-spins within the disjoint subsets of spins without inter-subset permutations.

Let $f_{t}\left(n_{+}\right)$be the rate function of $\left|\Psi_{t}\right\rangle \in \mathcal{D}_{N}$, i.e. $\Psi_{t}\left(n_{+} N\right) \asymp \exp \left[-N f_{t}\left(n_{+}\right)\right]$. The minimum of $\Re f_{t}\left(n_{+}\right)$at $n_{+}=n_{t}$ evolves according to Hamilton's equation of motion for the effective Hamiltonian $H_{\text {eff }}$, cf. Sec. 3.2. We denote the curvature of $f_{t}$ at the minimum by $f_{t}^{\prime \prime}\left(n_{t}\right)=: f_{2}=$ cf. Eq. (16) in [1] $g_{2}-i \theta_{2}$, cf. Eq. (3.5). From (3.32) it follows that the rate function of $\Psi_{t}\left(A_{+}, B_{+}, C_{+}, D_{+}\right) \asymp$ $\exp \left[-N f_{t}^{(4)}(a, b, c, d)\right]$ is

$$
f_{t}^{(4)}(a, b, c, d)=f_{t}(\alpha a+\beta b+\gamma c+\delta d)+\frac{1}{2} S_{\alpha \beta \gamma \delta}(a, b, c, d)
$$

where $S_{\alpha \beta \gamma \delta}(a, b, c, d)=H_{2}(\alpha a+\beta b+\gamma c+\delta d)-\alpha H_{2}(a)-\beta H_{2}(b)-\gamma H_{2}(c)-\delta H_{2}(d)$ is the entropic rate function of the combinatorial factor (3.33). $S_{\alpha \beta \gamma \delta}(a, b, c, d)$ depends on the relative subsystem sizes $\alpha=A / N, \beta=B / N, \gamma=C / N, \delta=D / N$, and is a function of the fraction of up spins $a=A_{+} / A, b=B_{+} / B, c=C_{+} / C, d=D_{+} / D$ in the respective subsystems. Convexity (and $\alpha+\beta+\gamma+\delta=1$ ) of the binary Shannon entropy guarantees that $S_{\alpha \beta \gamma \delta}$ is non-negative and vanishes if, and only if, $a=b=c=d$. Hence, the minimum of $\Re f_{t}$ at $n_{t}$ is inherited to the minimum of $\Re f_{t}^{(4)}$ at $(a, b, c, d)=\left(n_{t}, n_{t}, n_{t}, n_{t}\right)=: \mathbf{x}^{*}$.

Expanding $f_{t}^{(4)}(a, b, c, d)$ to second order around its minimum at $\mathbf{x}^{*}$, yields a Gaussian approxi-

cf. the discussion of Eq. (17) in 1] mation $\Psi_{t}^{A B C D}(\mathbf{x}) \propto \exp \left[-\frac{N}{2}\left(\mathbf{x}-\mathbf{x}^{*}\right) \Gamma^{(4)}\left(\mathbf{x}-\mathbf{x}^{*}\right)\right]$ with four by four inverse covariance

$$
\Gamma^{(4)}=\left.\nabla^{2} f^{(4)}(\mathbf{x})\right|_{\mathbf{x}=\mathbf{x}^{*}}
$$

being the Hessian matrix of the quadripartite rate function. Real and imaginary part of $\Gamma^{(4)}$ are

${ }^{7}$ The fact that $\mathcal{D}_{A B C D}$ is larger than the Dicke space $\mathcal{D}_{N}$ can be seen by counting dimensions. Intuitively, $\mathcal{D}_{A B C D}$ contains states that are permutation invariant among their elementary spins within each subgroup $A, B, C$ and $D$. However, these states do not need to be invariant under permutations of spins between the groups. The state (3.32 is of course invariant under permutations of all $N$ spins.

${ }^{8}$ This follows from a straightforward generalization of the bipartite case, cf. (12) in 1],

$$
\left|N_{+}\right\rangle=\sum_{A_{+}+B_{+}+C_{+}+D_{+}=N_{+}} \sqrt{\left(\begin{array}{c}
A \\
A_{+}
\end{array}\right)\left(\begin{array}{c}
B \\
B_{+}
\end{array}\right)\left(\begin{array}{c}
C \\
C_{+}
\end{array}\right)\left(\begin{array}{c}
D \\
D_{+}
\end{array}\right) /\left(\begin{array}{c}
N \\
N+
\end{array}\right)}\left|A_{+}, B_{+}, C_{+}, D_{+}\right\rangle
$$

where the summation $\sum_{A_{+}+B_{+}+C_{+}+D_{+}=N_{+}}$is short hand for $\sum_{A_{+}}^{A} \sum_{B_{+}}^{B} \sum_{C_{+}}^{C} \sum_{D_{+}}^{D} \delta\left(A_{+}+B_{+}+C_{+}+D_{+}=N_{+}\right)$. 
given by

$$
\begin{aligned}
& X^{(4)}=g_{2} M+\frac{1}{2} \frac{1}{\left(1-n_{t}\right) n_{t}}[\operatorname{diag}(\alpha, \beta, \gamma, \delta)-M], \\
& Y^{(4)}=-\theta_{2} M,
\end{aligned}
$$

respectively, where the four by four matrix $M=(\alpha, \beta, \gamma, \delta) \otimes(\alpha, \beta, \gamma, \delta)$ denotes the dyadic square of the relative subsystem sizes, and $H_{2}^{\prime \prime}(x)=-[(1-x) x]^{-1}$ is the second derivative of the binary Shannon entropy.

To compute the entanglement entropies on the right hand side of (3.31), one needs the symplectic eigenvalues of the covariance matrix of the reduced Wigner function, cf. Eq. (3.26). Let $W_{A B C D}$ be the Wigner function of the quadripartite state $\Psi^{A B C D}$. The symplectic spectrum of the covariance $\Sigma$ of $W_{A B C D}$ is equal to $\{1 / 2\}$, because $W_{A B C D}$ comes from a pure state. The covariance matrices of the marginals of $W_{A B C D}$ are denoted by $\Sigma^{A}, \Sigma^{B}, \cdots \Sigma^{A B}, \cdots \Sigma^{A B C}$, etc. It turns out that exactly one symplectic eigenvalue of each marginal covariance is different from one half. This special symplectic eigenvalue is denoted by $\lambda_{A}, \lambda_{B}, \cdots \lambda_{A B}, \cdots \lambda_{A B C}$, etc., respectively. We refer to symplectic eigenvalues equal to one half as trivial symplectic eigenvalues, because they do not contribute to the entanglement entropy. Aided by a computer algebra system (Mathematica), we find

$$
\begin{aligned}
\lambda_{A} & =\frac{1}{2} \sqrt{1-2 C_{\alpha}+C_{\alpha} \frac{\operatorname{var} n_{+}}{\operatorname{var}_{B} n_{t}}+4 C_{\alpha} \operatorname{var}_{B}\left(n_{t}\right) \operatorname{var}(p)}, \quad C_{\alpha}=\alpha(1-\alpha), \\
\lambda_{A B} & =\frac{1}{2} \sqrt{1-2 C_{\alpha \beta}+C_{\alpha \beta} \frac{\operatorname{var} n_{+}}{\operatorname{var}_{B} n_{t}}+4 C_{\alpha \beta} \operatorname{var}_{B}\left(n_{t}\right) \operatorname{var}(p)}, \quad C_{\alpha \beta}=(\alpha+\beta)[1-(\alpha+\beta)],
\end{aligned}
$$

$\lambda_{A B C}=\lambda_{D}$, and analogously for the other combinations. Here, $\operatorname{var} n_{+}=\frac{1}{2 g_{2}}, \operatorname{var} p=\frac{g_{2}^{2}+\theta_{2}^{2}}{2 g_{2}}$ and $\operatorname{var}_{B} n_{t}=n_{t}\left(1-n_{t}\right)$ is the variance of a Bernoulli random variable with success probability $0<n_{t}<1$.

We elaborate on the observation that only one symplectic eigenvalue is non-trivial. For the sake of concreteness, we focus on the four by four matrix $\Sigma^{A B}$. Since only one of its two symplectic eigenvalues is nontrivial, it must be identical to the symplectic eigenvalue of the reduced covariance w.r.t. the bipartition into $A^{\prime}=A B$ and $B^{\prime}=C D$. Otherwise, this would contradict the result on the bipartite entanglement entropy $S\left(A^{\prime}\right)$. Indeed, the symplectic eigenvalues (3.35) are consistent with the symplectic eigenvalues in Eq. (24) of [1]. Hypothetically, $\Sigma^{A B}$ could also have two non-trivial symplectic eigenvalues, say $\lambda_{1}$ and $\lambda_{2}$, such that $S(A B)$ would be the sum of the two contributions $S\left(\lambda_{1}\right)$ and $S\left(\lambda_{2}\right)$, adding up to the bipartite entanglement $S\left(A^{\prime}\right)=S\left(\lambda_{1}\right)+S\left(\lambda_{2}\right)$, according to (3.26). If this was the case, then the way how $S\left(A^{\prime}\right)$ is distributed onto the summands $S\left(\lambda_{1}\right)$ and $S\left(\lambda_{2}\right)$ could be a characterization of entanglement beyond bipartite entanglement. However, the fact that only a single non-trivial symplectic eigenvalue in 3.35 carries all contribution to $S(A B)$, rules out this possibility.

The upshot is that any quadripartite entanglement measure that depends solely on the symplectic eigenvalues of the reduced covariance does not yield more information than already contained in bipartite entanglement measures for permutation invariant states. In this sense, the quadripartition is not more general than a bipartition for permutation invariant states.

We comment on the fact that the variance var $n_{+}$only occurs relative to $\operatorname{var}_{B} n_{t}$ in Eq. (3.35). In other words, the magnetization variance never occurs in absolute units, but only in units of the variance of a Bernoulli random variable with success probability $n_{t}$. This rather technical 


\section{Entanglement and magnetization variance in the transverse field Ising model}

detail is important and has a natural geometric interpretation on the Bloch sphere. Imagine the Bloch sphere embedded in three dimensional Euclidean space such that the south pole is at the origin and the north pole at $(0,0,1)$. Consider two (quasi) probability distributions on the Bloch sphere that differ only by a translation on the sphere, and are otherwise identical. For the sake of concreteness, assume, one is localized close to the north pole, the other is centered around a point close to the equator. The variance along the $z$-axis of the former is much smaller than the variance in $z$-direction of the latter. Not surprisingly, the variance in $z$ direction is not invariant under rotations of the Bloch sphere. However, as the entanglement is independent of the choice of the spin quantization axis, i.e. invariant under rotations of the Bloch sphere, the entanglement entropy cannot depend on the magnetization variance in $z$ direction directly. It turns out that the ratio between var $n_{+}$and $\operatorname{var}_{B} \mathbb{E} n_{+}=\mathbb{E} n_{+}\left(1-\mathbb{E} n_{+}\right)$is invariant under rotations of the sphere. The variance of the Bernoulli random variable vanishes at the poles and attains its maximum at the equator. Locally, this counteracts the transformation of the distances between two points on a meridian under projection onto the $z$-axis.

\subsection{Inhomogeneous initial states $*$}

The publication 1] exploits the fact that the Dicke subspace $\mathcal{D}_{N}$ of $\mathcal{H}_{N}$ is an invariant subspace for the unitary dynamics governed by the Schrödinger Eq. with a permutation invariant Hamiltonian. This symmetry allows to study the dynamics of permutation invariant states (in particular the dynamics of sudden quantum quenches) by analytic semiclassical methods, cf. chapter 2, In this section, we generalize the discussion to states that need not be fully permutation invariant, but may contain inhomogeneous structures in a specific sense, which we now explain.

Partition of spins. We define this larger class of inhomogeneous states as an enlargement of the Dicke subspace. Consider a partition of the set of $N$ spins into $k$ disjoint subsets $X_{1}, \cdots X_{k}$, each containing $N_{1}, \cdots N_{k}$ spins, respectively. Furthermore, we introduce the relative subsystems size $\alpha_{i}=N_{i} / N$ of subset $X_{i}$. Denoting the Dicke subspace of the subset $X_{i}$ by $\mathcal{D}_{X_{i}}$, we refer to the $k$ fold tensor product $\mathcal{D}_{\left\{X_{i}\right\}} \equiv \mathcal{D}_{\mathbf{X}}:=\bigotimes_{i=1}^{k} \mathcal{D}_{X_{i}}$ as the (k-partite) Dicke space relative to the partition $\mathbf{X}:=\left\{X_{i}\right\}_{i=1}^{k}$.

The nested relation between the full Hilbert space $\mathcal{H}_{N}$ (containing all, not necessarily permutation invariant, pure states), the Dicke subspace $\mathcal{D}_{\left\{X_{i}\right\}}$ relative to the partition $\left\{X_{i}\right\}$ (containing all pure states being invariant under permutation within each subset $X_{i}$ ), and the Dicke space $\mathcal{D}_{N}$ (containing all fully permutation invariant pure states), is

$$
\mathcal{D}_{N} \subset \mathcal{D}_{\left\{X_{i}\right\}} \subset \mathcal{H}_{N}
$$

Examples of states in $\mathcal{D}_{\left\{X_{i}\right\}}$ are products of permutation invariant state $\left|\psi_{i}\right\rangle$ on the subset $X_{i}$ of spins,

$$
\left|\psi_{1}\right\rangle \otimes \cdots \otimes\left|\psi_{k}\right\rangle
$$

and superpositions thereof. The Dicke basis of each factor $\mathcal{D}_{X_{i}}$ in $\mathcal{D}_{\left\{X_{i}\right\}}$ induces an orthonormal basis $\left\{\left|N_{1}^{(+)}, \cdots N_{k}^{(+)}\right\rangle\right\}$for the Dicke space relative to the partition $\mathbf{X}$. We refer to this basis as the Dicke basis of $\mathcal{D}_{\left\{X_{i}\right\}}$. Any state in $\mathcal{D}_{\left\{X_{i}\right\}}$ can be expanded in the Dicke basis as

$$
|\psi\rangle=\sum_{N_{1}^{(+)}, \cdots N_{k}^{(+)}} \psi\left(n_{1}, \cdots n_{k}\right)\left|N_{1}^{(+)}, \cdots N_{k}^{(+)}\right\rangle=\sum_{\mathbf{N}^{(+)}} \psi(\mathbf{n})\left|\mathbf{N}^{(+)}\right\rangle,
$$

$\mathbf{n}=\left(n_{1}, \cdots n_{k}\right)$ being the vector of up-spin fractions $n_{i}=N_{i}^{(+)} / N_{i}$ in subsystem $X_{i}$, and the 
summation is over all $k$-tuples in $\left\{\left(N_{1}^{(+)}, \cdots N_{k}^{(+)}\right): 0 \leq N_{i}^{(+)} \leq N_{i}\right\}$.

The discussion so far is somewhat parallel to the discussion of quadripartite entanglement in Sec. 3.6. The main difference is that the state (3.32), even though being expanded in the larger space $\mathcal{D}_{\mathbf{X}}$, is still fully invariant under all spin permutations and therefore in $\mathcal{D}_{N}$, while the general state (3.36) is only required to be invariant under permutations of spins within each subset $X_{i}$. The higher symmetry of (3.32) compared to (3.36) is manifest in the fact that the expansion coefficients in $(3.32)$ have a rigid form with less degrees of freedom.

Representation of permutation invariant operators on $\mathcal{D}_{\left\{X_{i}\right\}}$. We seek the matrix elements of permutation invariant spin operators on $\mathcal{H}_{N}$ in the Dicke basis of $\mathcal{D}_{\left\{X_{i}\right\}}$. Ultimately, we are interested in the matrix elements of the spin Hamiltonian (3.1). The collective spin operator: 9 $S_{x, y, z}=\sum_{i=1}^{N} s_{i}^{x, y, z} / N$ of $N$ spins decomposes into the convex linear combination $\sum_{j=1}^{k} \alpha_{j} S_{x, y, z}^{(j)}$ of collective spin operators $S_{x, y, z}^{(j)}=\sum_{i \in X_{j}} s_{i}^{x, y, z} / N_{j}$ of subsystem $X_{j}$. Employing the properties of the elementary spin one-half operators $s_{i}^{x, y, z}$, one verifies

$$
\begin{aligned}
S_{x}^{(j)}\left|\mathbf{N}^{(+)}\right\rangle & =\frac{1}{2} \sqrt{n_{j}\left(1-n_{j}+\frac{1}{N_{j}}\right)}\left|\mathbf{N}^{(+)}-\mathbf{e}_{j}\right\rangle+\frac{1}{2} \sqrt{\left(1-n_{j}\right)\left(n_{j}+\frac{1}{N_{j}}\right)}\left|\mathbf{N}^{(+)}+\mathbf{e}_{j}\right\rangle, \\
S_{y}^{(j)}\left|\mathbf{N}^{(+)}\right\rangle & =\frac{i}{2} \sqrt{n_{j}\left(1-n_{j}+\frac{1}{N_{j}}\right)}\left|\mathbf{N}^{(+)}-\mathbf{e}_{j}\right\rangle-\frac{i}{2} \sqrt{\left(1-n_{j}\right)\left(n_{j}+\frac{1}{N_{j}}\right)}\left|\mathbf{N}^{(+)}+\mathbf{e}_{j}\right\rangle, \\
S_{z}^{(j)}\left|\mathbf{N}^{(+)}\right\rangle & =\left(n_{j}-\frac{1}{2}\right)\left|\mathbf{N}^{(+)}\right\rangle,
\end{aligned}
$$

where $\left|\mathbf{N}^{(+)} \pm \mathbf{e}_{j}\right\rangle$ is short hand for $\left|N_{1}^{(+)}, \cdots N_{j-1}^{(+)}, N_{j}^{(+)} \pm 1, N_{j+1}^{(+)} \cdots N_{k}^{(+)}\right\rangle$. In particular, this shows that the collective operators $S_{x, y, z}^{(j)}$ leave $\mathcal{D}_{\mathbf{X}}$ invariant.

Effective Hamiltonian for inhomogeneous initial states. Consequently, the Hamiltonian (3.1), and thus the dynamics induced by the Schrödinger equation, is invariant on $\mathcal{D}_{\mathbf{X}}$. The matrix elements of $H$ follow readily from (3.37). Analogously to appendix A of [1], one derives ${ }^{10}$ the effective Schrödinger Eq. $i \hbar_{\text {eff }} \partial_{t} \psi_{t}(\mathbf{n})=H_{\text {eff }}(\mathbf{n}, \mathbf{p}) \psi_{t}(\mathbf{n})$, where, to leading order in $1 / N$,

$$
H_{\mathrm{eff}}(\mathbf{n}, \mathbf{p})=-\frac{J}{2}\left(\boldsymbol{\alpha} \cdot \mathbf{n}-\frac{1}{2}\right)^{2}-\Gamma \sum_{j=1}^{k} \alpha_{j} \sqrt{\left(1-n_{j}\right) n_{j}} \cos \left(\alpha_{j} p_{j}\right)
$$

cf. appendix A of $[1$

\footnotetext{
${ }^{9}$ The scaling factor of $1 / N$ in $S_{x, y, z}$ makes the spin operator intensive. This is important for the derivation of the effective description as $N \rightarrow \infty$, cf. Sec. 2.2 .

${ }^{10}$ First note, that the Schrödinger Eq. imposes

$$
\begin{aligned}
\frac{i}{N} \partial_{t} \psi_{t}(\mathbf{n})= & -\frac{J}{2}\left(\boldsymbol{\alpha} \cdot \mathbf{n}-\frac{1}{2}\right)^{2} \psi(\mathbf{n}) \\
& -\Gamma \sum_{j}^{k} \frac{\alpha_{j}}{2}\left[\sqrt{\left(1-n_{j}\right)\left(n_{j}+1 / N_{j}\right)} \psi\left(\mathbf{n}+\mathbf{e}_{j} / N_{j}\right)+\sqrt{n_{j}\left(1-n_{j}+1 / N_{j}\right)} \psi\left(\mathbf{n}-\mathbf{e}_{j} / N_{j}\right)\right]
\end{aligned}
$$
}

( $\mathbf{e}_{j}$ being the $j$-th $k$-dimensional canonical unit vector) on the coefficients of the Dicke basis expansion. By Taylor expansion, $\psi\left(\mathbf{n} \pm \mathbf{e}_{j} / N_{j}\right)=\left[\exp \left( \pm i \alpha_{j} p_{j}\right) \psi\right](\mathbf{n})$, where $p_{j}=-i \partial_{n_{j}} / N$ is the momentum operator associated to the $j$-th coordinate direction $n_{j}$. Thus,

$$
\frac{i}{N} \partial_{t} \psi_{t}(\mathbf{n})=-\frac{J}{2}\left(\boldsymbol{\alpha} \cdot \mathbf{n}-\frac{1}{2}\right)^{2} \psi(\mathbf{n})-\Gamma \sum_{j}^{k} \alpha_{j}\left[\sqrt{\left(1-n_{j}\right) n_{j}} \cos \left(\alpha_{j} p_{j}\right)+\mathcal{O}\left(1 / N_{j}\right)\right] \psi(\mathbf{n}) .
$$




\section{Entanglement and magnetization variance in the transverse field Ising model}

$\hbar_{\mathrm{eff}}=1 / N, \mathbf{n}=\left(n_{1}, \cdots n_{k}\right)$, and $\mathbf{p}=\left(p_{1}, \cdots p_{k}\right)$ denotes the vector of conjugate momenta $p_{j}=$ $-i \hbar_{\mathrm{eff}} \partial_{n_{j}}$ of $n_{j}$. The Hamiltonian (3.38) is coupled among its coordinates $\mathbf{n}$, but not among the momenta $\mathbf{p}$. The coupling comes from the pairwise spin coupling terms $s_{i}^{z} s_{j}^{z}$ in (3.1). As a consistency check, Eq. (3.38) reduces to (3.2) for $k=1$.

The Hamiltonian (3.38) inherits critical points from its homogeneous counterpart at $k=1$. More precisely, $H_{\text {eff }}$ has critical points at the uniform coordinates $\mathbf{z}^{*}=\left(n^{*}, \cdots n^{*}, p^{*}, \cdots p^{*}\right)$, where $\left(n^{*}, p^{*}\right)$ is a critical point of $H_{\mathrm{eff}}(n, p)$ for $k=1$. This is because (3.38) becomes independent of $\boldsymbol{\alpha}$ and $k$ for uniform $\mathbf{n}=(n, \cdots n)$ and $\mathbf{p}=(p, \cdots p)$. Hence, just as in the one-dimensional situation of $k=1$, the stable critical point at $n_{\mathrm{PM}}^{*}=1 / 2$ for $\Gamma>\Gamma_{c}=1 / 2$ becomes degenerate at $\Gamma=1 / 2$ and bifurcates into two stable critical points $n_{\mathrm{FM}}^{*}=\frac{1}{2}\left(1 \pm \sqrt{1-4 \Gamma^{2}}\right)$ for $\Gamma<\Gamma_{c}$. The harmonic approximation of $H_{\text {eff }}$ around these stable critical points can be viewed as $k$ coupled harmonic oscillators. The normal frequencies of this system of coupled oscillators are the symplectic eigenvalues of the Hessian

$$
\left.\nabla^{2} H_{\mathrm{eff}}\right|_{\mathbf{z}^{*}}=\left(\begin{array}{cc}
H_{\mathrm{eff}}^{n n} & H_{\mathrm{eff}}^{n p} \\
\left(H_{\mathrm{eff}}^{n p}\right)^{T} & H_{\mathrm{eff}}^{p p}
\end{array}\right)
$$

where the $k$ by $k$ blocks are

$$
\begin{aligned}
& \left(H_{\mathrm{eff}}^{n n}\right)_{i j}=-J \alpha_{i} \alpha_{j}+\frac{\Gamma}{4}\left[\left(1-n^{*}\right) n^{*}\right]^{-3 / 2} \alpha_{i} \delta_{i j}, \\
& \left(H_{\mathrm{eff}}^{p p}\right)_{i j}=\Gamma\left[\left(1-n^{*}\right) n^{*}\right]^{1 / 2} \alpha_{i} \delta_{i j}, \text { and } \\
& \left(H_{\mathrm{eff}}^{n p}\right)_{i j}=0 .
\end{aligned}
$$

For $k>1$ the $k$ different symplectic eigenvalues of $\nabla^{2} H_{\text {eff }}$ are rather complicated functions of $\boldsymbol{\alpha}$. Generically, i.e. for typical $\boldsymbol{\alpha} \in[0,1]^{k}$ with $\|\boldsymbol{\alpha}\|_{1}=1$, the symplectic eigenvalues are linearly independent over the rationals, i.e. the system of coupled oscillators are off-resonance. Interestingly, thus, the vector of relative subsystems sizes $\boldsymbol{\alpha}$ provides a mean to tune the frequencies of the coupled oscillators.

Inhomogeneous effective Hamiltonian. Even more general, when considering permutation invariant states relative to a partition $\mathbf{X}$, the Hamiltonian driving the dynamics does not need to be fully permutation invariant. It suffices that the Hamiltonian is permutation invariant with respect to the given partition. Also in this case, the dynamics will be confined to the Dicke subspace $\mathcal{D}_{\mathbf{X}}$.

For example, one could allow for an inhomogeneous external transverse magnetic field $\Gamma$ in (3.1). Let us assume that the magnetic field acts uniformly with value $\Gamma=\Gamma_{j}$ on all spins of subset $X_{j}$, but may have different values for different subsets. Likewise, one can allow for different longitudinal couplings with intra coupling $J_{j j}$ between spins of the same subset $X_{j}$, and inter coupling $J_{i j}$ between the spins of two different subgroups $X_{i}$ and $X_{j}$. With these modifications the effective Hamiltonian describing the dynamics on $\mathcal{D}_{\mathbf{X}}$ reads

$$
H_{\mathrm{eff}}(\mathbf{n}, \mathbf{p})=-\frac{1}{2} \sum_{i, j=1}^{k} \alpha_{i} \alpha_{j} J_{i j}\left(n_{i}-1 / 2\right)\left(n_{j}-1 / 2\right)-\sum_{j=1}^{k} \alpha_{j} \Gamma_{j} \sqrt{\left(1-n_{j}\right) n_{j}} \cos \left(\alpha_{j} p_{j}\right) .
$$

Expectation and Variance. As $N \rightarrow \infty$ Eqs. (3.38) and (3.39) provide an effective semiclassical description of the quantum spin model (3.1) for invariant states in $\mathcal{D}_{\mathbf{X}}$. In contrast to the homogeneous situation of $k=1$ considered in [1], the phase space of the classical limit is $2 k$-dimensional and its coordinates are $\mathbf{z}=(\mathbf{n}, \mathbf{p})$. In what follows, we discuss the nearby orbit formalism for the order parameter and its variance in this higher dimensional phase space analogous to Sec. of [1]. 
The effective description is particularly useful for localized WKB-like states. More precisely, let us assume that the initial state (3.36) at time $t=0$ is of large deviation form $\psi_{0}(\mathbf{n}) \asymp \exp \left[-N f_{0}(\mathbf{n})\right]$ with rate function $f_{0}(\mathbf{n})$, and that $\Re f_{0}$ is unimodal with a unique global minimum at $\mathbf{n}=\mathbf{n}_{\mathrm{cl}}$. Then, the expectation of the collective spin operators $\widehat{\mathbf{S}}_{z}=\left(S_{z}^{(1)}, \cdots S_{z}^{(k)}\right)$ is to leading order given by

$$
\left\langle\mathbf{S}_{z}\right\rangle=\mathbf{s}_{\mathrm{cl}}+\mathcal{O}\left(N^{-1}\right),
$$

where $\mathbf{s}_{\mathrm{cl}}$ is obtained from $\mathbf{n}_{\mathrm{cl}}$ by shifting all $k$ components by $-1 / 2$. Equivalently, the operator $\widehat{\mathbf{N}}=\left(S_{z}^{(1)}+1 / 2, \cdots S_{z}^{(k)}+1 / 2\right)$, which counts the fraction of up spins in each subsystem, has expectation

$$
\langle\mathbf{N}\rangle=\mathbf{n}_{\mathrm{cl}}+\mathcal{O}\left(N^{-1}\right)
$$

Similarly, the expectation of the conjugate momentum operator $\mathbf{P}=-i \hbar_{\mathrm{eff}} \nabla_{\mathbf{n}_{+}}$is

$$
\langle\mathbf{P}\rangle=\mathbf{p}_{\mathrm{cl}}+\mathcal{O}\left(N^{-1}\right),
$$

where $\mathbf{p}_{\mathrm{cl}}=i \nabla f_{0}\left(\mathbf{n}_{\mathrm{cl}}\right)=-\Im \nabla f_{0}\left(\mathbf{n}_{\mathrm{cl}}\right)$ is real, because $\Re \nabla f_{0}\left(\mathbf{n}_{\mathrm{cl}}\right)=0$.

Higher order order corrections to the expectation value can be computed by the saddle point approximation, and has a diagrammatic interpretation, cf. Sec. 3.2. Another measure for the corrections to the expectation value is given by the (co)variance matrix

$$
\operatorname{var}(\mathbf{N}, \mathbf{N})_{i j}:=\left\langle\mathbf{N}_{i} \mathbf{N}_{j}\right\rangle-\left\langle\mathbf{N}_{i}\right\rangle\left\langle\mathbf{N}_{j}\right\rangle
$$

The $i$-th diagonal entry of $\operatorname{var}(\mathbf{N}, \mathbf{N})$ is the variance $\operatorname{var}\left(\mathbf{N}_{i}\right)=\left\langle\left(\mathbf{N}_{i}-\left\langle\mathbf{N}_{i}\right\rangle\right)^{2}\right\rangle$ of $\mathbf{N}_{i}$. To obtain the leading contribution of $\operatorname{var}\left(\mathbf{N}_{i}, \mathbf{N}_{j}\right)$ via a saddle point approximation, the rate function $f_{0}$ is Taylor expanded to second order around the minimum $\mathbf{n}_{\mathrm{cl}}$, i.e. $f(\mathbf{z}) \approx f\left(\mathbf{z}_{\mathrm{cl}}\right)+\left.\frac{1}{2}\left(\mathbf{z}-\mathbf{z}_{\mathrm{cl}}\right) \nabla^{2} f_{0}\right|_{\mathbf{z}_{\mathrm{cl}}}\left(\mathbf{z}-\mathbf{z}_{\mathrm{cl}}\right)$, and the resulting Gaussian integral is solved. Let the real and imaginary part of the $k$ by $k$ Hessian matrix $\left.\nabla^{2} f_{0}\right|_{\mathbf{z}_{\mathrm{cl}}}$ be denoted by $X$ and $Y$, then

$$
\operatorname{var}(\mathbf{N}, \mathbf{N})=\frac{1}{2 N} X^{-1}+\mathcal{O}\left(N^{-2}\right)
$$

Similarly, one can compute the variance of the momentum operator $\mathbf{P}$,

$$
\operatorname{var}(\mathbf{P}, \mathbf{P})=\frac{1}{2 N}\left(X+Y X^{-1} Y\right)+\mathcal{O}\left(N^{-2}\right)
$$

Equivalently, the leading order of $\operatorname{var}(\mathbf{N}, \mathbf{N})$ and $\operatorname{var}(\mathbf{P}, \mathbf{P})$ are the variance matrices of the position and momentum marginals of the Wigner transform $W(\mathbf{z})$ of the Gaussian state $\psi_{0}^{\text {Gauss }}(\mathbf{n}) \asymp$ $\exp \left[-\frac{N}{2} \mathbf{n}(X+i Y) \mathbf{n}\right]$. The Wigner transform of a Gaussian state is a Gaussian distribution on phase space with $2 k$ by $2 k$ covariance

$$
\Sigma_{0}=\frac{1}{2 N}\left(\begin{array}{cc}
X^{-1} & -X^{-1} Y \\
-Y X^{-1} & X+Y X^{-1} Y
\end{array}\right)
$$

cf. Eq. (4) in 1

cf. Eq. (5a)

in $[1$

cf. Eq. (5b) in 1

cf. appendix $\mathrm{F}$ of 1

The covariance matrices (3.41a) and $(3.41 \mathrm{~b})$ are the variance matrices of the position and momentum marginals of $W(\mathbf{z})$. The off-diagonal matrices in the block matrix (3.42) are identified as the leading contribution of $\operatorname{var}(\mathbf{N}, \mathbf{P})_{i j}:=\frac{1}{2}\left\langle\mathbf{N}_{i} \mathbf{P}_{j}+\mathbf{P}_{j} \mathbf{N}_{i}\right\rangle-\left\langle\mathbf{N}_{i}\right\rangle\left\langle\mathbf{P}_{j}\right\rangle$,

$$
\operatorname{var}(\mathbf{N}, \mathbf{P})=-\frac{1}{2 N} X^{-1} Y+\mathcal{O}\left(N^{-2}\right)
$$




\section{Entanglement and magnetization variance in the transverse field Ising model}

Dynamics of expectation and variance. Now, we elaborate on the time evolution of the expectation and the variance. We begin with the expectation value. The Schrödinger evolution of states in $\mathcal{D}_{\mathbf{X}}$ according to $H$ in $(3.1)$ is effectively, up to corrections suppressed by $1 / N$, approximated by the effective Schrödinger Eq. with effective Hamiltonian $H_{\text {eff }}$ in (3.38) and effective Planck constant $\hbar_{\mathrm{eff}}=1 / N$. Hence, to leading order in $\hbar_{\mathrm{eff}}$, and for early times, the leading contribution of the expectation $\langle\mathbf{N}(t)\rangle=\mathbf{n}_{\mathrm{cl}}(t)+\mathcal{O}\left(N^{-1}\right)$ evolves according to Hamilton's classical equations of motion

$$
\frac{d \mathbf{z}_{\mathrm{cl}}}{d t}=J \nabla_{\mathbf{z}} H_{\mathrm{eff}}\left(\mathbf{z}_{\mathrm{cl}}\right)
$$

w.r.t. the effective Hamiltonian, where $\mathbf{z}_{\mathrm{cl}}=\left(\mathbf{n}_{\mathrm{cl}}, \mathbf{p}_{\mathrm{cl}}\right)$ and $J$ is the standard $2 k$ by $2 k$ symplectic form. The associated classical symplectic flow $T_{t}$ maps an initial condition $z_{0}$ to the time evolved phase space point $z(t)=T_{t}\left(z_{0}\right)$ at time $t$.

cf. Sec. 3.2

in $[1$

Next, we state the time evolution of the leading contribution to the variance. The leading contribution of the covariance matrix $\Sigma$, cf. (3.42), evolves according to the nearby orbit approximation [90, 91]. In a nutshell, the idea is to linearize the Hamiltonian flow around the reference orbit $\mathbf{z}_{\mathrm{cl}}(t)$, and thereby determine how small deviations from this reference change in time. The evolution of deviations from the reference orbit entail the dynamics of the variance to leading order. More explicitly, let $\mathbf{z}^{\prime}(t)$ denote a trajectory nearby the reference orbit, that is $\mathbf{z}^{\prime}(t)=\mathbf{z}_{\mathrm{cl}}(t)+\delta \mathbf{z}(t)$ with $\delta \mathbf{z}(t)$ sufficiently small for all times of interest. Linearization of the classical equation of motion $\dot{\mathbf{z}}^{\prime}(t)=J \nabla H\left(\mathbf{z}^{\prime}\right)$ around $\mathbf{z}_{\mathrm{cl}}$ imposes the non-autonomous ODE $\delta \dot{\mathbf{z}}=J\left(\left.\nabla^{2} H_{\mathrm{eff}}\right|_{\mathbf{z}_{\mathrm{cl}}(t)}\right) \delta \mathbf{z}+\mathcal{O}\left(\delta \mathbf{z}^{2}\right)$. Denoting the Hessian matrix of $H_{\text {eff }}$ at $\mathbf{z}_{\mathrm{cl}}(t)$ by $K(t)=\left.\nabla^{2} H_{\mathrm{eff}}\right|_{\mathbf{z}_{\mathrm{cl}}(t)}$, the ODE for $\delta \mathbf{z}$ is solved by $\delta \mathbf{z}(t)=S_{t} \delta \mathbf{z}(0)$, where $S_{t}=\mathcal{T} \exp \left[J \int_{0}^{t} K\left(t^{\prime}\right) d t^{\prime}\right]$ is the time ordered exponential function. Moreover, $S_{0}=\mathrm{id}$, and $S_{t}$ is the fundamental matrix of the ODE

$$
\dot{S}_{t}=J K(t) S_{t}
$$

Equivalently, $S_{t}$ measures how deviations $\delta \mathbf{z}(0)$ from $\mathbf{z}_{\mathrm{cl}}(0)$ at time zero propagate to deviations $\delta \mathbf{z}(t)$ from $\mathbf{z}_{\mathrm{cl}}(t)$ at time $t$. In other words, $S_{t}=\left.\nabla T_{t}\right|_{\mathbf{z}_{\mathrm{cl}}(0)}$ is the Jacobian matrix of the symplectic flow evaluated at the initial point $\mathbf{z}_{\mathrm{cl}}(0)$. Hence, $S_{t}$ is a $2 k$ by $2 k$ symplectic matrix. Its inverse $S_{t}^{-1}=\left.\nabla T_{-t}\right|_{\mathbf{z}_{\mathrm{cl}}(t)}$ follows from taking the derivative of $T_{-t} \circ T_{t}=$ id at $\mathbf{z}_{\mathrm{cl}}(0)$, by applying the chain rule.

Eqs. (3.41a) to (3.41c) suggest that the Wigner transform of a large deviation state can be approximated by a Gaussian density $W_{0}(\mathbf{z})=\mathcal{N}_{\mathbf{z}_{\mathrm{cl}}(0)}^{\Sigma_{0}}(\mathbf{z})$ with mean $\mathbf{z}_{\mathrm{cl}}(0)$ and variance matrix $\Sigma_{0}$, cf. (3.40) and (3.42). According to the truncated Wigner approximation (TWA) 88] the time evolved Wigner function is approximated by the solution of the classical Liouville equation to leading order in the large $N$ limit. Hence, $W_{t}(\mathbf{z}) \approx W_{0}\left(T_{-t} \mathbf{z}\right)$.

Now, we apply the nearby orbit approximation on top of this TWA result. Intuitively, as $W_{0}(\mathbf{z})$ is localized at $\mathbf{z}_{\mathrm{cl}}(0)$ within a neighborhood of diameter $\mathcal{O}(1 / \sqrt{N})$, the time evolved density $W_{t}$ will be localized at $\mathbf{z}_{\mathrm{cl}}(t)$, at least for early times. Let us therefore assume that $\mathbf{z}=\mathbf{z}_{\mathrm{cl}}(t)+\delta \mathbf{z}(t)$ 
is close to $\mathbf{z}_{\mathrm{cl}}(t)$, such that $\delta \mathbf{z}(t)=\mathcal{O}(1 / \sqrt{N})$ is small. By straightforward calculation,

$$
\begin{aligned}
W_{t}(\mathbf{z}) & \approx W_{0}\left(T_{-t} \mathbf{z}\right) \\
& =\mathcal{N}_{\mathbf{z}_{\mathrm{cl}}(0)}^{\Sigma}\left(T_{-t} \mathbf{z}\right)+\mathcal{O}\left(\hbar_{\mathrm{eff}}\right) \\
& =\mathcal{N}_{\mathbf{z}_{\mathrm{cl}}(0)}^{\Sigma}\left[T_{-t}\left(\mathbf{z}_{\mathrm{cl}}(t)\right)+\left.\nabla T_{-t}\right|_{\mathbf{z}_{\mathrm{cl}}} \delta \mathbf{z}(t)+\mathcal{O}\left(\hbar_{\mathrm{eff}}\right)\right]+\mathcal{O}\left(\hbar_{\mathrm{eff}}\right) \\
& =\mathcal{N}_{0}^{\Sigma}\left[S_{t}^{-1} \delta \mathbf{z}(t)\right]+\mathcal{O}\left(\hbar_{\mathrm{eff}}\right) \\
& =\mathcal{N}_{0}^{S_{t} \Sigma S_{t}^{T}}[\delta \mathbf{z}(t)]+\mathcal{O}\left(\hbar_{\mathrm{eff}}\right) \\
& =\mathcal{N}_{\mathbf{z}_{\mathrm{cl}}(t)}^{S_{t} \Sigma S_{t}^{T}}(\mathbf{z})+\mathcal{O}\left(\hbar_{\mathrm{eff}}\right)
\end{aligned}
$$

Hence, to leading order, the time evolved Wigner function remains a Gaussian centered at the classical trajectory $\mathbf{z}_{\mathrm{cl}}(t)$ of $(3.43)$ with variance

$$
\Sigma_{t}=S_{t} \Sigma S_{t}^{T}
$$

where $S_{t}$ solves (3.44).

Instead of solving (3.44) for $S_{t}$ and then obtaining $\Sigma_{t}$, one can also solve

$$
\begin{gathered}
\dot{X}_{m n}=-H_{, m}^{, j} X_{j n}-H_{, n}^{, j} X_{j m}+H^{, j l}\left(X_{j m} Y_{l n}+Y_{j m} X_{l n}\right) \quad \text { and } \\
\dot{Y}_{m n}=H_{, m n}-H_{, m}^{, j} Y_{j n}-H_{, n}^{, j} Y_{j m}-H^{, j l}\left(X_{j m} X_{l n}-Y_{j m} Y_{l n}\right)
\end{gathered}
$$

for the $k$ by $k$ matrices $X$ and $Y$ (the real and imaginary part of the Hessian matrix of $f$ at $\mathbf{x}_{\mathrm{cl}}(t)$ ), and obtain $\Sigma_{t}$ from (3.42). The summation over repeated indices in (3.46) is implicit. Moreover, $H_{, n}^{, m}=\left.\partial_{x^{n}} \partial_{p_{m}} H_{\mathrm{eff}}(\mathbf{x}, \mathbf{p})\right|_{\mathbf{x}_{\mathrm{cl}}, \mathbf{p}_{c} l}$ and similarly for $H^{, m n}$ and $H_{, m n}$ denotes the derivative of the effective Hamiltonian w.r.t. positions and momenta, cf. appendix C. Eq. (3.46) is derived in appendix C by a multivariate rate function expansion, and is the higher dimensional analog of Eq. (3.13a) and appendix B in [1].

The equivalence of $(3.45)$ and $(3.46)$ was proven for the one-dimensional case (i.e. $k=1$ ) in appendix $\mathrm{C}$ of [1], and generalizes mutatis mutandis to $k>1$. In other words, the diagram

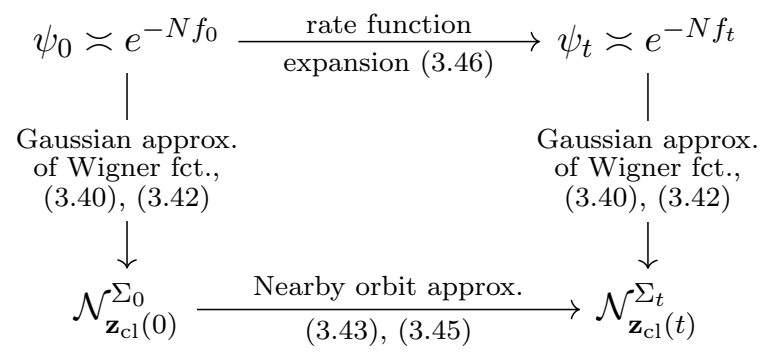

commutes.

The rate function expansion approach can also be used to compute higher order cumulants beyond the variance, analogously to Sec. 3.2 .

Discussion. We contemplate on the result (3.38) and close this section with some speculations.

Permutation invariant states of $N$ spins are described by a single good quantum number (the number of up spins), and the quantum evolution with permutation invariant Hamiltonian (3.1) leads to an effective one-dimensional semiclassical description in the large $N$ limit. As the effective Hamiltonian (3.2) is time independent, the classical limit is trivially integrable, and trivially ergodic on one-dimensional energy hypersurfaces.

In contrast, if the initial state is not fully permutation invariant, but only permutation invariant 


\section{Entanglement and magnetization variance in the transverse field Ising model}

within $k$ disjoint subsets of its elementary spins, the state requires $k$ independent good quantum numbers, and the quantum dynamics is described by an effective $k$-dimensional semiclassical model, cf. (3.38). Hamiltonian systems on higher dimensional phase spaces are richer than their onedimensional counterparts. In particular, classically chaotic behavior, in the sense of exponentially deviating orbits, can only occur for phase space dimensions above two, cf. Sec. 2.3 .

What are the properties of the classical Hamiltonian system (3.38)? Close to the stable critical point with homogeneous coordinates $\mathbf{z}^{*}=\left(n_{\mathrm{cl}} \cdots n_{\mathrm{cl}}\right)$ the Hamiltonian can be expanded to quadratic order. Hence, one may expect the behavior of coupled oscillators, i.e. classically integrable behavior on $k$-dimensional invariant tori, close to $\mathbf{z}^{*}$. How do the invariant tori get deformed in regions of phase space, where non-harmonic correction terms become important? Do the tori become unstable, leading to classically fully chaotic regions in phase space? As has been alluded to above, the frequencies of the oscillators depend on the relative subsystem size vector $\boldsymbol{\alpha}$. Thus the subsystem size $\boldsymbol{\alpha}$ may provide a tunable parameter to adjust the commensurability of the oscillator frequencies. Therefore, $\boldsymbol{\alpha}$ could potentially be used to control the integrability breaking according to the KAM theorem.

What are the implications for the spin model (3.1)? How do the results on the variance and entanglement in 11 get modified? For small inhomogeneities in the initial state and small quenches, one may expect that the effective dynamics remains close to the homogeneous subspace. In this case the Wigner function evolves within a classically integrable phase space region of (deformed) invariant tori. The magnetization variance and the entanglement is described by the higher dimensional analogs of [1, as discussed in this section and Sec. 3.4.

For strong initial inhomogeneities and for large quenches the effective dynamics may be very different from the homogeneous situation of [1]. Depending on the precise phase space structure of the effective Hamiltonian, inhomogeneities may also be enhanced and evolve in time. The underlying effective dynamics may also be classically chaotic. A more thorough investigation is necessary.

\subsection{Publication [1]}

The author's contribution. The idea to investigate the dynamics of the order parameter variance in the fully connected transverse field Ising model after a quantum quench with a large deviation ansatz is due to Stefan Kehrein. All analytical an numerical calculations have been done by the author of this thesis. He has also written the paper. The author has profited a lot from joint scientific discussions with Stefan Kehrein.

Copyright. The article has been submitted for publication under the creative commons license 'Attribution 4.0 International (CC BY 4.0)' to SciPost Physics. 


\title{
Out of equilibrium mean field dynamics in the transverse field Ising model
}

\author{
I. Homrighausen ${ }^{1}$, S. Kehrein ${ }^{1 *}$, \\ 1 Universität Göttingen, Institute for Theoretical Physics, \\ Friedrich-Hund-Platz 1, 37077 Göttingen, Germany \\ * stefan.kehrein@theorie.physik.uni-goettingen.de
}

October 24, 2019

\begin{abstract}
We investigate the quench dynamics of the transverse field Ising model on a finite fully connected lattice. Using a rate function approach we compute the leading order corrections to the mean field behavior analytically. Our focus is threefold: i) We analyze the validity of the mean field approximation and observe that deviations can occur quickly even for large systems. ii) We study the variance of the order parameter and identify four dynamically qualitative different regions. iii) We derive the entanglement Hamiltonian for a bipartition of the lattice, which turns out to be a time-dependent harmonic oscillator.
\end{abstract}

\section{Contents}

1 Introduction $\quad 2$

2 Mean field models 5

2.1 Transverse field Ising model 5

2.2 Dicke subspace and effective Hamiltonian 5

3 Semiclassics $\quad 6$

3.1 Rate function expansion $\quad 6$

$\begin{array}{lll}3.2 & \text { Phase space picture } & 7\end{array}$

4 Results for the variance $\quad 8$

4.1 Exponential growth regime (I) 11

4.2 Periodic regime (II) 11

\begin{tabular}{ll}
4.3 & Quadratic growth regime (III) \\
\hline
\end{tabular}

4.4 Periodically enhanced squeezing regime (IV) 12

4.5 Validity of the mean field approximation in non-equilibrium 14

5 Entanglement dynamics $\quad \mathbf{1 5}$

$\begin{array}{lll}5.1 & \text { Bipartition and reduced density matrix } & 16\end{array}$

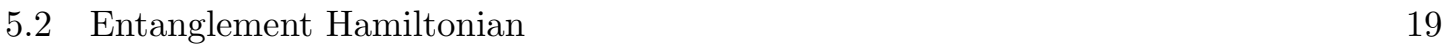

5.3 Rényi entanglement entropies 20 
5.4 Dynamics of entanglement 22

6 Conclusion 25

A Derivation of the effective Hamiltonian 28

B Rate function expansion $\quad 29$

$\begin{array}{ll}\text { C Nearby orbit vs. large deviation } & 31\end{array}$

D Nearby orbit approximation for periodic orbits 32

D.1 Interpretation of $\alpha \quad 34$

$\begin{array}{lll}\text { D.2 Example } & 35\end{array}$

$\begin{array}{ll}\text { E Nearby orbit approximation at fixed points } & 36\end{array}$

$\begin{array}{lll}\text { F Wigner function of Gaussian density } & 37\end{array}$

G Replica trick $\quad 38$

H Spin squeezing and entanglement $\quad 40$

References $\quad 42$

\section{Introduction}

One of the reasons why quantum mechanical many body systems are difficult to analyze is because the dimension of the Hilbert space grows exponentially with the number of particles. In contrast, the dimension of classical phase space scales only linear in the particle number. Another unique feature of quantum mechanics is entanglement, which has no immediate classical analog $[1,2]$. When entanglement of a composite system is measured by means of the von Neumann entanglement entropy, the logarithm of the Hilbert space dimension of the smaller subsystem is an upper bound on the entanglement. Turning this intuition around, one can view the exponential of the entanglement entropy as the effective dimension in which the entangled state lives. In this sense, the combination of both, large entanglement and exponential Hilbert space dimension, makes the quantum time evolution computationally challenging. Many numerical algorithms, such as the density matrix renormalization group $[3-5]$ with matrix product states [6], rely on the fact that the entanglement of the states of interest remains low such that the effective Hilbert space dimension is small and the complexity of the exponential dimension is effectively avoided. Generic quantum many body systems are not exactly solvable and one is restricted to numerical methods and finite computational resources. From this perspective, it is vital to understand how entanglement grows in time in non-equilibrium situations.

The entanglement dynamics after a global quantum quench has been investigated for numerous local Hamiltonians. Linear growth of the entanglement entropy has been observed 
for one dimensional gapped lattice systems [7], conformal field theories [8,9], non integrable spin chains [10], and harmonic oscillator chains [11]. This typically limits the study to low dimensional locally interacting systems, for which the area law [12-16] guarantees low entanglement entropy in the ground state, and to small system sizes at early times. The linear growth of entanglement as discussed in [7-9,17], is mediated by quasiparticles propagating in a Lieb-Robinson cone formed by a maximal group velocity. The quasiparticle picture has been confirmed analytically in integrable models $[8,9]$, as well as numerically, e.g. by looking at the mutual information between two spatially separated places [18], or the particle number fluctuation [19]. There are, however, exceptions to the connection between entanglement growth and the spread of quasiparticles. On the one hand, it is known [10] that some non integrable models show linear entanglement growth, while the energy transport, being mediated by quasiparticles, is only diffusive. On the other hand, sublinear entanglement growth was observed in geometric quenches, even though quasiparticles spread ballistically [20].

A notable exception to linear entanglement growth in short range systems are disordered models that exhibit many body localization and show logarithmic entanglement growth [2123]. The logarithmic growth can be argued to be a consequence of a dephasing mechanism facilitated by exponentially decaying interactions between localized quasiparticles [23].

In addition to short range models, systems with long range interaction have gained theoretical [24-33], as well as experimental interest due to their realization with ultra cold atoms $[34,35]$. Another, more theoretical, motivation to study long range models is to use them as an approximate equivalent for high dimensional short range systems [19]. It has been found numerically $[18,36,37]$ and semi-analytically $[27,28]$ that the entanglement entropy grows only logarithmically in time, that is much slower than their short range counterpart. A heuristic, non-quantitative argument in favor of the logarithmic growth [27], also see [19], relies on the fact that the maximal group velocity diverges for the $k=0$ mode, while the density of states vanishes as $k \rightarrow 0$. This leads to a breakdown of a pronounced light cone, and information is only propagated slowly by quasiparticles. However, this line of reasoning cannot be applied to the limiting case of uniform all to all coupling, because fully connected models lack the notion of spatial distance and a quasiparticle picture.

In the present paper, we look at the out of equilibrium dynamics in an infinite range, highly symmetric model, which becomes amenable to a mathematically controlled expansion in the thermodynamic limit. More precisely, we focus on a spin system defined on a fully connected lattice, being invariant under permutations of lattice sites. For the sake of concreteness, we will focus on the fully connected transverse field Ising model (also known as the Lipkin Meshkov Glick model [38]), however, the mathematical reasoning also applies to other meanfield models on fully connected lattices [39].

Mean field models, and mean field approximations of more complicated systems provide an accessible approach to study many body problems, both, in classical, and quantum statistical physics. The applications of mean field approximations in equilibrium situations are numerous, and it is rather well understood when mean field yields reliable results. In contrast, mean field approximations are less frequently used in non equilibrium conditions, and it is not generally known when and how well mean field works. From this point of view, the transverse field Ising model serves as a basic and non-trivial example to study the validity of approximations out of equilibrium. Two advantages of this specific model are that, first, it is accessible to controlled analytical calculations, and, second, because numerically exact solutions for large system sizes are feasible, it is possible to compare the approximations to exact results. One of the surprising findings is how short the time scale of validity of the mean field 
approximation in this system is. More specifically, we show that, away from critical points, mean field is only reliable for early times of the order of the square root of the system size. And, close to unstable critical points, the mean field approximation already breaks down on timescales logarithmic in system size.

When driving the fully connected Ising model out of equilibrium by means of a sudden quantum quench, the dynamics is constrained to the site permutation invariant subspace, which is referred to as the Dicke subspace. The dimension of the Dicke subspace scales linearly with the number of spins, which reminds of the scaling of classical phase spaces. Indeed, permutation invariance facilitates the use of semiclassical techniques. In this way, the quench dynamics in the transverse field Ising model on a fully connected lattice becomes amenable to a mathematically controlled expansion around the classical limit, and is a useful test case to benchmark the validity of mean field type approximations out of equilibrium.

Spin systems on fully connected lattice geometries can be viewed as a single collective spin, and are thus mathematically equivalent to the two mode Bose Hubbard model $[40,41]$ via the Jordan-Schwinger mapping $[42,43]$. The two mode Bose Hubbard model is experimentally realized as a Bose-Einstein condensate (BEC) using ultra cold atoms in optical traps [34,35]. In this context, entanglement between the two modes has been investigated theoretically [29-32] and experimentally [35,44]. A typical entanglement measure between the modes of a dimer is referred to as EPR-entanglement. Besides the entanglement between the two modes of a BEC dimer, one may also investigate the entanglement between different particles of the BEC, which corresponds to a different bipartition of the Hilbert space [30]. In this paper, we focus on the entanglement between particles.

Although being a relatively simple model, the entanglement dynamics in the mean field Ising model is non-trivial and exhibits qualitatively different behavior, such as linear growth, logarithmic growth, and bounded oscillations, depending on the initial pre-quench state and the final post-quench Hamiltonian. Remarkably, within the validity of the mean-field approximation we can analytically derive the complete entanglement Hamiltonian in leading order, which turns out to be a time-dependent harmonic oscillator. This provides a rare case where the complete entanglement Hamiltonian and therefore all Rényi entanglement entropies are analytically known for a non-trivial quantum many body system. The dynamical behavior can be understood by making use of an intimate connection between entanglement and spin squeezing [33, 40, 45-49].

Throughout the paper, we compare analytical predictions to numerical data obtained by exact diagonalization, and find excellent agreement at early times. The fact that the Dicke subspace dimension scales linearly with the number of spins, allows one to solve systems of $10^{4}$ spins numerically exact. However, even for large system sizes a dephasing mechanism leads to a deviation from the mean field approximation as time proceeds.

This article is structured as follows. The fully connected transverse field Ising model is defined in Sec. 2, and the mapping to an effective semiclassical model in the limit of large system size is explained. In Sec. 3, two semiclassical techniques, one based on a rate function expansion, the other based on deviations between classical trajectories, is reviewed. These techniques are used in the discussion of the quench-induced dynamics of the mean magnetization and its variance, see Sec. 4 , and the entanglement entropy with respect to a bipartition of spins, see Sec. 5. The dynamical phase diagram based on the behavior of the order parameter and the variance is discussed in Sec. 4 and entanglement is analyzed in Sec. 5. The article concludes with Sec. 6 . 


\section{Mean field models}

\subsection{Transverse field Ising model}

We investigate the transverse field Ising model on a fully connected graph of $N$ sites given by the Hamiltonian

$$
\mathcal{H}=-\frac{J}{2 N} \sum_{i, j} s_{i}^{z} s_{j}^{z}-\Gamma \sum_{i} s_{i}^{x}
$$

where $s_{i}^{x, y, z}=\sigma_{i}^{x, y, z} / 2$ denotes the spin $1 / 2$ representation of the spin at site $i$ in terms of the Pauli matrices, $\Gamma$ is the homogeneous transverse field, and $J>0$ denotes the ferromagnetic coupling. Note that the double sum is rescaled by a factor of $1 / N$ in order to make it of the same order of magnitude as the single sum. In this way, both terms, the ferromagnetic term and the transverse term, scale linear with the system size such that the Hamiltonian is extensive. The linear scaling becomes more apparent when introducing the (rescaled) total spin operators $S_{x, y, z}=\sum_{i} s_{i}^{x, y, z} / N$ in terms of which the Hamiltonian (1) reads

$$
\mathcal{H}=-N J S_{z}^{2} / 2-N \Gamma S_{x}
$$

The factor of $1 / N$ in the definition of $S_{x, y, z}$ is chosen such that its spectrum consists of $(N+1)$ equidistant points contained in the interval $[-1 / 2,1 / 2]$. One can thus view $S_{x, y, z}$ as a quantity of order one as $N \rightarrow \infty$. Note that $S_{x, y, z}$ obey the usual $S U(2)$ commutation relations decorated with an additional factor of $\hbar_{\text {eff }}:=1 / N$. In the sequel, we choose units of time and energy in which $\hbar=1$ and $J=1$.

\subsection{Dicke subspace and effective Hamiltonian}

The Hamiltonian (1) is defined on the Hilbert space $\mathcal{H}_{N}=\bigotimes^{N} \mathbb{C}^{2}$. The tensor products $\left|s_{1}, \ldots, s_{N}\right\rangle$ of the $s_{i}^{z}$ eigenstates $\left|s_{i}= \pm 1 / 2\right\rangle$ form an orthonormal basis of $\mathcal{H}_{N}$. An important subspace of $\mathcal{H}_{N}$ is the Dicke space $\mathcal{D}_{N}$ containing all states that are invariant under permutations of spins. A convenient orthonormal basis of $\mathcal{D}_{N}$ is given by the Dicke states $\left\{\left|N_{+}\right\rangle\right\}_{N_{+}=0, \ldots, N}$, being defined as the superposition of all spin permutations with exactly $N_{+}$ of $N$ spins being up,

$$
\left|N_{+}\right\rangle=\left(\begin{array}{c}
N \\
N_{+}
\end{array}\right)^{1 / 2} P\left(|\uparrow\rangle^{\otimes N_{+}} \otimes|\downarrow\rangle^{\otimes N-N_{+}}\right)
$$

where $P$ denotes the projection operator $P\left|s_{1}, \ldots s_{N}\right\rangle=\frac{1}{N !} \sum_{p \in \mathcal{S}_{N}}\left|s_{p(1)} \ldots s_{p(N)}\right\rangle$ and $S_{N}$ denotes the symmetric group on $N$ symbols. The Dicke state $\left|N_{+}\right\rangle$is the permutation invariant eigenstate of $S_{z}$ with eigenvalue $\left(n_{+}-1 / 2\right)$. Note that $\mathcal{D}_{N}$ is $(N+1)$ dimensional, i.e. its dimension scales linearly with the system size, as opposed to the exponential scaling of the $2^{N}$ dimensional total Hilbert space $\mathcal{H}_{N}$. The fact that the dimension of $\mathcal{D}_{N}$ scales only linear in $N$ allows to study the dynamics using exact diagonalization for large systems of the order of $N=10^{4}$.

In this paper we study the non-equilibrium dynamics after a sudden quantum quench $\Gamma_{i} \rightarrow \Gamma_{f}$ in the magnetic field. That is to say, the system is prepared in the ground state $\left|\Psi_{0}\right\rangle$ of the pre-quench Hamiltonian $\mathcal{H}\left(\Gamma_{i}\right)$ and is evolved with the post-quench Hamiltonian $\mathcal{H}\left(\Gamma_{f}\right)$ according to the Schrödinger equation. On a fully connected lattice, both, the Hamiltonian (1) as well as the ground state, are invariant under spin permutations. Hence, in a quench 
setup, the dynamics is confined to $\mathcal{D}_{N}$ and the wave function can be expanded in terms of the Dicke states as

$$
|\psi\rangle=\sum_{N_{+}=0}^{N} \psi\left(n_{+}\right)\left|N_{+}\right\rangle
$$

( $n_{+}$being $\left.N_{+} / N\right)$.

The time dependent Schrödinger equation $i \partial_{t}|\Psi\rangle=\mathcal{H}|\Psi\rangle$ imposes the dynamics

$$
i \hbar_{\mathrm{eff}} \partial_{t} \psi\left(n_{+}\right)=H\left(n_{+}, p\right) \psi\left(n_{+}\right)
$$

on the coefficients $\psi\left(n_{+}\right)=\left\langle N_{+} \mid \psi\right\rangle$ with the effective Hamiltonian

$$
H\left(n_{+}, p\right)=-\frac{1}{2}\left(n_{+}-1 / 2\right)^{2}-\Gamma \sqrt{n_{+}-n_{+}^{2}} \cos (p),
$$

where $p=-i \hbar_{\mathrm{eff}} \partial_{n_{+}}$and $\hbar_{\mathrm{eff}}=1 / N$. Details on the derivation of the effective Hamiltonian are given in Appendix A and [39], also see [50-52] for a derivation in the context of Bose-Einstein condensate dimers starting from a Gross-Pitaevski description. The effective description by Eq. (2) is an approximation because of two reasons. First, additional terms in $H\left(n_{+}, p\right)$ that are suppressed by $1 / N$ are neglected. Second, the discrete nature of $n_{+}$(taking values in $\{0,1 / N, \ldots 1\})$ is approximated by treating $n_{+}$as a continuous variable with values in the unit interval $[0,1]$. These approximations are believed to be valid as $N \rightarrow \infty$. Equation (2) has the form of an effective one dimensional single particle Schrödinger equation for a fictitious particle. The position of the fictitious particle is given by the fraction $n_{+}=N_{+} / N$ of up-spins, and the conjugate momentum $p=-i \hbar_{\mathrm{eff}} \partial_{n_{+}}$can be interpreted as the polar angle on the Bloch sphere. As the effective Planck constant $\hbar_{\text {eff }}$ is the inverse system size, we may exploit semiclassical techniques in the large system limit to investigate the non-equilibrium dynamics after a sudden quench.

\section{Semiclassics}

Two semiclassical methods are presented. First, in the subsequent section, a systematic rate function expansion akin to WKB theory is discussed. This method gives a systematic $1 / N$-expansion of the expectation value and the variance of observables and their dynamics. The main result will be Eq. (7), which is a simple ordinary differential equation describing the dynamics of the leading contribution to the variance. Second, thereafter in section 3.2 , a semiclassical phase space approach, known as nearby orbit approximation [53,54], is reviewed. This method is particularly suited to facilitate an intuitive way of thinking and complements the less intuitive rate function expansion. We will take great advantage of this phase space picture when we explain the periodically enhanced spin squeezing. Both methods, the rate function expansion and the nearby orbit approximation, give identical results for the leading order term of the variance. This equivalence is proved in Appendix C.

\subsection{Rate function expansion}

In the large $N$ limit the ground state of (2b) may be approximated by WKB-type states [55-57] of large deviation form

$$
\psi\left(n_{+}\right) \asymp e^{-N f\left(n_{+}\right)}
$$


with $N$-independent complex rate function $f\left(n_{+}\right)$[39]. Following the notation of $[58,59]$, we write $a \asymp b$ to denote that two quantities are equal to first order in their exponents, i.e. $\lim _{N \rightarrow \infty} \frac{1}{N} \log a / b=0$. The modulus of $\psi\left(n_{+}\right)$is localized around the minimum of $\Re f\left(n_{+}\right)$. We assume that $\Re f\left(n_{+}\right)$has a unique global minimum denoted by $n_{\mathrm{cl}}$. The expectation values $\left\langle n_{+}\right\rangle$and $\langle p\rangle$ in the state (3) follow from a leading order saddle point approximation to be

$$
\begin{aligned}
\left\langle n_{+}\right\rangle & =n_{\mathrm{cl}}+\mathcal{O}(1 / N), \\
\langle p\rangle & =p_{\mathrm{cl}}+\mathcal{O}(1 / N),
\end{aligned}
$$

where $p_{\mathrm{cl}}=i f^{\prime}\left(n_{\mathrm{cl}}\right)$. Moreover, the curvature of the rate function at $n_{\mathrm{cl}}$ determines the variance $\operatorname{var}\left(n_{+}\right)=\left\langle\left(n_{+}-\left\langle n_{+}\right\rangle\right)^{2}\right\rangle$ and $\operatorname{var}(p)=\left\langle(p-\langle p\rangle)^{2}\right\rangle$. If we denote the second derivative $f^{\prime \prime}\left(n_{\mathrm{cl}}\right)$ by $f_{2}$, we have

$$
\begin{aligned}
\operatorname{var}\left(n_{+}\right) & =\frac{1}{2 N}\left(\Re f_{2}\right)^{-1}+\mathcal{O}\left(1 / N^{2}\right), \\
\operatorname{var}(p) & =\frac{1}{2 N}\left[\Re\left(f_{2}^{-1}\right)\right]^{-1}+\mathcal{O}\left(1 / N^{2}\right) .
\end{aligned}
$$

Likewise, all higher moments may be computed systematically in this perturbative manner by the saddle point approximation.

Now, we investigate the time evolution of the expectation value and its variance to leading order in $1 / N$. In order to avoid ordering ambiguities, we assume that the Hamiltonian $H\left(n_{+}, p\right)$ in (2) is normal ordered in the sense that the momentum operator $p$ is commuted to the right. Then, the effective Schrödinger equation (2) imposes the partial differential equation

$$
\partial_{t} f\left(n_{+}, t\right)=i H\left(n_{+}, i \partial_{n+} f\left(n_{+}, t\right)\right)+\mathcal{O}(1 / N)
$$

on the rate function. Consequently, the quantities $n_{\mathrm{cl}}, p_{\mathrm{cl}}$, and $f_{2}$ become time dependent. As was shown in [39] $n_{\mathrm{cl}}(t)$ and $p_{\mathrm{cl}}(t)$ obey the classical Hamiltonian equations with Hamiltonian $H$. Elaborating on this result, we derive the differential equation

$$
i \frac{d f_{2}}{d t}=-\left(1, i f_{2}\right) H^{\prime \prime}\left(1, i f_{2}\right)
$$

for $f_{2}$, where $H^{\prime \prime}$ is the two by two Hessian matrix of $H\left(n_{+}, p\right)$ evaluated at $n_{+}=n_{\mathrm{cl}}, p=p_{\mathrm{cl}}$ in Appendix B. The time-dependence of $f_{2}$ yields the dynamics of $\operatorname{var}\left(n_{+}\right)$and $\operatorname{var}(p)$ according to Eq. (5). It is a non-trivial fact that the time evolution of the variances does not depend on higher moments, such as the skewness, but only on the expectation values. This is a special case of a more general result. Namely, that the dynamics of the leading order of the $n$th moment depend only on moments of order smaller than $n$ (more details in Appendix B).

\subsection{Phase space picture}

The preceding paragraph introduced a systematic large $N$ expansion of the rate function. The computation of the variance is reduced to the solution of the ordinary differential equation (7) of the rate function's curvature at the classical trajectory. In the present paragraph we introduce a complementary semiclassical technique, which is based on a phase space picture.

The idea of a phase space formulation of quantum mechanics has a long-standing history and goes back to Wigner and Moyal $[60,61]$. In a nutshell, phase space methods map the quantum mechanical wave function to a quasi-probability distribution on phase space whose 
dynamics is then inherited from the Schrödinger equation [62-64]. One of the most commonly used quasi-probability distribution is the Wigner function and its evolution is governed by Moyal's equation. Operator expectation values are then obtained by integrating the Weyl symbol of that operator against the Wigner function over the whole phase space.

The leading contribution as $\hbar_{\mathrm{eff}} \rightarrow 0$ of the Moyal equation is the classical Liouville equation. Corrections to the Liouville's equation are suppressed by at least $\hbar_{\text {eff }}^{2}[60]$. As we are only interested in the leading order dynamics as $1 / N \rightarrow 0$, we will approximate the Moyal equation by Liouville's equation. This is sometimes referred to as the truncated Wigner approximation and it is exact for quadratic Hamiltonians. As innocent as this approximation seems, it is known that the limit $\hbar_{\text {eff }} \rightarrow 0$ may have an essential singularity and the truncated Wigner approximation may be insufficient in this case [65]. This issue, however, is less relevant for us, as we consider only those quenches, for which the initial Wigner function can be approximated by a single Gaussian. The mean of this initial Gaussian is given by $\left(n_{\mathrm{cl}}(0), p_{\mathrm{cl}}(0)\right)$ (compare Eq. (4)), and the covariance matrix $C(0)$ is diagonal with eigenvalues $\left[\Re\left(2 N f_{2}(0)\right)\right]^{-1}$ and $\Re\left[\left(2 N f_{2}(0)\right)^{-1}\right]$ (compare Eq. (5)). As the initial Wigner function is strongly localized, on a scale of $1 / \sqrt{N}$ in phase space, the nearby orbit approximation $[53,54]$ predicts that the evolved Wigner function at a later time $t$ can be approximated by a Gaussian distribution centered at the classical reference orbit passing through $\left(n_{\mathrm{cl}}(0), p_{\mathrm{cl}}(0)\right)$ with covariance

$$
C(t)=S(t) C(0) S(t)^{T} .
$$

Here $S(t)$ is the linear approximation, i.e. the Jacobian matrix, of the classical Hamiltonian flow and is thus a symplectic two by two matrix (see also Appendix D for further details). In other words, $S(t)$ is the fundamental solution of Hamilton's equations of motion linearized around the reference orbit and obeys the non-autonomous differential equation

$$
\dot{S}(t)=J H^{\prime \prime}\left(n_{\mathrm{cl}}(t), p_{\mathrm{cl}}(t)\right) S(t)
$$

with initial condition $S(0)=$ id. The nearby orbit approximation is due to Heller et al. and Littlejohn et al. [66-70] and was further developed e.g. in [71-74] (also see [53,54] for extensive reviews). A related, though different approximation is discussed in [75].

We stress that Eq. (8) involves two approximations. First, the full quantum dynamics is approximated by the classical Liouville equation of the Wigner function. And second, as the initial Wigner function is a strongly localized Gaussian in phase space, Liouville's equation is approximately solved by a Gaussian centered at the classical reference orbit within the nearby orbit approximation. As shown in Appendix C, Eqs. (7) and (8) are equivalent.

\section{Results for the variance}

We now discuss the dynamics of the expectation value of spin operators in the spin system (1) after a sudden quantum quench in the external magnetic field $\Gamma$. More specifically, we are interested in the dynamics of the magnetization per site $\left\langle n_{+}\right\rangle$and its variance. That is, we prepare the initial state as the ground state of the pre-quench Hamiltonian $\mathcal{H}\left(\Gamma_{i}\right)$ with an external magnetic field $\Gamma_{i}$ and evolve the state with the post-quench Hamiltonian $\mathcal{H}\left(\Gamma_{f}\right)$, where $\Gamma_{f}$ is different from $\Gamma_{i}$ such that the post-quench Hamiltonian does not commute with the pre-quench Hamiltonian and the dynamics is non-trivial. 
Preparing the state in the ground state of the pre-quench Hamiltonian fixes the initial conditions $n_{\mathrm{cl}}(0), p_{\mathrm{cl}}(0)$, and $f_{2}(0)$. As before, we denote the global minimum of $\Re f$ by $n_{\mathrm{cl}}$, and write $p_{\mathrm{cl}}$ for $i f^{\prime}\left(n_{\mathrm{cl}}\right)$ and $f_{n}$ for the Taylor coefficients $f^{(n)}\left(n_{\mathrm{cl}}\right)$. The ground state of the prequench Hamiltonian obeys the eigenvalue equation $H\left(n_{+},-i \partial_{n_{+}} / N\right) e^{-N f\left(n_{+}\right)}=E e^{-N f\left(n_{+}\right)}$ with ground state energy $E$. By neglecting zero-point fluctuations in the energy $E$, which are of order $1 / N$, we may write

$$
H\left(n_{+}, i f^{\prime}\right) \approx E
$$

instead of $\left[H\left(n_{+}, i f^{\prime}\right)+\mathcal{O}(1 / N)\right] e^{-N f\left(n_{+}\right)}=E e^{-N f\left(n_{+}\right)}$. Taking the first derivative of (10) w.r.t. $n_{+}$at $n_{\mathrm{cl}}$, yields $H^{(1,0)}+i f_{2} H^{(0,1)}=0$, which is solved by any critical point $\left(n_{\mathrm{cl}}, p_{\mathrm{cl}}\right)$ of $H$. Since we are interested in the ground state, we choose the absolute minimum (assuming it exists and is unique). Intuitively, the ground state Wigner function is only significantly different from zero in the neighborhood of the absolute minimum of $H$, which gives the main contribution to $E$. The fact that the Wigner function is only localized on a scale of $1 / \sqrt{N}$ in phase space, leads to additional (zero-point) contributions of order $1 / N$ to the energy. Taking the second derivative of $(10)$ at $n_{+}=n_{\mathrm{cl}}$ yields $H^{(2,0)}+2 i f_{2} H^{(1,1)}+\left(i f_{2}\right)^{2} H^{(0,2)}+i f_{3} H^{(0,1)}=0$. Using $H^{(0,1)}=0$, the last equation can be written in matrix form as $\left(1, i f_{2}\right) H^{\prime \prime}\left(1, i f_{2}\right)=0$, where $H^{\prime \prime}$ denotes the Hessian matrix evaluated at the critical point $\left(n_{\mathrm{cl}}, p_{\mathrm{cl}}\right)$. This quadratic equation in $f_{2}$ and can be readily solved.

The initial condition are thus determined by

$$
\begin{aligned}
& n_{\mathrm{cl}}(0)= \begin{cases}\left( \pm \sqrt{1-4 \Gamma_{i}^{2}}+1\right) / 2, & \Gamma_{i}<1 / 2 \\
1 / 2, & \Gamma_{i}>1 / 2,\end{cases} \\
& p_{\mathrm{cl}}(0)=0, \\
& i f_{2}(0)=-\frac{H^{(1,1)}}{H^{0,2}} \pm \sqrt{\frac{H^{(1,1)}}{H^{0,2}}-\frac{H^{(2,0)}}{H^{(0,2)}}}
\end{aligned}
$$

( $H^{(n, m)}$ being the $n$th and $m$ th derivative of $H$ w.r.t. its first and second argument, respectively, evaluated at $\left.\left(n_{\mathrm{cl}}, p_{\mathrm{cl}}\right)\right)$. Equations (11a) and (11b) determine the absolute minimum of the Hamiltonian function $H$ [39]. The critical points of $H\left(n_{+}, 0\right)$ undergo a pitchfork bifurcation at the critical point $\Gamma_{c}=1 / 2$. In the ferromagnetic phase, for $\Gamma_{i}<\Gamma_{c}$, the symmetry under spin-flips leads to the two-fold degeneracy of the ground state in the thermodynamic limit. This is reflected by the fact that $H$ has two minima on equal footing. From now on, we tacitly assume that the spin-flip symmetry is broken, e.g. by adding the infinitesimal longitudinal field term $\epsilon S_{z}$ with $\epsilon=\mathcal{O}(1 / N)$ to the Hamiltonian (1), and thereby singling out the positive square root in (11a). Note, that (11a)-(11c) is a fixed point of the classical equations of motion and Eq. (7) for $\Gamma=\Gamma_{i}$, i.e. when no quench is done. However, for $\Gamma=\Gamma_{f} \neq \Gamma_{i}$ the dynamics is non-trivial (see Figs. 2 and 3).

Figure 1 depicts six particular qualitatively different quenches in a dynamical phase diagram as pairs of $\left(\Gamma_{i}, \Gamma_{f}\right)$. This dynamical phase diagram was discussed by Biroli and Sciolla in [39] in the context of dynamical phase transitions. Biroli et al. define a dynamical phase transition as a discontinuity of the late time behavior of the order parameter as a function of the quench parameter, also see [24-26]. In this section we complement the discussion with the dynamics of the variance $\operatorname{var}\left(n_{+}\right)$in Figs. 2 and 3. These results are valuable for the understanding of the entanglement dynamics in Sec. 5 .

Based on the qualitative behavior of the variance, we distinguish four different regimes in the dynamical phase diagram, as indicated by the Roman numerals in Fig. 1. 


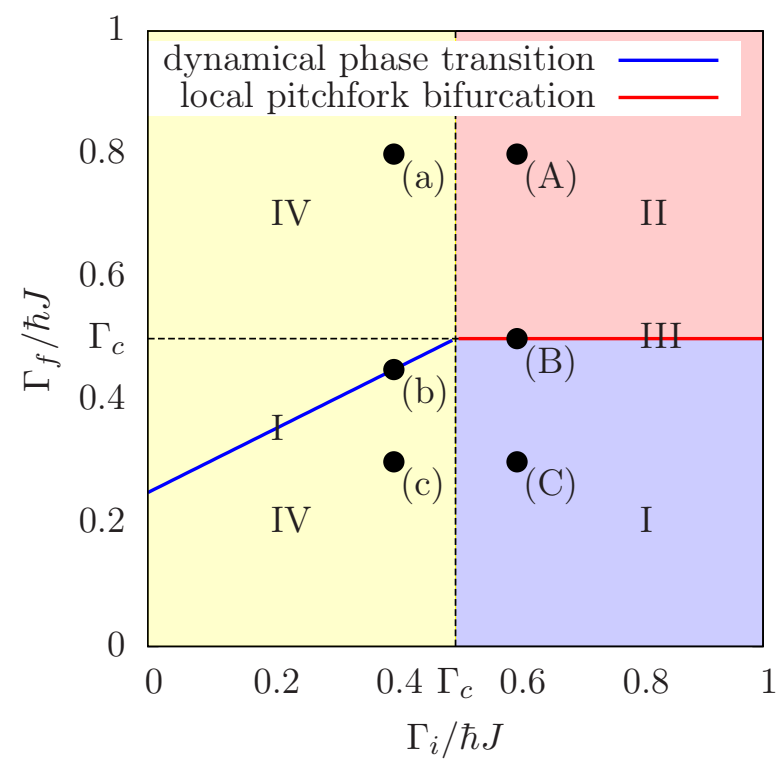

Figure 1: Dynamical phase diagram for the sudden quench $\Gamma_{i} \rightarrow \Gamma_{f}$ in the Hamiltonian (1) (compare Ref. [39]). Black dots with lower and upper case Latin letters indicate the quenches shown in Figs. 2 and 3, respectively. The different colors and Roman numerals indicate different qualitative behavior of the time evolution of the variance. Region I: exponential growth (cf. Fig. 2 (b) and Fig. 3 (C)); region II: periodic oscillations (cf. Fig. 3 (A)); region III: quadratic growth without squeezing (cf. Fig. 3 (B)); region IV: periodically enhanced squeezing and quadratic growth (cf. Figs. 2 (a) and (c)).
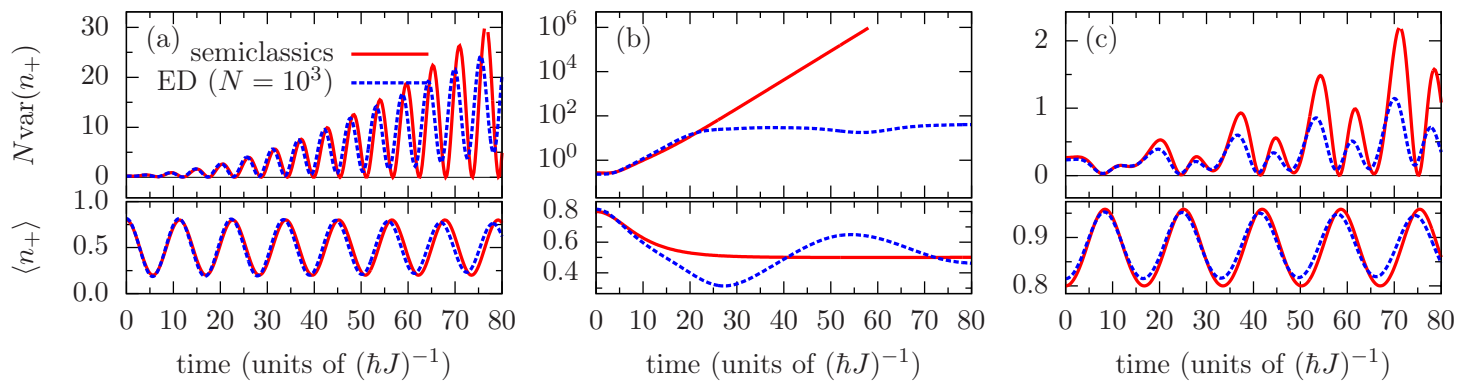

Figure 2: Dynamics of the spin expectation value $\left\langle n_{+}\right\rangle$(bottom) and its (rescaled) variance $N \operatorname{var}\left(n_{+}\right)$(top) after a sudden quantum quench from $\Gamma_{i}=0.4$ to $\Gamma_{f}=0.8$ (a), $\Gamma_{f}=$ 0.45 (b), and $\Gamma_{f}=0.3$ (c) (cf. Fig. 1). The results are obtained by exact diagonalization with $N=10^{3}$ (dotted blue line) and by a leading order semiclassical expansion $\lim _{N \rightarrow \infty}\left\langle n_{+}\right\rangle$and $\lim _{N \rightarrow \infty} N \operatorname{var}\left(n_{+}\right)$(solid red line) according to Eqs. (4) and (5). 

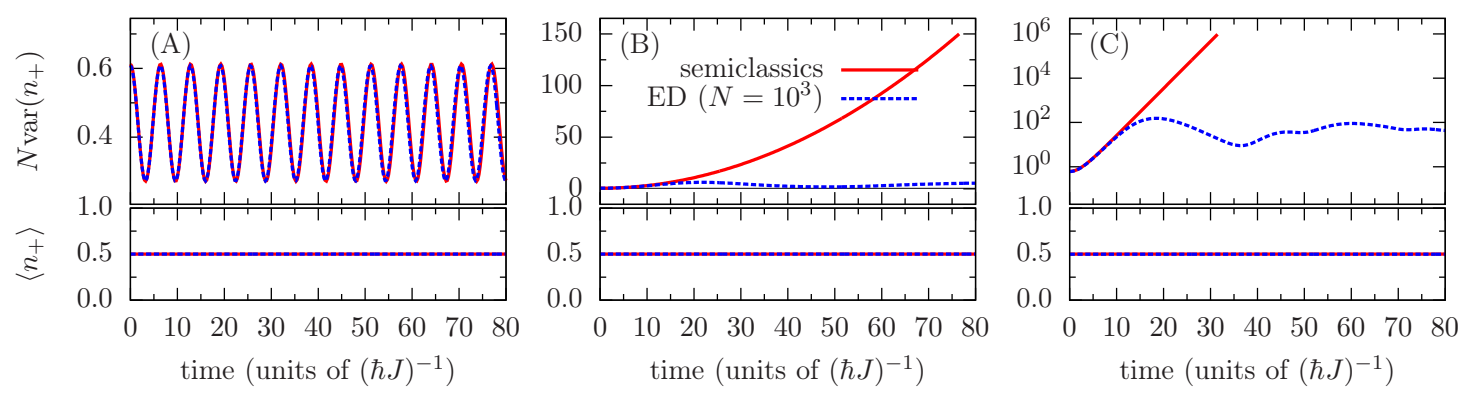

Figure 3: Dynamics of the spin expectation value $\left\langle n_{+}\right\rangle$(bottom) and its (rescaled) variance $N \operatorname{var}\left(n_{+}\right)$(top) after a sudden quantum quench from $\Gamma_{i}=0.6$ to $\Gamma_{f}=0.8(\mathrm{~A}), \Gamma_{f}=$ 0.5 (B), and $\Gamma_{f}=0.3$ (C) (cf. Fig. 1). The results are obtained by exact diagonalization with $N=10^{3}$ (dotted blue line) and by a leading order semiclassical expansion $\lim _{N \rightarrow \infty}\left\langle n_{+}\right\rangle$and $\lim _{N \rightarrow \infty} N \operatorname{var}\left(n_{+}\right)$(solid red line) according to Eqs. (4) and (5).

\subsection{Exponential growth regime (I)}

For quenches from the paramagnetic phase to the ferromagnetic phase (exemplified by the quench (C) in Fig. 3), as well as for quenches on the critical line of the dynamical phase transition (exemplified by the quench (b) in Fig. 2)), the variance starts to increases exponentially in time before it saturates and shows minor oscillations around a finite value. The saturation process is due to finite size effects and is not captured in the semiclassical result $\lim _{N \rightarrow \infty} N \operatorname{var}\left(n_{+}\right)$.

In the case of the quench in Fig. $3(\mathrm{C})$ the exponential increase can be readily understood from the fact that the initial wave packet is localized at the hyperbolic critical point $\left(n_{\mathrm{cl}}, p_{\mathrm{cl}}\right)=$ $(1 / 2,0)$ of the post-quench Hamiltonian, cf. Eq. (11a) [76]. From the point of view of Eq. (8) one can argue as follows. If $\lambda_{1}<0<\lambda_{2}$ denote the eigenvalues of the Hessian $H^{\prime \prime}\left(\Gamma_{f}\right)$ evaluated at the hyperbolic point, then the eigenvalues of $S(t)=\exp \left(J H^{\prime \prime}\left(\Gamma_{f}\right) t\right)$ are $e^{ \pm \omega t}$, where $\omega=\sqrt{\left|\lambda_{1} \lambda_{2}\right|}$. Hence, the covariance matrix $C(t)=S(t) C(0) S(t)^{T}$ has an exponentially increasing and an exponentially decreasing eigenvalue in time. For late times, the direction of decreasing variance becomes orthogonal to the stable manifold of the hyperbolic fixed point (a more detailed discussion can be found in Appendix E). For all other directions the exponentially increasing contribution eventually dominates the variance. In particular, $\operatorname{var}\left(n_{+}\right)=C_{11}(t)$ increases exponentially.

For quenches on the critical line (see Fig. 2 (b)), the mean of the initial Wigner distribution lies on a separatrix of the post-quench Hamiltonian. More precisely, the separatrix is a homoclinic orbit and connects the stable and unstable direction of the hyperbolic critical point $(1 / 2,0)$ of $H\left(\Gamma_{f}\right)$. As the mean of the Wigner function approaches the hyperbolic fixed point on the separatrix, the dynamics of its variance is dominated by the hyperbolic fixed point and increases exponentially, as discussed above.

\subsection{Periodic regime (II)}

For quenches within the paramagnetic phase (region II in Fig. 1) the post quench Hamiltonian has an elliptic fixed point at $(1 / 2,0)$, where the initial Wigner function is localized. 
Consequently, the eigenvalues of $S(t)$ are phase factors $e^{ \pm i \omega t}$, where $\omega=\sqrt{\left|\lambda_{1} \lambda_{2}\right|}$, and the covariance matrix $C(t)$ is $2 \pi / \omega$ periodic (see Fig. $3(\mathrm{~A})$ ). Note that the semiclassical result $\lim _{N \rightarrow \infty} N \operatorname{var}\left(n_{+}\right)$agrees with the exact diagonalization data for much later times than in regime (I). Essentially, this is because the Wigner function remains well localized also for late times, which is the key assumption for the validity of the nearby orbit approximation and the rate function expansion.

\subsection{Quadratic growth regime (III)}

The exponential regime (I) and the periodic regime (II) are separated by regime (III) in which the variance increases quadratically. For quenches on this line the initial Wigner function is centered at a degenerate fixed point of the critical post-quench Hamiltonian. The degeneracy leads to the fact that $S(t)$ is a shear matrix whose shear factor scales linearly with time (see Appendix E). Consequently, the eigenvalues of $C(t)$ scale quadratically and inversely quadratic in time. The associated eigenvectors approach the eigenvectors of $H^{\prime \prime}\left(\Gamma_{f}\right)$ (the eigenvalue zero eigenvector of the Hessian is approached by the quadratically increasing eigendirection of $C(t)$ ). Along any direction different from the eigendirection in which $C(t)$ decreases, the quadratically increasing contribution dominates for late times, such that the variance increases quadratically in those directions. In particular, $\operatorname{var}\left(n_{+}\right)=C_{11}(t)$ increases quadratically (see Fig. $3(\mathrm{~B})$ ).

Also note that regimes (I), (II) and (III) cannot be distinguished by just looking at the expectation value $\left\langle n_{+}\right\rangle$. Notwithstanding, its variance behaves qualitatively very different in each case.

\subsection{Periodically enhanced squeezing regime (IV)}

For quenches starting in the ferromagnetic phase and not lying on the critical line of the dynamical phase transition (region IV in Fig. 1), the expectation value oscillates coherently with period $T$. The variance shows quasi-periodic oscillations of the same period $T$ within the envelope of quadratically increasing and inversely quadratic decreasing bounds, Fig. 4. We refer to this behavior as periodically enhanced squeezing and periodically enhanced spreading. Among all regimes, this is the less intuitive and, to the authors' knowledge, has not been described in the literature so far. In contrast to the regimes (I), (II) and (III) the mechanism is not related to fixed point dynamics of the Hamiltonian flow and therefore genuinely different.

It turns out that the dichotomy of periodically enhanced squeezing and periodically enhanced spreading is the effect of a common cause. As elaborated in Appendix D, the periodicity of the reference orbit allows to apply Floquet's theorem to Eq. (9) and yields $S(t)=P(t) M(t)$ where $P(t)$ is a $T$-periodic two by two matrix and $M(t)$ is a shear matrix with shear factor proportional to time $t$. A non-harmonic Hamiltonian is a necessary condition for the shear factor to be different from zero (see Appendix D). Intuitively, a nonzero shear factor means that two nearby periodic orbits have different periods, which is the rule rather than the exception. An explicit expression of the shear factor is derived in Eqs. (38) and (39). Analogous to regime (III), the eigenvalues of $M(t) C(0) M(t)^{T}$ scale quadratically and inversely quadratic at late times. Let the corresponding eigenvectors be $|+\rangle(t)$ and $|-\rangle(t)$, respectively. For any fixed initial direction $|v\rangle,|w(t)\rangle=P(t)^{T}|v\rangle$ traverses all directions in the two-dimensional phase space at least once in each period, see Appendix D. As a consequence, $C_{v}(t)=\langle v|C(t)| v\rangle=\left\langle w(t)\left|M(t) C(0) M(t)^{T}\right| w(t)\right\rangle$ has a local minimum and maximum when- 


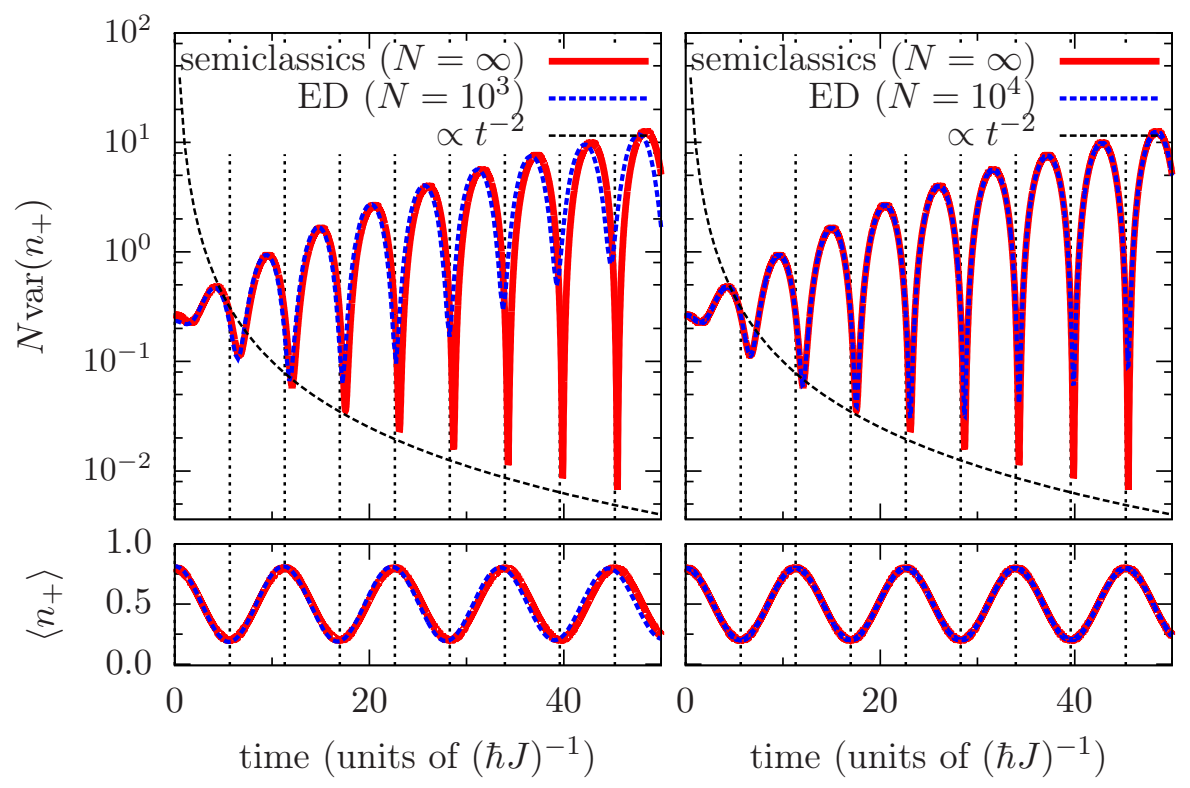

Figure 4: Same quench as in Fig. 2 (a) for $N=10^{3}$ (left) and $N=10^{4}$ (right). Periodically enhanced squeezing: In the semiclassical limit (solid red line) the minima of $\operatorname{var}\left(n_{+}\right)$decrease inversely quadratic with time (the dashed black line is a guide to the eye). The positions of the minima approach the turning points of the order parameter (vertical dotted black lines) for late times. The ED data (dashed blue line) agrees with the semiclassical result for early times. 
ever $|w(t)\rangle$ aligns with the vector $|-\rangle(t)$ and $|+\rangle(t)$, respectively. This results in the observed periodically enhances squeezing and spreading.

Interestingly, the details of the Hamiltonian do not matter, as long as the reference orbit is periodic and nearby orbits have different periods. In this sense, our observations are universal and to be found in other mean field models, which possess an effective semiclassical twodimensional phase space description such as the Bose-Hubbard model or the Jaynes-Cummings model on a fully connected lattice [39]. Also, the universality of the periodically enhanced spreading and squeezing shows in the fact that the variance dynamics is qualitatively identical on both sides of the dynamical phase transition, cf. Figs. 2 (a) and (c).

\subsection{Validity of the mean field approximation in non-equilibrium}

We comment on the validity of the semiclassical results. At some point in time, the semiclassical results start to deviate from the exact diagonalization data. A natural question is thus: Up to which timescale can one trust the semiclassical results? This question is really a question about the order of the two limits $N \rightarrow \infty$ and $t \rightarrow \infty$. If the limit $N \rightarrow \infty$ is taken first, the semiclassical results become exact for all times. However, we consider the situation when $N$ is huge but finite, and late times are probed for fixed $N$.

A necessary condition for the validity of the saddle point approximation, on which the semiclassical results (4) and (5) rely, is that $|\psi|^{2}$ in (3) remains localized on a scale of $1 / \sqrt{N}$. More precisely, the leading order saddle point approximation breaks down when the inverse curvature of the rate function at the saddle point is of the order of the saddle point parameter, i.e. $N$.

From the point of view of the nearby orbit approximation, mean field breaks down when the eigenvalues of the covariance matrix $C(t)=S(t) C(0) S(t)^{T}$ becomes large, such that orbits far away from the reference orbit need to be taken into account. For orbits far away from the reference orbit, the linear approximation of the equations of motion, on which the nearby orbit approximation relies, is inaccurate and errors accumulate. In other words, the nearby orbit approximation breaks down at the (Ehrenfest) timescale $t_{E}^{*}$ when the spread of the wave packet reaches the scale $\chi$, on which the Hamiltonian can only be badly approximated to quadratic order. A heuristic estimate of this length scale, motivated by a Moyal bracket expansion, is given by $\chi \sim \sqrt{\partial_{x} V(x) / \partial_{x}^{3} V(x)}$ [77]. To get the scaling exponent of $t_{E}^{*}$ as a function of system size, the order of magnitude of $\chi$ is not crucial. Indeed, for polynomial growth, $\sqrt{\operatorname{var}} \sim t^{\alpha} / \sqrt{N}$, the condition $\sqrt{\operatorname{var}} \lesssim \chi$ implies $t_{E}^{*} \sim N^{1 /(2 \alpha)}$, and for exponential growth, $\sqrt{\operatorname{var}} \sim e^{\lambda t} / \sqrt{N}$, one gets $t_{E}^{*} \sim \log N$.

Concerning the different regimes of Fig. 1, we conclude that the semiclassical dynamics is only valid up to short timescales of order $\log N$ for quenches in regime (I) and to times of order $\sqrt{N}$ in regimes (III) and (IV). In regime (IV), in which the order parameter evolves on a periodic orbit, the effect of anharmonic terms in the Hamiltonian is twofold. First, anharmonic terms in the Hamiltonian inevitably cause the wave packet to spread, and squeeze within quadratically increasing, and inversely quadratic decreasing bounds. Second, as the variance of the wave packet becomes of the order of $\chi$, the anharmonic terms cause the breakdown of the mean field approximation.

We emphasize these findings. Even in fully connected lattice model, for which one believes mean field models to yield reliable results, the out of equilibrium mean field dynamics can already start to break down on a relatively short timescale of order $\sqrt{N}$ (regimes III and IV), and even $\log N$ (regime I). 

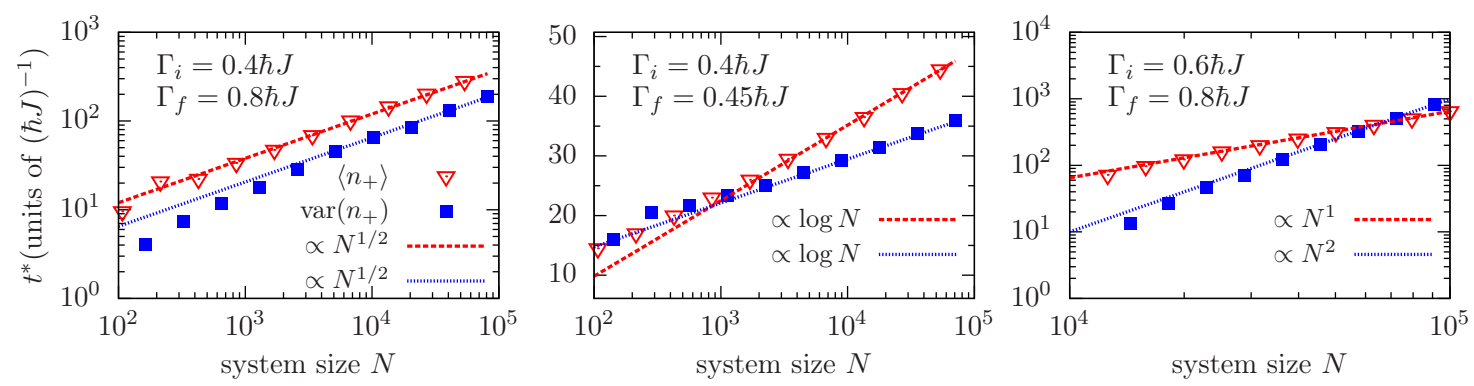

Figure 5: Timescale of validity for the mean field approximation as a function of system size $N$. First instant of time $t^{*}$, when the leading order correction to the mean field order parameter $\left\langle n_{+}\right\rangle$(red open triangles), cf. Eq. (4a), and the mean field variance $\operatorname{var}\left(n_{+}\right)$(blue filled squares), cf. Eq. (5a), exceeds a fixed, but arbitrarily chosen threshold. The dashed red and dotted blue lines are guides to the eye. Square root scaling, $t^{*} \propto \sqrt{N}$, for quench (a) in regime IV (left), logarithmic scaling, $t^{*} \propto \log N$, for quench (b) in regime I (middle), linear scaling, $t^{*} \propto N$, for quench (A) in regime II (right), cf. Fig. 1. Note, the left and right plots are double-logarithmic, the middle plot is semi-logarithmic.

To confirm this heuristic intuition numerically, we investigate the first time instant $t^{*}$ at which the leading order correction to the expectation and the variance of the order parameter becomes larger than a arbitrary and fixed threshold, see Fig. 5. These correction terms are functions of $\Re f_{3}$ and $\Re f_{4}$, cf. Eq. (32), whose evolution via (31) are sensitive to anharmonic terms of the Hamiltonian.

Quenches within the paramagnetic phase (regime II), where the wave packet is centered at a stable fixed point, are special for two reasons. First, due to spin-flip symmetry $n_{+} \mapsto$ $\left(1-n_{+}\right)$, the expectation value $\left\langle n_{+}\right\rangle=1 / 2$ predicted by mean field is 'accidentally' exact, independent of the system size $N$, and for all times. Second, the evolution of the variance to leading order as given by Eq. (7), depends only on the harmonic part of the Hamiltonian, and is bounded for all times. Despite these facts, one cannot trust the mean field predictions to arbitrarily late times. This becomes apparent, when corrections to the variance are considered, which become significant in size at time $t^{*} \sim N^{2}$, see Fig. 5. Spin-flip symmetry implies that all corrections to the mean field limit of $\left\langle n_{+}\right\rangle$vanish exactly. To probe the validity of the mean field result for practical purposes, we break the symmetry by adding a term $\epsilon S_{z}$ to the post-quench Hamiltonian with an infinitesimal longitudinal field $\epsilon$. Then, correction terms to the expectation value build up to a non-negligible contribution on timescales of $t^{*} \sim N$ being linear in system size, see Fig. 5. The quadratic scaling $t^{*} \sim N^{2}$ for the variance corrections is not affected by the symmetry breaking.

\section{$5 \quad$ Entanglement dynamics}

The ground state entanglement entropy of the fully connected transverse field Ising model has been computed numerically for finite system sizes [78] and analytically in the thermodynamic limit [79] by applying the Holstein-Primakoff [80] transformation and expanding the Hamiltonian in the reciprocal system size. One of the motivations to study the ground state entanglement entropy is its scaling behavior at quantum critical points [81,82]. A change in 
scaling of the mutual information at criticality has also been observed for non-zero temperature thermal density matrices [83].

Entanglement dynamics has been investigated in long-range models with power law interaction, such as harmonic oscillator chains [84], fermionic hopping models [27], spin models $[18,28,36,37]$, and disordered models [19]. Entanglement dynamics as measured by the one-tangle and the concurrence has been investigated in [36] for the fully connected transverse field Ising model.

In the literature so far, the entanglement dynamics has been investigated mainly for fully polarized initial conditions $[18,27,36]$. Since we are ultimately interested in the entanglement entropy of time evolved pre-quench ground states, we follow a different, though related, approach. We will systematically discuss the entanglement dynamics in the dynamical phase diagram of the sudden quench setup. One advantage is that the quantitative connection between entanglement and spin squeezing is apparent in our approach.

\subsection{Bipartition and reduced density matrix}

We want to compute the bipartite entanglement entropy relative to the bipartition $\mathcal{H}_{N}=$ $\mathcal{H}_{N_{A}} \otimes \mathcal{H}_{N_{B}}$. That is, we divide the set of $N=N_{A}+N_{B}$ spins into two disjoint sets containing $N_{A}$ and $N_{B}$ spins, respectively. Due to the fully connected geometry, the particular choice of the separation into $A$ and $B$ is arbitrary. But once a choice is made, it is fixed over the course of time. Each of the two factors, $\mathcal{H}_{N_{A}}$ and $\mathcal{H}_{N_{B}}$, contains a $\left(N_{A}+1\right)$ and $\left(N_{B}+1\right)$-dimensional permutation invariant Dicke subspace, respectively. The state $\left|N_{+}\right\rangle$is expanded in the Dicke basis of the subsystems $A$ and $B$ as

$$
\left|N_{+}\right\rangle=\sum_{A_{+}+B_{+}=N_{+}} \sqrt{\left(\begin{array}{c}
N_{A} \\
A_{+}
\end{array}\right)\left(\begin{array}{c}
N_{B} \\
B_{+}
\end{array}\right) /\left(\begin{array}{c}
N \\
N_{+}
\end{array}\right)}\left|A_{+}\right\rangle\left|B_{+}\right\rangle .
$$

The summation is over all nonnegative integers $0 \leq A_{+} \leq N_{A}$ and $0 \leq B_{+} \leq N_{B}$ obeying the constraint $A_{+}+B_{+}=N_{+}$. The decomposition is unique. Essentially, the combinatorial factor

$$
\sqrt{\left(\begin{array}{c}
N_{A} \\
A_{+}
\end{array}\right)\left(\begin{array}{c}
N_{B} \\
B_{+}
\end{array}\right) /\left(\begin{array}{c}
N \\
N_{+}
\end{array}\right)}
$$

reflects the fact that there are more ways to permute $N_{+}=A_{+}+B_{+}$up-spins among $N=$ $N_{A}+N_{B}$ spins than to independently permute $A_{+}$and $B_{+}$up-spins among $N_{A}$ and $N_{B}$ spins, respectively.

We want to prove Eq. (12). How does the permutation invariant state $\left|N_{+}\right\rangle$split into the two permutation invariant subsystems? Equation (12) follows from the identity

$$
\begin{array}{rl}
\left(\begin{array}{c}
N \\
N_{+}
\end{array}\right) P & P\left(|\uparrow\rangle^{\otimes N_{+}} \otimes|\downarrow\rangle^{\otimes N-N_{+}}\right) \\
& =\left(|\uparrow\rangle^{\otimes N_{+}} \otimes|\downarrow\rangle^{\otimes N-N_{+}}+\text {proper perm. }\right) \\
& =\sum_{A_{+}+B_{+}=N_{+}}\left(|\uparrow\rangle^{\otimes A_{+}} \otimes|\downarrow\rangle^{\otimes N_{A}-A_{+}}+\text {proper perm. }\right)\left(|\uparrow\rangle^{\otimes B_{+}} \otimes|\downarrow\rangle^{\otimes N_{B}-B_{+}}+\text {proper perm. }\right) \\
& =\sum_{A_{+}+B_{+}=N_{+}}\left(\begin{array}{c}
N_{A} \\
A_{+}
\end{array}\right) P\left(|\uparrow\rangle^{\otimes A_{+}} \otimes|\downarrow\rangle^{\otimes N_{A}-A_{+}}\right)\left(\begin{array}{c}
N_{B} \\
B_{+}
\end{array}\right) P\left(|\uparrow\rangle^{\otimes B_{+}} \otimes|\downarrow\rangle^{\otimes N_{B}-B_{+}}\right)
\end{array}
$$


(by proper permutation we mean only those permutations that lead to different spin configurations, e.g. permutations that permute only up-spins are not included). Thus,

$$
\begin{aligned}
\left|N_{+}\right\rangle & =\left(\begin{array}{c}
N \\
N_{+}
\end{array}\right)^{1 / 2} P\left(|\uparrow\rangle^{\otimes N_{+}} \otimes|\downarrow\rangle^{\otimes N-N_{+}}\right) \\
& =\sum_{A_{+}+B_{+}=N_{+}} \sqrt{\left(\begin{array}{c}
N_{A} \\
A_{+}
\end{array}\right)\left(\begin{array}{c}
N_{B} \\
B_{+}
\end{array}\right) /\left(\begin{array}{c}
N \\
N_{+}
\end{array}\right)}\left|A_{+}\right\rangle\left|B_{+}\right\rangle .
\end{aligned}
$$

A generic pure state in $\mathcal{D}_{N}$ is the superposition $|\Psi\rangle=\sum_{N_{+}} \psi\left(N_{+}\right)\left|N_{+}\right\rangle$, and the corresponding density matrix is $\rho\left(N_{+} ; \tilde{N}_{+}\right)=\psi\left(N_{+}\right) \psi^{*}\left(\tilde{N}_{+}\right)$. We can also expand $|\Psi\rangle$ in the Dicke basis of the bipartite system as $|\Psi\rangle=\sum_{A_{+}, B_{+}} \psi_{A B}\left(A_{+}, B_{+}\right)\left|A_{+}\right\rangle\left|B_{+}\right\rangle$, where

$$
\psi_{A B}\left(A_{+}, B_{+}\right)=\psi\left(A_{+}+B_{+}\right) \sqrt{\left(\begin{array}{l}
N_{A} \\
A_{+}
\end{array}\right)\left(\begin{array}{l}
N_{B} \\
B_{+}
\end{array}\right) /\left(\begin{array}{c}
N \\
A_{+}+B_{+}
\end{array}\right)}
$$

follows from Eq. (12). To shorten the notation, we will sometimes write $\psi$ for the coefficient $\psi_{A B}$ of the composite system and distinguish it from the other $\psi$ by the number of arguments. In general, the right hand side of (14) does not factorize into a product of functions depending solely on $A_{+}$respectively $B_{+}$. This shows that the state is entangled. The density matrix associated to $\psi_{A B}$ is $\rho_{A B}\left(A_{+}, B_{+} ; \tilde{A}_{+}, \tilde{B}_{+}\right)=\psi\left(A_{+}, B_{+}\right) \psi^{*}\left(\tilde{A}_{+}, \tilde{B}_{+}\right)$and the reduced density matrix of subsystem $A$ is $\rho_{A}\left(A_{+}, \tilde{A}_{+}\right)=\sum_{B_{+}} \rho_{A B}\left(A_{+}, B_{+} ; \tilde{A}_{+}, B_{+}\right)$.

The expectation value of the magnetization per spin in each subsystem agrees with the magnetization per spin of the total system. That is,

$$
\operatorname{Tr}\left(\rho_{A} A_{+}\right) / N_{A}=\left\langle n_{+}\right\rangle .
$$

This is an exact result and follows readily from Eq. (14) and the Vandermonde identity,

$$
\begin{aligned}
\left\langle A_{+} / N_{A}\right\rangle & =\sum_{A_{+}, B_{+}}\left|\psi\left(A_{+}, B_{+}\right)\right|^{2} A_{+} / N_{A} \\
& =\sum_{A_{+}, B_{+}}\left|\psi\left(A_{+}+B_{+}\right)\right|^{2} \frac{A_{+}}{N_{A}}\left(\begin{array}{c}
N_{A} \\
A_{+}
\end{array}\right)\left(\begin{array}{c}
N_{B} \\
B_{+}
\end{array}\right) /\left(\begin{array}{c}
N \\
A_{+}+B_{+}
\end{array}\right) \\
& =\sum_{N^{+}}\left|\psi\left(N^{+}\right)\right|^{2}\left(\begin{array}{c}
N \\
N^{+}
\end{array}\right)^{-1} \sum_{A_{+}}\left(\begin{array}{c}
N_{A}-1 \\
A_{+}-1
\end{array}\right)\left(\begin{array}{c}
N_{B} \\
N^{+}-A_{+}
\end{array}\right) \\
& =\sum_{N^{+}}\left|\psi\left(N^{+}\right)\right|^{2}\left(\begin{array}{c}
N \\
N^{+}
\end{array}\right)^{-1}\left(\begin{array}{c}
N-1 \\
N^{+}-1
\end{array}\right) \\
& =\sum_{N^{+}}\left|\psi\left(N^{+}\right)\right|^{2} N^{+} / N=\left\langle N^{+} / N\right\rangle .
\end{aligned}
$$

Eq. (15) is no longer true for higher moments, e.g. in general $\operatorname{Tr}\left(\rho_{A} A_{+}^{2}\right) / N_{A} \neq\left\langle n_{+}^{2}\right\rangle$, see Eq. (17).

The discussion so far, is valid for generic states in the Dicke subspace. In the remainder of this paragraph we concentrate on states of large deviation form. In particular, we derive the rate function of the reduced density matrix of the pure state (3). Using Eq. (3) in (14) 
yields that $\psi_{A B}$ is also of large deviation form $\psi_{A B}\left(A_{+}, B_{+}\right) \asymp \exp \left[-N f_{A B}\left(a_{+}, b_{+}\right)\right]$with rate function

$$
f_{A B}\left(a_{+}, b_{+}\right)=f\left(\alpha a_{+}+\beta b_{+}\right)+S_{\alpha \beta}\left(a_{+}, b_{+}\right) / 2 .
$$

Here, and in the sequel, small letters refer to percental quantities, such as the relative subsystem sizes $\alpha=N_{A} / N$ and $\beta=N_{B} / N$, and the fraction of up-spins $a_{+}=A_{+} / N_{A}$ and $b_{+}=B_{+} / N_{B}$ in subsystem $A$ and $B$, respectively. The multiplicative combinatorial factor (13) translates to the additive entropic contribution $S_{\alpha \beta}$ in (16). It follows readily from Stirling's formula that $S_{\alpha \beta}\left(a_{+}, b_{+}\right)=H_{2}\left(\alpha a_{+}+\beta b_{+}\right)-\alpha H_{2}\left(a_{+}\right)-\beta H_{2}\left(b_{+}\right)$, where $H_{2}(x)=-x \log x-(1-x) \log (1-x)$ is the classical binary Shannon entropy. Due to the concavity of the Shannon entropy, $S_{\alpha \beta}\left(a_{+}, b_{+}\right)$is non-negative and vanishes if and only if $a_{+}$and $b_{+}$are equal. In other words, fluctuations leading to $a_{+} \neq b_{+}$are exponentially suppressed. This plays a crucial role in the computation of the reduced density matrix. The term $S_{\alpha \beta}$ has an instructive interpretation. It is the classical information per spin that a demon acquires when splitting $N=N_{A}+N_{B}$ spins, containing exactly $N_{+}=A_{+}+B_{+}$up-spins, into two disjoint sets of $N_{A}$ and $N_{B}$ spins, each containing $A_{+}$and $B_{+}$up-spins, respectively. When the demon is blindfolded, the splitting is unbiased and $a_{+}=b_{+}=n_{+}$. No information is acquired in this case and $S_{\alpha \beta}\left(n_{+}, n_{+}\right)=0$.

Assuming, as before, that $\Re f\left(n_{+}\right)$has a unique global minimum at $n_{\mathrm{cl}}$, it follows from the properties of $S_{\alpha \beta}$ that $\Re f_{A B}\left(a_{+}, b_{+}\right)$has a unique global minimum at $a_{+}=b_{+}=n_{\mathrm{cl}}$. This is a manifestation of Eq. (15). We expand the composite rate function $f_{A B}$ around this minimum to second order. In this approximation $\psi_{A B}$ is a Gaussian wave function with inverse covariance matrix $N \Gamma^{A B}$,

$$
\Gamma^{A B}=f_{2}\left(\begin{array}{cc}
\alpha^{2} & \alpha \beta \\
\alpha \beta & \beta^{2}
\end{array}\right)+\frac{1}{2} \frac{1}{n_{\mathrm{cl}}\left(1-n_{\mathrm{cl}}\right)}\left(\begin{array}{cc}
\alpha \beta & -\alpha \beta \\
-\alpha \beta & \alpha \beta
\end{array}\right) .
$$

The latter term is the Hessian matrix of $S_{\alpha \beta} / 2$. For future reference, we define $S^{*}=$ $\alpha \beta /\left(n_{\mathrm{cl}}\left(1-n_{\mathrm{cl}}\right)\right)$.

To leading order in $1 / N$, the kernel of the reduced density matrix $\rho_{A}$ is again Gaussian and its inverse covariance $\Gamma^{A}$ is a function of $\Gamma^{A B}$ (see Eq. (48) in Appendix G for details), which yields the variance

$$
\begin{aligned}
& \operatorname{var}\left(a_{+}\right)=\frac{1}{2 N}\left(\Re f_{2}\right)^{-1}+\frac{1}{N} \frac{\beta^{2}}{S^{*}}+\mathcal{O}\left(N^{-2}\right), \\
& \operatorname{var}\left(p_{A}\right)=\frac{\alpha^{2}}{2 N}\left(\Re\left(f_{2}^{-1}\right)\right)^{-1}+\frac{1}{4 N} S^{*}+\mathcal{O}\left(N^{-2}\right),
\end{aligned}
$$

and covariance $\operatorname{var}(A, B):=\frac{1}{2}\langle A B+B A\rangle-\langle A\rangle\langle B\rangle$,

$$
\operatorname{var}\left(a_{+}, p_{A}\right)=\frac{\alpha}{N} \frac{\Im\left(f_{2}\right)}{\Re\left(f_{2}\right)}+\mathcal{O}\left(N^{-2}\right)
$$

of $a_{+}$and its conjugate momentum operator $p_{A}$ by a saddle point approximation. Eqs. (17) should be compared to Eqs. (5). Furthermore, the Wigner function

$$
W_{A}(z) \propto\left[-\frac{N}{2} z\left(\Sigma^{A}\right)^{-1} z\right]
$$

of $\rho_{A}$ is a Gaussian function of phase space coordinates $z=\left(a_{+}, p_{A}\right)$, and the two by two covariance matrix $\Sigma^{A}$ is $N$ independent with $\Sigma_{11}^{A} / N, \Sigma_{22}^{A} / N$, and $\Sigma_{12}^{A} / N=\Sigma_{21}^{A} / N$ given by (17a), (17b), and (17c), respectively. Details are presented in Appendix F. 


\subsection{Entanglement Hamiltonian}

Now, we compute the entanglement Hamiltonian $\widehat{H}_{E}$ w.r.t. the bipartition described above, i.e. we determine the operator $\widehat{H}_{E}$, such that $\rho_{A}=\exp \left(-\widehat{H}_{E}\right)$. Note that the Wigner function of $\exp \left(-\widehat{H}_{E}\right)$ is the Gaussian (18). However, we cannot immediately infer that the Wigner function of $\widehat{H}_{E}$ is the exponent $\frac{1}{2} z\left(\Sigma^{A}\right)^{-1} z$ of $W_{A}$, because, in general, the Wigner transform and the exponential do not commute (unless the exponent is a linear function in position and momentum). The correct way, to obtain the Wigner function $H_{E}(z)$ of $\widehat{H}_{E}$ from $W_{A}$, is to compute the star-exponential $\left[\exp ^{*}\left(-H_{E}\right)\right](z):=\sum_{n=0}\left(-H_{E}\right)^{* n}(z) / n$ ! of $H_{E}$, where $f^{* n}(z)$ denotes the Moyal star product of $n$ factors of $f(z)$, and match the result with $W_{A}=\exp ^{*}\left(-H_{E}\right)$. For a quadratic function $H_{E}=\frac{1}{2} z V z$ the star-exponential has been worked out in [85] as $\exp ^{*}\left(-H_{E}\right) \propto \exp \left[-\frac{1}{2} \frac{2}{\sqrt{\operatorname{det} V}} \tanh (\sqrt{\operatorname{det} V} / 2) z V z\right]$. We conclude that the entanglement Hamiltonian

$$
\widehat{H}_{E}=\frac{1}{2} z V z+\text { const }
$$

is quadratic, and the two by two matrix

$$
V=2 \sqrt{\operatorname{det} \Sigma^{A}} \operatorname{arctanh}\left[\left(2 \sqrt{\operatorname{det} \Sigma^{A}}\right)^{-1}\right]\left(\Sigma^{A}\right)^{-1}
$$

is proportional to the inverse covariance matrix of $\rho_{A}$. The additive constant results from the multiplicative normalization factor in (18), and can be determined a posteriori by the normalization condition $\operatorname{Tr} \rho_{A}=1$. It is interesting that in the semiclassical limit the entanglement Hamiltonian of collective spin states takes the simple form of a quantum harmonic oscillator. This is one of the rare cases, when the entanglement Hamiltonian can be computed explicitly.

Next, we compute the entanglement spectrum of $\rho_{A}$, equivalently, the spectrum of the harmonic oscillator $\widehat{H}_{E}$. According to Williamson's theorem, there exists a symplectic matrix $S \in \operatorname{Sp}(2)$ such that $S^{T} V S=\operatorname{diag}(\omega, \omega)$ is diagonal, and $\omega$ is the (unique) symplectic eigenvalue of $V$. Employing this canonical change of coordinates, transforms the entanglement Hamiltonian into the canonical form $\widehat{H}_{E}=\frac{\omega}{2} \widehat{S}\left(\widehat{a}_{+}^{2}+\widehat{p}_{A}^{2}\right) \widehat{S}^{\dagger}$, where $\widehat{S}$ is a metaplectic operator associated to the symplectic matrix $S$. Since the metaplectic operator is unitary, the spectrum is invariant under this transformation, and

$$
\operatorname{Spec}\left(\widehat{H}_{E}\right)=\text { const }+\mathbb{N}_{0} \omega .
$$

The additive constant combines the zero point energy and the constant in (19a). By Eq. (19b), $\omega$ is related to the symplectic eigenvalue $\lambda$ of $\Sigma^{A}$ via

$$
\omega=2 \operatorname{arctanh}[1 /(2 \lambda)] .
$$

Note that $\lambda$ is bounded from below by one half as a consequence of the uncertainty principle, see chapter 13 in [86], so that the argument of the arctanh function is always smaller than or equal to one.

In summary, the entanglement spectrum is equidistant, and a function of the symplectic eigenvalue of the covariance matrix of $\rho_{A}$. This can be viewed as a refinement of spin squeezing. Spin squeezing subsumes a collection of results around the generic idea that squeezed collective spin states, i.e. states for which the variance of the magnetization in a certain direction is below 
the standard quantum limit, are correlated among their elementary spins. These correlations show up in the entanglement of the state w.r.t. a bipartition of the set of elementary spins. Eq. (20) shows that in the large $N$ limit the effect of squeezing, as being measured by the symplectic eigenvalue of the covariance matrix, entails the full entanglement spectrum. To the author's knowledge, this result goes beyond common formulations of spin squeezing. In the following section we discuss the entanglement more closely by investigating the Rényi entanglement entropies.

\subsection{Rényi entanglement entropies}

The $n$th Rényi entanglement entropy $S_{A}^{(n)}=\left[\log \operatorname{Tr}\left(\rho_{A}^{n}\right)\right] /(1-n)$ follows from the entanglement spectrum $(20)$ and $e^{-\omega}=(2 \lambda-1) /(2 \lambda+1)=: \xi$,

$$
\begin{aligned}
S_{A}^{(n)} & =\frac{1}{n-1} \log \left[(\lambda+1 / 2)^{n}-(\lambda-1 / 2)^{n}\right] \\
& =\frac{1}{1-n} \log \frac{(1-\xi)^{n}}{1-\xi^{n}} .
\end{aligned}
$$

In particular, for $n \rightarrow 1$, the von Neumann entanglement entropy is

$$
\begin{aligned}
S_{A} & =\left(\lambda+\frac{1}{2}\right) \log \left(\lambda+\frac{1}{2}\right)-\left(\lambda-\frac{1}{2}\right) \log \left(\lambda-\frac{1}{2}\right) \\
& =H_{2}(\xi) /(1-\xi) .
\end{aligned}
$$

The fact that the von Neumann entropy of a Gaussian density matrix $\rho$ depends only on the symplectic spectrum of the covariance matrix of the Wigner function $W_{\rho}$, was already noted in [87]. Furthermore, the von Neumann entanglement entropy in fully connected spin models has been obtained by the two-boson formalism in [33]. Our result for the general Rényi entropies in the von Neumann limit is consistent with both of these results. Finally, let us remark that we have computed the Rényi entropies (22) by means of a replica calculation, see Appendix G, yielding the same result and providing yet another consistency check.

More explicitly, $\lambda=\sqrt{\operatorname{det} \Sigma^{A}}$ follows from Eqs. (17),

$$
\lambda=\sqrt{\frac{1}{4}+\frac{S^{*}}{4}\left[\frac{1}{2 \Re f_{2}}+\frac{1}{2 \Re\left(f_{2}^{-1}\right)}\left(\frac{2 \alpha \beta}{S^{*}}\right)^{2}-\frac{2 \alpha \beta}{S^{*}}\right]} .
$$

The fact that this expression contains the variance of $n_{+}$and $p$, cf. Eqs. (5), hints to the connection of spin squeezing. This connection is made more explicit below. Remarkably, $\lambda$ and therefore $S_{A}$ is independent of $N$. This is in contrast to the leading order term of the variance, which decreases as $1 / N$. As $N$ increases the wave function becomes more and more concentrated around the classical orbit $n_{\mathrm{cl}}$ in the effective picture, and the expectation value of a permutation invariant observable, such as the mean magnetization per site, is dominated by a single orbit. Quantum fluctuations around the expectation value as measured by the variance decrease and vanish in the limit $N \rightarrow \infty$. Nevertheless, the bipartite entanglement entropy, a pure quantum effect, saturates and reaches a non-zero plateau (compare Fig. 6) in the limit $N \rightarrow \infty$.

The entanglement entropy is a basis independent quantity that makes only reference to the splitting of the total Hilbert space and is independent of the basis choice in each tensor 


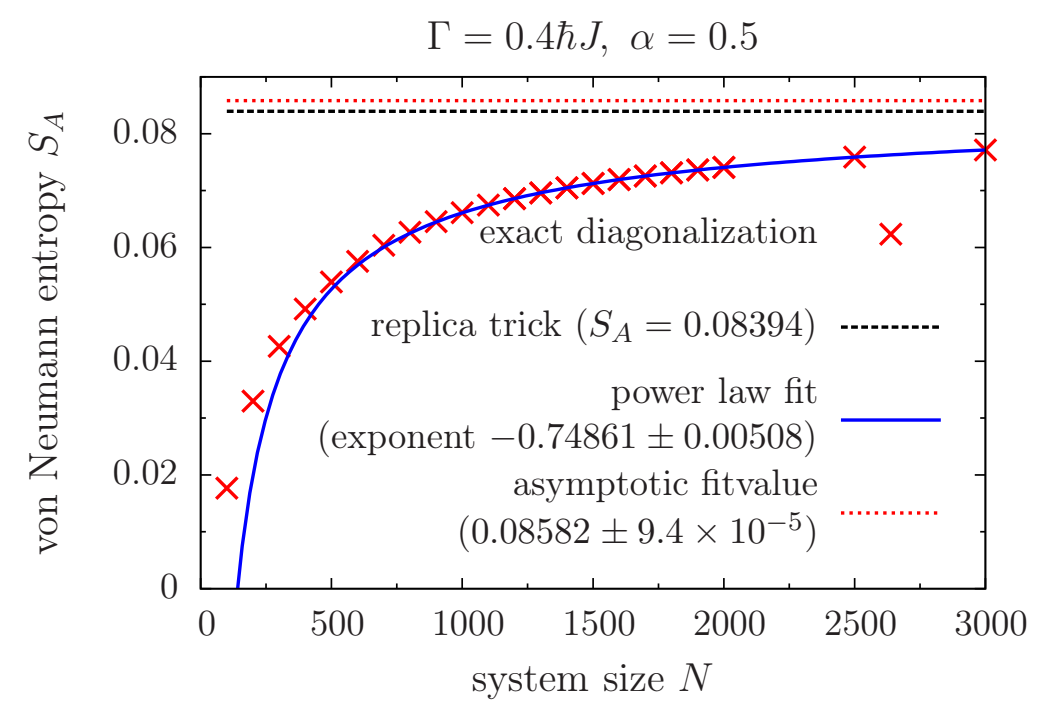

Figure 6: Bipartite entanglement entropy obtained by exact diagonalization as a function of system size (red crosses). In the limit $N \rightarrow \infty$ the entanglement entropy saturates to the result given by Eq. (23) (black dashed line), which agrees well with the asymptotic value (red dotted line) of the finite size fit of the exact diagonalization data (blue solid line).

factor. The calculation of $S_{A}$ in Eq. (23) is done in the eigenbasis of the spin in $z$-direction and leads to the fact that $\lambda$ depends on $n_{\mathrm{cl}}$ and $f_{2}$, which are not basis independent quantities. As a consequence, the form of Eq. (23) seems to single out a basis. However, this dependence is only an artifact of the representation as we will see below. We seek a more 'covariant' representation of $\lambda$ that is clearly invariant under rotation of the Bloch sphere. It turns out that $\lambda$ is a function of the basis independent spin squeezing parameter $\xi_{S}^{2}$ defined below.

One of the first references to establish the connection between entanglement and spin squeezing is the seminal paper of Kitagawa and Ueda [45], also see [46-48]. We review the qualitative argument of Ref. [45] why spin squeezing leads to entanglement. A spin $N / 2$ coherent spin state can be viewed a direct product of $N$ identical spin $1 / 2$ states. Coherent spin states may be considered to be 'most classical states' in the following sense. First, by construction, the individual $1 / 2$ spins of a coherent spin state are non-entangled among each other. And second, the variance of the magnetization is equally distributed among all directions perpendicular to the mean magnetization, such that the uncertainty (i.e. the product of the variance along any two orthogonal directions perpendicular to the mean) is minimal. The variance perpendicular to the mean magnetization in a coherent state is referred to as the standard quantum limit (SQL) [88]. Now, a spin state is said to be squeezed if there exists a direction normal to the mean magnetization along which the variance is below the standard quantum limit. In order to lower the variance below the standard quantum limit, correlations among the individual spins need to build up and the individual spins become entangled.

There is a multitude of spin squeezing measures [49]. Among them is what we refer to as the spin squeezing parameter $\xi_{S}^{2}$ being the ratio of the minimal to the maximal spin variance 
measured along directions perpendicular to the spin expectation value. More specifically, let $\hat{\Omega}=(\cos \phi \sin \theta, \sin \phi \sin \theta, \cos \theta)$ be the direction of the average spin on the Bloch sphere, i.e. $\langle\mathbf{S}\rangle=\hat{\Omega} / 2+\mathcal{O}(1 / N)$. We define two directions

$$
\begin{aligned}
& \hat{\Omega}_{1}^{\perp}=(-\sin \phi, \cos \phi, 0), \text { and } \\
& \hat{\Omega}_{2}^{\perp}=(-\cos \phi \cos \theta,-\sin \phi \cos \theta, \sin \theta)
\end{aligned}
$$

perpendicular to $\hat{\Omega}$. The covariance matrix of the spin in the subspace spanned by $\hat{\Omega}_{1}^{\perp}$ and $\hat{\Omega}_{2}^{\perp}$ is given by

$$
C_{i j}^{\perp}:=\left\langle S_{i}^{\perp} S_{j}^{\perp}\right\rangle-\left\langle S_{i}^{\perp}\right\rangle\left\langle S_{j}^{\perp}\right\rangle,
$$

where $S_{i}^{\perp}=\mathbf{S} \cdot \hat{\Omega}_{i}^{\perp}$. The spin squeezing parameter is then defined as

$$
\xi_{S}^{2}=\frac{\min _{\hat{\Omega}^{\perp}}\left\langle\hat{\Omega}^{\perp}\left|C^{\perp}\right| \hat{\Omega}^{\perp}\right\rangle}{\sqrt{\operatorname{det} C^{\perp}}},
$$

where the minimum is taken over all unit directions $\hat{\Omega}^{\perp}$ in the plane perpendicular to $\hat{\Omega}$. It turns out (details are given in Appendix $\mathrm{H}$ ) that $\lambda$ is a function of $\xi_{S}$ alone, namely $\lambda=\sqrt{1+\alpha \beta\left(\xi_{S}+1 / \xi_{S}-2\right)} / 2$, such that the entanglement entropy $S_{A}$ in Eq. (23) is a function of $\xi_{S}$ alone.

\subsection{Dynamics of entanglement}

Let us discuss the time dependence of $S_{A}$ after a quantum quench $\Gamma_{i} \rightarrow \Gamma_{f}$. How does the entanglement entropy scale in time after a quantum quench in the four different regimes of the dynamical phase diagram in Fig. 1?

We present two related views on the dynamics of entanglement. First, we discuss the intimate connection between the entanglement entropy and the variance of the collective spin state on the Bloch sphere. This point of view establishes the paradigm of spin squeezing. Second, we elaborate on the insight that the entanglement Hamiltonian is a harmonic oscillator whose angular frequency determines the entanglement spectrum and hence all Rényi entanglement measures.

The entanglement dynamics is tightly connected to the dynamics of the variance. We find that $\lim _{N \rightarrow \infty} \operatorname{det} N C^{\perp}=1 / 4^{2}$ (see Appendix H), i.e. there are two directions, call them $\hat{\Omega}_{1}$ and $\hat{\Omega}_{2}$, inside the $\hat{\Omega}_{1}^{\perp} \hat{\Omega}_{2}^{\perp}$ plane such that the uncertainty between $\mathbf{S} \cdot \hat{\Omega}_{1}$ and $\mathbf{S} \cdot \hat{\Omega}_{2}$ is minimized to leading order in $N$. Note that the eigenvalues of $C^{\perp}$ are $\operatorname{var}\left(\mathbf{S} \cdot \hat{\Omega}_{1}\right)$ and $\operatorname{var}\left(\mathbf{S} \cdot \hat{\Omega}_{2}\right)$. If both eigenvalues are exactly equal to the SQL, the variance is equally distributed in the $\hat{\Omega}_{1}^{\perp} \hat{\Omega}_{2}^{\perp}$ plane and the state is a non-entangled coherent spin state. However, if the variance of the magnetization along, say, $\hat{\Omega}_{1}$ is larger than the SQL, then the variance in the direction of $\hat{\Omega}_{2}$ must be below the SQL and the state is squeezed. The variance $\operatorname{var}\left(n_{+}\right)$of the magnetization in $z$-direction is a lower bound for the maximal eigenvalue of $C^{\perp}$. Hence, if $\operatorname{var}\left(n_{+}\right)$increases in time, the minimal eigenvalue of $C^{\perp}$ must decrease so that the state becomes squeezed and the individual spins become entangled. This is a qualitative reasoning why the von Neumann entanglement entropy increases as $\operatorname{var}\left(n_{+}\right)$increases.

The quantitative relation between the variance $\operatorname{var}\left(n_{+}\right)$and the entanglement entropy $S_{A}$ follows from Eq. (24). As the variance increases, $\xi$ in Eq. (23) approaches one from below and $S_{A}$ increases. In particular, if the variance $\operatorname{var}\left(n_{+}\right)$grows exponentially with time as in 

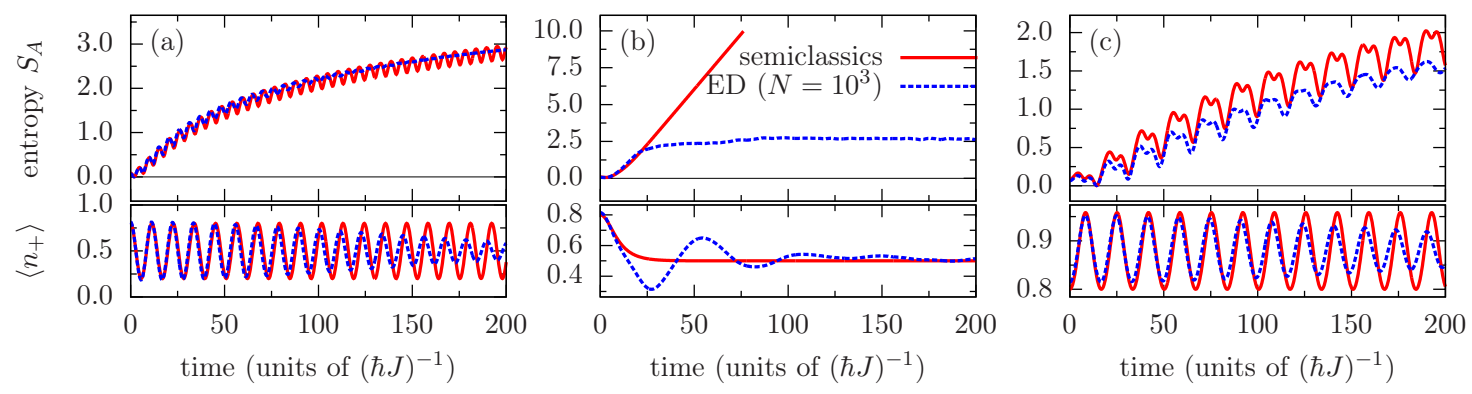

Figure 7: Dynamics of the von Neumann entanglement entropy $S_{A}$ for a symmetric bipartition (top), and spin expectation value $\left\langle n_{+}\right\rangle$(bottom) after a sudden quantum quench from $\Gamma_{i}=0.4$ to $\Gamma_{f}=0.8$ (a), $\Gamma_{f}=0.45$ (b), and $\Gamma_{f}=0.3$ (c) (cf. Fig. 1). The results are obtained by exact diagonalization with $N=10^{3}$ (dotted blue line) and by a leading order semiclassical expansion $\lim _{N \rightarrow \infty}\left\langle n_{+}\right\rangle$and $\lim _{N \rightarrow \infty} S_{A}$ (solid red line) according to Eqs. (4) and (23).
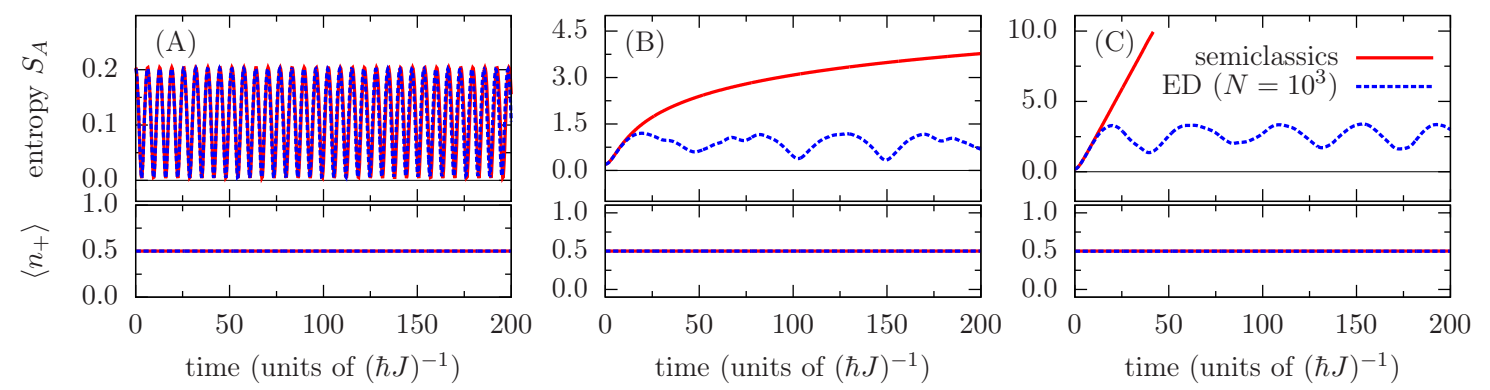

Figure 8: Dynamics of the von Neumann entanglement entropy $S_{A}$ for a symmetric bipartition (top), and spin expectation value $\left\langle n_{+}\right\rangle$(bottom) after a sudden quantum quench from $\Gamma_{i}=0.6$ to $\Gamma_{f}=0.8(\mathrm{~A}), \Gamma_{f}=0.5(\mathrm{~B})$, and $\Gamma_{f}=0.3$ (C) (cf. Fig. 1). The results are obtained by exact diagonalization with $N=10^{3}$ (dotted blue line) and by a leading order semiclassical expansion $\lim _{N \rightarrow \infty}\left\langle n_{+}\right\rangle$and $\lim _{N \rightarrow \infty} S_{A}$ (solid red line) according to Eqs. (4) and (23).

regime (I), the entanglement entropy increases linearly, cf. Fig. 7 (b) and Fig. 8 (C). In regime (II) where the variance oscillates and remains bounded over time, the entanglement entropy shows bounded oscillations, cf. Fig. 8 (A). Logarithmic entanglement growth can be observed in regimes (III), cf. Fig. 8 (B), and (IV), cf. Fig. 7 (a) and Fig. 7 (c), and is a consequence of the quadratic increase of the variance. Similar results for the von Neumann entropy and a similar semiclassical interpretation were obtained in [33] by the different, though related, approach of the two-boson method.

Another point of view is fascilitated by the fact that the entanglement Hamiltonian (19) is a harmonic oscillator with angular frequency $\omega$. How does $\omega(t)$ change as a function of time after the quench? Figures 9 and 10 display the time dependence of $\omega(t)$ for the quenches of Fig. 1. By inspection of (23) one infers that $\omega$ decreases as the von Neumann entropy $S_{A}=H_{2}\left(e^{-\omega}\right) /\left(1-e^{-\omega}\right)$ increases, and similarly for the other Rényi entropies in (22). This 

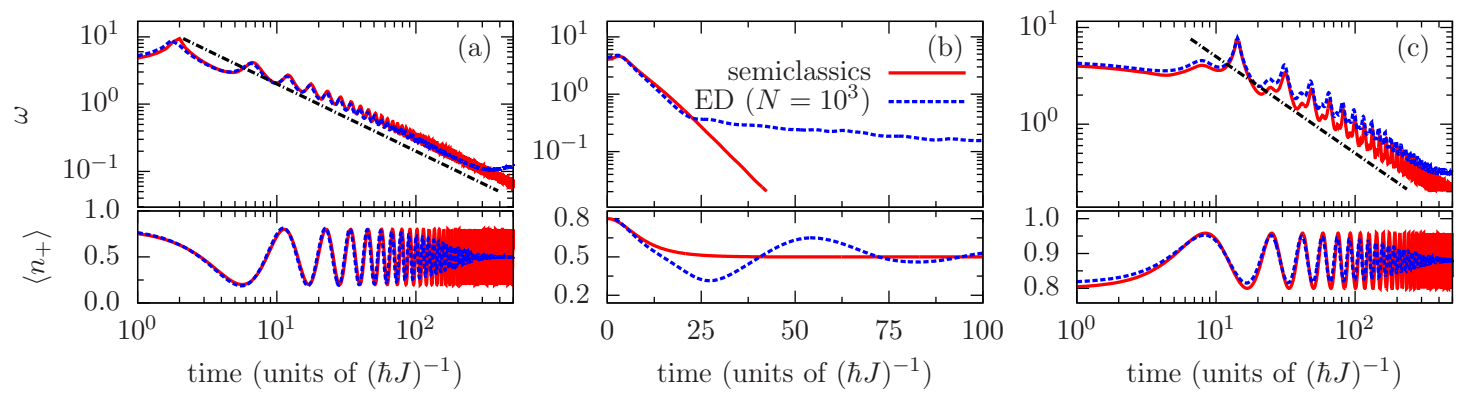

Figure 9: Dynamics of the angular frequency $\omega$ of the entanglement oscillator (19) for a symmetric bipartition (top), and spin expectation value $\left\langle n_{+}\right\rangle$(bottom) after a sudden quantum quench from $\Gamma_{i}=0.4$ to $\Gamma_{f}=0.8$ (a), $\Gamma_{f}=0.45$ (b), and $\Gamma_{f}=0.3$ (c) (cf. Fig. 1). The results are obtained by exact diagonalization with $N=10^{3}$ (dotted blue line) and by a leading order semiclassical expansion $N \rightarrow \infty$ according to Eq. (4), and Eqs. (21) and (24). Note that $\omega$ is plotted with double logarithmic scaling in (a) and (c), while the plot of $\omega$ in (b) is a semi-log plot. The black dashed line $\propto 1 / t$ in (a) and (c) is a guide to the eye.

has a natural interpretation in the language of thermodynamics. Instead of thinking of the angular frequency as a time dependent quantity, one can equivalently keep it at a fixed value, say $\omega_{0}=\omega(0)$, and put the time dependence into a scaling factor $\beta(t)$, that is $\omega(t)=\omega_{0} \beta(t)$. We refer to the scaling factor as the inverse entanglement temperature to emphasize the thermodynamic analogy. Small $\omega(t)$ corresponds to large entanglement temperature, so that many entanglement Hamiltonian eigenstates are similarly occupied, and the entanglement entropy of the reduced density is large. On the contrary, large $\omega(t)$ corresponds to small temperature implying that the occupation of high entanglement Hamiltonian eigenstates is suppressed, leading to small entanglement. In the limit $\omega(t) \rightarrow \infty$ the entanglement temperature is zero, such that only the ground state is occupied and the reduced density matrix is pure. More quantitatively, the late time asymptotics of $S_{A}(t) \rightarrow \infty$ and $\omega(t) \rightarrow 0$ are related by $S_{A}=-\log (\omega)+1+\mathcal{O}\left(\omega^{2}\right)$, cf. Eq. (23). Hence, linear growth $S_{A} \propto t$ of the entanglement entropy translates to exponential decrease $\omega \propto e^{-c t}$ of the angular frequency, equivalently, to exponential increase of the entanglement temperature, cf. Fig. 9 (b) and Fig. 10 (C). Logarithmic growth $S_{A} \propto \log (t)$ implies reciprocal decay $\omega \propto 1 / t$, equivalently, linear growth of the entanglement temperature, cf. Fig. 9 (a), (c) and Fig. 10 (B).

We close the discussion of the entanglement dynamics by noting a curious implication for local operations and classical communication (LOCC) protocols. A well known theorem in quantum information theory, see e.g. chapter 12 in [89], states that a pure state $\psi_{1}^{A B}$ on a bipartite Hilbert space $\mathcal{H}_{A} \otimes \mathcal{H}_{B}$ can be transformed into another pure state $\psi_{2}^{A B}$ by means of a LOCC protocol if, and only if, the sequence of eigenvalues of $\operatorname{Tr}_{B}\left|\psi_{1}^{A B}\right\rangle\left\langle\psi_{1}^{A B}\right|$ is majorized by the sequence of eigenvalues of $\operatorname{Tr}_{B}\left|\psi_{2}^{A B}\right\rangle\left\langle\psi_{2}^{A B}\right|$. Valid operations of LOCC protocols include measurements and manipulations of the quantum state by operators that act non-trivially only on one of the two factors $\mathcal{H}_{A}$ and $\mathcal{H}_{B}$ at a time, and classical processing of the measured information.

We apply this theorem to the collective spin states $\psi_{A B}\left(t_{1}\right)$ and $\psi_{A B}\left(t_{2}\right)$ at two different instants of time $t_{1}$ and $t_{2}$ after the quantum quench. In the large $N$ limit, and for times 

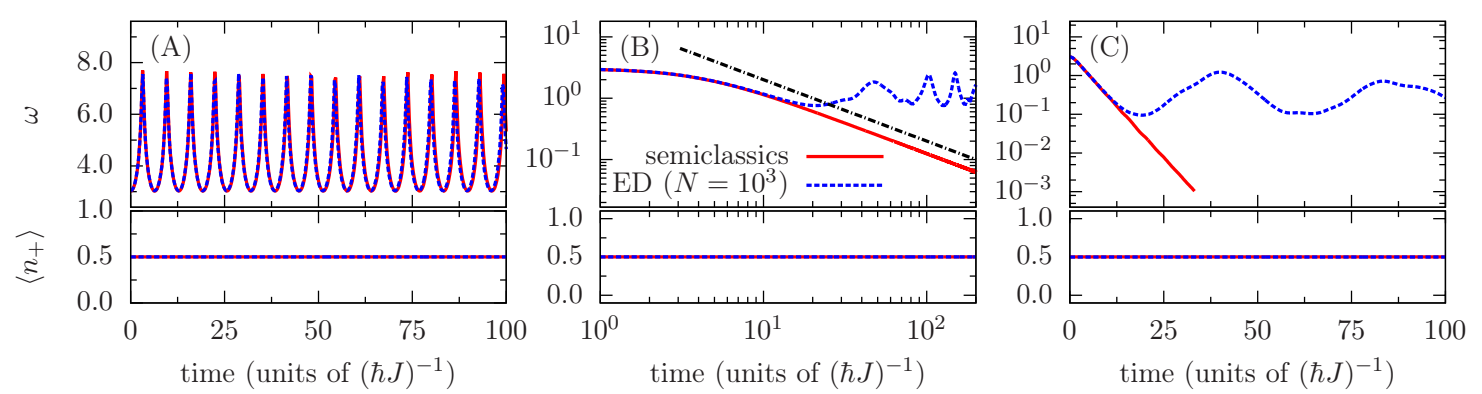

Figure 10: Similar to Fig. 9, showing the dynamics after a quantum quench from $\Gamma_{i}=0.6$ to $\Gamma_{f}=0.8(\mathrm{~A}), \Gamma_{f}=0.5(\mathrm{~B})$, and $\Gamma_{f}=0.3$ (C) (cf. Fig. 1). Note, the $\omega$ plot in (B) uses double logarithmic scaling, while the plot of $\omega$ in $(\mathrm{C})$ is a semi-log plot. The black dashed line $\propto 1 / t$ in (B) is a guide to the eye.

$t_{1}, t_{2}$ when the semiclassical analysis is valid, the non-increasing sequence of eigenvalues of the reduced states $\rho_{A}\left(t_{1}\right)$ and $\rho_{A}\left(t_{1}\right)$ is given by

$$
\begin{aligned}
& P_{1}=\left(1-\xi_{1}, \xi_{1}\left(1-\xi_{1}\right), \xi_{1}^{2}\left(1-\xi_{1}\right), \cdots\right), \text { and } \\
& P_{2}=\left(1-\xi_{2}, \xi_{2}\left(1-\xi_{2}\right), \xi_{2}^{2}\left(1-\xi_{2}\right), \cdots\right),
\end{aligned}
$$

respectively, where $\xi_{i}=e^{-\omega\left(t_{i}\right)}$, according to Eqs. (20) and (52). $P_{1}$ is majorized by $P_{2}$, i.e. $\left(1-\xi_{1}\right) \sum_{j=0}^{k} \xi_{1}^{j} \leq\left(1-\xi_{2}\right) \sum_{j=0}^{k} \xi_{2}^{j}$ for all integers $k \in \mathbb{N}_{0}$, if, and only if, $\xi_{1} \geq \xi_{2}$, equivalently $\lambda_{1} \geq \lambda_{2}$, where $\lambda_{i}=\left(1+\xi_{i}\right) /\left(1-\xi_{i}\right) / 2$ is the symplectic eigenvalue of the covariance of $\rho_{A}\left(t_{i}\right)$. We conclude that the unitary time evolution between two instants of time after the quantum quench can be realized by a LOCC protocol, if, and only if the symplectic eigenvalue $\lambda$ of the covariance of $\rho_{A}$ is non-increasing. On the contrary, when $\lambda$ increases between two instants of time, the time evolution cannot be realized by LOCC operations.

\section{Conclusion}

Interesting quantum many body systems, for which relevant quantities can be computed exactly or even approximately, are rare. In this paper, we have examined the fully connected transverse field Ising model as a simple, yet non-trivial, mean field model, which is amenable to a systematic mathematical expansion in inverse system size. This model is not only relevant experimentally, but can also be thought of as a prime model to benchmark the validity of mean field approximations out of equilibrium. Compared to equilibrium, it is less well understood when, i.e. up to which time scales, and how accurate mean field approximations are in non equilibrium situations. In the fully connected Ising model, a typical example of a mean field system, the approximation breaks down at surprisingly early times, scaling with the square root of the system size. This early breakdown happens away from unstable critical points and is explained on the basis of a dephasing effect leading to a linear in time spreading of the wave packet.

Based on the dynamics of the order parameter, i.e. the expected magnetization, and its 
variance we have discussed the dynamical phase diagram Fig. 1 for global quenches in the transverse magnetic field. We have seen how the behavior of the variance allows to discriminate different regions in the dynamical phase diagram, which cannot be distinguished by the order parameter alone.

We confirmed the quantitative connection between the variance, i.e. spin squeezing, and various entanglement measures. Remarkably, the entanglement Hamiltonian can be stated explicitly in the large system limit. The entanglement Hamiltonian is a time dependent harmonic oscillator, whose spectrum is known exactly and determines all Rényi entanglement entropies. The spectrum depends on the harmonic oscillator through the angular frequency, which in turn can be related to the determinant of the (co)variance of the Wigner transform of the wave function. Consequently, in the mean field transverse field Ising model, spin squeezing entails the full entanglement spectrum.

The key ingredient for a coherent picture of the mean field dynamics, as summarized by the dynamical phase diagram, is the interplay between the the expectation value and the variance of the order parameter. On the one hand, the variance neatly explains both, first, the (early) breakdown of the mean field approximation, as well as, second, the qualitative behavior of the entanglement entropy dynamics. On the other hand, the dynamics of the variance depends on the behavior of (the mean field limit of) the expectation value. This is demonstrated by the hierarchical structure of the ordinary differential equations governing the dynamics of the expectation value and its variance. Two situations, in which the influence of the expectation value on the variance becomes particularly clear, is, first, when the expectation value is close to a (stable or unstable) fixed point, and, second, when the expectation value follows a closed periodic orbit. The latter case leads to the subtle phenomenon of 'periodically enhanced squeezing and spreading' of the time evolving wave packet.

The energy landscape in an effective semiclassical phase space determines the center and variance of the time evolved wave packet and thereby the expectation value and variance of permutation invariant observables, such as the mean magnetization. When the wave packet is at an unstable fixed point, as for quenches from the paramagnetic (PM) phase to the ferromagnetic (FM) phase, or close to a homoclinic orbit connecting to the unstable fixed point, as for quenches on the critical line of the dynamical phase transition, the variance increases exponentially in time. For quenches from the PM phase to the FM phase the wave packet is centered at a stable fixed point, resulting in bounded oscillations of the variance, akin to the dynamics of a centered Gaussian wave function in an harmonic oscillator. This fixed point becomes degenerate for quenches from the PM phase to the quantum critical point separating the PM and FM phase. As a consequence of this degeneracy, the variance increases quadratically. The three distinct situations, (a) stable non-degenerate fixed point, (b) stable degenerate fixed point, and (c) unstable fixed point, have the exact same order parameter evolution, but can easily be distinguished by the variance.

For quenches starting in the FM phase away from the critical line of the dynamical phase transition, the behavior of the variance is not dominated by the fixed point structure of the energy landscape. Instead, we have elaborated how periodic orbits and deviations from it lead to squeezing of the wave packet in the presence of anharmonic terms in the Hamiltonian. This subtle dephasing mechanism leads to oscillations of the variance within an envelope of quadratically increasing and inversely quadratic decreasing bounds. We have referred to this observation as 'periodically enhanced squeezing and spreading'.

By comparing to exact diagonalization, we find perfect agreement for early times. However, even for large system sizes a dephasing mechanism leads to a deviation from the mean 
field approximation as time proceeds. The breakdown of mean field occurs at the Ehrenfest time, when the spread of the wave function, as measured by its variance, becomes comparable to the length scale on which anharmonic terms of the Hamiltonian cannot be neglected. As a consequence, qualitatively different dynamical behavior of the variance leads to different scaling of the timescale of validity with system size $N$. In particular, close to unstable fixed points, characterized by exponential increase of the variance, mean field results are only valid up to times scaling logarithmically in system size. For quenches in the regime of periodically enhanced squeezing and spreading, mean field breaks down on timescales of square root order in system size. Hence, also away from unstable critical points, mean field ceases to be valid after comparatively short times, even in large systems. The other extreme is a stable non-degenerate fixed points, at which the harmonic approximation of the Hamiltonian is particularly good, such that mean field remains valid up to times scaling linearly in system size.

Subsequently, we have shown that the entanglement Hamiltonian w.r.t. a bipartition of the spins into two disjoint sets is a harmonic oscillator. In analogy to thermodynamics, the angular frequency of this oscillator can be interpreted as the inverse entanglement temperature, which determines the entanglement spectrum, and thereby all Rényi entanglement entropies. Equivalently, the entanglement entropies have also been expressed as functions of a spin squeezing parameter, namely the fraction between the maximal and minimal spin variance in directions perpendicular to the mean magnetization. We thereby confirmed the quantitative relation between spin squeezing and the entanglement spectrum.

The observations about the dynamics of the variance, as contemplated in Fig. 1, translate to qualitative different behavior of the entanglement entropy as a function of time after the quench. More precisely, polynomial and exponential increases of the variance leads to logarithmic and linear growth of the entanglement entropy, respectively, while bounded oscillations imply bounded entanglement. In particular, the asymptotic growth of the entropy is logarithmic for quenches starting in the FM phase, i.e. in the regime of 'periodically enhanced squeezing and spreading'. For quenches from the PM phase to the FM phase, and from the PM phase to the PM phase the entropy shows linear growth and bounded oscillations, respectively, while the entropy grows logarithmically for quenches to the quantum critical point separating the FM and PM phase. Finally, quenches on the critical line of the dynamical phase transition are characterized by linear growth of entanglement entropy. To summarize, the different regimes of variance growth in the dynamical phase diagram translate to qualitatively different regimes of entanglement growth.

We expect that many of the above results hold more generally for quantum models in the semiclassical limit.

\section{Acknowledgements}

Discussions with Mariya Medvedyeva, Aditi Mitra, Giuseppe Mussardo, Salvatore Manmana, and Vincenzo Alba are greatly acknowledged.

Funding information This work was supported through SFB 1073 (project B03) of the Deutsche Forschungsgemeinschaft (DFG). 


\section{A Derivation of the effective Hamiltonian}

We want to solve the Schrödinger equation

$$
i \partial_{t}|\psi\rangle=\left(-N J S_{z}^{2} / 2-N \Gamma S_{x}\right)|\psi\rangle
$$

in the permutation invariant Dicke subspace. To this end, we expand the wave function in the Dicke states as $|\psi\rangle=\sum_{N_{+}} \psi\left(N_{+}\right)\left|N_{+}\right\rangle$and deduce the differential equation for the coefficients $\psi\left(N_{+}\right)=\left\langle N_{+} \mid \psi\right\rangle$. We obtain

$$
\begin{aligned}
i \partial_{t} \psi\left(N_{+}\right)= & -N \frac{J}{2}\left(\frac{N_{+}}{N}-\frac{1}{2}\right)^{2} \psi\left(N_{+}\right)-N \Gamma\left[\frac{1}{2} \frac{N_{+}+1}{N} \sqrt{\frac{\left(N-N_{+}-1\right)+1}{N_{+}+1}} \psi\left(N_{+}+1\right)\right. \\
& \left.+\frac{1}{2} \frac{N-N_{+}+1}{N} \sqrt{\frac{N_{+}}{N-N_{+}+1}} \psi\left(N_{+}-1\right)\right] \\
= & -N \frac{J}{2}\left(n_{+}-\frac{1}{2}\right)^{2} \psi\left(N_{+}\right)-N \Gamma\left[\frac{1}{2} \sqrt{\left(1-n_{+}\right)\left(n_{+}+1 / N\right)} \psi\left(N_{+}+1\right)\right. \\
& \left.+\frac{1}{2} \sqrt{n_{+}\left(1-n_{+}+1 / N\right)} \psi\left(N_{+}-1\right)\right] .
\end{aligned}
$$

The expression becomes more symmetric when expressed in terms of the magnetization per site $s=\left(N_{+} / N-1 / 2\right)$. Note that the magnetization per site can take $(N+1)$ possible equidistantly distributed values in the interval between $-1 / 2$ and $+1 / 2$. Hence, by a slight abuse of notation, we write $\psi(s)$ for $\psi\left(N_{+}=N(s+1 / 2)\right)$ and get

$$
\frac{i}{N} \partial_{t} \psi(s)=-\frac{J}{2} s^{2} \psi(s)-\Gamma \frac{1}{2}\left[\sqrt{\frac{1}{4}-s^{2}+\frac{1 / 2-s}{N}} \psi(s+1 / N)+\sqrt{\frac{1}{4}-s^{2}+\frac{1 / 2+s}{N}} \psi(s-1 / N)\right] .
$$

Introducing the shift operators ${ }^{1} \triangle_{ \pm}$by $\left(\triangle_{ \pm} \psi\right)(s)=\psi(s \pm 1 / N)$ (with the understanding that $\psi( \pm 1 / 2 \pm 1 / N)=0)$, yields

$$
\frac{i}{N} \partial_{t} \psi(s)=\left[-\frac{J}{2} s^{2}-\frac{\Gamma}{2} \sqrt{\frac{1}{4}-s^{2}}\left(\triangle_{+}+\triangle_{-}\right)+\epsilon_{1} \triangle_{+}+\epsilon_{2} \triangle_{-}\right] \psi(s),
$$

where $\epsilon_{1,2}(s)=\sqrt{\frac{1}{4}-s^{2}+\frac{1 / 2 \mp s}{N}}-\sqrt{\frac{1}{4}-s^{2}}$ are of order $1 / N$. No approximation has been made so far and the last expression describes the exact propagation in the Dicke subspace $\mathcal{D}_{N}$.

We may now approximate Eq. (28) in the limit of large $N$. The approximation is twofold. First, we assume that the $(N+1)$ dimensional vector $\psi(s)$ can be approximated by a smooth function of $s$. That is, we assume there is a smooth function $\phi$ defined on the continuous interval $[-1 / 2,1 / 2]$ such that $\psi(s)=\phi(s)+\mathcal{O}(1 / N)$ for all $s \in\{-1 / 2,-1 / 2+1 / N, \ldots, 1 / 2\}$. Under this assumption we may replace the shift operators $\triangle_{ \pm}$by the formal expression $e^{ \pm \partial_{s} / N}$.

\footnotetext{
${ }^{1}$ Let the shift operators $\triangle_{ \pm}$on $\mathbb{C}^{N+1}$ be defined by $\triangle_{+} \psi=\left(\psi_{1}, \ldots, \psi_{N}, 0\right)$ and $\triangle_{-} \psi=\left(0, \psi_{0}, \ldots, \psi_{N-1}\right)$. It is easy to see that $\triangle_{+}$and $\triangle_{-}$are adjoints of each other. More generally, the adjoint of $\operatorname{diag}(g) \triangle_{ \pm}$is $\operatorname{diag}\left(\triangle_{\mp} \bar{g}\right) \triangle_{\mp}$, where $g$ is the kernel of the diagonal operator $\operatorname{diag}(g)$. Hence, the operator $\left(g_{1} \triangle_{+}+g_{2} \triangle_{-}\right)$is Hermitian if $g_{1}=\triangle_{+} \bar{g}_{2}$. Now, for $g_{1,2}(s)=\sqrt{\frac{1}{4}-s^{2}+\frac{1 / 2 \mp s}{N}}$ one has $g_{1}(s)=\bar{g}_{2}(s+1 / N)$, which confirms that the operator on the right hand side of Eq. (27) is Hermitian.
} 
Second, we only consider the leading terms on the right hand side of Eq. (28), i.e. we drop the $\mathcal{O}(1 / N)$ terms. We thus obtain

$$
\frac{i}{N} \partial_{t} \phi(s)=\left[-\frac{J}{2} s^{2}-\Gamma \sqrt{\frac{1}{4}-s^{2}} \cos (p)\right] \phi(s),
$$

where $p=-i \partial_{s} / N$.

Equation (29) may be interpreted as an effective one dimensional Schrödinger equation for a single fictitious particle governed by the Hamiltonian $H(s, p)=-\frac{J}{2} s^{2}-\Gamma \sqrt{\frac{1}{4}-s^{2}} \cos (p)$. Note that the second term in the Hamiltonian is not Hermitian. This is an artifact of the approximation, in particular of the fact that we have neglect terms of order $1 / N$. The total magnetization per site plays the role of the particle's position and the inverse system size, $1 / N$, plays the role of an effective Planck constant. In the limit of large $N$, when the effective Planck constant is small, we will therefore apply semiclassical techniques to understand the dynamics imposed by Eq. (29).

\section{B Rate function expansion}

In this appendix we discuss the dynamics of the rate function in the neighborhood of its minimum and derive Eq. (7). More generally, we derive the differential equations for the Taylor coefficients of the rate function expansion around its minimum. The behavior of the Taylor coefficients determine the leading contribution of the order parameter and its variance, see Eqs. (4) and (5). The main result of this appendix is Eq. (7), which is a simple ordinary differential equation for the curvature of the rate function at the minimum. Remarkably, the curvature does not couple to higher derivatives of the rate function. We derive the more general result that the dynamics of $n$th derivative depends only on derivatives of smaller order than $n$.

The equations of motion for the complex rate function $f(x, t)$ is a nonlinear partial differential equation (PDE)

$$
\partial_{t} f(x, t)=i H\left(x, i \partial_{x} f(x, t)\right),
$$

compare Eq. (6). Let us assume that $\Re f(., t)$ has a unique global minimum $x_{\mathrm{cl}}(t)$ at all times $t$. Instead of solving the full PDE (30), we content ourselves with asking a more humble question: What constraints does the PDE (30) impose on the dynamics of $f$ in the neighborhood of $x_{\mathrm{cl}}$ ? To answer this question, we expand $f(x, t)=\sum_{n=0} f_{n}(t)\left[x-x_{\mathrm{cl}}(t)\right]^{n} / n$ ! in a Taylor series around $x_{\mathrm{cl}}$. Note that the Taylor coefficients

$$
f_{n}(t)=\left.\frac{\partial^{n} f(x, t)}{\partial x^{n}}\right|_{x_{\mathrm{cl}}(t)}
$$

are time dependent due to two reasons. First, because $f(x, t)$ is explicitly time dependent, and second, because $x_{\mathrm{cl}}(t)$ is in general time dependent. Therefore, the time derivative of $f_{n}$ gets two contributions,

$$
\partial_{t} f_{n}(t)=\left.\frac{\partial^{n} \partial_{t} f(x, t)}{\partial x^{n}}\right|_{x_{\mathrm{cl}}(t)}+\left.\frac{\partial^{n+1} f(x, t)}{\partial x^{n+1}}\right|_{x_{\mathrm{cl}}(t)} \dot{x}_{\mathrm{cl}}
$$


Applying Eq. (30) on the first term on the right hand side yields

$$
\partial_{t} f_{n}=\left.i \partial_{x}^{n} H\left(x, i \partial_{x} f\right)\right|_{x_{\mathrm{cl}}(t)}+f_{n+1} \dot{x}_{\mathrm{cl}}
$$

Note that, after evaluating the first term at $x=x_{\mathrm{cl}}(t)$, the right hand side is a function of $x_{\mathrm{cl}}(t)$ and $\left\{f_{n}\right\}_{n}$. Therefore, Eq. (31) is a system of coupled first order ordinary differential equations for $\left\{f_{n}\right\}$. Remarkably, as we shall prove below, the right hand side of Eq. (31) only seemingly depends on $f_{n+1}$. Hence, the coupling among the $f_{n}$ obeys a hierarchical structure in the sense that the equation of motion for $f_{n}$ only depend on coefficients $f_{m}$ of lower order $m<n$. As a consequence, the differential equations for the first, say, $n$ coefficients $f_{1}, \ldots f_{n}$ close and can be solved exactly.

We now prove the fact that the right hand side of (31) does not depend on $f_{m}$ with $m>n$ inductively. Starting with $n=1$, Eq. (31) reads $\dot{f}_{1}=i H^{(1,0)}\left(x_{\mathrm{cl}}, i f_{1}\right)-H^{(0,1)}\left(x_{\mathrm{cl}}, i f_{1}\right) f_{2}+$ $f_{2} \dot{x}_{\mathrm{cl}}(t)$. Here $H^{(n, m)}$ denotes the $n$th and $m$ th derivative of $H$ w.r.t. its first and second argument, respectively. By the definition of $x_{\mathrm{cl}}(t)$ being the minimum of $\Re f(., t)$, the real part of $f_{1}$ vanishes identically for all times. Writing $f_{1}(t)=-i p_{\mathrm{cl}}(t)$ for the imaginary part, gives $-i \dot{p}_{\mathrm{cl}}=i H^{(1,0)}\left(x_{\mathrm{cl}}, p_{\mathrm{cl}}\right)-H^{(0,1)}\left(x_{\mathrm{cl}}, p_{\mathrm{cl}}\right) f_{2}+f_{2} \dot{x}_{\mathrm{cl}}(t)$. The real and imaginary part of the last equation are Hamilton's equations of motion

$$
\begin{aligned}
& \dot{x}_{\mathrm{cl}}=H^{(0,1)}\left(x_{\mathrm{cl}}, p_{\mathrm{cl}}\right), \\
& \dot{p}_{\mathrm{cl}}=-H^{(1,0)}\left(x_{\mathrm{cl}}, p_{\mathrm{cl}}\right),
\end{aligned}
$$

as was already noted in Ref. [39]. Thus, the minimum of the rate function follows the classical trajectory and the rate function expansion is an expansion around the classical limit. Notice that the dependence on $f_{2}$ is canceled.

Proceeding inductively, it remains to show that the term $f_{n+1} \dot{x}_{\mathrm{cl}}$ on the right hand side of (31) is canceled for $n \geqslant 2$. In fact, the only term in the expression $\left.i \partial_{x}^{n} H\left(x, i \partial_{x} f\right)\right|_{x_{\mathrm{cl}}(t)}$ containing $f_{n+1}$ is $-H^{(0,1)}\left(z_{\mathrm{cl}}, p_{\mathrm{cl}}\right) f_{n+1}$. This term cancels the term $f_{n+1} \dot{x}_{\mathrm{cl}}(t)$ due to the equations of motion, which concludes the claim.

In particular, using $n=2$ in Eq. (31), gives the dynamics of $f_{2}$ in terms of the quadratic form

$$
\partial_{t} f_{2}=-i\left(-i, f_{2}\right) H^{\prime \prime}\left(-i, f_{2}\right),
$$

$H^{\prime \prime}$ being the Hessian matrix of the Hamiltonian evaluated at the classical trajectory $\left(x_{\mathrm{cl}}, p_{\mathrm{cl}}\right)$. This result is used to investigate the dynamics of the variance according to Eq. (5). It is also the starting point to prove the equivalence to the classical nearby orbit approximation, see Appendix C.

We close this appendix by stating the next to leading order extension of Eqs. (4a) and (5a),

$$
\begin{aligned}
\left\langle n_{+}\right\rangle & =n_{\mathrm{cl}}-\frac{g_{3}}{4 g_{2}^{2} N}+\mathcal{O}\left(N^{-2}\right), \\
\operatorname{var}\left(n_{+}\right) & =\frac{1}{2 g_{2} N}-\frac{g_{4}}{8 g_{2}^{3} N^{2}}+\frac{g_{3}^{2}}{4 g_{2}^{4} N^{2}}+\mathcal{O}\left(N^{-3}\right),
\end{aligned}
$$

where $g_{n}$ denotes the real part of $f_{n}$. These equations follow from a next to leading order saddle point approximation. 


\section{Nearby orbit vs. large deviation}

The purpose of this appendix is to show that the variance as computed within nearby orbit approximation, cf. Eq. (8), is identical to the result obtained by leading order rate function expansion, cf. Eq. (5). In the sequel, we write $H^{\prime \prime}=H^{\prime \prime}\left(z_{r}(t)\right)$ for the Hessian matrix of the classical Hamiltonian $H: \mathbb{R}^{2 n} \rightarrow \mathbb{R}$ evaluated at the reference orbit. The reference orbit is the solution of the equations of motion $\dot{z}_{r}=J H^{\prime}\left(z_{r}\right)$ with initial condition $z_{r}(0)=z_{0}$. For the sake of simplicity, we restrict to the case $n=1$. All arguments apply for $n>1$ as well, but the calculation becomes more lengthy.

More precisely, let $C_{\mathrm{NO}}(t)=S(t) C_{\mathrm{NO}}(0) S(t)^{T}$ be the nearby orbit covariance matrix, where $S(t)$ is the fundamental matrix of the differential equation $\dot{S}(t)=J H^{\prime \prime} S(t)$ with $S(0)=$ id. And, let

$$
C_{\mathrm{LD}}(t)=\frac{1}{2 N}\left(\begin{array}{cc}
\left(\Re f_{2}\right)^{-1} & -\Im f_{2} / \Re f_{2} \\
-\Im f_{2} / \Re f_{2} & 1 / \Re\left(f_{2}^{-1}\right)
\end{array}\right)
$$

be the covariance matrix as obtained within the large deviation formalism (see Appendix B), where $\partial_{t} f_{2}=-i\left(-i, f_{2}\right) H^{\prime \prime}\left(-i, f_{2}\right)$, see Eq. (7). We prove the following claim: If the two covariance matrices initially coincide, that is $C_{\mathrm{NO}}(0)=C_{\mathrm{LD}}(0)$, then they agree for all later times as well, i.e. $C_{\mathrm{NO}}(t)=C_{\mathrm{LD}}(t)$ for all $t$.

We look at the difference $D(t)=C_{L D}(t)-C_{N O}(t)$ between the covariance matrix in large deviation and nearby orbit approximation. By assumption, one has $D(0)=0$. It remains to show that $D(t)=0$ for all $t>0$. The derivative of $C_{\mathrm{NO}}(t)$ is

$$
\frac{d}{d t} C_{\mathrm{NO}}(t)=J H^{\prime \prime} C_{\mathrm{NO}}(t)-C_{\mathrm{NO}}(t) H^{\prime \prime} J
$$

The time derivative of each matrix element of $C_{L D}$ follows from Eq. (7):

$$
\begin{aligned}
\frac{d}{d t}\left(\Re f_{2}\right)^{-1} & =-\left(\Re f_{2}\right)^{-2} \Re \dot{f}_{2} \\
& =-\left(\Re f_{2}\right)^{-2} \Re\left[-i\left(-i, f_{2}\right) H^{\prime \prime}\left(-i, f_{2}\right)^{T}\right] \\
& =-2\left(\Re f_{2}\right)^{-2}\left[\Re\left(-i, f_{2}\right) H^{\prime \prime} \Im\left(-i, f_{2}\right)^{T}\right] \\
& =2\left(\begin{array}{ll}
0, & 1
\end{array}\right) H^{\prime \prime}\left(\begin{array}{c}
\left(\Re f_{2}\right)^{-1} \\
-\Im f_{2} / \Re f_{2}
\end{array}\right),
\end{aligned}
$$

and similarly, one obtains

$$
\begin{aligned}
\frac{d}{d t} \Re\left(f_{2}^{-1}\right)= & -2\left(\begin{array}{ll}
1, & 0
\end{array}\right) H^{\prime \prime}\left(\begin{array}{c}
-\Im f_{2} / \Re f_{2} \\
\Re\left(f_{2}^{-1}\right)
\end{array}\right), \\
-\frac{d}{d t} \frac{\Im f_{2}}{\Re f_{2}}= & -\left(\begin{array}{ll}
1, & 0
\end{array}\right) H^{\prime \prime}\left(\begin{array}{c}
\left(\Re f_{2}\right)^{-1} \\
-\Im f_{2} / \Re f_{2}
\end{array}\right) \\
& +\left(\begin{array}{ll}
0, & 1
\end{array}\right) H^{\prime \prime}\left(\begin{array}{c}
-\Im f_{2} / \Re f_{2} \\
\Re\left(f_{2}^{-1}\right)
\end{array}\right) .
\end{aligned}
$$

The last three equations can be written in a unified matrix form as

$$
\frac{d}{d t} C_{\mathrm{LD}}(t)=J H^{\prime \prime} C_{\mathrm{LD}}(t)-C_{\mathrm{LD}}(t) H^{\prime \prime} J
$$


Subtracting Eqs. (33) and (34), we see that the difference $D(t)=C_{L D}(t)-C_{N O}(t)$ fulfills the first order differential equation

$$
\frac{d}{d t} D(t)=J H^{\prime \prime} D(t)-D(t) H^{\prime \prime} J
$$

with initial condition $D(0)=0$, which is uniquely solved by $D(t)=S(t) D(0) S(t)^{T}=0$.

\section{Nearby orbit approximation for periodic orbits}

We have investigated the dynamics of the order parameter and its variance in mean field models after a quantum quench in Sec. 4. In regime (IV), cf. Fig. 1, when the order parameter oscillates periodically, the short time dynamics of the variance shows quasi-periodic breathing. The envelope of these quasi-periodic oscillations shows two distinct features. First, the local maxima of the variance increase quadratically with time. Second, the local minima of the variance decrease inversely quadratic with time. We refer to the latter property as periodically enhanced squeezing. In this appendix we explain that the two features are the consequence of a common cause. In particular, we demonstrate how the observations follow from shearing effects of the quasi-probability distribution as a consequence of non-quadratic interaction terms in the Hamiltonian. As we will see, the non-quadratic terms are a sine qua non ingredient and the precise form of these terms is not important. This not only illustrates the crucial role of the non-quadratic terms, but also indicates the universality of our results independent of the details of the Hamiltonian. The periodicity of the order parameter is crucial for our argument as it enables the application of Floquet's theorem, which plays a key role.

The periodic squeezing is already captured by the leading order of a rate function expansion. As shown in Appendix $\mathrm{C}$ the dynamics of the variance to leading order is identical to the Gaussian covariance as obtained in nearby orbit approximation. We may thus use the phase space picture facilitated by the nearby orbit approximation to gain an intuitive understanding.

We consider the time-independent Hamiltonian $H(z)$ and its associated Hamiltonian flow $T_{t}(z)$ on the $2 n$ dimensional phase space, whose coordinates are denoted by $z=(x, p)$. More specifically, $T_{t}\left(z_{0}\right)$ is the solution of Hamilton's equations of motion, $\dot{z}=J H^{\prime}(z)$, that passes through $z_{0}$ at time $t=0$. In the sequel, the prime denotes differentiation w.r.t. phase space coordinates $z$ and $J$ is the standard symplectic form. Let $z_{r}(t)=T_{t}\left(z_{0}\right)$ be a $T$-periodic reference orbit. When approximated to first order around $z_{r}$, Hamilton's equations impose the differential equation

$$
\dot{\delta} z=\left.J H^{\prime \prime}\right|_{z_{r}(t)} \delta z
$$

on the deviation $\delta z=\left(z-z_{r}\right)$ from the reference orbit. Equation (35) is a first order nonautonomous differential equation with $T$-periodic coefficients. Consequently, the Floquet theorem [90] can be applied. It states that any fundamental matrix $S(t)$ of Eq. (35) decomposes into the product $S(t)=P(t) e^{t B}$. Here, $P(t)$ is a $T$-periodic complex non-singular $2 n$ square matrix and $B$ is a constant complex $2 n$ square matrix. We refer to $e^{T B}$ as the monodromy matrix and call its eigenvalues the Floquet multipliers. The Floquet multipliers are unique. From now on, we focus on the fundamental system with initial condition $S(0)=P(0)=$ id. 
Formally, this can be written as $S(t)=\mathcal{T} \exp \left(\left.\int_{0}^{t} J H^{\prime \prime}\right|_{z_{r}\left(t^{\prime}\right)} d t^{\prime}\right)$, where $\mathcal{T}$ denotes time ordering. Note that $S(t)$ is symplectic because it is the linear approximation to the Hamiltonian flow, $S(t)=\left.T_{t}^{\prime}(z)\right|_{z=z_{0}}$. Consequently, also the monodromy matrix is symplectic.

Importantly, because Eq. (35) is obtained by linearizing the equations of motion around $z_{r}(t)$, the time derivative $\dot{z}_{r}(t)$ is a solution of (35). Since $z_{r}$ is $T$-periodic, so is $\dot{z}_{r}$. Therefore, at least one of the Floquet multipliers is equal to unity. The corresponding eigenspace is spanned by $\dot{z}_{r}(0)$ and is tangent to the energy hypersurface at $z_{0}$ in the direction of the reference orbit. This follows readily. As $\dot{z}_{r}$ solves (35), it can be written as $\dot{z}_{r}(t)=S(t) \dot{z}_{r}(0)=$ $P(t) e^{t B} \dot{z}_{r}(0)$. The periodicity, $\dot{z}_{r}(t+T)=\dot{z}_{r}(t)$, then yields $e^{T B} \dot{z}_{r}(0)=\dot{z}_{r}(0)$. Moreover, as $e^{T B}$ is symplectic, the roots of its characteristic polynomial come in inverse pairs. Hence, the characteristic polynomial has at least one second root equals unity (we cannot conclude that there is a second Floquet multiplier equals unity because $e^{T B}$ might not be diagonalizable, see below).

From now on, let us consider the case $n=1$, when the monodromy matrix is two by two and its characteristic polynomial has a two-fold degenerate root equals one. In an appropriate basis this matrix takes the form of a shear matrix

$$
e^{T B}=\left(\begin{array}{cc}
1 & \alpha \\
0 & 1
\end{array}\right)
$$

with shear factor $\alpha$. The fundamental matrix is only periodic for $\alpha=0$. This case is for example realized by harmonic Hamiltonians (see below). In general, one has to allow for $\alpha \neq 0$, since the monodromy matrix might not be diagonalizable. An orthonormal basis in which the monodromy matrix takes the form (36) is given by the unit vector tangent to the energy hypersurface at $z_{0}$ and the unit vector in the direction of $H^{\prime}\left(z_{0}\right)$. We conclude,

$$
S(t)=P(t) M(t),
$$

with shear matrix

$$
M(t)=\left(\begin{array}{cc}
1 & \alpha t / T \\
0 & 1
\end{array}\right)
$$

We illustrate the consequences of this finding for localized phase space probability distributions. Consider a Gaussian probability distribution $\mu_{0}(z)$ initially localized at $z_{0}$ with covariance $C(0)$. The time evolved distribution at a later time $t$ is given by $\mu_{t}(z)=\mu_{0}\left(T_{-t} z\right)$. For early times and narrow initial covariance, the nearby orbit approximation predicts that $\mu_{t}$ is close to a Gaussian distribution centered at $T_{t}\left(z_{0}\right)$ with covariance $C(t)=S(t) C(0) S(t)^{T}$ $[53,54]$. It follows from Eq. (37) that the time evolved covariance is obtained by consecutively shearing and periodically modulating the initial covariance. The shear factor $\alpha t / T$ is proportional to time. The variance in the direction of the unit vector $v$ is then determined by the quadratic form $C_{v}(t)=\langle v|C(t)| v\rangle$. $C_{v}(t)$ oscillates within the range set by the eigenvalues of $C(t)$. Using the form of $S(t)$ as contemplated in Eq. (37), one reads off that the oscillatory behavior of $C_{v}(t)$ comes from the periodic modulation by $P(t)$. The envelope of these oscillations is determined by the shear matrix $M(t)$. For the sake of simplicity, let us assume $C(0)=\operatorname{diag}\left(\lambda_{1}, \lambda_{2}\right)$ is diagonal in the basis in which Eq. (37b) holds. Then the eigenvalues of $M(t) C(0) M(t)^{T}$ are given by

$$
\lambda_{1,2}(t)=\frac{1}{2}\left[\left(\alpha \frac{t}{T}\right)^{2} \lambda_{1}+\operatorname{tr}\right]\left[1 \pm \sqrt{1-\frac{4 \operatorname{det}}{\left(\alpha \frac{t}{T}\right)^{2} \lambda_{1}+\operatorname{tr}}}\right],
$$


where $\operatorname{tr}=\lambda_{1}+\lambda_{2}$ and det $=\lambda_{1} \lambda_{2}$. For late times, $t \gg T, \lambda_{1}(t)=(\alpha t / T)^{2} \lambda_{1}+\operatorname{tr}+\mathcal{O}\left(t^{-2}\right)$ increases quadratically with time whereas $\lambda_{2}(t)=\operatorname{det} /\left[(\alpha t / T)^{2} \lambda_{1}+\operatorname{tr}\right]+\mathcal{O}\left(t^{-6}\right)$ decreases inversely quadratic with time. This explains the quadratic increase and the periodically enhances squeezing of the variance.

\section{D.1 Interpretation of $\alpha$}

In the following we derive an explicit expression for the shearing factor $\alpha$ given in Eqs. (38) and (39) below. We will show that a necessary and sufficient condition to observe shearing is that the period of the reference orbit differs from the period of nearby orbits.

The $T$-periodic reference orbit $z_{r}(t)=T_{t}\left(z_{0}\right)$ traverses a level set of the Hamiltonian at energy $E_{0}=H\left(z_{0}\right)$. Now, consider an initial deviation from the reference orbit in the direction perpendicular to the energy hypersurface, that is $\delta(0)=\epsilon H^{\prime}\left(z_{0}\right) /\left\|H^{\prime}\left(z_{0}\right)\right\|^{2}$ for some infinitesimal $\epsilon$. The normalization is chosen such that the energy of this nearby orbit differs from $E_{0}$ by $\epsilon, H\left(z_{0}+\delta(0)\right)=E_{0}+\epsilon+\mathcal{O}\left(\epsilon^{2}\right)$. For small enough $\epsilon$ the orbit starting at $z_{0}+\delta(0)$ is also closed, but in general the period is different from the period of the reference orbit. To leading order in $\epsilon$ the period is given by $T\left(E_{0}\right)+\epsilon T^{\prime}\left(E_{0}\right)$, where $T(E)$ denotes the period of an orbit at energy $E$ close to the reference orbit. An explicit expression of $T^{\prime}\left(E_{0}\right)$ is given below in Eq. (39). After time $T$ the initial position $z_{0}+\delta(0)$ has evolved to $T_{T}\left(z_{0}+\delta(0)\right)=z_{0}+S(T) \delta(0)+\mathcal{O}\left(\epsilon^{2}\right)$ under the Hamiltonian flow. By the definition of $\delta(0)$ and Eq. (37) one has $S(T) \delta(0)=\epsilon \alpha \dot{z}_{r}(0) /\left\|H^{\prime}\left(z_{0}\right)\right\|^{2}+\delta(0)$. As the difference $d z=$ $T_{T}\left(z_{0}+\delta(0)\right)-\left(z_{0}+\delta(0)\right)=\epsilon \alpha \dot{z}_{r}(0) /\left\|H^{\prime}\left(z_{0}\right)\right\|^{2}+\mathcal{O}\left(\epsilon^{2}\right)$ is infinitesimal but does not vanish unless $\alpha=0$, the period of the nearby orbit must be different from $T$ if $\alpha \neq 0$. More precisely, comparing to Hamilton's equations, $d z=d t J H^{\prime}$, one sees that the period of the nearby orbit differs by $d t=\epsilon \alpha /\left\|H^{\prime}\left(z_{0}\right)\right\|^{2}+\mathcal{O}\left(\epsilon^{2}\right)$ from the period of the reference orbit. Together with $d t=\epsilon T^{\prime}\left(E_{0}\right)+\mathcal{O}\left(\epsilon^{2}\right)$ one obtains

$$
\alpha=\left\|H^{\prime}\left(z_{0}\right)\right\|^{2} T^{\prime}\left(E_{0}\right) .
$$

The shearing factor is proportional to the change of the period of nearby orbits at different energies. The derivative is explicitly given by the integral

$$
T^{\prime}\left(E_{0}\right)=-\int_{0}^{T} \frac{H^{\prime}\left(J H^{\prime \prime} J+H^{\prime \prime}\right) H^{\prime}}{\left\|H^{\prime}\right\|^{4}} d t
$$

where $H^{\prime}$ and $H^{\prime \prime}$ are evaluated at $z_{r}(t)$ and the integration is over the full period of the reference orbit. An application of the two dimensional Stokes theorem yields $T^{\prime}\left(E_{0}\right)=$ $\iint_{\Sigma\left(E_{0}\right)} \operatorname{div}\left[\frac{\left(J H^{\prime \prime} J+H^{\prime \prime}\right) H^{\prime}}{\left\|H^{\prime}\right\|^{4}}\right] d z$, where the integral is over the surface $\Sigma\left(E_{0}\right)$ enclosed by the periodic orbit $z_{r}(t)$.

To derive Eq. (39), first note that the period of $z_{r}(t)$ is the integral $T\left(E_{0}\right)=\int \delta(H(z)-$ $\left.E_{0}\right) d^{2} z$. This follows from the equations of motion and $d^{2} z=d E d \sigma_{E}(z) /\left\|H^{\prime}(z)\right\|$, where $d \sigma_{E}(z)$ denotes the surface measure on the energy hypersurface $\{z: H(z)=E\}$ :

$$
\begin{aligned}
T\left(E_{0}\right) & =\int \delta\left(H(z)-E_{0}\right) d^{2} z=\int \frac{d \sigma_{E_{0}}}{\left\|H^{\prime}(z)\right\|} \\
& =\int \frac{\left\|\dot{z}_{r}(t)\right\|}{\left\|H^{\prime}(z)\right\|} d t=\int d t
\end{aligned}
$$


(assuming, for the sake of simplicity, that the level set $\left\{z: H(z)=E_{0}\right\}$ consists of a single connected component given by the reference orbit). Straightforward computation then yields

$$
\begin{aligned}
T\left(E_{0}+\epsilon\right)= & T\left(E_{0}\right)-\epsilon \int \delta^{\prime}\left(E-E_{0}\right) \frac{d \sigma_{E}(z)}{\left\|H^{\prime}(z)\right\|} d E+\mathcal{O}\left(\epsilon^{2}\right) \\
= & T\left(E_{0}\right)+\epsilon \int\left(\left.\frac{\partial}{\partial \epsilon}\right|_{\epsilon=0} \frac{d \sigma_{E_{0}+\epsilon}(z)}{\left\|H^{\prime}(z)\right\|}\right)+\mathcal{O}\left(\epsilon^{2}\right) \\
= & T\left(E_{0}\right)+\epsilon \int_{0}^{T}\left(\left.\frac{\partial}{\partial \epsilon}\right|_{\epsilon=0} \frac{\left\|\dot{z}_{r, \epsilon}\right\|}{\left\|H^{\prime}\left(z_{r, \epsilon}\right)\right\|}\right) d t \\
& +\mathcal{O}\left(\epsilon^{2}\right),
\end{aligned}
$$

where $z_{r, \epsilon}(t)=z_{r}(t)+\epsilon H^{\prime}\left(z_{r}(t)\right) /\left\|H^{\prime}\left(z_{r}(t)\right)\right\|^{2}$ is a parametrization of the hypersurface $\{z$ : $\left.H(z)=E_{0}+\epsilon\right\}$. Using the equations of motion $\dot{z}_{r}=J H^{\prime}$, in particular, $\left\|\dot{z}_{r}\right\|=\left\|H^{\prime}\right\|$ and $\dot{z}_{r} \cdot H^{\prime}=0$, eventually gives Eq. (39).

\section{D.2 Example}

In the remainder of this appendix we discuss a family of planar Hamiltonians that are amenable to explicit calculations. The example illustrates that non-harmonic terms in the Hamiltonian are necessary in order to have $\alpha \neq 0$. We investigate the class of classical Hamiltonians that depend on the phase space coordinates $z=(x, p) \in \mathbb{R}^{2}$ only through its Euclidean distance $\|z\|=\sqrt{x^{2}+p^{2}}$. In other words,

$$
H(z)=h\left(\|z\|^{2} / 2\right)
$$

for some function $h: \mathbb{R} \rightarrow \mathbb{R}$. The distance $\|z\|^{2}$ is an integral of motion of Hamilton's equations $\dot{z}=J H^{\prime}(z)=h^{\prime}\left(\|z\|^{2} / 2\right) J z$. The solution that passes through $z_{0}$ at $t=0$ is thus

$$
T_{t}\left(z_{0}\right)=P\left(t, z_{0}\right) z_{0},
$$

where $P\left(t, z_{0}\right)=e^{t \omega\left(z_{0}\right) J}$ and $\omega\left(z_{0}\right):=h^{\prime}\left(\left\|z_{0}\right\|^{2} / 2\right)$. Note that $\left\{P\left(t, z_{0}\right)\right\}_{t}$ is a $T=2 \pi / \omega\left(z_{0}\right)$ periodic one-parameter family in the group of orthogonal matrices. The integral curves are thus circles in phase space, which are traversed at a constant angular velocity $\omega\left(z_{0}\right)$. Generically, $\omega$ depends on the initial position. The angular velocity is only independent of the initial condition if $h^{\prime}$ is constant, i.e. when the Hamiltonian is quadratic. A non-constant angular velocity leads to shearing effects of probability distributions and shall be explained in the following.

Taking the derivative of Eq. (40) w.r.t. $z_{0}$ yields

$$
S(t)=P\left(t, z_{0}\right) \cdot\left[1+t \Omega\left(z_{0}\right) J z_{0} \otimes z_{0}\right]
$$

$(a \otimes b)_{i j}=a_{i} b_{j}$ being the dyadic product and $\Omega\left(z_{0}\right)=h^{\prime \prime}\left(\left\|z_{0}\right\|^{2} / 2\right)$. In the harmonic case, when $h^{\prime \prime}=0$, the last term vanishes and $S(t)=P\left(t, z_{0}\right)$ is periodic in time. Moreover, for $\Omega=0, S(t)$ is an orthogonal matrix and $C(t)=S(t) C(0) S(t)^{T}$ is $2 \pi / \omega$-periodic. As a consequence, the variance along any fixed direction (in particular, along the $x$ and $p$ direction) shows periodic breathing.

We will now focus on the less trivial non-harmonic situation and assume $\Omega\left(z_{0}\right) \neq 0$. Without loss of generality and for the sake of clarity, we set $z_{0}=\left(x_{0}, 0\right)$ to obtain

$$
S(t)=P\left(t, z_{0}\right)\left(\begin{array}{cc}
1 & 0 \\
-x_{0}^{2} \Omega t & 1
\end{array}\right) .
$$


This is of the same general form as predicted by Floquet's theorem in Eq. (37). One can read off the shearing factor $\alpha=-x_{0}^{2} T \Omega$, which agrees with Eqs. (38) and (39). The time evolved covariance $C(t)=S(t) C(0) S(t)^{T}$ is hence obtained by consecutively shearing and rotating the initial covariance. Whereas the rotation $P\left(t, z_{0}\right)$ is periodic in time, the shearing factor $-x_{0}^{2} \Omega t$ is proportional to time. Interestingly, the shearing factor depends only through the curvature $\Omega$ on the Hamiltonian but is independent of other details.

\section{E Nearby orbit approximation at fixed points}

In the previous appendix D we have discussed the dynamics of the covariance matrix within nearby orbit approximation in the case when the reference orbit is periodic. A limiting case occurs when the period of the reference orbit vanishes, i.e. when the reference orbit is a single critical point $z_{0}$ of the Hamiltonian, that is $H^{\prime}\left(z_{0}\right)=0$. Then, $z_{0}$ is a fixed point of the Hamiltonian flow and the solution of

$$
\dot{S}=J K S
$$

is $S(t)=\exp (J K t)$, where $K=H^{\prime \prime}\left(z_{0}\right)$. Note that $S$ obeys an autonomous differential equation and no time ordering is needed for the exponential. Let us restrict to $n=1$ when $K$ is a symmetric two by two matrix. The real eigenvalues $\lambda_{1}$ and $\lambda_{2}$ of $K$ determine the eigenvalues of $J K$ and therefore the dynamics of $S(t)$. This is only true for $n=1$ and is a manifestation of the fact that every two by two orthogonal matrix is also symplectic. To see this, let $O$ be the orthogonal matrix that diagonalizes $K$, i.e. $O K O^{T}=\operatorname{diag}\left(\lambda_{1}, \lambda_{2}\right)$. Then $O J K O^{T}=J \operatorname{diag}\left(\lambda_{1}, \lambda_{2}\right)$, where we have used that $O$ is also symplectic, i.e. $O J O^{T}=J$ (this is no longer true in general for $n>1$ ). This shows that the eigenvalues of $O J K O^{T}$ and thus the eigenvalues of $J K$ only depend on the eigenvalues of $K$. Note that for $n>1$ the eigenvalues of $J K$ do not solely depend on the eigenvalues of $K$ but also on the direction of the corresponding eigenvectors. For instance, let $n=2$ and assume $K$ has two positive and two negative eigenvalues. If the two negative eigendirections lie in the $\left(x_{1}, p_{1}\right)$ plane, then the classical trajectories close to the fixed point are related to ellipses and all eigenvalues of $J K$ are purely imaginary. However, if the two eigendirections of the negative eigenvalues lie in the $\left(x_{1}, x_{2}\right)$ plane, then the classical orbits close to the fixed point resemble hyperbolas and all eigenvalues of $J K$ are real. An orthogonal transformation rotating $K$ of the latter case into $K$ of the former case cannot be symplectic.

From now on, we assume $n=1$ and discuss the following cases: (i) $\lambda_{1}$ and $\lambda_{2}$ have the same sign, (ii) $\lambda_{1}$ and $\lambda_{2}$ have different signs, (iii) exactly one of $\lambda_{1}$ and $\lambda_{2}$ vanishes.

In the first case, $z_{0}$ is a maximum (negative eigenvalues) or a minimum (positive eigenvalues) of $H$ and the fixed point is elliptic, that is the eigenvalues of $J K$, being $\pm i \sqrt{\left|\lambda_{1} \lambda_{2}\right|}= \pm i \omega$, are purely imaginary. $S(t)$ is $T=2 \pi / \omega$ periodic and is explicitly given by

$$
O S(t) O^{T}=\left(\begin{array}{cc}
\cos \omega t & \sqrt{\lambda_{2} / \lambda_{1}} \sin \omega t \\
-\sqrt{\lambda_{1} / \lambda_{2}} \sin \omega t & \cos \omega t
\end{array}\right) .
$$

As a consequence, the covariance matrix $C(t)=S(t) C(0) S(t)^{T}$ oscillates periodically in time.

In the second case, $z_{0}$ is a saddle point of $H$ and the fixed point is hyperbolic, that is the eigenvalues of $J K$, being $\pm \sqrt{\left|\lambda_{1} \lambda_{2}\right|}= \pm \omega$, are real with opposite signs. Analogous to 
Eq. (42), one has

$$
O S(t) O^{T}=\left(\begin{array}{cc}
\cosh \omega t & \sqrt{\left|\lambda_{2} / \lambda_{1}\right|} \sinh \omega t \\
\sqrt{\left|\lambda_{1} / \lambda_{2}\right|} \sinh \omega t & \cosh \omega t
\end{array}\right) .
$$

The stable and unstable manifold of the hyperbolic fixed point are Hamiltonian level sets and cross at the fixed point. Let $\left|v_{-}\right\rangle$and $\left|v_{+}\right\rangle$be the unstable and stable manifold, respectively, then

$$
S(t)=e^{-\omega t}\left|v_{-}\right\rangle\left\langle w_{-}\left|+e^{\omega t}\right| v_{+}\right\rangle\left\langle w_{+}\right|,
$$

where $\left\langle w_{i} \mid v_{j}\right\rangle=\delta_{i, j}$, and $\left|v_{ \pm}\right\rangle$and $\left\langle w_{ \pm}\right|$are right and left eigenvectors of $S(t)$, respectively. In general, we have to distinguish right and left eigenvectors, because $S(t)$ is not symmetric (unless $\left|\lambda_{1}\right|=\left|\lambda_{2}\right|$ ). For late times, the covariance matrix $C(t)=S(t) C(0) S(t)^{T}$ may be approximated by $C(t)=C_{w_{+}} e^{2 \omega t}\left|v_{+}\right\rangle\left\langle v_{+}\right|+\mathcal{O}\left(e^{\omega t}\right)$, assuming that $C_{w_{+}}=\left\langle w_{+}|C(0)| w_{+}\right\rangle$does not vanish. In other words, for late times one eigendirection of $C(t)$ approaches the direction of the unstable manifold and the corresponding eigenvalue increases exponentially in time. As the phase space volume is preserved under the Hamiltonian flow $(\operatorname{det} S=1)$, there is also a direction in which the covariance decreases exponentially for large times. Due to the symmetry of $C(t)$, this direction is orthogonal to the direction of exponential spreading and becomes orthogonal to $\left|v_{+}\right\rangle$, i.e. parallel to $\left|w_{-}\right\rangle$, for late times. Note that in general, unless $\left|\lambda_{1}\right|=\left|\lambda_{2}\right|,\left|w_{-}\right\rangle$is not the direction of the stable manifold.

In the third case, the fixed point is degenerate and one has

$$
O S(t) O^{T}=\left(\begin{array}{cc}
1 & \lambda_{2} t \\
0 & 1
\end{array}\right)
$$

(w.l.o.g. we assume $\lambda_{1}=0$ and $\lambda_{2} \neq 0$ ). In other words, in the basis in which $K$ is diagonal, $S(t)$ has Jordan normal form and is a shear matrix, compare Eq. (37b). Denoting the eigenvectors of $K$ by $\left|\lambda_{1}\right\rangle$ and $\left|\lambda_{2}\right\rangle$, we write $S(t)=\lambda_{2} t\left|\lambda_{1}\right\rangle\left\langle\lambda_{2}|+| \lambda_{1}\right\rangle\left\langle\lambda_{1}|+| \lambda_{2}\right\rangle\left\langle\lambda_{2}\right|$, such that for late times $C(t)=\left(\lambda_{2} t\right)^{2} C_{\lambda_{2}}\left|\lambda_{1}\right\rangle\left\langle\lambda_{1}\right|+\mathcal{O}(t)$, where $C_{\lambda_{2}}=\left\langle\lambda_{2}|C(0)| \lambda_{2}\right\rangle$. By the same reasoning as above, we conclude that $C(t)$ has a quadratically increasing and inversely quadratic decreasing eigenvalue whose eigenvectors approach $\left|\lambda_{1}\right\rangle$ and $\left|\lambda_{2}\right\rangle$ for late times, respectively.

\section{F Wigner function of Gaussian density}

In this Appendix we compute the Wigner function $W_{\rho}$ of a Gaussian density matrix $\rho$ on $\mathrm{E}^{2}\left(\mathbb{R}^{n}\right)$. This is a generalization of Proposition 242 in [86]. The final result is Eq. (45).

Let the kernel of $\rho$ be

$$
\rho\left(x, x^{\prime}\right)=\sqrt{\frac{\operatorname{det}\left(X_{11}+X_{12}\right)}{\pi^{n}}} \exp \left(-\frac{1}{2}\left(x, x^{\prime}\right) \Gamma\left(x, x^{\prime}\right)\right),
$$

where the $2 n$ by $2 n$, symmetric, inverse covariance matrix $\Gamma=X+i Y$ has positive definite real part $\Re \Gamma=X>0$, and

$$
\begin{array}{ll}
X_{11}=X_{22} \text { symmetric, } & Y_{11}=-Y_{22} \text { symmetric, } \\
X_{12}=X_{21} \text { symmetric, } & Y_{12}=-Y_{21} \text { antisymmetric }
\end{array}
$$


( $X_{i j}$ denoting $n$ by $n$ blocks of the two by two block matrix $X$, and similarly for $Y$ ). Eq. (44) is a consequence of Hermiticity of $\rho$, i.e. $\rho\left(x, x^{\prime}\right)=\rho\left(x^{\prime}, x\right)^{*}$, and symmetry of $\Gamma$. The factor $\left[\operatorname{det}\left(X_{11}+X_{12}\right) / \pi^{n}\right]^{1 / 2}$ normalizes the trace $\operatorname{Tr} \rho=\int \rho(x, x) d^{n} x$ to unity (positivity of $X$ guarantees positivity of the radicand).

A lengthy, but straightforward calculation of $W_{\rho}(x, p)=\int d^{n} \eta \rho\left(x-\frac{\eta}{2}, x+\frac{\eta}{2}\right) e^{i p \eta}$, using the Fourier transform of the Gaussian $\int d^{n} x[\operatorname{det}(2 \pi C)]^{-1 / 2} \exp \left(-\frac{1}{2} x C^{-1} x\right) e^{-i p x}=\exp \left(-\frac{1}{2} p C p\right)$, yields

$$
W_{\rho}(z)=2^{n}\left(\operatorname{det} X_{+} / \operatorname{det} X_{-}\right)^{1 / 2} \exp (-z G z)
$$

where

$$
\begin{aligned}
G & =\left(\begin{array}{cc}
X_{+}+Y_{-} X_{-}^{-1} Y_{+} & Y_{-} X_{-}^{-1} \\
X_{-}^{-1} Y_{+} & X_{-}^{-1}
\end{array}\right), \\
G^{-1} & =\left(\begin{array}{cc}
X_{+}^{-1} & -X_{+}^{-1} Y_{-} \\
-Y_{+} X_{+}^{-1} & X_{-}+Y_{+} X_{+}^{-1} Y_{-}
\end{array}\right),
\end{aligned}
$$

introducing the short hand notation $X_{ \pm}=\left(X_{11} \pm X_{12}\right)$, and $Y_{ \pm}=\left(Y_{11} \pm Y_{12}\right)$, such that $X_{ \pm}^{T}=X_{ \pm}$, and $Y_{ \pm}^{T}=Y_{\mp}$, according to (44). The normalization is $\int W_{\rho}(z) d^{2 n} z /(2 \pi)^{n}=1$. In other words, $W_{\rho}$ is (proportional to) a Gaussian with covariance matrix $\Sigma=G^{-1} / 2$.

In the special case when $\Gamma_{12}=0$, the kernel $\rho\left(x, x^{\prime}\right)$ factorizes and is the a rank one projection (pure state) onto the $L^{2}$-normalized Gaussian function $\psi(x)=(\pi)^{-n / 4}\left(\operatorname{det} X_{11}\right)^{1 / 4} \exp \left(-\frac{1}{2} x\left(X_{11}+\right.\right.$ $\left.i Y_{11}\right) x$ ). Then, (45) agrees with Proposition 242 in [86]. Moreover, if $\Gamma_{12}=0, G$ is positive definite, symplectic, and $G=S^{T} S$, where

$$
S=\left(\begin{array}{cc}
X_{11}^{1 / 2} & 0 \\
X_{11}^{-1 / 2} Y_{11} & X_{11}^{-1 / 2}
\end{array}\right)
$$

is symplectic. That is, the symplectic spectrum of $G$ is unity.

\section{G Replica trick}

The von Neumann entanglement entropy of Gaussian states was computed by means of the replica trick in $[91,92]$. For the sake of completeness, the computation is reviewed in our notation. The final result is given in Eqs. (50) and (51).

The replica trick allows to compute the von Neumann entropy as the derivative

$$
S_{\mathrm{vN}}\left(\rho_{A}\right)=-\left.\partial_{n}\right|_{n=1} \operatorname{Tr}\left(\rho_{A}^{n}\right) .
$$

A variant of this formula,

$$
S_{\mathrm{vN}}\left(\rho_{A}\right)=\left(-\left.\partial_{n}\right|_{n=1}+1\right) \log \operatorname{Tr}\left(\rho_{A}^{n}\right),
$$

has the advantage that $\rho_{A}$ in Eq. (47) does not need to be normalized. The idea is to find an easy explicit symbolic expression of $\operatorname{Tr} \rho_{A}^{n}$ in $n$ and then differentiate this expression w.r.t. $n$. Once $\operatorname{Tr}\left(\rho_{A}^{n}\right)$ is computed for $n=1,2,3, \ldots$, one also knows all the other Rényi entropies $S_{n}=\log \left[\operatorname{Tr}\left(\rho_{A}^{n}\right)\right] /(1-n)$. 
Let $\psi$ be a Gaussian wave function on the bipartite Hilbert space $L^{2}(\mathbb{R}) \otimes L^{2}(\mathbb{R})$ (the derivation can be generalized to $L^{2}\left(\mathbb{R}^{d}\right) \otimes L^{2}\left(\mathbb{R}^{d}\right)$ ),

$$
\psi\left(x_{A}, x_{B}\right) \sim \exp \left[-\frac{1}{2}\left(\begin{array}{ll}
x_{A} & x_{B}
\end{array}\right) \Gamma^{A B}\left(\begin{array}{l}
x_{A} \\
x_{B}
\end{array}\right)\right],
$$

with complex valued, symmetric, two by two inverse covariance $\Gamma^{A B}$. The reduced density matrix

$$
\rho_{A}(x, y) \sim \exp \left[-\frac{1}{2}\left(\begin{array}{ll}
x & y
\end{array}\right) \Gamma^{A}\left(\begin{array}{l}
x \\
y
\end{array}\right)\right]
$$

is again Gaussian with inverse covariance [11]

$$
\begin{aligned}
& \Gamma_{11}^{A}=\Gamma_{11}^{A B}-\frac{1}{2} \Gamma_{12}^{A B}\left(\Re \Gamma_{22}^{A B}\right)^{-1} \Gamma_{12}^{A B}, \\
& \Gamma_{12}^{A}=-\frac{1}{2} \Gamma_{12}^{A B}\left(\Re \Gamma_{22}^{A B}\right)^{-1} \overline{\Gamma_{12}^{A B},}
\end{aligned}
$$

and, due to the hermiticity of $\rho_{A}, \Gamma_{2,1}^{A}=\overline{\Gamma_{1,2}^{A}}$, and $\Gamma_{2,2}^{A}=\overline{\Gamma_{1,1}^{A}}$. The trace of $\rho_{A}^{n}$ is then proportional to the integral over the $n$ dimensional Gaussian

$$
\operatorname{Tr}\left(\rho_{A}^{n}\right) \sim \int d^{n} x \exp \left[-\frac{1}{2} x M x\right] \sim \operatorname{det}(M)^{-1 / 2}
$$

with $M$ being the circulant $n$ by $n$ matrix

$$
M=\left(\begin{array}{cccccc}
\Gamma_{11}^{A}+\Gamma_{22}^{A} & \Gamma_{12}^{A} & 0 & \cdots & 0 & \Gamma_{21}^{A} \\
\Gamma_{21}^{A} & \ddots & \ddots & \ddots & & 0 \\
0 & \ddots & & & \ddots & \vdots \\
\vdots & \ddots & & & \ddots & 0 \\
0 & & \ddots & \ddots & \ddots & \Gamma_{12}^{A} \\
\Gamma_{12}^{A} & 0 & \cdots & 0 & \Gamma_{21}^{A} & \Gamma_{11}^{A}+\Gamma_{22}^{A}
\end{array}\right) .
$$

This matrix is not symmetric, but as it is contracted with a symmetric tensor in the expression $M_{i j} x_{i} x_{j}$, we may replace $M$ by its symmetric part $\tilde{M}=\left(M+M^{T}\right) / 2$,

$$
\tilde{M}=\left(\begin{array}{cccccc}
2 \Re \Gamma_{11}^{A} & \Re \Gamma_{12}^{A} & 0 & \ldots & 0 & \Re \Gamma_{12}^{A} \\
\Re \Gamma_{12}^{A} & \ddots & \ddots & \ddots & & 0 \\
0 & \ddots & & & \ddots & \vdots \\
\vdots & \ddots & & & \ddots & 0 \\
0 & & \ddots & \ddots & \ddots & \Re \Gamma_{12}^{A} \\
\Re \Gamma_{12}^{A} & 0 & \cdots & 0 & \Re \Gamma_{12}^{A} & 2 \Re \Gamma_{11}^{A}
\end{array}\right)
$$

(where we have used $\Gamma_{2,1}^{A}=\overline{\Gamma_{1,2}^{A}}, \Gamma_{2,2}^{A}=\overline{\Gamma_{1,1}^{A}}$ ). The integral in (49) is thus proportional to $\operatorname{det}(\tilde{M})^{-1 / 2}$. The determinant is known to be [93]

$$
\begin{aligned}
\operatorname{det}(\tilde{M}) & =\prod_{j=0}^{n-1}\left[2 \Re \Gamma_{11}^{A}+2 \Re \Gamma_{12}^{A} \cos (2 \pi j / n)\right] \\
& =\left(2 \Re \Gamma_{11}^{A}\right)^{n} \prod_{j=0}^{n-1}\left[1+\Re \Gamma_{12}^{A} / \Re \Gamma_{11}^{A} \cos (2 \pi j / n)\right] .
\end{aligned}
$$


We only need to compute $\operatorname{det}(\tilde{M})$ modulo factors of $n$-th power. This is because $\rho_{A}$ in Eq. (47) does not need to be normalized and rescaling of $\rho_{A}$ leads to factors of $n$-th power in $\operatorname{Tr}\left(\rho_{A}^{n}\right)$ and hence in $\operatorname{det}(\tilde{M})$. Thus, we may drop all global factors of $n$-th power in $\operatorname{det} \tilde{M}$, which we indicate by writing $\sim$ instead of the equality sign. Now, we define $\xi$ by $\Re \Gamma_{12}^{A} / \Re \Gamma_{11}^{A}=-2 \xi /\left(1+\xi^{2}\right)$ and use the identity $\prod_{j=0}^{n-1}\left[1+\xi^{2}-2 \xi \cos (2 \pi j / n)\right]=\left(1-\xi^{n}\right)^{2}$ to obtain

$$
\operatorname{det}(\tilde{M}) \sim\left(1-\xi^{n}\right)^{2} .
$$

The von Neumann entropy follows from Eq. (47)

$$
S_{\mathrm{vN}}=-\log (1-\xi)-\frac{\xi}{1-\xi} \log (\xi) .
$$

It is not obvious, but $0<\xi<1$ (to be more precise, only $\xi_{-}$of the two solutions $\xi_{ \pm}=$ $-\Re \Gamma_{11}^{A} / \Re \Gamma_{12}^{A} \pm \sqrt{\left(\Re \Gamma_{11}^{A} / \Re \Gamma_{12}^{A}\right)^{2}-1}$ obeys this constraint), so that the above expression is always real and positive. The other Rényi entropies are given by

$$
S_{n}=\frac{1}{1-n} \log \frac{(1-\xi)^{n}}{1-\xi^{n}}
$$

Eqs. (50) and (51) are equivalent to Eqs. (23) and (22), respectively, upon the identification $\xi=\exp (-\omega)=(2 \lambda-1) /(2 \lambda+1)$.

As a corollary of the result (51), we obtain the spectrum

$$
\operatorname{Spec}\left(\rho_{A}\right)=\left\{(1-\xi) \xi^{j}: j \in \mathbb{N}_{0}\right\}
$$

of the reduced density matrix $\rho_{A}$. This equation follows from comparing (51) with $\frac{1}{1-n} \log \sum_{j} \lambda_{j}^{n}$, where $\lambda_{j}$ denotes the sequence of eigenvalues of $\rho_{A}$. The equations

$$
\sum_{j=0} \lambda_{j}^{n}=\frac{(1-\xi)^{n}}{1-\xi^{n}}
$$

for all positive integers $n$, are solved by $\lambda_{j}=(1-\xi) \xi^{j}$. Eq. (52) is consistent with (20).

\section{H Spin squeezing and entanglement}

In this appendix we compute $\lambda$ (cf. Eq. (24)) as a function $\xi_{S}$. In the sequel, we write $g_{2}$ and $-\theta_{2}$ for the real and imaginary part of $f_{2}=g_{2}-i \theta_{2}$. The covariance matrix $C^{\perp}$ (cf. Eq. (25)) is Hermitian and its real part is

$$
\Re C^{\perp}=\frac{1}{2 g_{2} N}\left(\begin{array}{cc}
\sin ^{-2} \theta & \frac{\theta_{2}}{2} \\
\frac{\theta_{2}}{2} & \frac{\theta_{2}^{2}+g_{2}^{2}}{4} \sin ^{2} \theta
\end{array}\right)+\mathcal{O}\left(1 / N^{2}\right) .
$$

The leading order of the determinant of $C^{\perp}$ is $(4 N)^{-2}$ (independent of $g_{2}$ and $\theta_{2}$ ). This means that the uncertainty between the magnetization in the two directions of the eigenvectors of $C^{\perp}$ is minimized to leading order,

$$
\operatorname{det} C^{\perp} \geq \frac{1}{4 N^{2}}|\langle\mathbf{S} \cdot \hat{\Omega}\rangle|^{2}=\frac{1}{(4 N)^{2}}+\mathcal{O}\left(1 / N^{3}\right) .
$$


In the special case, when the eigenvalues of $C^{\perp}$ are identical, the uncertainty between the magnetization in any two directions in the $\hat{\Omega}_{1}^{\perp} \hat{\Omega}_{2}^{\perp}$ plane is minimized. This is the situation of coherent states which are non-entangled (see below).

The determinant and the trace of $C^{\perp}$ are invariant under rotations of the Bloch sphere, i.e. changes of the quantization axis. Determinant and trace are the only two independent basis independent properties of a two by two matrix. As the leading order of the determinant is constant, the entanglement entropy can only depend on the trace. In fact, Eq. (24) can be rewritten as

$$
\lambda=\sqrt{\frac{1}{4}+\alpha \beta\left(N \operatorname{Tr} C^{\perp}-\frac{1}{2}\right)} .
$$

Since $\operatorname{det} C^{\perp}=(4 N)^{-2}$, the trace of $C^{\perp}$ is bounded from below by $(2 N)^{-1}$. More precisely, $\operatorname{Tr} C^{\perp}=(2 N)^{-1}$ if and only if both eigenvalues of $C^{\perp}$ are identical to $(4 N)^{-1}$ (coherent states). The Isotropic variance of $(4 N)^{-1}$ at minimal uncertainty is called the standard quantum limit (SQL) $[45,49]$. In this case $\lambda=1 / 2$ and all Rényi entropies vanish (cf. Eq. (22)). This is also consistent with the observation that the symplectic spectrum of the covariance of a Gaussian pure state is one half, see the discussion around Eq. (46).

Let $\lambda_{1}$ and $\lambda_{2}$ be the eigenvalues of $C^{\perp}$ with $\lambda_{1} \leq \lambda_{2}$ and $\lambda_{1} \lambda_{2}=(4 N)^{-2}$, then (cf. Eq. (26))

$$
\xi_{S}^{2}=\sqrt{\lambda_{1} / \lambda_{2}}=4 N \lambda_{1}=2 N \operatorname{Tr} C^{\perp}-\sqrt{\left(2 N \operatorname{Tr} C^{\perp}\right)^{2}-1},
$$

which, together with Eq. (54), gives $\lambda$ as a function of $\xi_{S}^{2}$. The von Neumann entanglement entropy (and any other Rényi entanglement entropy) is thus an explicit function of the squeezing parameter $\xi_{S}^{2}$.

Details on the computation of $C^{\perp}$ : Equation (53) follows from the lengthy calculation of

$$
\begin{aligned}
\left\langle S_{x}, S_{x}\right\rangle_{c}= & +(\cos \phi \cot \theta)^{2} \frac{1}{2 g_{2} N}+\cos \theta \cos \phi \sin \phi \frac{\theta_{2}}{2 g_{2} N} \\
& +\left(\frac{1}{2} \sin \phi \sin \theta\right)^{2} \frac{\theta_{2}^{2}+g_{2}^{2}}{2 g_{2} N}+\mathcal{O}\left(1 / N^{2}\right), \\
\left\langle S_{y}, S_{y}\right\rangle_{c}= & +(\sin \phi \cot \theta)^{2} \frac{1}{2 g_{2} N}-\cos \theta \cos \phi \sin \phi \frac{\theta_{2}}{2 g_{2} N} \\
& +\left(\frac{1}{2} \cos \phi \sin \theta\right)^{2} \frac{\theta_{2}^{2}+g_{2}^{2}}{2 g_{2} N}+\mathcal{O}\left(1 / N^{2}\right), \\
\left\langle S_{z}, S_{z}\right\rangle_{c}= & +\frac{1}{2 g_{2} N}+\mathcal{O}\left(1 / N^{2}\right), \\
\Re\left\langle S_{x}, S_{y}\right\rangle_{c}= & +(\cot \theta)^{2} \sin \phi \cos \phi \frac{1}{2 g_{2} N}+\frac{1}{2} \cos \theta\left(\sin ^{2} \phi-\cos ^{2} \phi\right) \frac{\theta_{2}}{2 g_{2} N} \\
& -\left(\frac{1}{2} \sin \theta\right)^{2} \sin \phi \cos \phi \frac{\theta_{2}^{2}+g_{2}^{2}}{2 g_{2} N}+\mathcal{O}\left(1 / N^{2}\right), \\
\Re\left\langle S_{x}, S_{z}\right\rangle_{c}= & -\cos \phi \cot \theta \frac{1}{2 g_{2} N}-\frac{1}{2} \sin \phi \sin \theta \frac{\theta_{2}}{2 g_{2} N}+\mathcal{O}\left(1 / N^{2}\right), \\
\Re\left\langle S_{y}, S_{z}\right\rangle_{c}= & -\sin \phi \cot \theta \frac{1}{2 g_{2} N}+\frac{1}{2} \cos \phi \sin \theta \frac{\theta_{2}}{2 g_{2} N}+\mathcal{O}\left(1 / N^{2}\right) .
\end{aligned}
$$


The results (55) to (60) can be obtained by carefully approximating the expectation values in the state $\psi \asymp e^{-N f(s)}$ to next to leading order in a saddle point approximation.

\section{References}

[1] A. Einstein, B. Podolsky and N. Rosen, Can quantum-mechanical description of physical reality be considered complete?, Phys. Rev. 47, 777 (1935), doi:10.1103/PhysRev.47.777.

[2] E. Schrödinger, Discussion of probability relations between separated systems, Mathematical Proceedings of the Cambridge Philosophical Society 31, 555 (1935), doi:10.1017/S0305004100013554.

[3] S. R. White, Density matrix formulation for quantum renormalization groups, Phys. Rev. Lett. 69, 2863 (1992), doi:10.1103/PhysRevLett.69.2863.

[4] A. J. Daley, C. Kollath, U. Schollwöck and G. Vidal, Time-dependent density-matrix renormalization-group using adaptive effective hilbert spaces, Journal of Statistical Mechanics: Theory and Experiment 2004(04), P04005 (2004), doi:10.1088/1742$5468 / 2004 / 04 / \mathrm{p} 04005$.

[5] U. Schollwöck, The density-matrix renormalization group, Rev. Mod. Phys. 77, 259 (2005), doi:10.1103/RevModPhys.77.259.

[6] U. Schollwöck, The density-matrix renormalization group in the age of matrix product states, Annals of Physics 326(1), 96 (2011), doi:https://doi.org/10.1016/j.aop.2010.09.012, January 2011 Special Issue.

[7] J. Eisert and T. J. Osborne, General entanglement scaling laws from time evolution, Phys. Rev. Lett. 97, 150404 (2006), doi:10.1103/PhysRevLett.97.150404.

[8] J. L. Cardya, Entanglement entropy in extended quantum systems, The European Physical Journal B 64(3), 321 (2008), doi:10.1140/epjb/e2008-00102-5.

[9] P. Calabrese and J. Cardy, Entanglement entropy and conformal field theory, Journal of Physics A: Mathematical and Theoretical 42(50), 504005 (2009), doi:10.1088/1751$8113 / 42 / 50 / 504005$.

[10] H. Kim and D. A. Huse, Ballistic spreading of entanglement in a diffusive nonintegrable system, Phys. Rev. Lett. 111, 127205 (2013), doi:10.1103/PhysRevLett.111.127205.

[11] R. G. Unanyan and M. Fleischhauer, Entanglement dynamics in harmonic-oscillator chains, Phys. Rev. A 89, 062330 (2014), doi:10.1103/PhysRevA.89.062330.

[12] M. Srednicki, Entropy and area, Phys. Rev. Lett. 71, 666 (1993), doi:10.1103/PhysRevLett.71.666.

[13] P. Calabrese and J. Cardy, Entanglement entropy and quantum field theory, Journal of Statistical Mechanics: Theory and Experiment 2004(06), P06002 (2004), doi:10.1088/1742-5468/2004/06/p06002. 
[14] M. B. Plenio, J. Eisert, J. Dreißig and M. Cramer, Entropy, entanglement, and area: Analytical results for harmonic lattice systems, Phys. Rev. Lett. 94, 060503 (2005), doi:10.1103/PhysRevLett.94.060503.

[15] E. Fradkin and J. E. Moore, Entanglement entropy of $2 d$ conformal quantum critical points: Hearing the shape of a quantum drum, Phys. Rev. Lett. 97, 050404 (2006), doi:10.1103/PhysRevLett.97.050404.

[16] J. Eisert, M. Cramer and M. B. Plenio, Colloquium: Area laws for the entanglement entropy, Rev. Mod. Phys. 82, 277 (2010), doi:10.1103/RevModPhys.82.277.

[17] W. W. Ho and D. A. Abanin, Entanglement dynamics in quantum many-body systems, Phys. Rev. B 95, 094302 (2017), doi:10.1103/PhysRevB.95.094302.

[18] J. Schachenmayer, B. P. Lanyon, C. F. Roos and A. J. Daley, Entanglement growth in quench dynamics with variable range interactions, Phys. Rev. X 3, 031015 (2013), doi:10.1103/PhysRevX.3.031015.

[19] R. Singh, R. Moessner and D. Roy, Effect of long-range hopping and interactions on entanglement dynamics and many-body localization, Phys. Rev. B 95, 094205 (2017), doi:10.1103/PhysRevB.95.094205.

[20] V. Alba and F. Heidrich-Meisner, Entanglement spreading after a geometric quench in quantum spin chains, Phys. Rev. B 90, 075144 (2014), doi:10.1103/PhysRevB.90.075144.

[21] J. H. Bardarson, F. Pollmann and J. E. Moore, Unbounded growth of entanglement in models of many-body localization, Phys. Rev. Lett. 109, 017202 (2012), doi:10.1103/PhysRevLett.109.017202.

[22] M. Serbyn, Z. Papić and D. A. Abanin, Universal slow growth of entanglement in interacting strongly disordered systems, Phys. Rev. Lett. 110, 260601 (2013), doi:10.1103/PhysRevLett.110.260601.

[23] D. A. Huse, R. Nandkishore and V. Oganesyan, Phenomenology of fully many-bodylocalized systems, Phys. Rev. B 90, 174202 (2014), doi:10.1103/PhysRevB.90.174202.

[24] I. Homrighausen, N. O. Abeling, V. Zauner-Stauber and J. C. Halimeh, Anomalous dynamical phase in quantum spin chains with long-range interactions, Phys. Rev. B 96, 104436 (2017), doi:10.1103/PhysRevB.96.104436.

[25] J. Lang, B. Frank and J. C. Halimeh, Dynamical quantum phase transitions: A geometric picture, Phys. Rev. Lett. 121, 130603 (2018), doi:10.1103/PhysRevLett.121.130603.

[26] J. Lang, B. Frank and J. C. Halimeh, Concurrence of dynamical phase transitions at finite temperature in the fully connected transverse-field ising model, Phys. Rev. B 97, 174401 (2018), doi:10.1103/PhysRevB.97.174401.

[27] A. S. Buyskikh, M. Fagotti, J. Schachenmayer, F. Essler and A. J. Daley, Entanglement growth and correlation spreading with variable-range interactions in spin and fermionic tunneling models, Phys. Rev. A 93, 053620 (2016), doi:10.1103/PhysRevA.93.053620. 
[28] S. Pappalardi, A. Russomanno, B. Žunkovič, F. Iemini, A. Silva and R. Fazio, Scrambling and entanglement spreading in long-range spin chains, Phys. Rev. B 98, 134303 (2018), doi:10.1103/PhysRevB.98.134303.

[29] M. Hillery and M. S. Zubairy, Entanglement conditions for two-mode states, Phys. Rev. Lett. 96, 050503 (2006), doi:10.1103/PhysRevLett.96.050503.

[30] C. Pérez-Campos, J. R. González-Alonso, O. Castanos and R. López-Pena, Entanglement and localization of a two-mode bose-einstein condensate, Annals of Physics 325(2), 325 (2010), doi:https://doi.org/10.1016/j.aop.2009.09.015.

[31] H. Hennig, D. Witthaut and D. K. Campbell, Global phase space of coherence and entanglement in a double-well bose-einstein condensate, Phys. Rev. A 86, 051604 (2012), doi:10.1103/PhysRevA.86.051604.

[32] T. Pudlik, H. Hennig, D. Witthaut and D. K. Campbell, Tunneling in the self-trapped regime of a two-well BEC, ArXiv e-prints (2014), 1409.0769.

[33] A. Lerose and S. Pappalardi, Origin of the slow growth of entanglement entropy in long-range interacting systems, arXiv e-prints arXiv:1811.05505 (2018), 1811.05505.

[34] M. Albiez, R. Gati, J. Fölling, S. Hunsmann, M. Cristiani and M. K. Oberthaler, Direct observation of tunneling and nonlinear self-trapping in a single bosonic josephson junction, Phys. Rev. Lett. 95, 010402 (2005), doi:10.1103/PhysRevLett.95.010402.

[35] J. Estève, C. Gross, A. Weller, S. Giovanazzi and M. K. Oberthaler, Squeezing and entanglement in a bose-einstein condensate, Nature 455, 1216 EP (2008), doi:10.1038/nature07332.

[36] J. Vidal, G. Palacios and C. Aslangul, Entanglement dynamics in the lipkin-meshkov-glick model, Phys. Rev. A 70, 062304 (2004), doi:10.1103/PhysRevA.70.062304.

[37] K. R. A. Hazzard, M. van den Worm, M. Foss-Feig, S. R. Manmana, E. G. Dalla Torre, T. Pfau, M. Kastner and A. M. Rey, Quantum correlations and entanglement in far-from-equilibrium spin systems, Phys. Rev. A 90, 063622 (2014), doi:10.1103/PhysRevA.90.063622.

[38] H. Lipkin, N. Meshkov and A. Glick, Validity of many-body approximation methods for a solvable model: (i). exact solutions and perturbation theory, Nuclear Physics 62(2), 188 (1965), doi:http://dx.doi.org/10.1016/0029-5582(65)90862-X.

[39] B. Sciolla and G. Biroli, Dynamical transitions and quantum quenches in mean-field models, Journal of Statistical Mechanics: Theory and Experiment 2011(11), P11003 (2011), doi:10.1088/1742-5468/2011/11/p11003.

[40] M. Jääskeläinen and P. Meystre, Coherence dynamics of two-mode condensates in asymmetric potentials, Phys. Rev. A 73, 013602 (2006), doi:10.1103/PhysRevA.73.013602.

[41] C. Bodet, J. Estève, M. K. Oberthaler and T. Gasenzer, Two-mode bose gas: Beyond classical squeezing, Phys. Rev. A 81, 063605 (2010), doi:10.1103/PhysRevA.81.063605. 
[42] P. Jordan, Der zusammenhang der symmetrischen und linearen gruppen und das mehrkörperproblem, Zeitschrift für Physik 94(7), 531 (1935), doi:10.1007/BF01330618.

[43] J. Schwinger, On angular momentum, USAEC Report NYO-3071 (1952), doi: $10.2172 / 4389568$.

[44] Q. Y. He, M. D. Reid, T. G. Vaughan, C. Gross, M. Oberthaler and P. D. Drummond, Einstein-podolsky-rosen entanglement strategies in two-well bose-einstein condensates, Phys. Rev. Lett. 106, 120405 (2011), doi:10.1103/PhysRevLett.106.120405.

[45] M. Kitagawa and M. Ueda, Squeezed spin states, Phys. Rev. A 47, 5138 (1993), doi:10.1103/PhysRevA.47.5138.

[46] A. Sørensen, L. M. Duan, J. I. Cirac and P. Zoller, Many-particle entanglement with Bose-Einstein condensates, Nature 409(6816), 63 (2001), doi:10.1038/35051038.

[47] G. Tóth, C. Knapp, O. Gühne and H. J. Briegel, Spin squeezing and entanglement, Phys. Rev. A 79, 042334 (2009), doi:10.1103/PhysRevA.79.042334.

[48] G. Vitagliano, I. Apellaniz, I. n. L. Egusquiza and G. Tóth, Spin squeezing and entanglement for an arbitrary spin, Phys. Rev. A 89, 032307 (2014), doi:10.1103/PhysRevA.89.032307.

[49] J. Ma, X. Wang, C. Sun and F. Nori, Quantum spin squeezing, Physics Reports 509(2), 89 (2011), doi:https://doi.org/10.1016/j.physrep.2011.08.003.

[50] A. Smerzi, S. Fantoni, S. Giovanazzi and S. R. Shenoy, Quantum coherent atomic tunneling between two trapped bose-einstein condensates, Phys. Rev. Lett. 79, 4950 (1997), doi:10.1103/PhysRevLett.79.4950.

[51] S. Raghavan, A. Smerzi, S. Fantoni and S. R. Shenoy, Coherent oscillations between two weakly coupled bose-einstein condensates: Josephson effects, $\pi$ oscillations, and macroscopic quantum self-trapping, Phys. Rev. A 59, 620 (1999), doi:10.1103/PhysRevA.59.620.

[52] V. S. Shchesnovich and M. Trippenbach, Fock-space wkb method for the boson josephson model describing a bose-einstein condensate trapped in a double-well potential, Phys. Rev. A 78, 023611 (2008), doi:10.1103/PhysRevA.78.023611.

[53] R. G. Littlejohn, The semiclassical evolution of wave packets, Physics Reports 138(4-5), 193 (1986), doi:http://dx.doi.org/10.1016/0370-1573(86)90103-1.

[54] E. Heller, title, In M.-J. Giannoni, A. Voros and J. Zinn-Justin, eds., Chaos and Quantum Physics, Les Houches Lecture Series LII. North-Holland, Amsterdam (1991).

[55] J. H. V. Vleck, The correspondence principle in the statistical interpretation of quantum mechanics, Proceedings of the National Academy of Sciences of the United States of America 14(2), 178 (1928).

[56] J. B. Keller, Corrected bohr-sommerfeld quantum conditions for nonseparable systems, Annals of Physics 4(2), 180 (1958), doi:https://doi.org/10.1016/0003-4916(58)90032-0. 
[57] P. Morse and H. Feshbach, Methods of theoretical physics, No. Bd. 1 in International series in pure and applied physics. McGraw-Hill (1953).

[58] R. S. Ellis, An overview of the theory of large deviations and applications to statistical mechanics, Scandinavian Actuarial Journal 1995(1), 97 (1995), doi:10.1080/03461238.1995.10413952, http://dx.doi.org/10.1080/03461238.1995. 10413952.

[59] H. Touchette, The large deviation approach to statistical mechanics, Physics Reports 478(1-3), 1 (2009), doi:http://dx.doi.org/10.1016/j.physrep.2009.05.002.

[60] E. Wigner, On the quantum correction for thermodynamic equilibrium, Phys. Rev. 40, 749 (1932), doi:10.1103/PhysRev.40.749.

[61] J. E. Moyal, Quantum mechanics as a statistical theory, Mathematical Proceedings of the Cambridge Philosophical Society 45(1), 99 (1949), doi:10.1017/S0305004100000487.

[62] M. V. Berry, Semi-classical mechanics in phase space: A study of wigner's function, Philosophical Transactions of the Royal Society of London A: Mathematical, Physical and Engineering Sciences 287(1343), 237 (1977), doi:10.1098/rsta.1977.0145.

[63] M. Hillery, R. O'Connell, M. Scully and E. Wigner, Distribution functions in physics: Fundamentals, Physics Reports 106(3), 121 (1984), doi:https://doi.org/10.1016/03701573(84)90160-1.

[64] A. Polkovnikov, Phase space representation of quantum dynamics, Annals of Physics 325(8), 1790 (2010), doi:http://dx.doi.org/10.1016/j.aop.2010.02.006.

[65] E. J. Heller, Wigner phase space method: Analysis for semiclassical applications, The Journal of Chemical Physics 65(4), 1289 (1976), doi:10.1063/1.433238, https://doi. org/10.1063/1.433238.

[66] E. J. Heller, Time-dependent approach to semiclassical dynamics, The Journal of Chemical Physics 62(4), 1544 (1975), doi:http://dx.doi.org/10.1063/1.430620.

[67] E. J. Heller, Wavepacket path integral formulation of semiclassical dynamics, Chemical Physics Letters 34(2), 321 (1975), doi:http://dx.doi.org/10.1016/0009-2614(75)85284-5.

[68] E. J. Heller, Classical s-matrix limit of wave packet dynamics, The Journal of Chemical Physics 65(11), 4979 (1976), doi:http://dx.doi.org/10.1063/1.432974.

[69] D. Huber and E. J. Heller, Generalized gaussian wave packet dynamics, The Journal of Chemical Physics 87(9), 5302 (1987), doi:http://dx.doi.org/10.1063/1.453647.

[70] D. Huber, E. J. Heller and R. G. Littlejohn, Generalized gaussian wave packet dynamics, schroedinger equation, and stationary phase approximation, The Journal of Chemical Physics 89(4), 2003 (1988), doi:http://dx.doi.org/10.1063/1.455714.

[71] M. A. M. de Aguiar, M. Baranger, L. Jaubert, F. Parisio and A. D. Ribeiro, Semiclassical propagation of wavepackets with complex and real trajectories, Journal of Physics A: Mathematical and General 38(21), 4645 (2005), doi:10.1088/0305-4470/38/21/010. 
[72] F. Parisio and M. A. M. de Aguiar, A regular semiclassical approximation for the propagation of wave packets with complex trajectories, Journal of Physics A: Mathematical and General 38(42), 9317 (2005), doi:10.1088/0305-4470/38/42/011.

[73] R. N. P. Maia, F. Nicacio, R. O. Vallejos and F. Toscano, Semiclassical propagation of gaussian wave packets, Phys. Rev. Lett. 100, 184102 (2008), doi:10.1103/PhysRevLett.100.184102.

[74] R. Schubert, R. O. Vallejos and F. Toscano, How do wave packets spread? time evolution on ehrenfest time scales, Journal of Physics A: Mathematical and Theoretical 45(21), 215307 (2012), doi:10.1088/1751-8113/45/21/215307.

[75] M. V. Berry, Evolution of semiclassical quantum states in phase space, Journal of Physics A: Mathematical and General 12(5), 625 (1979), doi:10.1088/0305-4470/12/5/012.

[76] K. Hepp, The classical limit for quantum mechanical correlation functions, Communications in Mathematical Physics 35(4), 265 (1974), doi:10.1007/BF01646348.

[77] W. H. Zurek, Decoherence, chaos, quantum-classical correspondence, and the algorithmic arrow of time, Physica Scripta T76(1), 186 (1998), doi:10.1238/physica.topical.076a00186.

[78] J. I. Latorre, R. Orús, E. Rico and J. Vidal, Entanglement entropy in the lipkin-meshkovglick model, Phys. Rev. A 71, 064101 (2005), doi:10.1103/PhysRevA.71.064101.

[79] T. Barthel, S. Dusuel and J. Vidal, Entanglement entropy beyond the free case, Phys. Rev. Lett. 97, 220402 (2006), doi:10.1103/PhysRevLett.97.220402.

[80] T. Holstein and H. Primakoff, Field dependence of the intrinsic domain magnetization of a ferromagnet, Phys. Rev. 58, 1098 (1940), doi:10.1103/PhysRev.58.1098.

[81] R. G. Unanyan, C. Ionescu and M. Fleischhauer, Many-particle entanglement in the gaped antiferromagnetic lipkin model, Phys. Rev. A 72, 022326 (2005), doi:10.1103/PhysRevA.72.022326.

[82] M. Filippone, S. Dusuel and J. Vidal, Quantum phase transitions in fully connected spin models: An entanglement perspective, Phys. Rev. A 83, 022327 (2011), doi:10.1103/PhysRevA.83.022327.

[83] J. Wilms, J. Vidal, F. Verstraete and S. Dusuel, Finite-temperature mutual information in a simple phase transition, Journal of Statistical Mechanics: Theory and Experiment 2012(01), P01023 (2012), doi:10.1088/1742-5468/2012/01/p01023.

[84] M. G. Nezhadhaghighi and M. A. Rajabpour, Entanglement dynamics in short- and long-range harmonic oscillators, Phys. Rev. B 90, 205438 (2014), doi:10.1103/PhysRevB.90.205438.

[85] F. Bayen, M. Flato, C. Fronsdal, A. Lichnerowicz and D. Sternheimer, Quantum mechanics as a deformation of classical mechanics, Letters in Mathematical Physics 1(6), 521 (1977), doi:10.1007/BF00399745. 
[86] M. de Gosson, Symplectic Methods in Harmonic Analysis and in Mathematical Physics, Pseudo-Differential Operators. Springer Basel, ISBN 9783764399924 (2011).

[87] A. S. Holevo, M. Sohma and O. Hirota, Capacity of quantum gaussian channels, Phys. Rev. A 59, 1820 (1999), doi:10.1103/PhysRevA.59.1820.

[88] V. Giovannetti, S. Lloyd and L. Maccone, Quantum-enhanced measurements: Beating the standard quantum limit, Science 306(5700), 1330 (2004), doi:10.1126/science.1104149, http://science.sciencemag.org/content/306/5700/1330.full.pdf.

[89] M. Nielsen and I. Chuang, Quantum Computation and Quantum Information: 10th Anniversary Edition, Cambridge University Press, ISBN 9781139495486 (2010).

[90] F. Verhulst, Nonlinear Differential Equations and Dynamical Systems, Universitext. Springer Berlin Heidelberg, ISBN 9783642614538 (2012).

[91] L. Bombelli, R. K. Koul, J. Lee and R. D. Sorkin, Quantum source of entropy for black holes, Phys. Rev. D 34, 373 (1986), doi:10.1103/PhysRevD.34.373.

[92] C. Callan and F. Wilczek, On geometric entropy, Physics Letters B 333(1), 55 (1994), doi:https://doi.org/10.1016/0370-2693(94)91007-3.

[93] A. Boettcher and S. Grudsky, Spectral Properties of Banded Toeplitz Matrices, Other Titles in Applied Mathematics. Society for Industrial and Applied Mathematics, ISBN 9780898717853 (2005). 


\section{Part II}

\section{Publication Ref. [2]}





\section{Dynamical Phase Transitions}

The publication [2] investigates the connection between two types of DPTs in the FC-TFIM. To distinguish both types, we follow the same nomenclature as in $[2]$ and refer to them as dynamical phase transition of type one (DPT-I) and dynamical phase transition of type two (DPT-II). On the one hand, DPT-I are defined as non-analytic behavior of infinite time averages of observables (typically these observables are viewed as order parameters) 60, 81 as a function of the quench parameters (typically viewed as control parameters). As such, DPT-I can be depicted as special points in a dynamical phase diagram whose axes labels are the pre- and post-quench parameters. In [81] DPT-I were studied in the FC-TFIM, showing that the transverse fields at which a dynamical transition occurs does not coincide with the underlying equilibrium phase transition. Its consequences for the order parameter variance and the entanglement dynamics is discussed in chapter 3 of this thesis. On the other hand, DPT-II are defined as non-analytic behavior of the fidelity rate function as a function of time [59].

In 147] Oppenheimer describes analogy as an important tool of scientific reasoning. By analogy he means the structural similarity of concepts and ideas of different fields of study, and also within the same field of study. An exemplification of this definition is the analogy between equilibrium phase transition and DPT. The structural similarity of both these concepts is the sudden change in behavior of one quantity under the influence of another.

\begin{tabular}{ccc}
$\begin{array}{c}\text { equilibrium phase } \\
\text { transition }\end{array}$ & DPT-I & DPT-II \\
\hline $\begin{array}{c}\text { control parameter } \\
\text { (e.g. } \beta)\end{array}$ & quench parameter & time \\
\hline $\begin{array}{c}\text { order parameter } \\
\text { (e.g. free energy) }\end{array}$ & $\begin{array}{c}\text { time average of } \\
\text { observable }\end{array}$ & $\begin{array}{c}\text { Loschmidt rate } \\
\text { function }\end{array}$
\end{tabular}

Ref. 2 is joint work of the author of this thesis together with Nils Abeling, Valentin ZaunerStauber, and Jad Halimeh. Jad Halimeh and Valentin Zauner-Stauber initiated the project building on their numerical observations of the fidelity dynamics in a long range model in Ref. [148]. In this publication they conjecture a connection between DPT-I and DPT-II. The connection was then corroborated for the FC-TFIM by discussing the exact diagonalization (ED) data by all four authors of [2]. Together with Nils Abeling, the author of this thesis produced the ED results and the semiclassical interpretation.

The following sections leading to Sec. 4.5 discuss the DPT-II in more depth than the publication 2], and contain independent results found by the author of this thesis. In particular, a heuristic semiclassical phase space interpretation of DPT-II in FC-TFIM is given in Sec. 4.2. Thereafter, in Sec. 4.3, DPT-II is discussed on the basis of a semiclassical expansion of the density of states. This work was also presented at the DPG Frühjahrstagung in 2015 by the author of this treatise, but never published in a journal. Later, similar results were published in 149, 150]. 


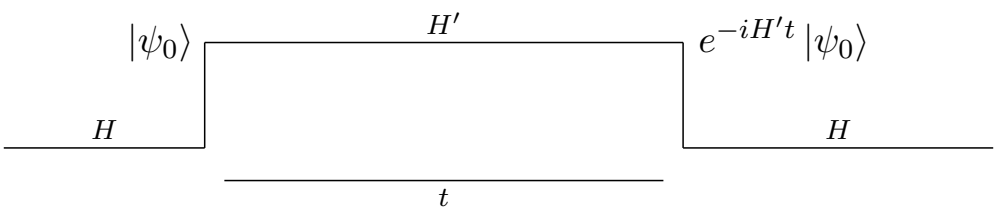

Figure 4.1: Schematic quench of a double quench. At initial time the state $\left|\psi_{0}\right\rangle$ is prepared in the ground state of the pre-quench Hamiltonian $H$. The state evolves under the postquench Hamiltonian $H^{\prime}$ for time $t$. In general, the final state $\left|\psi_{t}\right\rangle$ is not an eigenstate of $H$ and has excess energy in the bulk of the pre-quench spectrum.

\subsection{Introduction}

The notion of DPT-II was established in the seminal paper [59] by Heyl, Polkovnikov, and Kehrein. In this reference, the rate function $f(t)$ of the Loschmidt return amplitude

$$
\langle\psi(0) \mid \psi(t)\rangle \asymp \exp [-N f(t)]
$$

( $N$ being the system size) of a time evolved many particle quantum state in the one-dimensional transverse field Ising model (1D-TFIM) after a quantum-quench was investigated. In analogy to equilibrium phase transitions, the rate function is referred to as the dynamical free energy density, and non-analytic behavior in $f(t)$ as a function of time were coined ${ }^{1}$ DPT-II. The analogy to equilibrium physics becomes more precise by Wick rotating $i t \mapsto \beta$ and analytic continuation, such that $\left\langle\psi_{0} \mid \psi_{t}\right\rangle \mapsto\left\langle\psi_{0}\left|e^{-\beta H}\right| \psi_{0}\right\rangle=Z(\beta)$ takes the form of a boundary partition function. The authors of [59] found that DPT-II occur only when the quench is across the critical line of the equilibrium phase transition separating the paramagnetic from the ferromagnetic phase. Furthermore, the critical times $t^{*}$, at which $f(t)$ is non-analytic, were identified as Fisher zeros, $Z\left(i t^{*}\right)=0$, of the partition function. This is in close analogy to temperature-driven phase transitions [151.

One connection of DPT-II to a physical measurable quantity is given by the work distribution function in a double quench 152,

$$
P(W, t)=\sum_{n} \delta\left(E_{n}-E_{0}-W\right)\left|\left\langle E_{n} \mid \psi(t)\right\rangle\right|^{2} \asymp \exp [-N r(w, t)],
$$

where the summation index labels the pre-quench eigenstates $\left|E_{n}\right\rangle$, and the time evolution of $|\psi(t)\rangle$ is w.r.t. the post-quench Hamiltonian starting in the pre-quench ground state $\left|\psi_{0}\right\rangle=\left|E_{0}\right\rangle$, cf. 4.1 . $P(W, t) d W$ is the probability that the difference of a measurement of $H$ in $|\psi(t)\rangle$ and in $|\psi(0)\rangle$ lies in $[W, W+d W]$. For $W=0, P(W=0, t)=|\langle\psi(t) \mid \psi(0)\rangle|^{2}$ is the Loschmidt return probability (Loschmidt echo), whose rate function $r(w=0, t)=2 \Re f(t)$ inherits the non-analytic behavior from $f(t)$. Similar to their equilibrium counterpart, DPT-II can only occur in the thermodynamic limit, because all terms in the expansion $\langle\psi(0) \mid \psi(t)\rangle=\sum_{n} e^{-i E_{n} t}\left|\left\langle E_{n} \mid \psi(0)\right\rangle\right|^{2}$ are analytic, such that non-analytic behavior can only occur if the summation is infinite, i.e. an infinite Hilbert space dimension is necessary for DPT-II.

Besides the 1D-TFIM, a plethora of other models, such as the one-dimensional [?, 153 154] and infinite-range [149] $X Y$ model, the antiferromagnetic $X X Z$ chain [155], integrable quantum field theories [156], and topological systems [157,158 were shown to exhibit DPT-II. Moreover, results about DPT-II were extended in multiple ways. First, DPT-II were found in non-integrable one-

\footnotetext{
${ }^{1}$ In Ref. 59 they were simply called dynamical phase transition (DPT). But, as mentioned above, we want to avoid confusion with a different kind of dynamical phase transition, and hence use the acronym DPT-II instead.
} 
dimensional systems [159, 160], and long-range systems [148,161]. Second, models in higher spatial dimensions, such as the two-dimensional Kitaev model 162, the two-dimensional transverse field Ising model [163], three-dimensional $O(N)$ model [164], and infinite-dimensional models [2, 149, 150, 165] were shown to exhibit DPT-II. Third, DPT-II were shown to occur in mixed states, in particular, thermal density matrices at non-zero temperature $150,165-167$. The presence and absence of non-analyticities in the rate function of positive work probability has been studied [166]. Fourth, the independence of DPT-II of equilibrium quantum phase transitions was observed in [153, 168].

As mentioned above, DPT-II can only be proven rigorously in the thermodynamic limit. In fact, the Reference [59] uses the exact spectrum of the 1D-TFIM in the thermodynamic limit. Exact solutions of non-trivial models are rare and usually restricted to one-dimensional (Bethe) integrable systems [154], and non-generic low dimensional systems, specifically designed to be solvable, such as the two dimensional Kitaev model [162]. The existence of DPT-II in non-integrable models is argued on the basis of perturbation techniques [160]. From this perspective, it is natural to ask, whether DPT-II can be investigated in (asymptotically) infinite dimensional systems, where many calculations become amenable to analytic considerations in an $1 / N$ expansion. Mean field models, such as the FC-TFIM

$$
\widehat{H}=-\frac{1}{2 N} \sum_{i, j=1}^{N} s_{i}^{z} s_{j}^{z}-\Gamma \sum_{i=1}^{N} s_{i}^{x}
$$

are typical candidates for these infinite dimensional systems. They are complementary to both, low-dimensional systems, as well as perturbative treatment of non-integrable systems. Besides the $1 / N$ expansion no additional expansion in coupling constants is necessary.

In the following section a heuristic semiclassical argument in favor for the existence of DPT-II in the FC-TFIM is given. This argument also indicates to expect non-analytic behavior in $r(w, t)$ for non-zero work density $w>0$, and the existence of DPT-II for quenches within the same equilibrium phase. This is in contrast to the one-dimensional counterpart where DPT-II only occur for quenches across the underlying equilibrium phase transition. Thereafter, in Sec. 4.3 , we give a more rigorous discussion based on a semiclassical expansion of the density of states and the overlap between the pre-quench ground state with post-quench energy eigenstates. These results confirm the heuristic expectation of Sec. 4.2 .

Though, the approaches of sections 4.2 and 4.3 seem equivalent in the sense that both rely on semiclassical techniques, their flavor is different. The former has a phase space flavor, while the latter has a spectral density flavor. Each provides a different perspective and complements the other.

\subsection{Phase space heuristics}

We want to mimic the work probability distribution (4.2) after a double quench by semiclassical quantities in the effective description ${ }^{2}$ given by

$$
H_{\mathrm{eff}}=-\frac{1}{2}\left(n_{+}-1 / 2\right)^{2}-\Gamma \sqrt{n_{+}-n_{+}^{2}} \cos (p),
$$

where $p$ is the conjugate momentum to the fraction of up spins $0<n_{+}=N_{+} / N<1$. To this end, we suggest the semiclassical analog $P_{\text {eff }}$ in Eq. (4.5) below. We do not derive a rigorous correspondence between the work distribution function (4.2) of the full quantum model and 4.5).

\footnotetext{
${ }^{2}$ The correspondence between (4.3) and (4.4) was elaborated in chapter 2 Recall that eigenvalues of $H_{\text {eff }}$ are

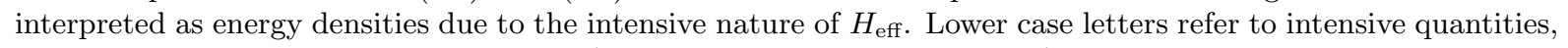
such as the fraction of up spins $n_{+}=N_{+} / N$ and the work density $w=W / N$.
} 


\section{Dynamical Phase Transitions}

But, we argue that it is plausible to expect $P(W, t) \rightarrow P_{\text {eff }}(W / N, t)$ in the thermodynamic limit $N \rightarrow \infty$. This is the reason why we only claim to give an heuristic approach to DPT-II in this section.

The double quench setup consists of preparing the system at time $t=0$ in the ground state $\left|\psi_{0}\right\rangle$ of the pre-quench Hamiltonian $H$ for some initial transverse field $\Gamma=\Gamma_{i}$. After driving the system with the post-quench Hamiltonian $H^{\prime}$ with $\Gamma=\Gamma_{f}$ for time $t$, the Hamiltonian is quenched back to $H$. The work probability distribution after the second quench is $P(W, t)=$ $\sum_{n} \delta\left(W-\left(E_{n}-E_{0}\right)\right)\left|\left\langle E_{n} \mid \psi_{0}(t)\right\rangle\right|^{2}$, cf. Eq. 4.2.

In the semiclassical limit $\hbar_{\text {eff }}=1 / N \rightarrow 0$ the ground state $\sum_{N_{+}} \psi_{0}\left(n_{+}\right)\left|N_{+}\right\rangle \in \mathcal{D}_{N}$ of $H$ is of large deviation form $\psi_{0}\left(n_{+}\right) \asymp \exp \left[-N f\left(n_{+}\right)\right]$. Let $\psi_{t}\left(n_{+}\right) \asymp \exp \left[-N f\left(n_{+}, t\right)\right]$ be the time evolution of $\psi_{0}\left(n_{+}\right)$with respect to the effective post-quench Hamiltonian. Since both, $\psi_{0}\left(n_{+}\right)$ and $\psi_{t}\left(n_{+}\right)$are of large deviation form, also the work distribution $P(W, t) \asymp \exp [-N r(W, t)]$ has large deviation form with rate function $r(W, t)$. We want to establish a relation between the two rate functions $f\left(n_{+}, t\right)$ and $r(W, t)$ in a Gaussian approximation. To do this, we employ the truncated Wigner approximation and the nearby orbit approximation in the classical phase space of the effective model.

Now we give a semiclassical definition of $P_{\text {eff }}$. Let $z_{0}$ be a point in phase space with energy $H_{\text {eff }}\left(z_{0}\right)$ according to the pre-quench Hamiltonian $H_{\text {eff. After evolving }} z_{0}$ according to Hamilton's equations $\dot{z}(t)=J \nabla H_{\text {eff }}^{\prime}(z)$ w.r.t. the post-quench Hamiltonian for time $t$, the particle is at $T_{t}^{\prime}\left(z_{0}\right)$. With respect to the pre-quench Hamiltonian $H_{\text {eff }}$, the particle has gained energy

$$
W_{t}\left(z_{0}\right)=H_{\mathrm{eff}}\left(T_{t}^{\prime} z_{0}\right)-H_{\mathrm{eff}}\left(z_{0}\right)
$$

This is the classical work performed by the double quench at the system.

Now, let $z_{0}$ be a random point in phase space, distributed according to the density $\mu_{0}$. The work(density) $w$ performed after the double quench then becomes random with probability $\mathbb{P}(w \in$ $\left.\left[W_{1}, W_{2}\right]\right)=\mu_{0}\left(W_{t}^{-1}\left(\left[W_{1}, W_{2}\right]\right)\right)$ where $\mu_{0}(A)=\int \mathbf{1}_{A} \mu_{0}(z) d z$. One finds the work probability density

$$
\begin{aligned}
P_{\mathrm{eff}}(w, t) d W & =\mathbb{P}(W \in[w, w+d W])=\mu_{0}\left(W_{t}^{-1}([w, w+d W])\right) \\
& =\sum_{z^{*} \in W_{t}^{-1}(w)} \mu_{0}\left(z^{*}\right)\left\|\nabla W_{t}\left(z^{*}\right)\right\|^{-1} d W \\
& =\left[\int \mu_{0}(z) \delta\left(W_{t}(z)-w\right) d z\right] d W .
\end{aligned}
$$

This is the classical analog of 4.2.

We give a non-rigorous argument indicating how $P$ converges to $P_{\text {eff }}$ in the semiclassical limit. Eq. (4.2) is written as $P(W, t)=\operatorname{Tr}\left[\delta\left(\widehat{H}-E_{0}-W\right)|\psi(t)\rangle\langle\psi(t)|\right]$. From now on, we assume that the pre-quench eigenstates $E_{n}$ are non-degenerate, such that $P(W, t)=\operatorname{Tr}\left[\left|E_{n}\right\rangle\left\langle E_{n} \mid \psi_{t}\right\rangle\left\langle\psi_{t}\right|\right]$, where $W=\left(E_{n}-E_{0}\right)$, and $\left|\psi_{t}\right\rangle$ is the time evolved initial state $\left|\psi_{0}\right\rangle=\left|E_{0}\right\rangle$ w.r.t. the post quench Hamiltonian. Rewriting $\operatorname{Tr} \widehat{A} \widehat{B}=\int W_{\widehat{A}} W_{\widehat{B}}$ in terms of the Wigner transforms $W_{\widehat{A}}, W_{\widehat{B}}$ of $\widehat{A}, \widehat{B}$, yields,

$$
P(W, t)=\int d z W_{\left|E_{n}\right\rangle\left\langle E_{n}\right|}(z) W_{\left|\psi_{t}\right\rangle\left\langle\psi_{t}\right|}(z) .
$$


This is exact. Now, we employ three approximations to obtain

$$
\begin{aligned}
P(W, t) & =\int d z W_{\left|E_{n}\right\rangle\left\langle E_{n}\right|}(z) W_{\left|\psi_{t}\right\rangle\left\langle\psi_{t}\right|}(z) \\
& \approx \int d z \delta\left[H_{\mathrm{eff}}(z)-E_{n} / N\right] \mu_{t}(z) \\
& \approx \int d z \delta\left[H_{\mathrm{eff}}(z)-E_{n} / N\right] \mu_{0}\left(T_{-t}^{\prime} z\right) \\
& =\int d z \delta\left[H_{\mathrm{eff}}\left(T_{t}^{\prime} z\right)-E_{n} / N\right] \mu_{0}(z) \\
& \approx \int d z \delta\left[H_{\mathrm{eff}}\left(T_{t}^{\prime} z\right)-H_{\mathrm{eff}}(z)-W / N\right] \mu_{0}(z)=P_{\mathrm{eff}}(W / N, t) .
\end{aligned}
$$

The approximations in the above order consist of, first, replacing the spectral Wigner function $W_{\left|E_{n}\right\rangle\left\langle E_{n}\right|}$ by a uniformly distributed probability density on the invariant torus associated to the energy $E_{n} / N$ 94, 96]. Second, the time evolved Wigner function $W_{|\psi(t)\rangle\langle\psi(t)|}=: \mu_{t}$ is approximated by the initial Wigner function $\mu_{0}$ evaluated at the characteristics of the classical Hamiltonian flow $T_{t}^{\prime}$, i.e. $\mu_{t}(z) \approx \mu_{0}\left(T_{-t}^{\prime} z\right)$. This approximation amounts to replacing Moyal's Eq. for the Wigner function by the classical Liouville's Eq. and goes under the name of the truncated Wigner approximation (TWA) [88]. It is known [88] that the TWA gives the leading term of the full quantum dynamics in a semiclassical limit and the leading order correction to it is suppressed as $1 / N^{2}$. The change of variables $z \mapsto T_{-t}^{\prime}(z)$ in the line below has unit Jacobi determinant, because it is symplectic. Third, as the integration is against the spectral Wigner function $\mu_{0}$ of the prequench ground state, most points are sampled form the energy shell where $H_{\text {eff }}(z) \approx E_{0}$. This justifies the last approximation.

Remark. The fact that the $n$th spectral Wigner function $W_{n}(z) \propto \delta\left(H_{\text {eff }}(z)-E_{n}\right)$ is uniformly distributed on the associated ${ }^{3}$ energy shell in the classical limit follows from the WKB ansatz $\psi_{n}(x) \sim \exp \left(i S(x) / \hbar_{\mathrm{eff}}\right)$, where $S(x)=\int_{x_{0}}^{x} p_{n}\left(x^{\prime}\right) d x^{\prime}$ is Maupertuis's action, and $H\left(p_{n}(x), x\right)=E_{n}$, cf. Sec. 2.3. Approximating the exponent in $W_{n}(z)=\int d \eta \psi_{n}(x-\eta / 2) \bar{\psi}_{n}(x+\eta / 2) e^{i p \eta / \hbar_{\mathrm{eff}}}$ to first order in $\eta$, yields $W_{n} \propto \delta\left(H_{\text {eff }}-E_{n}\right)$. In a more careful semiclassical limit 96,127$]$ the exponent is expanded to second order in $\eta$ and the integration is solved by the (uniform generalization 125] of the) saddle-point approximation. As a consequence, the delta function is softened and $W_{n}$ has also support outside and inside the torus (Ref. [96] calls it a fringed torus). This more careful approximation resolves more structure of the wave function, such as oscillations on a scale of $\hbar_{\mathrm{eff}}^{2 / 3}$, but is not strictly necessary for the investigation of DPT-II. The reason is that non-analytic behavior can only occur in the limit $N \rightarrow \infty$, and hence the leading order approximation in $\hbar_{\mathrm{eff}}=1 / N$ suffices to establish the existence and non-existence of DPT.

Non analyticities from the effective phase space point of view. Based on 4.5 we investigate the behavior of the work probability rate function $r_{\text {eff }}$ of $P_{\text {eff }}(w, t) \asymp \exp \left[-N r_{\text {eff }}(w, t)\right]$ as a function of time at zero work density $w=0$, i.e. we are interested in the limit

$$
r_{\mathrm{eff}}(t)=-\lim _{N \rightarrow \infty} \frac{1}{N} \log P_{\mathrm{eff}}(w=0, t)=-\lim _{N \rightarrow \infty} \frac{1}{N} \log \int \mu_{0}(z) \delta\left(W_{t}(z)\right) .
$$

We further assume, that the density $\mu_{0}(z)=\mathcal{N} \exp [-N r(z)]$ is of large deviation form (not necessarily Gaussian) with rate function $r(z)$ (we denote this rate function by the same letter as the rate

\footnotetext{
${ }^{3}$ This association is due to the Bohr-Sommerfeld quantization condition $120 \frac{1}{2 \pi} \oint_{H(z)=E_{n}} p(x) \cdot d x=(n+1 / 2) \hbar_{\mathrm{eff}}$, where the integration is over the $E_{n}$ energy shell and $n \in \mathbb{N}_{0}$.
} 


\section{Dynamical Phase Transitions}

function of the work probability distribution). The integral can be computed within the saddle point approximation as

$$
\int \mu(z) \delta\left(W_{t}(z)\right)=\mathcal{N} \sum_{z^{*}} \exp \left[-N r\left(z^{*}\right)\right]\left(\frac{2 \pi}{N}\right)^{1 / 2} \sqrt{\frac{\operatorname{det} r^{\prime \prime-1}}{\left|W_{t}^{\prime} r^{\prime \prime-1} W_{t}^{\prime}\right|}}[1+\mathcal{O}(1 / N)],
$$

where the summation is over all $\arg \min z^{*}$ of $r(z)$ subject to the constraint $W_{t}(z)=0, r^{\prime \prime}-1$ denotes the inverse Hessian of $r(z)$ at $z=z^{*}$, and $W_{t}^{\prime}$ is the gradient of $W_{t}$ at $z=z^{*}$. We have used the expansion $W_{t}(z) \approx W_{t}\left(z^{*}\right)+W_{t}^{\prime}\left(z^{*}\right)\left(z-z^{*}\right)+\frac{1}{2}\left(z-z^{*}\right) W_{t}^{\prime \prime}\left(z^{*}\right)\left(z-z^{*}\right)$ and $r(z) \approx$ $r\left(z^{*}\right)+r^{\prime}\left(z^{*}\right)\left(z-z^{*}\right)+\frac{1}{2}\left(z-z^{*}\right) r^{\prime \prime}\left(z^{*}\right)\left(z-z^{*}\right)$ around $z=z^{*}$. We can now compute $r_{\text {eff }}$ in the limit $N \rightarrow \infty$ by noting that $\left(\sum_{n} x_{n}^{N}\right)^{1 / N} \rightarrow \max \left|x_{n}\right|$,

$$
r_{\text {eff }}(t)=\min _{z^{*} \in\left\{z: W_{t}(z)=0\right\}}\left|r\left(z^{*}\right)\right| .
$$

The time dependence is implicitly induced by the time dependence of the constraint set $\{z$ : $\left.W_{t}(z)=0\right\}$. The fact that the min-function is non-differentiable where two of its arguments are identical is the reason why $r_{\text {eff }}(0)$ can have non-differentiable points (e.g. kinks) in time, see Fig. 4.2. We stress that this argument only yields candidates for critical times $t^{*}$, at which DPT-II can occur. The argument is not sufficient. That is, even though the $\arg \min$ of $|r(z)|$ may change discontinuously at $t=t^{*}$, the rate function $r_{\text {eff }}(t)$ can still be analytic. In fact, we illustrate this explicitly in an harmonic oscillator model below.

Remark (on the nested structure of the constraint set $W_{t}(z)=0$ ). As time proceeds the constraint set $\left\{z: W_{t}(z)=0\right\}$ develops ever more complicated and detailed structures. This seems to be the rule, rather than an exception and also happens for simple integrable flows. At $t=0$ all points $z$ in phase space obey $W_{t}(z)=H_{\mathrm{eff}}\left(T_{t}^{\prime} z\right)-H_{\mathrm{eff}}(z)=0$. Fixed points of the post-quench Hamiltonian flow $T_{t}^{\prime}$ obey $W_{t}(z)=0$ for all times. At those points different branches of the constraint set start off and extend to complex structures in phase space. The constraint set at a later time $t_{2}$ is not simply the time evolved constraint set of a former time $t_{1}$ by the difference $\Delta=\left(t_{1}-t_{2}\right)$. This is because $T_{\Delta}^{\prime} \delta\left[H_{\mathrm{eff}}\left(T_{t_{1}}^{\prime} z\right)-H_{\mathrm{eff}}(z)\right]=\delta\left[H_{\mathrm{eff}}\left(T_{t_{2}}^{\prime} z\right)-H_{\mathrm{eff}}\left(T_{-\Delta}^{\prime} z\right)\right] \neq \delta\left[H_{\mathrm{eff}}\left(T_{t_{2}}^{\prime} z\right)-H_{\mathrm{eff}}(z)\right]$. In 127,169 the time evolution of one-dimensional submanifolds in phase space under Hamilton's equations of motion is investigated in the context of semiclassical analysis of quantum maps. In spite of the fact that this mechanism is different to the evolution of the constraint set, the emerging structures show similarities, and the fixed points of the flow play a similarly important role.

Remark (on non-zero work). The argument leading to (4.6) also applies to the situation of nonvanishing work density $w \neq 0$. In this case the rate function $r_{\text {eff }}(w, t)=\min _{z^{*}}\left|r\left(z^{*}\right)\right|$ is the minimum over the minima $z^{*}$ of $\left\{z: W_{t}(z)-w=0\right\}$. In view of this semiclassical picture, one expects non-analytic behavior also for $r_{\text {eff }}(w, t)$ and $w \neq 0$. Indeed, numerical results based on ED indicate non-analytic behavior also for non-vanishing work density. This is in contrast to the 1D-TFIM, where the non-zero work probability rate function is analytic for all times [59]. 


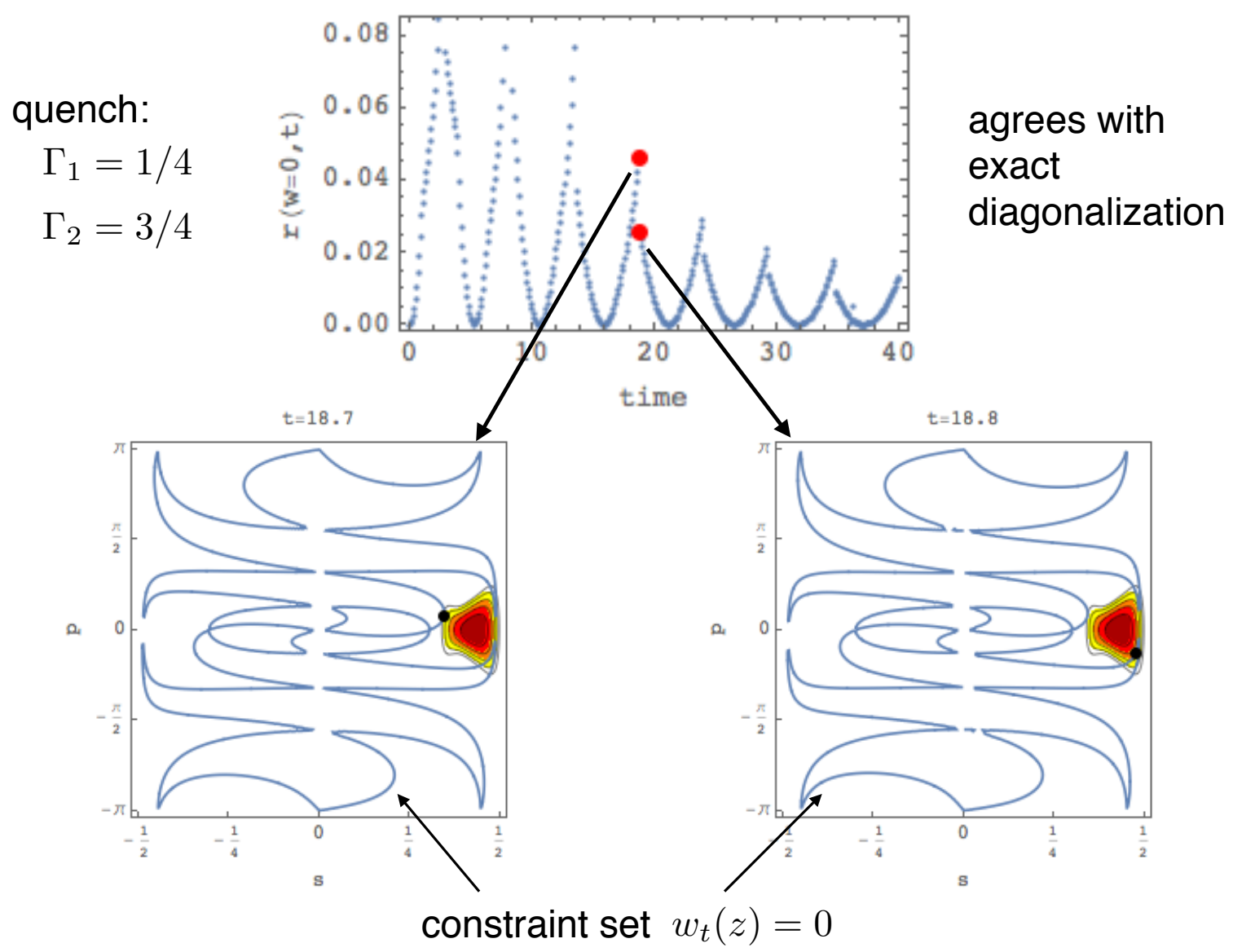

Figure 4.2: DPT-II in a semiclassical phase space picture for the quench $\Gamma_{1} \mapsto \Gamma_{2}$. Non-analytic behavior of $r_{\text {eff }}(t)$, cf. (4.6), occurs at critical times (top). At a critical time the phase space coordinates of the global minimum of $\left\{|r(z)|: W_{t}(z)=0\right\}$ changes. This is illustrated in the bottom row by showing the position of the global minimum (black dot) in phase space just before (left) and after (right) a critical time. The minimization is over the constraint set $\left\{z: W_{t}(z)=0\right\}$ (blue line). The heatmap indicates the level sets of the Wigner function $\mu_{0}(z) \asymp \exp [-N r(z)]$, equivalently the level sets of its rate function $r(z)$. 


\subsection{Semiclassical overlaps}

The preceding section explained how one can understand the origin of the non-analyticities of the work distribution rate function in a semiclassical picture. The key observation is that the non-analytic behavior originates from maximizing the Wigner function on the constraint set $\{z$ : $\left.W_{t}(z)=0\right\}$. For late times the constraint set develops a complicated 'nested' structure, see Fig. 4.2. This makes the prediction of the non-analyticities difficult. In this section we go back to the full quantum mechanical spin picture to complement the understanding of the origin of the non-analyticities. Thereby, we employ semiclassical results about the spectral density of states and the ground state. The following analysis is only applied to the Loschmidt echo, i.e. zero performed work.

The starting point is the expansion of the Loschmidt echo

$$
P(W=0, t)=\left.\left.\left|\sum_{n=0}^{N}\right|\left\langle E_{n} \mid \psi_{0}\right\rangle\right|^{2} e^{-i E_{n} t}\right|^{2}
$$

in the post-quench eigenstates $\left|E_{n}\right\rangle$ of $(4.3)$ on the $(N+1)$ dimensional Dicke space $\mathcal{D}_{N}$. After appropriate scaling, the energy levels $E_{n}$ and the overlaps $\left|\left\langle E_{n} \mid \psi_{0}\right\rangle\right|^{2}$ as a function of $n$ collapse on a curve in the semiclassical limit $N \rightarrow \infty$. We denote these functions by

$$
\begin{aligned}
E_{n} / N & \rightarrow \epsilon(n / N), \\
\left|\left\langle E_{n} \mid \psi_{0}\right\rangle\right|^{2} & \rightarrow \chi(n / N) \asymp e^{-N f(n / N),},
\end{aligned}
$$

and refer to them as energy density $\epsilon$ and overlaps $\chi$ as a function of the index function $x=n / N$, respectively. Since the indices $n$ take values in $\mathbb{Z}_{N+1}=\{0,1, \cdots N\}$, the index function $x$ samples the unit interval equidistantly with spacing $1 / N$. In the large $N$ limit it is fair to approximate $x$ as a continuous variable on the unit interval, and view $\epsilon:[0,1] \rightarrow \mathbb{R}$ and $f:[0,1] \rightarrow \mathbb{R}$ in (4.7) as $N$-independent functions on the unit interval. We remark that the following discussion applies not only to the FC-TFIM (4.3), but to any system on a $(N+1)$ dimensional Hilbert space with the large $N$ property 4.7 ).

In this notation the large $N$ limit of $P(W=0, t)$ reads

$$
P(W=0, t)=\left|\sum_{x \in \mathbb{Z}_{N+1} / N} \chi(x) e^{-i N \epsilon(x) t}\right|^{2} \asymp\left|\sum_{x \in \mathbb{Z}_{N+1} / N} e^{-N f(x)} e^{-i N \epsilon(x) t}\right|^{2}
$$

We want to evaluate this summation to first order in the exponent, in order to get the rate function of $P(W=0, t)$ as a function of $t$. The fact that $\chi$ is of large deviation form implies that only a few terms in the sum give significant contributions, namely only those terms for which $x$ is close to the $\arg \min x^{*}$ of $f(x)$. However, since we are interested in the full rate function of $P(W=0, t)$, which depends on the tail of unlikely events in $\chi$, a careful treatment of the summation is necessary. In particular, as shown below, it is not sufficient to sum the most significant $\sqrt{N}$ terms in a saddle point fashion directly. A more careful summation technique is provided by Poisson's summation

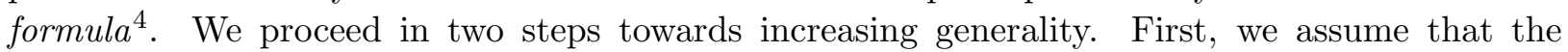
energy densities $\epsilon\left(\mathbb{Z}_{N+1} / N\right)$ are equidistantly spaced. The main result in this situation is given by Eq. (4.13). Second, we allow for non-vanishing curvature $\epsilon^{\prime \prime} \neq 0$. The latter is a generalization

\footnotetext{
${ }^{4}$ The Poisson summation formula has a longstanding tradition. It has e.g. been used in the computation of the Gauss sum [85], and is the basis of the fundamental transformation of the Jacobi theta function. In fact, the summation (4.8) is closely related to the Jacobi theta function $\theta_{3}$ if $f$ is a quadratic function and $\epsilon$ is linear.
} 
of the former and culminates in Eq. 4.22). The former result is obtained from the latter in the special case of vanishing curvature. We hope that this redundancy in the presentation yields more clarity.

\subsubsection{Equidistant spectrum}

We assume that the post-quench spectrum is equidistant, equivalently, that the energy density $\epsilon(x)=e_{0}+\omega x$ in $4.7 \mathrm{a}$ is linear as a function of the index. The proportionality factor $\omega=1 / \rho$ is the inverse density of states. Equation 4.8 then becomes

$$
P(W=0, t) \asymp\left|\sum_{x \in \mathbb{Z} / N} \chi(x) e^{-i N \omega x t}\right|^{2},
$$

where the summation is extended to the doubly infinite sum over $\mathbb{Z} / N$ in view of the exponential decay of $\chi \asymp e^{-N f(x)}$. This is valid as long as the roots of $f(x)$ are not at the boundary $x=0$ and $x=1$. By Poisson's summation formula $5^{5}$ we may rewrite the discrete Fourier series (4.9) as the summation

$$
P(W=0, t) \asymp\left|\sum_{\xi \in 2 \pi N \mathbb{Z}} \widehat{\chi}(N \omega t+\xi)\right|^{2},
$$

over the Fourier transform $\widehat{\chi}(p)=\int_{\mathbb{R}} d x \chi(x) e^{-i p x}$. Due to the uncertainty principle, the function $\chi$ and its Fourier transform $\hat{\chi}$ cannot simultaneously have a large deviation form. However, by appropriate rescaling of the argument of $\widehat{\chi}$, it follows from Laplace's principle that $\widehat{\chi}(N p)$ is of large deviation as a function of $p$. More precisely, the rate function $g$ of $\widehat{\chi}(N p) \asymp e^{-N g(p)}$ is related to the rate function $f$ of $\chi(x) \asymp e^{-N f(x)}$ by

$$
g(p)=-f^{*}(i p)
$$

where $f^{*}(k)=\sup _{x}(k x-f(k))$ denotes the Legendre-Fenchel transformation of $f$. This is due to Laplace's principle, and can also be viewed as a consequence of the Wick-rotated Gärtner-Ellis theorem [172,173. Using this result in 4.10 gives

$$
P(W=0, t) \asymp\left|\sum_{\xi \in 2 \pi \mathbb{Z}} e^{-N g(\omega t+\xi)}\right|^{2} .
$$

And thus the rate function $r(t)=-\lim _{N \rightarrow \infty} \frac{1}{N} \log P(W=0, t)$ is

$$
r(t)=2 \min _{\xi \in 2 \pi \mathbb{Z}} \Re g(\omega t+\xi)=2 \min _{n \in \mathbb{Z}} \Re g(\omega(t+n T)),
$$

\footnotetext{
${ }^{5}$ Let the Fourier transform of $f \in L^{1}\left(\mathbb{R}^{d}\right)$ be $\widehat{f}(p)=\int d^{d} x f(x) e^{-i x \cdot p}$, and $\Gamma=M\left(\mathbb{Z}^{d}\right)$ be a subgroup of $\mathbb{R}^{d}(M$ being a full rank $d$ by $d$ matrix). Then, the Poisson summation formula reads 170

$$
\sum_{x \in \Gamma} f(x) e^{-i p x}=\frac{1}{|\operatorname{det} M|} \sum_{\xi \in \Gamma^{*}} \widehat{f}(p+\xi),
$$

where the dual lattice $\Gamma^{*}=\left\{p \in \mathbb{R}^{d}: e^{i p \cdot x}=1, \forall x \in \Gamma\right\}$ is the orthogonal complement of $\Gamma$ in the Pontryagin dual. In words, restricting $f(x)$ to the subgroup $x \in \Gamma$ (left hand side) translates under Fourier transform to averaging $\widehat{f}(p)$ over $\Gamma^{*}$ (right hand side) 171 . In particular, for $d=1, \Gamma=\mathbb{Z}$ one has $\Gamma^{*}=2 \pi \mathbb{Z}$ and the classical Poisson summation formula

$$
\sum_{x \in \mathbb{Z}} f(x) e^{-i p x}=\sum_{\xi \in 2 \pi \mathbb{Z}} \widehat{f}(p+\xi) .
$$
}




\section{Dynamical Phase Transitions}

where $T=2 \pi / \omega$.

Equation 4.13 is the final result of this section. It is the rate function of $P(W=0, t)$ for a system obeying (4.7), and with constant post-quench spectral density $\rho=1 / \omega$, i.e. linear $\epsilon$. Moreover, it is assumed that the initial state has significant contributions in the bulk of the postquench spectrum, i.e. that the minima of the rate function $f(x)$ are not at the boundary $x \in\{0,1\}$. Prior to discussing specific examples for $f$, we infer some generic properties.

- Without loss of generality $\square^{6} \Re(p)$ has a global minimum and root at $p=0$, and $\Re g$ is convex around $p=0$ (as a consequence of $f^{*}$ being convex).

- Because the post-quench spectral density $\rho=1 / \omega$ is constant, any quantity derived from the time evolved state $\sum c_{n} e^{-i E_{n} t}\left|E_{n}\right\rangle$ must be periodic with period $T=2 \pi / \omega$. Consequently, as $\Re g(\omega t)$ vanishes at $t=0$ (see footnote 6), it vanishes at all integer multiples of $T=2 \pi / \omega$. This observation is confirmed by 4.13). At $t=n T$ for some integer $n$, the minimum in (4.13) is obtained for $\xi=-2 \pi n$. Between two consecutive roots of $r(t)$, say between $t_{1}=n T$ and $t_{2}=(n+1) T$ the arg min changes from $\xi_{1}=-2 \pi n$ to $\xi_{2}=-2 \pi(n+1)$ at the point where the two functions $2 \Re g\left(\omega t+\xi_{1}\right)$ and $2 \Re g\left(\omega t+\xi_{2}\right)$ intersect. Let us refer to this intersection as $t_{n}^{*}$, cf. Fig. 4.3 .

- If $\Re g$ is analytic, $t=t_{n}^{*}$ are the only instants of time at which $r(t)$ may potentially be non-analytic. Hence $\left\{t_{n}^{*}: n \in \mathbb{Z}\right\}$ are candidates for DPT-II. To sufficiently conclude the existence of a DPT-II at $t=t_{m}^{*}$, one must investigate the analytic properties of the piecewise defined function

$$
\begin{cases}2 \Re g(\omega t-2 \pi n), & t \leq t_{n}^{*} \\ 2 \Re g(\omega t-2 \pi(n+1)), & t \geq t_{n}^{*}\end{cases}
$$

at $t=t_{n}^{*}$, cf. Fig. 4.3 . Below we are stating two explicit examples that demonstrate the existence (when $\chi$ is Gaussian) and absence (for quench in harmonic oscillator) of DPT-II at $t_{n}^{*}$, respectively.

- Mathematically, the minimization over the $N$-independent translations of $\Re g$ in $(4.13)$ is a consequence of the Poisson summation formula and the large deviation nature of the summands. The physical reason, why the Poisson summation formula is applicable is the fact that the energy levels $E_{n}$ are discrete and the gap between consecutive levels (in the bulk) scales independently of system size, i.e. $\left(E_{n+1}-E_{n}\right)=\mathcal{O}\left(N^{0}\right)$. The latter property is rather unusual and has to do with the highly symmetric situation of the fully connected lattice in the low dimensional Dicke space. As the energy range is extensive, i.e. linear in $N$, and the Dicke space dimension is $(N+1)$, the average gap between consecutive energies is independent of $N$. In contrast, a more common situation is that of an extensive spectral range, and a Hilbert space dimension that scales exponential with the system size. In such systems the typical gap between consecutive energies is exponentially small in system size.

Example (Symmetric $\Re g$ ). A wide class of examples is the situation when $\Re g$ is symmetric around $p=0$, and convex. Then, the unique intersection between two consecutive branches $\Re g(\omega t+2 \pi n)$ and $\Re g(\omega t+2 \pi(n+1))$ occurs at $t_{n}^{*}=(n+1 / 2) T$ and the minimization in 4.13) yields

$$
r(t)=2 \Re g\left([\omega t]_{[-\pi, \pi]}\right),
$$

where []$_{[a, b]}: \mathbb{R} \rightarrow[a, b]$ denotes the projection onto the interval $[a, b]$. More explicitly, $[t]_{[-a, a]}=$ $t-2 a\left\lfloor\frac{t}{2 a}+\frac{1}{2}\right\rfloor$, where $\lfloor x\rfloor$ denotes the largest integer below $x$.

\footnotetext{
${ }^{6}$ This follows from the invariance of 4.9 under translation of the summation index. Physically, it is the trivial
} observation that the probability to find the system in the initial state $\left|\psi_{0}\right\rangle$ at time $t=0$ is unity. 

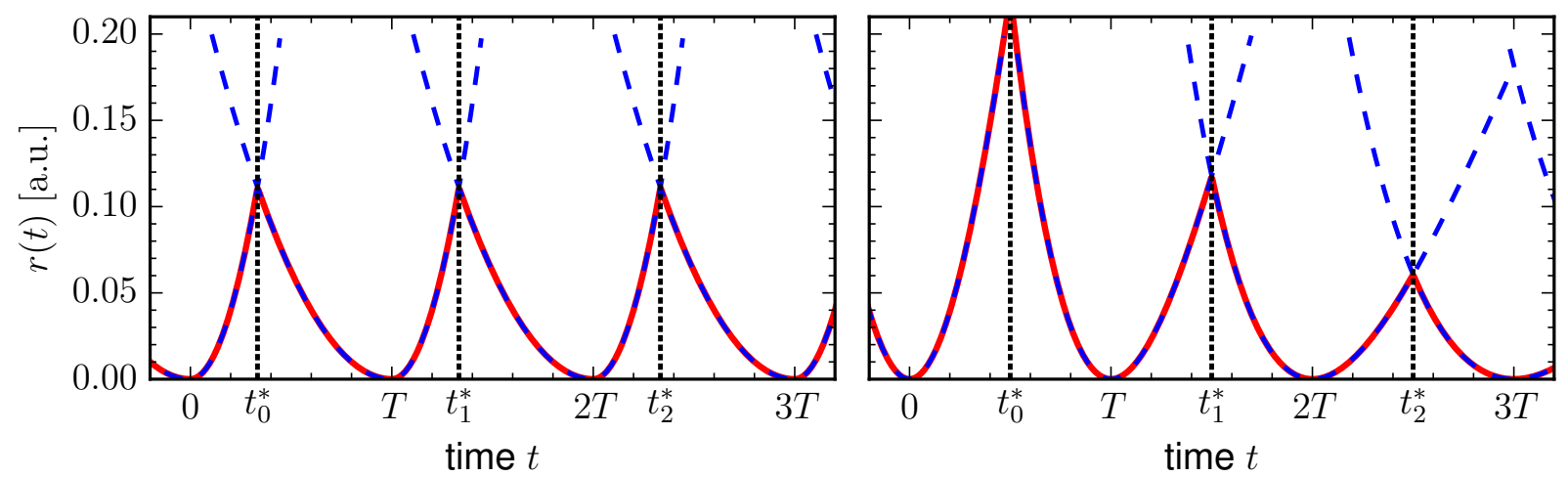

Figure 4.3: Schematic sketch of branch crossings leading to DPT-II for constant post-quench spectral density (left), and for non-constant spectral density (right). The rate function $r(t)$ (solid red line) of the work probability distribution $P(W=0, t)=\left|\left\langle\psi_{0} \mid \psi_{t}\right\rangle\right|^{2}$, i.e. the Loschmidt echo, is the point-wise minimum over a family of rate functions (dashed blue line) according to Eqs. (4.13) (left) and (4.22) (right). The members of the family are obtained by translating $\Re g$, cf. (4.11), by integer multiples of $T=2 \pi / \omega$ as a consequence of Poisson's summation formula. Vertical black dotted lines indicate a crossing between two members of the family at $t=t_{n}^{*}$. These are candidates for DPT-II at which $r(t)$ can be non-analytic as a function of time. For non-constant density of states (right) a dephasing effect leads to a inversely quadratic decay of the envelope of $r(t)$ with time. Roots of $r(t)$ occur at integer multiples of $T$ when (i) the wave function has a perfect recurrence (in the case when the post-quench spectrum is equidistant, left), and (ii) the wave function has most overlap with the pre-quench ground state (when the post-quench spectrum is non-equidistant, right). 


\section{Dynamical Phase Transitions}

Example (Gaussian overlaps and Jacobi's theta function, cf. Fig. 4.4). If the overlap function $\chi$ is Gaussian distributed with variance $C$, i.e. for $f(x)=\frac{1}{2} x^{2} / C$, the Legendre transform is $f^{*}(k)=\frac{1}{2} C k^{2}$, such that $g(p)=\frac{1}{2} C p^{2}$ and (4.14) entails

$$
r(t)=C \omega^{2}[t]_{[-T / 2, T / 2]}^{2},
$$

where $T=2 \pi / \omega$. The discontinuity of the projection at the boundary points $(\mathbb{Z}+1 / 2) T$ entails kinks of $r(t)$ at $t_{n}^{*}=(n+1 / 2) T$, cf. Fig. 4.4 .

If $f$ is quadratic the series in Eq. 4.10 is Jacobi's theta function by $\theta_{3}$. In this case the Poisson transformation between (4.10) and (4.11) is known as the fundamental transformation of $\theta_{3}$ [174].

The following example shows that the changing of a branch in the minimization of 4.13 is not sufficient for the existence of a DPT-II.

Example (Quench in harmonic oscillator, cf. Fig. 4.4). We discuss the existence of DPT-II for a double quench in a purely harmonic one-dimensional effective Hamiltonian (as in (4.4) we have $\left.\hbar_{\text {eff }}=1 / N\right)$. More precisely, let the pre-quench Hamiltonian be $H_{\Delta}=\left[(q-\Delta)^{2}+p^{2}\right] / 2$ and the post-quench Hamiltonian be $H=\left(q^{2}+p^{2}\right) / 2$, i.e. the Hamiltonians differ by a relative shift $\Delta$ in position. The post-quench eigenstates are given by $\left\langle q \mid e_{n}\right\rangle=(N / \pi)^{1 / 4}\left(2^{n} n !\right)^{-1 / 2} H_{n}(\sqrt{N} q) e^{-N q^{2} / 2}$ with corresponding energy density

$$
e_{n}=(n+1 / 2) / N \text {, }
$$

where $H_{n}$ denotes the $n$th Hermite polynomials. We use a lower case ' $e$ ' in (4.16) to emphasize that the harmonic oscillator Hamiltonian measures energy density. The initial state $\psi_{0}(q)=$ $\left\langle q-\Delta \mid e_{0}\right\rangle=(N / \pi)^{1 / 4} e^{-N(q-\Delta)^{2} / 2}$ is the ground state of the pre-quench Hamiltonian. Using the generating function of the Hermite polynomials, $e^{2 \sqrt{N} q t-t^{2}}=\sum_{n} H_{n}(\sqrt{N} q) t^{n} / n$ !, one obtains the overlap: 7

$$
\begin{aligned}
\left\langle\psi_{0} \mid e_{n}\right\rangle & =(N / \pi)^{1 / 2}\left(2^{n} n !\right)^{-1 / 2} \partial_{t}^{n} \int d q e^{-N(q-\Delta)^{2} / 2} e^{2 \sqrt{N} q t-t^{2}} e^{-N q^{2} / 2} \\
& =(N / \pi)^{1 / 2}\left(2^{n} n !\right)^{-1 / 2} \partial_{t}^{n}(N / \pi)^{1 / 2} e^{-N \Delta^{2} / 4+\sqrt{N} \Delta t} \\
& =e^{-N \Delta^{2} / 4}(\sqrt{N} \Delta)^{n} / \sqrt{2^{n} n !}
\end{aligned}
$$

Hence, the probabilities $\left|\left\langle\psi_{0} \mid e_{n}\right\rangle\right|^{2}=e^{-\lambda} \lambda^{n} / n ! \equiv P_{\lambda}(n)$ are Poisson distributed with expectation value $\lambda=N \Delta^{2} / 2$ and variance $\lambda$. Therefore, $P(w=0, t)=\left|\sum_{n} P_{\lambda}(n) e^{-i t n}\right|^{2}=e^{-2 \lambda}\left|\exp \left(\lambda e^{-i t}\right)\right|^{2}=$ $e^{-2 \lambda+2 \lambda \cos t}$, which yields the rate function

$$
r(w=0, t)=\Delta^{2}(1-\cos t) .
$$

This is an exact result, and shows that the rate function of $P(W=0, t)$ is analytic for all times $t$.

We want to rederive (4.18) from the point of view facilitated by 4.13 . As a function of $x=n / N$ the overlaps are of large deviation form $\left|\left\langle\psi_{0} \mid e_{n}\right\rangle\right|^{2} \asymp e^{-N f(x)}$ with rate function

$$
f(x)=x\left(\log \frac{2 x}{\Delta^{2}}-1\right)+\frac{\Delta^{2}}{2}, \quad x>0,
$$

(this follows from using Stirling's approximation in the Poisson distribution $P_{\lambda}(n)$ ), and the energy density $\epsilon(x)=x+1 /(2 N)$ is linear with $\omega=\epsilon^{\prime}=1$, cf. (4.16). In other words, the exact Eqs. (4.16) and (4.17) are of the form required by (4.7). Evaluating the Legendre-Fenchel transformation

\footnotetext{
${ }^{7}$ Interestingly, the quadratic term $t^{2}$ in the exponent cancels after completing the square in the integration variable.
} 
$f^{*}(k)=\left(e^{k}-1\right) \Delta^{2} / 2$ of 4.19 at $k=i \omega t$ gives $g(\omega t)=\left(1-e^{i \omega t}\right) \Delta^{2} / 2$ according to 4.11). As $\Re g$ is analytic and $2 \pi$-periodic, the minimization in (4.13) is over a single function, and hence, $r(t)=\Delta^{2}(1-\cos t)$ in agreement with 4.18.

This example demonstrates that the existence of DPT-II depends on the details in the exponential tails of the overlap function $\chi \asymp e^{-N f}$. If one expands the rate function $f$ to second order around its minimum at $x=\Delta^{2} / 2$, cf. (4.19) (this amounts to approximating the Poisson distribution $P_{\lambda}$ for large $\lambda$ by a Gaussian with variance $C=\lambda$ ), and thereby change the tails of $\chi$, then (4.14) erroneously predicts non-analytic behavior of $r(t)$ at odd integer multiples of $T$, cf. Eq. 4.15 and Fig. 4.4 .

There is another way to obtain the result 4.18 by working directly in phase space. The Wigner function of the $n$th eigenfunction $\left|e_{n}\right\rangle$ of the harmonic oscillator is given by $W_{n}(q, p)=$ $(-1)^{n} \frac{N}{\pi} L_{n}(4 N u) e^{-2 N u}$, where $L_{n}$ denotes the Laguerre Polynomials and $u=\left(q^{2}+p^{2}\right) / 2[175]$. In particular, the Wigner function of the pre-quench ground state is $\mu_{0}(q, p)=W_{0}(q-\Delta, p)=$ $\frac{N}{\pi} e^{-N\left((q-\Delta)^{2}+p^{2}\right)}$. Due to the Ehrenfest theorem the quantum mechanical time evolution of an initial Wigner function in a harmonic potential agrees with the classical Liouville dynamics. Therefore, $\mu_{t}(z)=\mu_{0}\left(T_{-t} z\right)$ where $T_{t}(z)=S_{t} z=\left(\begin{array}{cc}\cos t & \sin t \\ -\sin t & \cos t\end{array}\right) z$ is the linear symplectic flow induced by the post-quench Hamiltonian, and the computation of $P(w=0, t)$ reduces to a Gaussian integration

$$
\begin{aligned}
P(w=0, t) & =\left|\left\langle\psi_{0} \mid \psi_{t}\right\rangle\right|^{2}=\frac{1}{2 \pi} \int_{\mathbb{R}^{2}} \mu_{0}(z) \mu_{t}(z) d z \\
& \propto \int e^{-N\left(\|z-(\Delta, 0)\|^{2}+\left\|S_{-t} z-(\Delta, 0)\right\|^{2}\right)} d z \\
& \propto e^{-N \Delta^{2}(1-\cos t)},
\end{aligned}
$$

confirming Eq. 4.18.

\subsubsection{Non-equidistant spectrum}

Now, we discuss the situation when the post quench spectrum is not equidistant, equivalently, when the density of states $\rho(e)=\left.\epsilon^{\prime}(x)\right|_{x=\epsilon^{-1}(e)}$ is not constant.

We assume that the rate function $f(x)$ in $(4.7 \mathrm{~b}$ ) has unimodal real part $\Re f$ and attains a unique minimum at $x=x^{*}$. As in the previous Sec. we assume that the energy density $e=\epsilon\left(x^{*}\right)$ at this index is in the bulk of the post-quench Hamiltonian, i.e. $0<x^{*}<1$. Using the expansion $\epsilon(x)=e+\omega\left(x-x^{*}\right)+\Omega\left(x-x^{*}\right)^{2} / 2+\mathcal{O}\left(x-x^{*}\right)^{3}$ of the energy density in 4.7a yields

$$
P(W=0, t) \asymp\left|\sum_{x \in \mathbb{Z} / N} \chi(x) \alpha(x) e^{-i N \omega x t}\right|^{2}, \quad \text { where } \quad \alpha(x)=e^{-i N \Omega t x^{2} / 2}
$$

(after shifting the summation index $x \mapsto x+x^{*}$, and denoting the translated overlaps by the same symbol $\chi)$. The parameters $\omega=1 / \rho(e)$ and $\Omega=-\rho^{\prime}(e) / \rho(e)^{3}$ can be related to the density of states $\rho$ and its derivative $\rho^{\prime}$ at energy density $e=\epsilon\left(x^{*}\right)$. This follows from $\rho(e)=\frac{d}{d e} \epsilon^{-1}(e)\left(\epsilon^{-1}\right.$ denotes the inverse function of $\epsilon$ ) and differentiating the identity $\epsilon \circ \epsilon^{-1}(e)=e$ once and twice w.r.t. e. Applying Poisson's summation to the series in 4.20 leads to

$$
P(W=0, t) \asymp\left|\sum_{\xi \in 2 \pi N \mathbb{Z}}(\widehat{\chi} * \widehat{\alpha})(N \omega t+\xi)\right|^{2}
$$



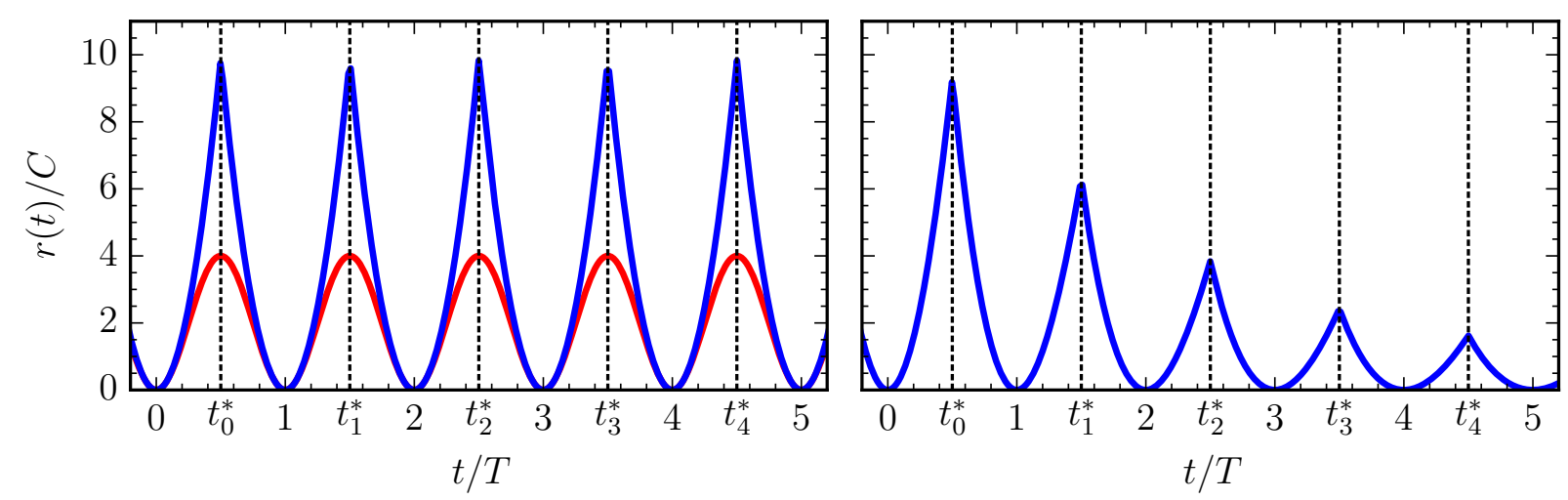

Figure 4.4: Zero work probability rate function $r(t)$ (blue and red lines) in a double quench setup when the post-quench Hamiltonian has constant density of states $\rho=1 / \omega$ (left), and density of states with constant slope $\Omega=-\rho^{\prime} / \rho^{3} \neq 0$ (right). Pre-quench ground state has Gaussian (variance $C$ ) distributed overlaps with the post-quench energy eigenstates (solid blue line). Non-analytic behavior of $r(t)$ occurs at odd-integer multiples $t_{n}^{*}$ of $T=$ $2 \pi / \omega$ (dotted black line) according to Eqs. (4.15) (left), and (4.23) (right), respectively. Non-vanishing $\Omega$ yields quadratic decay (right) of the rate function envelope caused by the dephasing among the post-quench energy modes. (The numerical value of $\Omega C T$ is $1 / 2$ in the right plot.) In comparison, the zero work probability rate function after a sudden shift of the center of an harmonic oscillator by $\Delta$ (solid red line) is analytic for all times, cf. (4.18). For this quench the overlaps are Poisson distributed with variance $\Delta^{2} / 2=C$. The difference between red and blue come from details in the tails of the overlap function. Hence, mean and variance of the overlap distribution is not sufficient to infer DPT-II, the existence and absence of DPT-II is sensitive to the full overlap rate function. 
where the hat denotes Fourier transformation $\widehat{\alpha}(p)=\int_{\mathbb{R}} d x \alpha(x) e^{-i p x} \propto \exp \left[\frac{i p^{2}}{2 N \Omega t}\right]$, and the star denotes convolution. Equations (4.20) and 4.21) are the analogs of Eqs. (4.9) and 4.10), respectively, and the latter are obtained from the former by setting $\Omega=0$. The crucial difference between (4.10) and (4.21) comes from the time dependence of $\widehat{\chi} * \widehat{\alpha}$. Denoting the time-dependent rate function of $(\widehat{\chi} * \widehat{\alpha})(N p) \asymp e^{-N g_{t}(p)}$ by $g_{t}(p)$, one infers the rate function

$$
r(t)=2 \min _{\xi \in 2 \pi \mathbb{Z}} \Re g_{t}(\omega t+\xi)=2 \min _{n \in \mathbb{Z}} \Re g_{t}(\omega(t+n T))
$$

of $P(W=0, t)$.

As in Eq. (4.13), the switching of the branch in 4.22 where the minimum is attained, occurs at the intersection of two terms of the form $\Re g_{t}(\omega t+\xi)$ with consecutive values of $\xi \in 2 \pi \mathbb{Z}$. These critical times are candidates for DPT-II.

The fact that the post-quench spectrum is no longer equidistant for $\Omega \neq 0$ entails that $r(t)$ is no longer exactly periodic. Roughly speaking, the convolution of $\widehat{\chi}$ with $\widehat{\alpha}$ can be viewed as blurring the distribution $\widehat{\chi}$ with 'Gaussian noise' (ignoring the imaginary unit in the exponent of $\widehat{\alpha}$ ). As the variance of this noise increases linear in time, the rate function $\Re g_{t}$ of the blurred distribution $|\widehat{\chi} * \widehat{\alpha}|$ decays inversely quadratic for late times. Physically, this decay can be viewed as the result of a dephasing effect between the post-quench eigenmodes. The following concrete examples serve as an illustration.

Example (Gaussian overlaps, revisited, cf. Fig. 4.4). As in the above example, we consider Gaussian overlaps $\chi$ with rate function $f(x)=\frac{1}{2} x^{2} / C$ such that $\widehat{\chi}(N p)=\exp (-N g(p))$ with $g(p)=\frac{1}{2} C p^{2}$. This time we allow for non-zero $\Omega$ and $\widehat{\alpha}(N p) \asymp \exp \left(-N \frac{1}{2 i \Omega t} p^{2}\right)$. The convolution $(\widehat{\chi} * \widehat{\alpha})(N p) \asymp \exp \left[-N g_{t}(p)\right]$ has a quadratic rate function $g_{t}(p)=\frac{1}{2} C_{t} p^{2}$, where $C_{t}=\left(\frac{1}{C}+i \Omega t\right)^{-1}$. According to 4.22 $r(t)=\min _{\xi \in 2 \pi \mathbb{Z}} \Re\left(C_{t}\right)(\omega t+\xi)^{2}$. Two consecutive branches, associated to $\xi_{n}=2 \pi n$ and $\xi_{n+1}=2 \pi(n+1)$, say, intersect uniquely at $t_{n}^{*}=(n+1 / 2) T$, and the minimization can be performed explicitly,

$$
r(t)=\frac{C}{1+(C \Omega t)^{2}} \omega^{2}[t]_{[-T / 2, T / 2]}^{2},
$$

where $T=2 \pi / \omega$ (recall, $[t]_{[-a, a]}=t-2 a\left\lfloor\frac{t}{2 a}+\frac{1}{2}\right\rfloor$ denotes the projection onto the interval $[-a, a]$, cf. Eq. 4.14). This shows explicitly how non-zero $\Omega$ entails quadratic decay of the late time envelope of $r(t)$, cf. Fig. 4.4. In the limiting case $\Omega=0$, Eq. 4.15) is recovered.

Example (FC-TFIM, cf. Fig. 4.5). Now, we return to the FC-TFIM with Hamiltonian (4.3) in the large $N$ limit. Essentially, we argue that the Loschmidt rate function after a quench in the external field $\Gamma_{i} \mapsto \Gamma_{f}$ has the form of Eq. 4.23 of the previous example. To this end, we show that the two assumptions 4.7a and 4.7b are fulfilled. We use the semiclassical techniques in the effective description in terms of $H_{\text {eff }}$, cf. Eq. (4.4), to give explicit expressions for (i) the variance $C$ of the post-quench spectral overlaps with the pre-quench ground state (cf. Eq. (4.27)), and (ii) the parameters $\omega=1 / \rho$ and $\Omega-\rho^{\prime} / \rho^{3}$ (cf. Eq. 4.28). Phase space coordinates $z=\left(n_{+}, p\right)$ take values in $[0,1] \times[-\pi, \pi]$.

First, in the large $N=\hbar_{\text {eff }}^{-1}$ limit the Wigner function $W_{\mathrm{GS}}$ of the pre-quench ground state is localized at

$$
z_{0}=\left(n^{*}, 0\right), \quad \text { where } \quad n^{*}= \begin{cases}\frac{1}{2} \sqrt{1-4 \Gamma_{i}^{2}}, & \Gamma_{i}<1 / 2 \\ 0, & \Gamma_{i}>1 / 2\end{cases}
$$




\section{Dynamical Phase Transitions}

with covariance matrix

$$
\frac{C_{\mathrm{GS}}}{N}=\frac{1}{2 N}\left(\begin{array}{cc}
g_{2}^{-1} & 0 \\
0 & g_{2}
\end{array}\right), \quad \text { where } \quad g_{2}= \begin{cases}\frac{1}{2} \sqrt{1-4 \Gamma_{i}^{2}} / \Gamma_{i}^{2}, & \Gamma_{i}<1 / 2 \\
\sqrt{4-2 / \Gamma_{i}}, & \Gamma_{i}>1 / 2\end{cases}
$$

cf. Eq. (11) of [1], and Eq. 3.42). The overlap of the pre-quench ground state $\left|\psi_{\mathrm{GS}}\right\rangle$ with the post-quench eigenstate $|E\rangle$ is

$$
\left|\left\langle E \mid \psi_{\mathrm{GS}}\right\rangle\right|^{2}=\int d z W_{\mathrm{GS}}(z) W_{|E\rangle\langle E|}(z) \approx \int d z W_{\mathrm{GS}}(z) \delta\left[H_{\mathrm{eff}}(z)-E\right]
$$

where $W_{|E\rangle\langle E|}$ is the Wigner function of the eigenstate of the post quench Hamiltonian $\left.H_{\mathrm{eff}}\right|_{\Gamma=\Gamma_{f}}$. The first equality is an exact identity. The second approximate equality is the semiclassical result (2.8) in the limit $N \rightarrow \infty$. In view of (4.24), $W_{\mathrm{GS}}$ is approximated by the Gaussian function $\mathcal{N}_{z_{0}}^{C_{\mathrm{GS}}} / N(z) \propto \exp \left[-N\left(z-z_{0}\right) C_{\mathrm{GS}}^{-1}\left(z-z_{0}\right) / 2\right]$ with mean $z_{0}$ and variance $C_{\mathrm{GS}} / N$. By straightforward computation,

$$
\begin{aligned}
\left|\left\langle E \mid \psi_{\mathrm{GS}}\right\rangle\right|^{2} & \approx \int \mathcal{N}_{z_{0}}^{C_{\mathrm{GS}} / N}(z) \delta\left[H_{\mathrm{eff}}(z)-E\right] \\
& \approx \int \mathcal{N}_{z_{0}}^{C_{\mathrm{GS}} / N}(z) \delta\left[H_{\mathrm{eff}}\left(z_{0}\right)+\nabla H_{\mathrm{eff}}\left(z_{0}\right) \cdot\left(z-z_{0}\right)-E\right] \\
& =\mathcal{N}_{H_{\mathrm{eff}} H_{\mathrm{eff}}\left(z_{0}\right) C_{\mathrm{GS}} \nabla H_{\mathrm{eff}}\left(z_{0}\right) / N}(E) .
\end{aligned}
$$

It follows that the overlap is approximately Gaussian with covariance of order $1 / N$. Note that the overlap is maximal at the post-quench energy $E^{*}:=\left.H_{\mathrm{eff}}\left(z_{0}\right)\right|_{\Gamma=\Gamma_{f}}$. We identify the index of the eigenenergy $E^{*}$ with $x^{*}=\int_{-\infty}^{E^{*}} \rho(E) d E$, where $\rho$ is the density of states of the post-quench Hamiltonian $H_{\text {eff. }}$. The density of states $\rho$ of $H_{\text {eff }}$ can be approximated in the large $N$ limit as 8

$$
\rho(E)=\frac{1}{2 \pi} \int d^{2} z \delta\left[H_{\mathrm{eff}}(z)-E\right]
$$

cf. Eq. 2.4a and Fig. 4.5. Finally, we change the energy variable $E$ to the index variable $x=n / N$ via $E(x)=E^{*}+\epsilon^{\prime}\left(x^{*}\right)\left(x-x^{*}\right)$. Recall, that $\epsilon^{\prime}\left(x^{*}\right)=1 / \rho\left(E^{*}\right)$, and denote $\rho^{*}=\rho\left(E^{*}\right)$, then

$$
|\chi(x)|^{2}=\mathcal{N}_{x^{*}}^{C / N}(x), \quad \text { where } \quad C=\left(\rho^{*}\right)^{2}\left[\nabla H_{\mathrm{eff}}\left(z_{0}\right)\right] \cdot C_{\mathrm{GS}} \cdot\left[\nabla H_{\mathrm{eff}}\left(z_{0}\right)\right],
$$

in accordance to Eq. 4.7b, cf. Fig. 4.5 .

Second,

$$
\omega=1 / \rho\left(E^{*}\right) \quad \text { and } \quad \Omega=-\rho^{\prime}\left(E^{*}\right) / \rho\left(E^{*}\right)^{3}
$$

follow directly from (4.26) and $E^{*}=\left.H_{\mathrm{eff}}\left(z_{0}\right)\right|_{\Gamma=\Gamma_{f}}$. The discussion of Eq. 2.5 shows that $T=$ $2 \pi / \omega=2 \pi \rho^{*}$ is indeed the period of the classical trajectory of the post-quench Hamiltonian $H_{\text {eff }}$ starting in $z_{0}$. Hence, the time between two consecutive dynamical phase transitions at $t_{n}^{*}=(n+1 / 2) T$ agrees with the period of the order parameter oscillation after the quench, cf. [2].

The semiclassical analytic prediction Eqs. (4.23), (4.27) and (4.28) agrees well with ED numerical data for $N=10^{3}$. Two different quenches, one within the ferromagnetic phase, and one from the

${ }^{8}$ An equivalent expression,

$$
\rho(E)=\Re \frac{1}{\pi} \int_{-1 / 2}^{1 / 2} d n{\sqrt{\left[E-H_{\text {eff }}(n, 0)\right]\left[H_{\text {eff }}(n, \pi)-E\right]}}^{-1}
$$

for $E \in\left[\min _{n} H_{e} f f(n, 0), \max _{n} H_{\text {eff }}(n, \pi)\right]$, has been derived with a Moyal-star approach in 176 . 
ferromagnetic phase to the paramagnetic phase, are shown in Fig. 4.5. Irrespective of whether the quench crosses the equilibrium critical point, DPT-II occur in agreement with the analytic prediction.
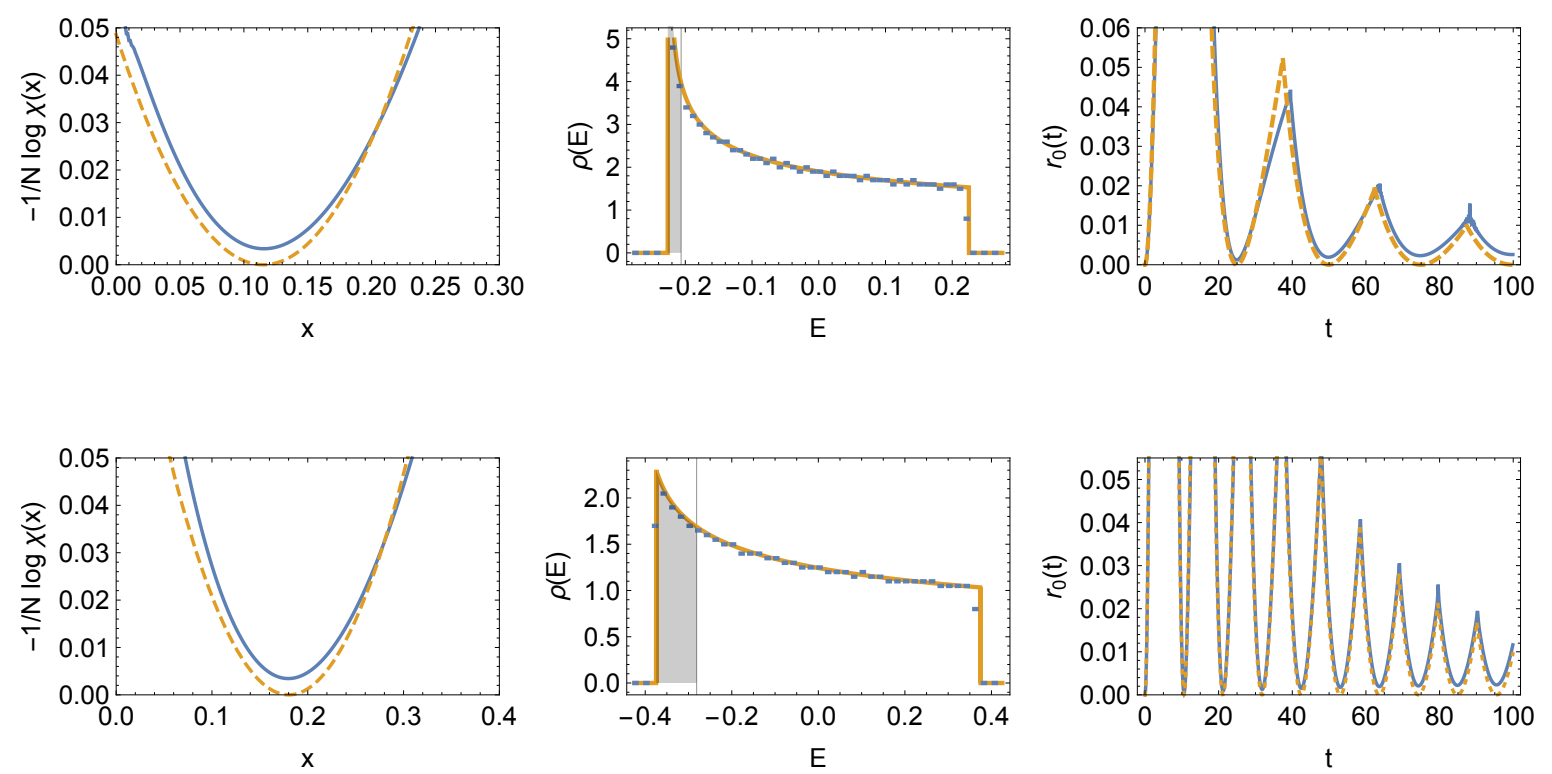

Figure 4.5: Quench $\Gamma_{i}=0.25 \mapsto \Gamma_{f}=0.45$ (top), and $\Gamma_{i}=0.25 \mapsto \Gamma_{f}=0.75$ (bottom), cf. Fig. 4.2. Rate function $-\frac{1}{N} \log \chi(x)$ of the overlap $\chi(n / N)=\left|\left\langle\psi_{0} \mid E_{n}\right\rangle\right|^{2}$ between the pre-quench ground state $\left|\psi_{0}\right\rangle$ and the post-quench eigenfunctions $\left|E_{n}\right\rangle$ as a function of the normalized index $x=n / N$ (left). Exact diagonalization results (blue line) are obtained for $N=10^{3}$ spins, cf. (4.3); analytic prediction (orange dashed line) according to (4.27). Normalized density of states of the post-quench Hamiltonian for $\Gamma_{f}=0.75$ (middle) obtained by ED (blue line), and according to Eq. 4.26) (orange line). The vertical line marks the position of $E^{*}=H_{\text {eff }}\left(z_{0}\right)$. The size of the shaded area is $x^{*}$, the index where $\chi(x)$ attains its maximum. Loschmidt rate function $-\frac{1}{N} \log \left|\left\langle\psi_{0} \mid \psi_{t}\right\rangle\right|^{2}$ (right) obtained with ED (solid blue line), and according to the analytic prediction Eqs. 4.23, 4.27) and 4.28) (dashed orange line).

\subsection{Discussion}

DPT-II, i.e. the occurrence of non-analytic behavior in the Loschmidt return rate function, can strictly speaking only occur in infinite systems. Because for finite system sizes the expansion of the Loschmidt echo in the energy eigenbasis yields a finite, and thus, analytic sum. Numerical data for large systems can indicate the occurrence of non-analytic cusps in the Loschmidt return rate function. But numerics alone cannot prove non-analyticities. In this chapter, we have studied the fully connected transverse field Ising model (FC-TFIM) of $N$ spins to address the question of DPT-II by analytic means in the thermodynamic limit $N \rightarrow \infty$. Analytic considerations are facilitated by the effective semiclassical description of the spin model.

We have presented two different approaches to approximate the Loschmidt rate function in terms of semiclassical quantities in the large $N$ limit. First, in terms of the Wigner quasi-probability 


\section{Dynamical Phase Transitions}

distribution of the pre-quench ground state. And second, in terms of the overlap probability of the ground state with the post-quench eigenstates. Both arguments show that the precise form of the Loschmidt rate function, and hence, the existence of DPT-II, depends on the details of the exponentially suppressed tails of the respective semiclassical distribution. Furthermore, in both arguments the mathematical mechanism leading to non-analyticities is the non-analytic nature of a minimization problem.

Expanding the overlap rate function to second order around its minimum amounts to approximating the tail of the overlap probability by a Gaussian function. This approximation is insignificant for, e.g., expectation values of typical observables, which are insensitive to exponentially suppressed large deviations from the mean. However, the presence and absence of non-analytic behavior in the Loschmidt return function is sensitive to those large deviations. We stress once more that the existence of DPT-II depends on the full overlap rate function, and not just on the local properties of the rate function around its minimum. In particular, approximating the overlap tail by a Gaussian function can erroneously predict a DPT-II, while the correct Loschmidt rate function is perfectly analytic. A quench in the exactly solvable harmonic oscillator is an explicit exemplification of this fact. Hence, the Gaussian approximation can only yield candidates for critical times of DPT-II.

In the FC-TFIM, the Gaussian approximation of the overlap probability and the semiclassical approximation of the density of states predicts candidates of DPT-II at equally spaced times with the period of the order parameter oscillation. Good agreement of the analytic result with ED data, cf. Fig. 4.5, justifies the semiclassical approximations. Notwithstanding, the derivation has to be considered cautiously, due to the aforementioned approximation of the exponential tails.

Publication $[2$ is a numerical study of DPT in the FC-TFIM. Even though the permutation invariance of the FC-TFIM allows to study the system numerically by ED for a considerably large number of spins (up to $N=1200$ in Ref. [2]), the evidence of a DPT-II is mathematically non-rigorous. Based on the numerical observations in Ref. 2] two types of DPT-II scenarios are discriminated, and are coined regular and anomalous DPT-II, respectively. First, for large quenches, cusps in the Loschmidt rate function $r(t)$ are regularly space at critical times, and the first cusp occurs before the first minimum of $r(t)$. Schematically, this is the situation of Fig. 4.3 . and constitutes the phase of regular cusps. Second, for small quenches the Loschmidt rate function still shows non-analytic cusps, but the first cusp occurs after the first minimum of $r(t)$. The first cusp is preceded by at least one analytic maximum, cf. Figs. 3 to 5 in [2]. The smaller the quench is, the more analytic maxima precede the first non-analytic cusp. After the first cusp further cusps occur regularly spaced similar to the regular case. This constitutes the phase of anomalous cusps.

Ref. [2] observes that quenches with regular and anomalous cusps are separated by the critical line of a DPT-I in the dynamical phase diagram. More precisely, the infinite time average of the order parameter is positive for quenches that show anomalous cusps, while it vanishes for quenches that yield regular cusps.

The behavior of the anomalous cusps being preceded by analytic maxima, goes beyond the analytic prediction discussed in this chapter. In particular, such a behavior is not explained by (4.22). Similar to the example of the harmonic oscillator, this is another instance of the fact that the analysis presented in this chapter can only yield candidates for critical times at which DPT-II can occur. A more careful analysis of the exponential tails in the overlap function and the density of states beyond the presentation of this chapter is required in this case. 


\subsection{Publication [2]}

The author's contribution. The idea to study the relation between the DPT-I and DPT-II in the FC-TFIM is due to Jad Halimeh and Valentin Zauner-Stauber, and is motivated by the results of their previous paper Ref. [148]. The author of this thesis has produced all figures in Ref. [2]. Numerical exact diagonalization data was produced with two independent implementations. One implementation is due to the author of this thesis, the other implementation is by Nils Abeling. The author of this thesis has participated in scientific discussions, and contributed to the writing and proofreading of the manuscript. Sec. II B. was written by the author of this thesis.

Copyright. (C)2017 American Physical Society 


\title{
Anomalous dynamical phase in quantum spin chains with long-range interactions
}

\author{
Ingo Homrighausen, ${ }^{1}$ Nils O. Abeling, ${ }^{1}$ Valentin Zauner-Stauber, ${ }^{2}$ and Jad C. Halimeh ${ }^{3,4,5}$ \\ ${ }^{1}$ Universität Göttingen, Institut für Theoretische Physik, Friedrich-Hund-Platz 1, 37077 Göttingen, Germany \\ ${ }^{2}$ Vienna Center for Quantum Technology, University of Vienna, Boltzmanngasse 5, 1090 Wien, Austria \\ ${ }^{3}$ Physics Department and Arnold Sommerfeld Center for Theoretical Physics, \\ Ludwig-Maximilians-Universität München, D-80333 München, Germany \\ ${ }^{4}$ National Institute for Theoretical Physics (NITheP), Stellenbosch 7600, South Africa \\ ${ }^{5}$ Institute of Theoretical Physics, Department of Physics, University of Stellenbosch, Stellenbosch 7600, South Africa
}

(Received 5 April 2017; published 26 September 2017)

\begin{abstract}
The existence or absence of nonanalytic cusps in the Loschmidt-echo return rate is traditionally employed to distinguish between a regular dynamical phase (regular cusps) and a trivial phase (no cusps) in quantum spin chains after a global quench. However, numerical evidence in a recent study (J. C. Halimeh and V. Zauner-Stauber, arXiv:1610.02019) suggests that instead of the trivial phase, a distinct anomalous dynamical phase characterized by a novel type of nonanalytic cusps occurs in the one-dimensional transverse-field Ising model when interactions are sufficiently long range. Using an analytic semiclassical approach and exact diagonalization, we show that this anomalous phase also arises in the fully connected case of infinite-range interactions, and we discuss its defining signature. Our results show that the transition from the regular to the anomalous dynamical phase coincides with $\mathbb{Z}_{2}$-symmetry breaking in the infinite-time limit, thereby showing a connection between two different concepts of dynamical criticality. Our work further expands the dynamical phase diagram of long-range interacting quantum spin chains, and can be tested experimentally in ion-trap setups and ultracold atoms in optical cavities, where interactions are inherently long range.
\end{abstract}

DOI: 10.1103/PhysRevB.96.104436

\section{INTRODUCTION}

Dynamical phase transitions have recently been the subject of intense theoretical and experimental investigation. Most commonly, they fall into two main types, both of which involve a quench where a control parameter in the system Hamiltonian is abruptly switched from some initial value to a final one, subsequently throwing the system out of equilibrium. The first kind of dynamical phase transition (DPT-I) [1-5] is of the Landau type: one waits for the system to relax into a (quasi)steady state and extracts a suitable order parameter, usually that associated with spontaneous symmetry breaking in the system at equilibrium. This is done as a function of the final value of the quench-control parameter, and if a nonanalyticity arises in this function, then a DPT-I has occurred in the system.

A second type of dynamical phase transition is the DPT-II $[6,7]$ in which nonanalyticities in time, or lack thereof, in the Loschmidt-echo return rate

$$
r(t)=-\lim _{N \rightarrow \infty} \frac{1}{N} \ln \left|\left\langle\psi_{0}\left|e^{-i \hat{\mathcal{H}} t}\right| \psi_{0}\right\rangle\right|^{2}
$$

characterize different phases, with prequench ground state $\left|\psi_{0}\right\rangle$, system size $N$, and post-quench Hamiltonian $\hat{\mathcal{H}}$. In the context of the DPT-II, an analogy [6] is made between the thermal partition function and the Loschmidt echo $\left\langle\psi_{0}\left|e^{-i \hat{\mathcal{H}} t}\right| \psi_{0}\right\rangle$ or, equivalently, between the thermal free energy and the Loschmidt-echo return rate $r(t)$, where evolution time is now interpreted as a complex inverse temperature. Consequently, if the Loschmidt-echo return rate exhibits nonanalyticities in evolution time after a quench, this is analogous to nonanalyticities in the free energy of a system in equilibrium, which is the hallmark of an equilibrium phase transition [8]. This DPT-II, first classified in the seminal work of Ref. [6] for the one-dimensional nearest-neighbor transverse-field Ising model (NN-TFIM), has been studied both analytically [3,7,917] and numerically [4,18-28] in various models, and has also been experimentally observed [29-31]. Even though for certain quenches [6] the critical final value of the quenching parameter that separates the phase with cusps from that with no cusps coincides with the equilibrium critical point of the model, this is not always the case [12,23], and in general the dynamical critical point separating such dynamical phases is different from its equilibrium counterpart.

In Fig. 1 we show, in the context of the DPT-II for quenches from zero field strength, the dynamical phase diagram of the one-dimensional long-range transverse-field Ising model (LRTFIM) given by the Hamiltonian

$$
\hat{\mathcal{H}}(\Gamma)=-\frac{J}{2 \mathcal{N}} \sum_{i \neq j}^{N} \frac{1}{|i-j|^{\alpha}} \hat{S}_{i}^{z} \hat{S}_{j}^{z}-\Gamma \sum_{i} \hat{S}_{i}^{x},
$$

where $\hat{S}_{i}^{a}, a=x, y, z$, are the spin- $\frac{1}{2}$ operators on site $i$, $J>0$ is the spin-spin coupling constant, $\Gamma$ is the strength of the transverse magnetic field, $\alpha \geqslant 0$, and $\mathcal{N}$ is the Kac normalization [32] given by

$$
\mathcal{N}=\frac{1}{N-1} \sum_{i \neq j}^{N} \frac{1}{|i-j|^{\alpha}}=\frac{2}{N-1} \sum_{n=1}^{N} \frac{N-n}{n^{\alpha}},
$$

which guarantees energy-density intensivity for $\alpha \leqslant 1$. The part of this diagram at $\alpha=0$ is the main result of this work. The part of this phase diagram for $\alpha>1$ has been constructed using matrix product state (MPS) techniques for infinite systems, a method known as iMPS [18,33-37]. In the limit $\alpha \rightarrow \infty$, the nearest-neighbor result [6] is obtained. As can be seen in Fig. 1, quenching from zero field strength to above a certain dynamical critical value sets the system in a regular dynamical phase characterized by the appearance of an infinite 


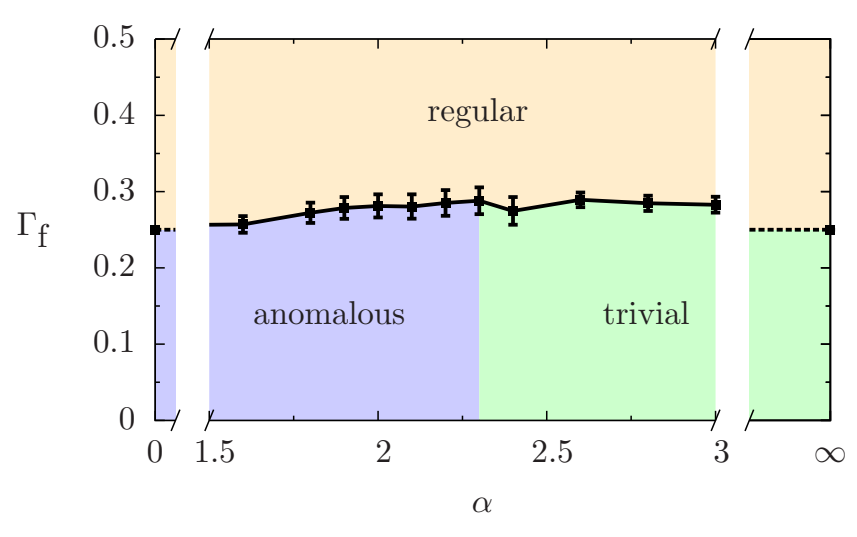

FIG. 1. The dynamical phase diagram of the one-dimensional LR-TFIM (2) after a global quench with initial field strength $\Gamma_{\mathrm{i}}=0$, showing three distinct dynamical phases: regular, anomalous, and trivial (see main text). The dynamical critical line is marked in solid black. The results for the nonintegrable model are obtained using iMPS [18], while the dynamical critical point for the NN-TFIM $(\alpha \rightarrow$ $\infty)$ is known analytically [6]. The phase diagram for the FC-TFIM $(\alpha=0)$ is the main result of this work.

sequence of cusps with the first cusp appearing before the first minimum in the Loschmidt-echo return rate. These cusps become sharper and temporally less separated with increasing quench strength $[6,18]$. However, for sufficiently long-range interactions ( $\alpha \lesssim 2.3$ ), a new anomalous dynamical phase [18] appears whose defining signature is that cusps appear only after the first minimum in the return rate. In contrast to their regular counterparts, the anomalous cusps separate less in time from each other with decreasing quench strength, with more smooth maxima emerging in the return rate before their onset. In fact, numerical results [18] suggest that these cusps arise for arbitrarily small quenches, even though in the framework of iMPS and time-dependent density matrix renormalization group [38-45] ( $t$-DMRG) techniques entanglement buildup prevents access to long-enough evolution times that would be necessary to see the onset of these anomalous cusps for extremely weak quenches.

In this paper, we turn our attention to the analytically tractable fully connected transverse-field Ising model (FCTFIM), and investigate the nature of the anomalous phase in a semiclassical approach [1]. The advantage of this is twofold: (i) In iMPS, it is intrinsically difficult to include the Kac normalization to ensure intensivity of the energy density for $\alpha \leqslant 1$, whereas the FC-TFIM allows for investigating the anomalous phase with exact diagonalization (ED) and semiclassical techniques. ED is a technique which is fundamentally different from iMPS methods, therefore, it additionally provides an alternate venue to study the anomalous phase. (ii) Moreover, from an intuitive point of view, it is logical to consider the limit of infinite-range interactions since it appears that the anomalous phase occurs only for interactions that are sufficiently long range.

The remainder of the paper is organized as follows: In Sec. II we review the FC-TFIM and use a semiclassical treatment to derive the infinite-time average of the $\mathbb{Z}_{2}$ order parameter and its oscillation period. In Sec. III we present and discuss our results obtained from ED, characterize the anomalous phase, and discuss the connection between the cusps in the return rate and the $\mathbb{Z}_{2}$ order parameter. We conclude in Sec. IV.

\section{FULLY CONNECTED TRANSVERSE-FIELD ISING MODEL}

\section{A. Model and quench}

The one-dimensional FC-TFIM is described by taking the $\alpha=0$ limit of (2),

$$
\hat{\mathcal{H}}(\Gamma)=-\frac{J}{2 N} \sum_{i \neq j}^{N} \hat{S}_{i}^{z} \hat{S}_{j}^{z}-\Gamma \sum_{i} \hat{S}_{i}^{x}-\epsilon \sum_{i} \hat{S}_{i}^{z},
$$

where we have additionally introduced a $\mathbb{Z}_{2}$-symmetrybreaking term with $\epsilon$ a small positive longitudinal field of $\mathscr{O}(1 / N)$ because we treat finite-size systems only and spontaneous symmetry breaking is a feature of the thermodynamic limit. The FC-TFIM has an equilibrium quantum critical point [46] at $\Gamma_{\mathrm{c}}^{\mathrm{e}}=J / 2$. Hence, in the ground state of (4) the longitudinal magnetization is positive for $\Gamma<\Gamma_{\mathrm{c}}^{\mathrm{e}}$ and vanishes for $\Gamma>\Gamma_{\mathrm{c}}^{\mathrm{e}}$.

We are interested in the DPT-II and its corresponding dynamical phases in the FC-TFIM while using $\Gamma$ as the quench-control parameter. In the following, we shall prepare our system in the ground state $\left|\psi_{0}\right\rangle$ of $\hat{\mathcal{H}}\left(\Gamma_{\mathrm{i}}\right)$, and then at time $t=0$, the field strength is suddenly switched from $\Gamma_{\mathrm{i}}$ to $\Gamma_{\mathrm{f}} \neq \Gamma_{\mathrm{i}}$, leading to time evolving the system under $\hat{\mathcal{H}}\left(\Gamma_{\mathrm{f}}\right)$ and subsequently discerning from the return rate what dynamical phase our system is in from the perspective of the DPT-II. The DPT-I in this model was first studied in Ref. [1]. Moreover, it was argued that there is an equivalence $[4,18]$ between the DPT-I and DPT-II in the LR-TFIM, and also in the FC-TFIM [4]. Nevertheless, to the best of our knowledge, the anomalous phase has not been previously investigated outside of Ref. [18], which does so numerically in the context of the LR-TFIM for $\alpha>1$.

\section{B. Semiclassical equations of motion}

The period of the $\mathbb{Z}_{2}$ order parameter $\hat{s}^{z}(t)=\sum_{i} \hat{S}^{z}(t) / N$ can be computed in an effective semiclassical picture [1]. To leading order in the mean-field limit $N \rightarrow \infty$, the post-quench magnetization expectation value $\left\langle\hat{s}^{z}\right\rangle=s(t)+\mathscr{O}(1 / N)$ evolves according to Hamilton's equations of motion $\dot{s}(t)=$ $\partial_{p} H_{\text {eff }}$ and $\dot{p}(t)=-\partial_{s} H_{\text {eff }}$, with the effective Hamiltonian

$$
H_{\mathrm{eff}}(s, p)=-\frac{J}{2} s^{2}-\frac{\Gamma_{\mathrm{f}}}{2} \sqrt{1-4 s^{2}} \cos p,
$$

and initial condition

$$
\begin{gathered}
s(0)= \begin{cases}0, & \text { if } \Gamma_{\mathrm{i}}>\Gamma_{\mathrm{c}}^{\mathrm{e}} \\
\sqrt{\frac{1}{4}-\Gamma_{\mathrm{i}}^{2},} & \text { if } \Gamma_{\mathrm{i}}<\Gamma_{\mathrm{c}}^{\mathrm{e}}\end{cases} \\
p(0)= \begin{cases}0, & \text { if } \Gamma_{\mathrm{i}} \neq 0 \\
-\pi / 2, & \text { if } \Gamma_{\mathrm{i}}=0 .\end{cases}
\end{gathered}
$$




$$
\Gamma_{\mathrm{i}}=0
$$

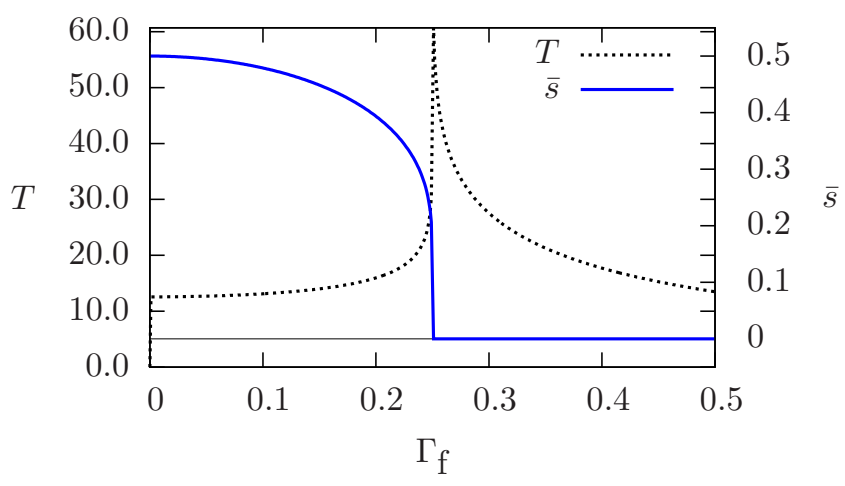

FIG. 2. The periodicity of the order parameter (dotted black line) in the FC-TFIM for a quench from $\Gamma_{\mathrm{i}}=0$ to $\Gamma_{\mathrm{f}}$, derived in a semiclassical approach. This periodicity is also that of the nonanalytic cusps arising in the Loschmidt-echo return rate (1). The period diverges at the dynamical critical point $\Gamma_{c}^{d}=0.25$, i.e., at the point where the infinite-time average of the longitudinal magnetization (solid blue line) is nonanalytic as a function of $\Gamma_{\mathrm{f}}$. The critical point $\Gamma_{\mathrm{c}}^{\mathrm{d}}$ also separates the anomalous and regular phases.

Henceforth, we choose units of time in which $J=1$. The period of the classical orbit is

$$
\begin{aligned}
T & =2 \int_{s_{-}}^{s_{+}} \frac{d s}{\partial_{p} H_{\mathrm{eff}}} \\
& =2 \int_{s_{-}}^{s_{+}} \frac{d s}{\sqrt{\left(\frac{1}{4}-s^{2}\right) \Gamma_{\mathrm{f}}^{2}-\left(E+\frac{1}{2} s^{2}\right)}}
\end{aligned}
$$

and the average magnetization along this orbit is

$$
\begin{aligned}
\bar{s} & =\frac{1}{T} \int_{0}^{T} s(t) d t \\
& =\frac{2}{T} \int_{s_{-}}^{s_{+}} \frac{s d s}{\sqrt{\left(\frac{1}{4}-s^{2}\right) \Gamma_{\mathrm{f}}^{2}-\left(E+\frac{1}{2} s^{2}\right)}}
\end{aligned}
$$

where the integration bounds $s_{-}<s_{+}$are the turning points of the trajectory $s(t)$, and the energy

$$
E=H_{\mathrm{eff}}(s(0), p(0))
$$

is conserved. For $\Gamma<\Gamma_{\mathrm{c}}^{\mathrm{e}}$, the Hamiltonian (5) has a hyperbolic fixed point at $(s, p)=(0,0)$, whose stable directions are connected to the unstable directions by two homoclinic orbits. The homoclinic orbits separate closed $\mathbb{Z}_{2}$-invariant orbits (i.e., orbits that are invariant under $s \mapsto-s$ ) from closed orbits that are not $\mathbb{Z}_{2}$ invariant. As pointed out in Ref. [1], this leads to a DPT-I at

$$
\Gamma_{\mathrm{c}}^{\mathrm{d}}\left(\Gamma_{\mathrm{i}}\right)=\left(\Gamma_{\mathrm{c}}^{\mathrm{e}}+\Gamma_{\mathrm{i}}\right) / 2
$$

For quenches to $\Gamma_{\mathrm{f}}=\Gamma_{\mathrm{c}}^{\mathrm{d}}$ the initial condition (6) lies on a homoclinic orbit and $s(t)$ approaches $s=0$ exponentially in time, i.e., the period (8) of $s(t)$ diverges at $\Gamma_{\mathrm{c}}^{\mathrm{d}}$ as shown in Fig. 2. For quenches to $\Gamma_{\mathrm{f}}>\Gamma_{\mathrm{c}}^{\mathrm{d}}$, the orbit is $\mathbb{Z}_{2}$ symmetric and $s(t)$ oscillates around zero such that the infinite-time average

$$
\bar{s}=\lim _{t \rightarrow \infty} \lim _{N \rightarrow \infty} \frac{1}{N t} \int_{0}^{t} d t^{\prime} \sum_{i}\left\langle\hat{S}_{i}^{z}\left(t^{\prime}\right)\right\rangle
$$

vanishes. Note that the limit $N \rightarrow \infty$ has to be taken before the limit $t \rightarrow \infty$ in order to obtain the semiclassical result (9). In contrast, for $\Gamma_{\mathrm{f}}<\Gamma_{\mathrm{c}}^{\mathrm{d}}$, the orbit is not $\mathbb{Z}_{2}$ symmetric and the infinite-time average takes a nonzero value (cf. Fig. 2).

\section{RESULTS AND DISCUSSION}

We shall now present our results on the two distinct phases (regular and anomalous) of the DPT-II in the FC-TFIM, and argue that they are intimately related to the phases of the DPT-I in this model through sharing the same critical point $\Gamma_{\mathrm{c}}^{\mathrm{d}}$. Traditionally, the DPT-II is known to give rise to two phases: one with (regular) cusps for quenches across the DPTII critical point, and a second with no cusps in the return rate for quenches not crossing it. In Ref. [6], this was demonstrated in the case of the NN-TFIM, where it can be analytically shown that the DPT-II critical point is $\Gamma_{\mathrm{c}}^{\mathrm{e}}$. Much like the case of the NN-TFIM, the return rate in the FC-TFIM also shows regular cusps for quenches across $\Gamma_{c}^{d}$, as shown in Fig. 3 for $\Gamma_{i}=0$. In agreement with previous results $[4,6,18]$, these cusps occur before the first minimum of the return rate and beyond. Also, the period of these cusps matches that of the order parameter at longer times and decreases with quench strength while the cusps themselves get sharper.

In the case of the NN-TFIM, cusps in the return rate are absent [6] for quenches below $\Gamma_{\mathrm{c}}^{\mathrm{e}}$, and the return rate is fully analytic. This has also been observed in Ref. [18] to be the case for the LR-TFIM with sufficiently short-range interactions $\alpha \gtrsim$ 2.3. However, for longer-range interactions, the return rate does exhibit new kinds of cusps that are qualitatively different in their behavior from their regular counterparts. These cusps characterize the anomalous dynamical phase, defined by a Loschmidt-echo return rate that displays nonanalyticities only after its first minimum. In fact, it can be shown in iMPS that these anomalous cusps are caused by level crossings within the set of dominant eigenvalues of the MPS transfer matrix, which is qualitatively different from the set responsible for the manifestation of the regular cusps and which is dominant for quenches above the DPT-II critical point. In good agreement with iMPS data for the LR-TFIM, our ED results in Fig. 4 for the FC-TFIM show such anomalous cusps in the return rate for quenches below $\Gamma_{\mathrm{c}}^{\mathrm{d}}$, which, unlike the case of the NN-TFIM, is not equal to $\Gamma_{\mathrm{c}}^{\mathrm{e}}$ for the FC-TFIM. At longer times, they also possess the same period as the order parameter and, in contrast to the regular cusps, their period increases with quench strength. Moreover, they separate less in time and are preceded by more smooth maxima in the return rate with decreasing quench strength.

However, it is to be emphasized that the distinctive signature of the anomalous phase is that its cusps are delayed in the sense that they always occur after the first minimum of the return rate. This leads to smooth peaks preceding them, with more such analytic peaks the smaller the quench is. This can be seen in Fig. 4, and agrees with what is observed in iMPS for the 

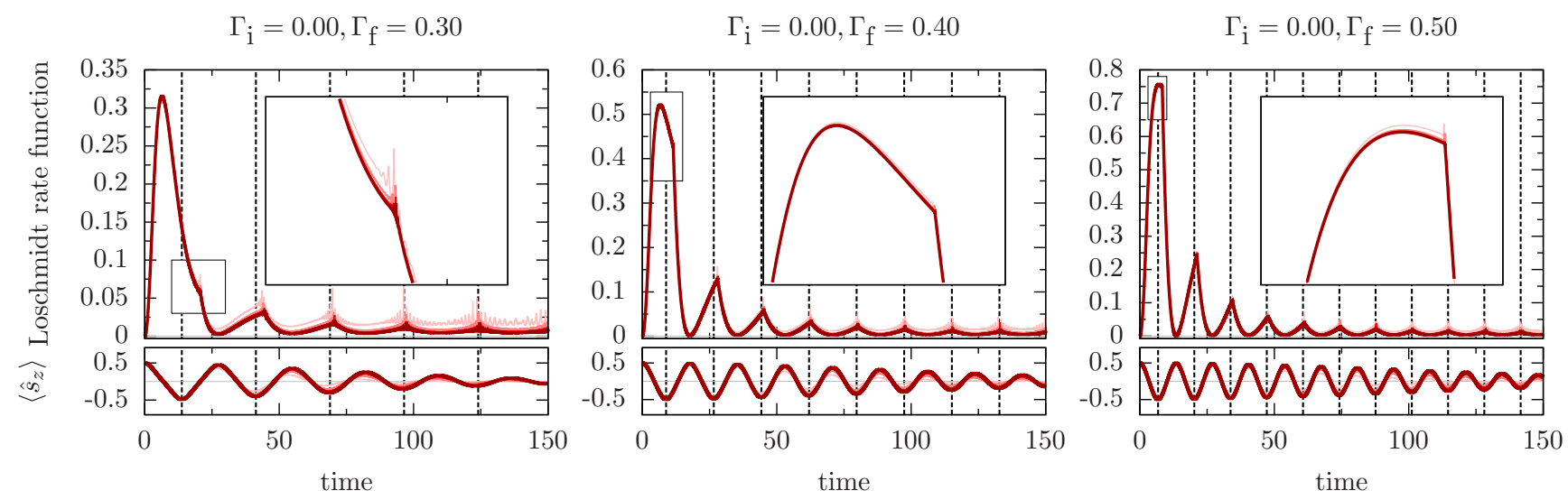

FIG. 3. Loschmidt-echo rate function and expectation value of the magnetization after a quench from $\Gamma_{\mathrm{i}}=0$ to $\Gamma_{\mathrm{f}}=0.30$ (left), $\Gamma_{\mathrm{f}}=0.40$ (middle), and $\Gamma_{\mathrm{f}}=0.50$ (right). All quenches are in the regular phase $\left(\Gamma_{\mathrm{f}}>\Gamma_{\mathrm{c}}^{\mathrm{d}}=0.25\right)$ (compare Fig. 4 for quenches in the anomalous phase). Each plot shows four different system sizes, $N=200,400,600,800$, from light to dark red, with the latter achieving convergence for the results shown here. The dotted grid indicates the turning points of $\left\langle s_{z}\right\rangle$ in the thermodynamic limit according to (8).

nonintegrable model [18] for $\alpha \lesssim 2.3$. Additionally, we find that the anomalous cusps occur for arbitrarily small quenches in the FC-TFIM.

The transition from the regular phase to the anomalous phase can be understood by observing the regular cusp before the first minimum of the return rate in each panel of Fig. 3. This cusp moves away from the first maximum and closer to the first minimum as $\Gamma_{\mathrm{f}}$ is decreased towards $\Gamma_{\mathrm{c}}^{\mathrm{d}}$. Once $\Gamma_{\mathrm{f}} \leqslant \Gamma_{\mathrm{c}}^{\mathrm{d}}$, this cusp crosses the first minimum of the return rate as we enter the anomalous phase (cf. Fig. 4). More details are provided in Appendix A.

It is evident in Figs. 3 and 4 that in the regular phase $\mathbb{Z}_{2}$ symmetry is preserved, whereas in the anomalous phase it is broken with a nonvanishing average of the order parameter, in agreement with the infinite-time limit of Fig. 2. This indicates that the DPT-I and DPT-II are intimately related by sharing a common critical point $\Gamma_{\mathrm{c}}^{\mathrm{d}}$. Also, Figs. 3 and 4 indicate that the period of the cusps in either dynamical phase and that of the oscillations of the order parameter are the same at long times. In fact, our simulations show that the period of the cusps also grows indefinitely as $\Gamma_{\mathrm{f}} \approx \Gamma_{\mathrm{c}}^{\mathrm{d}}$, in accordance with the diverging period of the order parameter shown in Fig. 2. As exemplified in Appendix B, all findings also hold for other initial conditions $\Gamma_{\mathrm{i}} \neq 0$.

Furthermore, we comment that unlike in the LR-TFIM for $\alpha \lesssim 2.3$ in Ref. [18], the Loschmidt-echo return rate in the case of the FC-TFIM does not exhibit double-cusp structures. We speculate that these double cusps may be related to the nonintegrability of the LR-TFIM, and would thus be missing in the case of the FC-TFIM. We leave this question open for future investigation.

Finally, we remark that our ED results were extensively tested for convergence on various environments and using different independent implementations. In cases where the Loschmidt echo is very small, i.e., for large system sizes and at times when the Loschmidt return rate is large, we observed that double-precision $(\approx 16$ significant digits $)$ ED is not sufficient to numerically resolve the Loschmidt return rate. In order to get
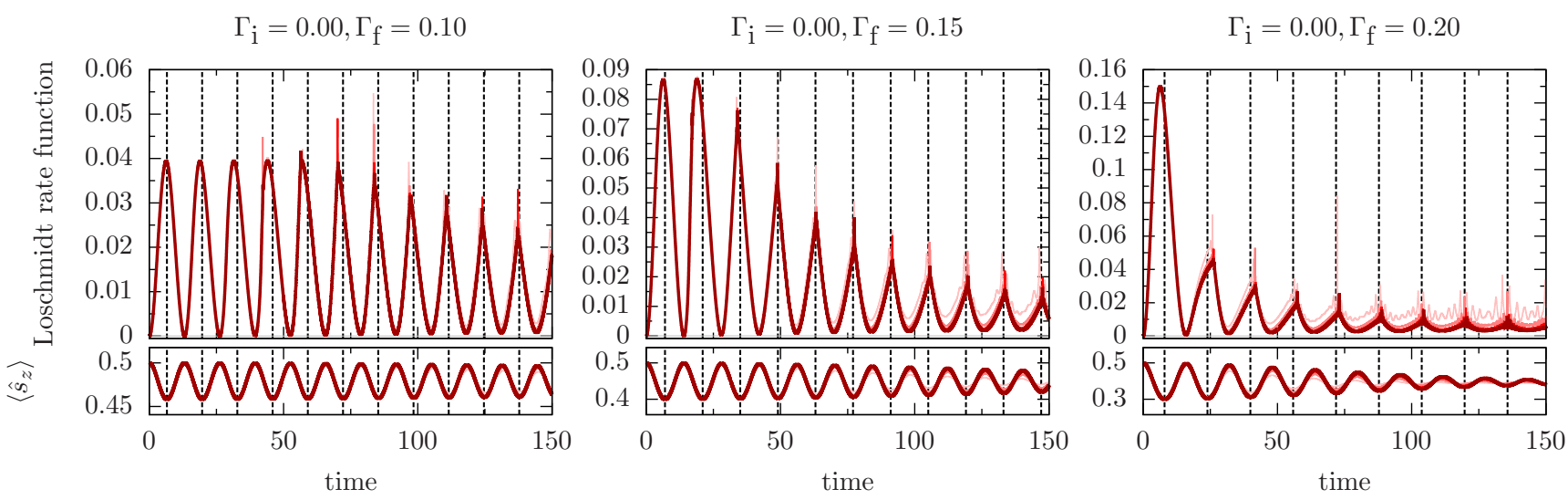

FIG. 4. Loschmidt-echo rate function and expectation value of the magnetization after a quench from $\Gamma_{\mathrm{i}}=0$ to $\Gamma_{\mathrm{f}}=0.10$ (left), $\Gamma_{\mathrm{f}}=0.15$ (middle), and $\Gamma_{\mathrm{f}}=0.20$ (right). All quenches are in the anomalous phase $\left(\Gamma_{\mathrm{f}}<\Gamma_{\mathrm{c}}^{\mathrm{d}}=0.25\right)$ (compare Fig. 3 for quenches in the regular phase). Each plot shows four different system sizes, $N=200,400,600,800$, from light to dark red, with the latter achieving convergence for the results shown here. The dotted grid indicates the turning points of $\left\langle s_{z}\right\rangle$ in the thermodynamic limit according to (8). 
rid of the numerical noise, we performed the numerical computations with enhanced precision of up to 256 significant digits.

\section{CONCLUSION}

Using semiclassical equations of motion and exact diagonalization, we have shown that the fully connected transverse-field Ising model exhibits two distinct dynamical phases, one of which seems to occur as a direct result of the long-range interactions in this model. Starting in a $\mathbb{Z}_{2}$-symmetry-broken ground state, quenches below the dynamical critical point give rise to the anomalous phase, whose defining signature is the occurrence of cusps only after the first minimum of the Loschmidt-echo return rate. On the other hand, quenches above the dynamical critical point lead to the regular phase, which shows cusps also before the first minimum of the return rate. The periods of the cusps in both phases display an intimate connection to the period of the $\mathbb{Z}_{2}$ order parameter oscillations. In fact, our ED simulations indicate that the anomalous phase coincides with the DPT-I phase of broken $\mathbb{Z}_{2}$ symmetry, while the regular phase with the DPT-I disordered phase. Our results agree with numerical results on the nonintegrable transverse-field Ising model with long-range interactions, obtained using an infinite matrix product state technique. Additionally, they provide support for the notion that long-range interactions bring about a new anomalous dynamical phase not found in short-range quantum spin chains. Our findings further extend the dynamical phase diagram of quantum spin chains with $\mathbb{Z}_{2}$ symmetry, and are suitable for investigation in ion-trap and optical cavity atom-photon experiments where interactions are long range.

\section{ACKNOWLEDGMENTS}

The authors acknowledge discussions with M. Heyl, S. Kehrein, and U. Schollwöck, and are grateful to M. Kastner for carefully reading and providing valuable comments on the manuscript. Financial support from the Deutsche Forschungsgemeinschaft (DFG) through Grant No. SFB/CRC1073 (Projects B03 and C03) is gratefully acknowledged. V.Z.-S. gratefully acknowledges support from the Austrian Science Fund (FWF): Grants No. F4104 SFB ViCoM and No. F4014 SFB FoQuS.

\section{APPENDIX A: TRANSITION FROM ANOMALOUS TO REGULAR PHASE}

As mentioned in the main text, the transition from the anomalous to the regular phase manifests in the presence of a cusp immediately preceding the first minimum of the return rate in time at $\Gamma_{\mathrm{f}} \gtrsim \Gamma_{\mathrm{c}}^{\mathrm{d}}$. This cusp then moves away from the first minimum to smaller times towards the first maximum of the return rate as one quenches deeper into the regular phase. Figure 5 shows this behavior in the vicinity of $\Gamma_{c}^{d}$. For quenches very close to, yet below $\Gamma_{\mathrm{c}}^{\mathrm{d}}$ (top panels of Fig. 5), the first cusp always appears after the first minimum of the return rate, which is the defining signature of the anomalous phase. However, for quenches right above $\Gamma_{\mathrm{c}}^{\mathrm{d}}$ (bottom panels of Fig. 5), we see that the first cusp is no longer preceded by a minimum in the return rate, which defines the regular phase. Also to be noted is that,
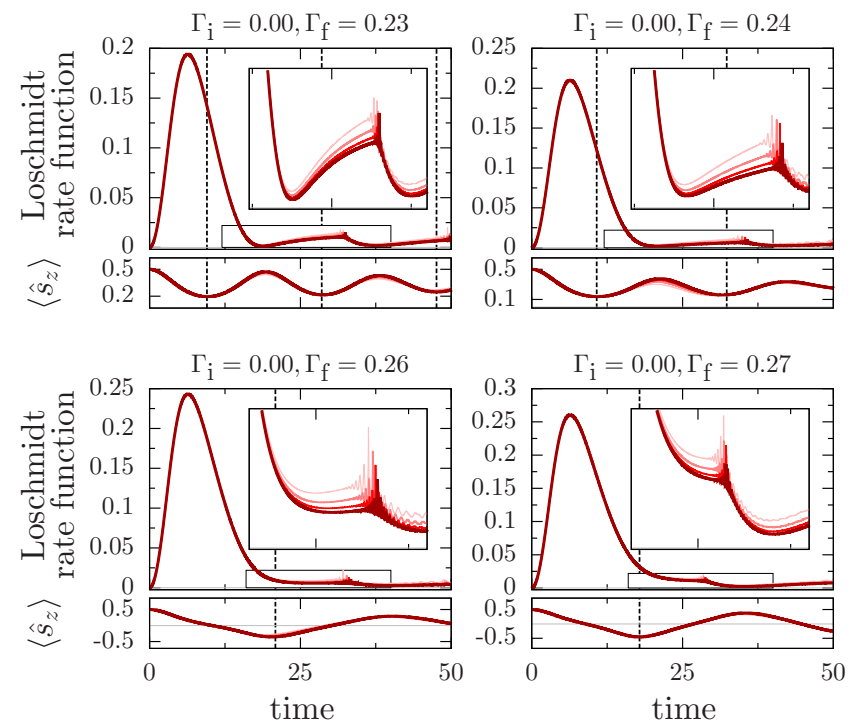

FIG. 5. Loschmidt-echo rate function and expectation value of the magnetization after a quench from $\Gamma_{\mathrm{i}}=0$ to $\Gamma_{\mathrm{f}}=0.23$ (top left), $\Gamma_{\mathrm{f}}=0.24$ (top right), $\Gamma_{\mathrm{f}}=0.26$ (bottom left), and $\Gamma_{\mathrm{f}}=0.27$ (bottom right). Quenches in the top (bottom) panels are in the anomalous (regular) phase $\Gamma_{\mathrm{f}}<\Gamma_{\mathrm{c}}^{\mathrm{d}}=0.25\left(\Gamma_{\mathrm{f}}>\Gamma_{\mathrm{c}}^{\mathrm{d}}=0.25\right)$. Each plot shows four different system sizes, $N=600,800,1000,1200$, from light to dark red. The dotted grid indicates the turning points of $\left\langle s_{z}\right\rangle$ in the thermodynamic limit according to (8).

in agreement with the main results of Figs. 3 and 4, Fig. 5 shows that the anomalous phase is linked to a finite nonzero average of the $\mathbb{Z}_{2}$ order parameter, while in the regular phase this order parameter vanishes.

Ideally, one would want to scan even closer to $\Gamma_{\mathrm{c}}^{\mathrm{d}}$, but this requires impracticable computational resources. The reason is that close to $\Gamma_{\mathrm{c}}^{\mathrm{d}}$, finite-size effects are particularly pronounced and one has to use large $N$ in order to see converged results. This can be understood from the semiclassical picture discussed in Sec. II B. For quenches close to $\Gamma_{c}^{d}$, the initial wave packet is localized near the homoclinic orbit of (5) (recall that for the quench to $\Gamma_{\mathrm{f}}=\Gamma_{\mathrm{c}}^{\mathrm{d}}$ the wave packet is exactly centered on the homoclinic orbit). As time evolves, the wave packet remains localized and follows the homoclinic orbit until it reaches the neighborhood of the unstable hyperbolic fixed point at $(s, p)=(0,0)$. Even though the wave packet is not centered exactly at the hyperbolic point, the wave packet's finite width of $\mathscr{O}(1 / \sqrt{N})$ makes it "feel" the unstable directions. As a consequence, the wave packet gets deformed and spreads in the unstable directions. This leads to a deviation from the $N \rightarrow \infty$ result where the width of the wave packet remains localized also close to the hyperbolic point. The closer one quenches to $\Gamma_{\mathrm{c}}^{\mathrm{d}}$, i.e., the closer the wave packet comes to the hyperbolic fixed point, the larger $N$ has to be to avoid these finite-size effects. Thus, even though for the main results of the paper $N=$ 800 leads to convergence, for the quenches in this appendix we have to go to larger $N$ to suppress most finite-size effects.

\section{APPENDIX B: QUENCHES FROM $\Gamma_{\mathbf{i}}=\mathbf{0 . 2 0}$}

We now look at the effect of changing the initial condition of our quench. Whereas the main part of the paper treats the case 

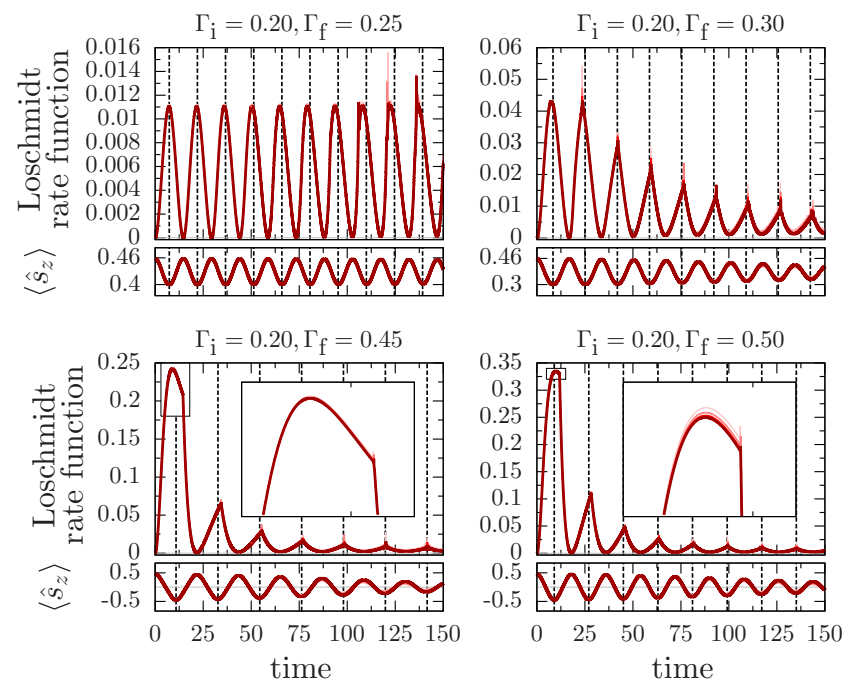

FIG. 6. Loschmidt-echo rate function and expectation value of the magnetization after a quench from $\Gamma_{\mathrm{i}}=0.20$ to $\Gamma_{\mathrm{f}}=0.25$ (top left), $\Gamma_{\mathrm{f}}=0.30$ (top right), $\Gamma_{\mathrm{f}}=0.45$ (bottom left), and $\Gamma_{\mathrm{f}}=0.50$ (bottom right). Quenches in the top (bottom) panels are in the anomalous (regular) phase $\Gamma_{\mathrm{f}}<\Gamma_{\mathrm{c}}^{\mathrm{d}}=0.35\left(\Gamma_{\mathrm{f}}>\Gamma_{\mathrm{c}}^{\mathrm{d}}=0.35\right)$. Each plot shows four different system sizes, $N=600,800,1000,1200$, from light to dark red, with the latter achieving convergence for the results shown here. The dotted grid indicates the turning points of $\left\langle s_{z}\right\rangle$ in the thermodynamic limit according to (8).
$\Gamma_{\mathrm{i}}=0$, quenches with different initial values of the transversefield strength lead to the same phase diagram with the only difference being quantitative because $\Gamma_{c}^{d}$ is a function of $\Gamma_{i}$ as expressed in (11). Nevertheless, the anomalous (regular) phase still manifests for quenches below (above) $\Gamma_{\mathrm{c}}^{\mathrm{d}}$. As an example, Fig. 6 shows four quenches from initial field strength $\Gamma_{\mathrm{i}}=0.20$. For this initial value of the transverse field, the dynamical critical point according to (11) is $\Gamma_{\mathrm{c}}^{\mathrm{d}}=0.35$ rather than 0.25 when $\Gamma_{\mathrm{i}}=0$ (see main results). In Fig. 6 we go from the anomalous phase (top panels) to the regular phase (bottom panels), where we see that in the anomalous phase the first cusp always occurs after the first minimum of the return rate, which is the defining feature of this phase. Note that the weaker the quench is in this phase, the more smooth maxima (and therefore the more smooth minima) precede the first cusp in time. However, after the transition to the regular phase, we see that the first cusp occurs before the first minimum of the return rate, which is the defining feature of this phase. This is qualitatively the same behavior as in the case of $\Gamma_{i}=0$ in the main part of the paper.

Additionally, Fig. 6 shows that the anomalous (regular) phase coincides with the $\mathbb{Z}_{2}$-symmetry-broken (unbroken) phase of the DPT-I for the case of $\Gamma_{\mathrm{i}}=0.20$. This is also in agreement with our results in Figs. 3, 4, and 5 for quenches from $\Gamma_{\mathrm{i}}=0$.
[1] B. Sciolla and G. Biroli, J. Stat. Mech. (2011) P11003.

[2] P. Smacchia, M. Knap, E. Demler, and A. Silva, Phys. Rev. B 91, 205136 (2015).

[3] B. Zunkovic, A. Silva, and M. Fabrizio, Philos. Trans. R. Soc. A 374, 20150160 (2016).

[4] B. Zunkovic, M. Heyl, M. Knap, and A. Silva, arXiv:1609.08482.

[5] J. C. Halimeh, V. Zauner-Stauber, I. P. McCulloch, I. de Vega, U. Schollwöck, and M. Kastner, Phys. Rev. B 95, 024302 (2017).

[6] M. Heyl, A. Polkovnikov, and S. Kehrein, Phys. Rev. Lett. 110, 135704 (2013).

[7] M. Heyl, Phys. Rev. Lett. 113, 205701 (2014).

[8] The nonanalyticities in the return rate would be phase transition points in the strict sense of the analogy to nonanalyticities in the thermal free energy at equilibrium. However, in this paper our characterization of a phase is determined by the kind of nonanalyticities (anomalous, regular, or none) that appear in the return rate.

[9] B. Pozsgay, J. Stat. Mech. (2013) P10028.

[10] L. Piroli, B. Pozsgay, and E. Vernier, J. Stat. Mech. (2017) 023106.

[11] J. M. Hickey, S. Genway, and J. P. Garrahan, Phys. Rev. B 89, 054301 (2014).

[12] S. Vajna and B. Dóra, Phys. Rev. B 89, 161105(R) (2014).

[13] S. Vajna and B. Dóra, Phys. Rev. B 91, 155127 (2015).

[14] M. Heyl, Phys. Rev. Lett. 115, 140602 (2015).

[15] M. Schmitt and S. Kehrein, Phys. Rev. B 92, 075114 (2015).

[16] M. Heyl, Phys. Rev. B 95, 060504 (2017).

[17] S. Campbell, Phys. Rev. B 94, 184403 (2016).
[18] J. C. Halimeh and V. Zauner-Stauber, arXiv:1610.02019.

[19] C. Karrasch and D. Schuricht, Phys. Rev. B 87, 195104 (2013).

[20] M. Fagotti, arXiv:1308.0277.

[21] E. Canovi, P. Werner, and M. Eckstein, Phys. Rev. Lett. 113, 265702 (2014).

[22] J. N. Kriel, C. Karrasch, and S. Kehrein, Phys. Rev. B 90, 125106 (2014).

[23] F. Andraschko and J. Sirker, Phys. Rev. B 89, 125120 (2014).

[24] S. Sharma, S. Suzuki, and A. Dutta, Phys. Rev. B 92, 104306 (2015).

[25] J. M. Zhang and H.-T. Yang, Europhys. Lett. 114, 60001 (2016).

[26] S. Sharma, U. Divakaran, A. Polkovnikov, and A. Dutta, Phys. Rev. B 93, 144306 (2016).

[27] N. O. Abeling and S. Kehrein, Phys. Rev. B 93, 104302 (2016).

[28] V. Stojevic et al., Phys. Rev. B 94, 165135 (2016).

[29] X. Peng et al., Phys. Rev. Lett. 114, 010601 (2015).

[30] N. Fläschner et al., arXiv:1608.05616.

[31] P. Jurcevic et al., Phys. Rev. Lett. 119, 080501 (2017).

[32] M. Kac, G. E. Uhlenbeck, and P. C. Hemmer, J. Math. Phys. 4, 216 (1963).

[33] M. Fannes, B. Nachtergaele, and R. Werner, Commun. Math. Phys. 144, 443 (1992).

[34] F. Verstraete, V. Murg, and J. I. Cirac, Adv. Phys. 57, 143 (2008).

[35] J. Haegeman et al., Phys. Rev. Lett. 107, 070601 (2011).

[36] J. Haegeman et al., Phys. Rev. B 94, 165116 (2016).

[37] V. Zauner-Stauber, L. Vanderstraeten, M. T. Fishman, F. Verstraete, and J. Haegeman, arXiv:1701.07035.

[38] S. R. White, Phys. Rev. Lett. 69, 2863 (1992).

[39] U. Schollwöck, Rev. Mod. Phys. 77, 259 (2005). 
[40] U. Schollwöck, Ann. Phys. (NY) 326, 96 (2011).

[41] S. R. White and A. E. Feiguin, Phys. Rev. Lett. 93, 076401 (2004).

[42] F. Verstraete, J. J. García-Ripoll, and J. I. Cirac, Phys. Rev. Lett. 93, 207204 (2004).

[43] G. Vidal, Phys. Rev. Lett. 93, 040502 (2004).
[44] A. J. Daley, C. Kollath, U. Schollwöck, and G. Vidal, J. Stat. Mech. (2004) P04005.

[45] D. Gobert, C. Kollath, U. Schollwöck, and G. Schütz, Phys. Rev. E 71, 036102 (2005).

[46] A. Das, K. Sengupta, D. Sen, and B. K. Chakrabarti, Phys. Rev. B 74, 144423 (2006). 


\section{Part III}

Entanglement and time 



\title{
5 History state entanglement
}

\begin{abstract}
"These two ways of thinking, the way of time and history and the way of eternity and of timelessness, are both part of man's effort to comprehend the world in which he lives. Neither is comprehended in the other nor reducible to it. They are, as we have learned to say in physics, complementary views, each supplementing the other, neither telling the whole story."
\end{abstract}

J. Robert Oppenheimer, Science And The Common Understanding

\begin{abstract}
"The physical universe was a language with a perfectly ambiguous grammar. Every physical event was an utterance that could be parsed in two entirely different ways, one causal and the other teleological, both valid, neither one disqualifiable no matter how much context was available."
\end{abstract}

Ted Chiang, Stories of Your Life and Others

In this chapter we use the Page-Wootters construction 177 of time independent history states, and ideas of quantum information theory to classify the unitary Scrödinger dynamics in closed quantum systems. A history state is element in an extended Hilbert space, the tensor product of the system Hilbert space and a time-like Hilbert space. Projections of the history state onto definite time states yield the system state at the respective instant of time. As such, the history state is used to encode the evolution of the system within a given time window of observation. We investigate entanglement in the history state w.r.t. a bipartition into system and time. More precisely, we study how bipartite entanglement entropies grow as a function of observation time. This constitutes a global, operator-independent characterization of the system dynamics encoded in the history state. An artistic illustration ${ }^{1}$ is given in Figs. 5.1 and 5.2 .

The von Neumann entropy, and the second Rényi (collision) entropy, are of particular interest. First, the von Neumann entropy has an instructive interpretation facilitated by Holevo's bound [181] as the precision of an internal quantum clock. Fundamental quantum speed limits [182, 183] bound the precision of this clock. Second, the second Rényi entropy is a function of two point correlations in the local density of states. Consequently, for large observation times, the collision entropy can be used to detect level repulsion, a key quantity to discriminate between integrable and non-integrable systems.

After introducing the formalism in more details in Secs. 5.1 and 5.2, we elaborate on the information theoretic interpretation in Sec. 5.3. Thereafter, two concrete examples are discussed. First, a random matrix ensemble of Hermitian Wigner matrices in Sec. 5.4. And, second, a real

\footnotetext{
${ }^{1}$ A picture is worth a thousand words. The mental image of Schrödinger's cat 178 as an illustration of superposition of quantum states and entanglement has gained popularity beyond the sciences to the general public. Arnold's cat map 179 is a famous chaotic discrete dynamical system with positive Kolmogorov entropy. In 180 Jaynes uses a cat to make a point about the anthropomorphic nature of (thermodynamic) entropy. Therefore, the author feels encouraged to borrow the cat as a pictorial illustration of the results in the context of both entanglement, non-integrability, as well as entropy.
} 
symmetric hardcore boson lattice mode $2^{2}$ with GOE level statistics in the non-integrable regime, cf. Sec. 5.5.

\subsection{Extended Hilbert space and history states}

Page-Wootters formalism. The idea to extend the system Hilbert space $\mathcal{H}_{S}$, in which the physical state

$$
\left|\psi_{t}\right\rangle=e^{-i H t}\left|\psi_{0}\right\rangle
$$

evolves according to the Hamiltonian $H$, to a larger Hilbert space $\mathcal{H}_{e}=\mathcal{H}_{S} \otimes \mathcal{H}_{T}$, in which the family of time evolved states is a single time-independent state, goes back to Page and Wootters [177], and was further developed in [184]. Page and Wootters pursued a single quantum mechanical description of observer and system, in which the time dependence of observed quantities is a consequence of an internal quantum mechanical clock without external time parameter.

In a more recent formulation 184 of the Page Wootters mechanism, the time-like Hilbert space $\mathcal{H}_{T}=L^{2}(\mathbb{R})$ is equipped with a time operator $T$ and its conjugate $\Omega$ such that $[T, \Omega]=i$. For any $|\Psi\rangle \in \mathcal{H}_{e}$ the physical state at time $t$ is then obtained by projecting onto a (generalized) time operator eigenstate $|t\rangle$ with eigenvalue $t$, i.e. $|\psi(t)\rangle=\langle t \mid \Psi\rangle$. The family of states $|\psi(t)\rangle$ obtained in this way, comes from the dynamics of the Schrödinger Eq. (5.1) if, and only if, $|\Psi\rangle$ obeys the Wheeler-DeWitt type equation $\left(1_{S} \otimes \Omega+H_{S} \otimes 1_{T}\right)|\Psi\rangle=0$ [184]. The last Eq. can therefore be written more explicitly as

$$
|\Psi\rangle=\int d t e^{-i H t}\left|\psi_{0}\right\rangle|t\rangle_{T}
$$

Note that the norm of $|\Psi\rangle$ is fixed by the requirement that $\langle t \mid \Psi\rangle=|\psi(t)\rangle$ is normalized. In particular, $|\Psi\rangle$ is not normalized in $\mathcal{H}_{S} \otimes \mathcal{H}_{T}$, but its 'marginals' $\langle t \mid \Psi\rangle$ are normalized in $\mathcal{H}_{S}$ for all $t$.

In addition, extended Hilbert spaces with a finite dimensional time Hilbert space $\mathcal{H}_{T}=\operatorname{span}\{|t\rangle$ : $t \in \mathcal{T}\}$, for some finite set $\mathcal{T}=\left\{t_{1}, \cdots, t_{|\mathcal{T}|}\right\} \subset \mathbb{R}$ of times have been considered 185. By definition, the states $|t\rangle$ are orthonormal, i.e. $\left\langle t^{\prime} \mid t\right\rangle=\delta_{t^{\prime}, t}$, and serve as ancillary time labels. In contrast to the infinite dimensional case of $\mathcal{H}_{T}=L^{2}(\mathbb{R})$ above, it is not possible to define operators $T$ and $\Omega$ that fulfill the canonical commutation relations on any finite dimensional Hilbert space. As we are ultimately interested in entanglement entropies of pure states $|\Psi\rangle$ in $\mathcal{H}_{e}$, we consider normalized states

$$
|\Psi\rangle=\frac{1}{\sqrt{|\mathcal{T}|}} \sum_{t \in \mathcal{T}} e^{-i H t}\left|\psi_{0}\right\rangle|t\rangle .
$$

We do not impose a Wheeler-DeWitt type equation for this state. Still, projections $\langle t \mid \Psi\rangle=$ $\left|\psi_{t}\right\rangle / \sqrt{|\mathcal{T}|}$ of this state onto definite time states are proportional to the time-evolved system state $\left|\psi_{t}\right\rangle$. One cannot expect that the projections are equal to the state $\left|\psi_{t}\right\rangle$, due to the different normalization compared to (5.2). Equation (5.3) is the finite time sample analog of (5.2). The times are sampled uniformly from the set $\mathcal{T}$. More generally, we also consider history states

$$
|\Psi\rangle=\sum_{t \in \mathcal{T}} \sqrt{p_{t}} e^{-i H t}\left|\psi_{0}\right\rangle|t\rangle,
$$

in which the times $t \in \mathcal{T}$ are sampled from a probability distribution $p_{t}$.

We generalize the state (5.4) to the situation of the infinite dimensional time-like Hilbert space

\footnotetext{
${ }^{2}$ The spectrum of this model has been computed and kindly provided by Nils Abeling.
} 
$\mathcal{H}_{T}=L^{2}(\mathbb{R})$,

$$
|\Psi\rangle=\int_{\mathbb{R}} d t \sqrt{\Phi(t)} e^{-i t H}\left|\psi_{0}\right\rangle|t\rangle_{T}
$$

where $\sqrt{\Phi}: \mathbb{R} \rightarrow \mathbb{C}$ is a normalized ${ }^{3} L^{2}$ function, i.e. $\int|\Phi|=1$, and $|t\rangle$ is a (generalized) orthonormal basis of $\mathcal{H}_{T}$, i.e. $\left\langle t^{\prime} \mid t\right\rangle=\delta\left(t-t^{\prime}\right)$. The function $\Phi(t)$ is the continuous analog of the discrete probability masses $p_{t}$. This state does not obey the Wheeler-DeWitt equation, either, and $\langle t \mid \Psi\rangle$ is equal to $\sqrt{\Phi(t)} e^{-i t H}\left|\psi_{0}\right\rangle$. The normalization of (5.5) forces us to sacrifice the simple relation $\langle t \mid \Psi\rangle=|\psi(t)\rangle$. Moreover, we do not require the existence of a time operator $T$ on $\mathcal{H}_{T}$, whose generalized eigenstates are $|t\rangle$. Instead, it suffices to view $|t\rangle$ as ancillary states, which serve as a bookkeeping device and label different states $|\psi(t)\rangle$ in $|\Psi\rangle$.

The non-normalizable state (5.2) is obtained for the non-normalizable uniform function $\Phi(t)=$ 1 , in (5.5). Furthermore, the states (5.3) and (5.4) are formally obtained by using $\sqrt{\Phi(t)}=$ $\frac{1}{|\mathcal{T}|} \sum_{t^{\prime} \in \mathcal{T}} \delta\left(t-t^{\prime}\right)$ and $\sqrt{\Phi(t)}=\sum_{t^{\prime} \in \mathcal{T}} \sqrt{p_{t^{\prime}}} \delta\left(t-t^{\prime}\right)$ in $(5.5)$, respectively. We refer to the states (5.3), (5.4) and 5.5) as (Feynman-Kitaev) history states [184, 186]. The function $\Phi$ can be viewed as a time window, which specifies the effective time of observation. In particular, when $\Phi$ has compact support, the history of the state is only taken into account for times in the support of $\Phi$. Intuitively, for non-compact support, one can identify the effective time of observation with those times for which $\Phi$ is non-negligible.

In the sequel we mainly consider the uniform function

$$
\Phi_{\text {box }}(t)=1_{[-1 / 2,1 / 2]}(t) .
$$

Another useful choice is

$$
\Phi_{\text {Gauss }}(t)=(2 \pi)^{-1 / 2} \exp \left(-t^{2} / 2\right)
$$

Remark (time dilation). For any normalized $\sqrt{\Phi} \in L^{2}(\mathbb{R})$, and $c>0$, its (normalized) dilation is defined as $\Phi_{c}(t)=\Phi(t / c) / c$. One may think of $\Phi$ as a function on scale $t \sim \mathcal{O}(1)$ and $\Phi_{c}$ as a function on scale $t \sim \mathcal{O}(c)$. Hence, $c$ is a scaling parameter of dimension time. This scaling parameter can also be absorbed into the system Hamiltonian $H$. By a change of variables $t / c \mapsto t$,

$$
\left|\Psi_{c}\right\rangle=\int d t \sqrt{\Phi_{c}(t)}\left|\psi_{t}\right\rangle|t\rangle=\sqrt{c} \int d t \sqrt{\Phi(t)} e^{-i c t H}\left|\psi_{0}\right\rangle|c t\rangle
$$

(note that $c\left\langle c t \mid c t^{\prime}\right\rangle=\left\langle t \mid t^{\prime}\right\rangle$ ). Hence, the rescaling of time $t \mapsto c t$ is equivalent to a rescaling $E \mapsto E / c$ of energies. This shows the conjugate relation between time and energy on which the interpretation of the subsequent results is based.

Remark (time translation). For a translated window of observation $\Phi(t) \mapsto \Phi\left(t-t_{0}\right)$ the history state changes according to

$$
|\Psi\rangle \mapsto \int d t \sqrt{\Phi(t)}\left|\psi_{t+t_{0}}\right\rangle\left|t+t_{0}\right\rangle
$$

Remark (time modulation). Modulations of the time window function $\Phi(t) \mapsto \Phi(t) e^{-2 i t E}$ for some fixed $E \in \mathbb{R}$ yield a shift of the spectrum of $H$,

$$
|\Psi\rangle \mapsto \int d t \sqrt{\Phi(t)} e^{i t E}\left|\psi_{t}\right\rangle\left|t+t_{0}\right\rangle=\int d t \sqrt{\Phi(t)} e^{-i t\left(H_{S}+E\right)}\left|\psi_{0}\right\rangle|t\rangle
$$

\footnotetext{
${ }^{3}$ In contrast to [184, normalization in the extended Hilbert space is important, as we are going to compute entanglement properties of $|\Psi\rangle$ by means of entanglement entropies.
} 


\subsection{History state entanglement entropy}

\subsubsection{Reduced history matrix}

To compute the (Rényi) entanglement entropy of the state 5.5 w.r.t. the bipartition $\mathcal{H}_{S} \otimes \mathcal{H}_{T}$ into system and time, we need the reduced density matrix

$$
\rho_{c}=\operatorname{Tr}_{T}\left|\Psi_{c}\right\rangle\left\langle\Psi_{c}\left|=\int d t\right| \Phi(t)|| \psi_{c t}\right\rangle\left\langle\psi_{c t}\left|=\int d t\right| \Phi_{c}(t)|| \psi_{t}\right\rangle\left\langle\psi_{t}\right|
$$

This is an incoherent superposition of time evolved states, and we refer to $\rho_{c}$ as the reduced history matrix or simply history matrix. The matrix $\rho_{c}$ is normalized, $\operatorname{Tr}_{S} \rho_{c}=\int d t|\Phi(t)|^{2}=1$, because $\left|\psi_{c t}\right\rangle$ is normalized. More explicitly, in the energy eigenbasis of $H$ the reduced density matrix reads

$$
\left(\rho_{c}\right)_{i j}=\int d t|\Phi(t)| e^{-i c t\left(E_{i}-E_{j}\right)}\left\langle E_{i} \mid \psi_{0}\right\rangle\left\langle\psi_{0} \mid E_{j}\right\rangle .
$$

Remark (time translation). Under a translation of time $t \mapsto t+t_{0}$, cf. Eq. (5.8), the reduced density matrix changes according to

$$
\left(\rho_{c}\right)_{i j} \mapsto\left(\rho_{c}\right)_{i j} e^{-i c t_{0}\left(E_{i}-E_{j}\right)} .
$$

Time translation leads to a phase modulation as a function of energy in the history matrix. This is an instance of the fact that translation translates to phase modulation under Fourier transform. Essentially, the quantum mechanical time evolution of a state is a Fourier transform in the energy eigenbasis, and the eigenenergies are the Fourier frequencies.

Remark (time modulation invariance). As the reduced history matrix depends on the spectrum only through energy differences, it is invariant under time modulation (5.9). Equivalently, $\rho_{c}$ depends on the time window function only through its modulus $|\Phi|$. Hence, regarding quantities that depend only on the reduced state $\rho_{c}$, one may, without loss of generality, assume that $\Phi$ is real-valued. Nevertheless, a modulation of the history state can be useful when subsequent approximations of the reduced density matrix break the modulation invariance. More specifically, when the initial state $\left|\psi_{0}\right\rangle$ has a spectral density concentrated around the expected energy $E_{0}$, modulation by $e^{i E_{0} t}$, equivalently, shifting the spectrum by $E_{0}$, and an expansion in powers of $H$ results in an expansion of (decaying) centered moments.

\subsubsection{Rényi Entropies}

We refer to the $k$ th Rényi entropy $S_{k}(c)=\frac{1}{1-k} \log \operatorname{Tr}_{S} \rho_{c}^{k}$ of the history matrix $\rho_{c}$ as the history state entanglement entropy. To compute the trace of the $k$ th power of $\rho_{c}$, we assume a finite dimensional Hilbert space $\mathcal{H}_{S}$, and expand the initial state in the orthonormal energy eigenbasis $\left|E_{i}\right\rangle$ (possible energy degeneracies are accounted for by allowing $E_{i}=E_{j}$ for $i \neq j$ ) with $E_{i} \in \sigma(H)$. 
Thus, identifying $t_{k+1}=t_{1}$ and $t_{0}=t_{k}$ to abbreviate notations,

$$
\begin{aligned}
\operatorname{Tr} \rho_{c}^{k} & =\int d^{k} t \prod_{j=1}^{k}\left|\Phi_{c}\left(t_{j}\right)\right|\left\langle\psi\left(t_{j}\right) \mid \psi\left(t_{j+1}\right)\right\rangle \\
& =\sum_{E_{1}, \cdots E_{k} \in \sigma(H)} \int d^{k} t \prod_{j=1}^{k}\left|\Phi\left(t_{j}\right)\right|\left|\left\langle\psi_{0} \mid E_{j}\right\rangle\right|^{2} e^{-i c\left(t_{j+1}-t_{j}\right) E_{j}} \\
& =\sum_{E_{1}, \cdots E_{k} \in \sigma(H)} \prod_{j=1}^{k}\left|\left\langle\psi_{0} \mid E_{j}\right\rangle\right|^{2}\left[\int d t|\Phi(t)| e^{-i c t\left(E_{j-1}-E_{j}\right)}\right] \\
& =\sum_{E_{1}, \cdots E_{k} \in \sigma(H)} \prod_{j=1}^{k}\left|\left\langle\psi_{0} \mid E_{j}\right\rangle\right|^{2} \widetilde{\Phi}\left(c E_{j-1}-c E_{j}\right),
\end{aligned}
$$

where $\widetilde{\Phi}(E)=\int|\Phi| e^{-i t E} d t$ is the Fourier transform of $|\Phi|$. For the examples of (5.6), one has

$$
\begin{aligned}
\widetilde{\Phi}_{\text {box }}(E) & =\operatorname{sinc}(E / 2) \\
\widetilde{\Phi}_{\text {Gauss }}(E) & =\exp \left(-E^{2} / 2\right)
\end{aligned}
$$

In particular, for symmetric $\widetilde{\Phi}$, the second Rényi (collision) entropy reads

$$
S_{2}(c)=-\log \left[\sum_{E_{1}, E_{2} \in \sigma(H)}\left|\left\langle\psi_{0} \mid E_{1}\right\rangle\right|^{2}\left|\left\langle\psi_{0} \mid E_{2}\right\rangle\right|^{2} \widetilde{\Phi}\left(c E_{1}-c E_{2}\right)^{2}\right] .
$$

Remark (time translation invariance). The transformation $(\rho)_{i j} \mapsto(\rho)_{i j} e^{-i\left(E_{i}-E_{j}\right) t_{0}}$ of the reduced history matrix under time translation, cf. Eq. (5.10), results into time translation invariance of the entanglement entropy. This can be seen as follows. The trace of any monomial, $\operatorname{Tr} \rho^{n}=\rho_{i_{1} i_{2}} \rho_{i_{2} i_{3}} \cdots \rho_{i_{n} i_{1}}$, of $\rho$ is apparently invariant under this transformation. Using this fact in the expansion of any function $f(\rho)$, in particular, for $f(x)=-x \log x$, and the linearity of the trace, yields the invariance of $\operatorname{Tr} f(\rho)$.

Remark (phase invariance). All Rényi entropies depend only on the initial system state $\left|\psi_{0}\right\rangle$ through the modulus $\left|\left\langle\psi_{0} \mid E\right\rangle\right|$ of the overlaps with the energy eigenstates.

\subsubsection{Summary}

An extended Hilbert space is defined by tensoring a time-like Hilbert space to the system Hilbert space, on which the time evolution due to Schrödinger's equation is defined. The idea goes back to Page and Wootters [177]. The time-like Hilbert space can be finite and infinite dimensional for discrete and continuous time sampling. Expanding times in the unit interval in binary representation up to accuracy $2^{-T}$ naturally yields a $2^{T}$ dimensional time-like Hilbert space for which time instants may be interpreted as configurations of time spin. We have reviewed the notion of history states, a class of states in the extended Hilbert space, which are derived from the unitary Schrödinger evolution. Our notion differs from the history states considered in [184] in respect of the normalization. To compute meaningful entanglement entropies, our history states need to be normalized, which prevents a Wheeler-DeWitt interpretation. For infinite dimensional time-like Hilbert space $\mathcal{H}_{T}=L^{2}(\mathbb{R})$, the history states are normalized by introducing the $L^{2}(\mathbb{R})$ normalized function $\Phi$. The (effective) support of $\Phi$ can be interpreted as the (effective) observation time. 


\section{History state entanglement}

Phase modulations of $\Phi$ can be absorbed by shifting the spectrum. Dilations of $\Phi$, i.e. rescaling of the observation time, is equivalent to rescaling of the spectrum. Translation of $\Phi$ leads to phase modulations of the reduced density matrix in the energy eigenbasis. These relations are summarized in Table 5.1.

The entanglement entropy of a history state w.r.t. a bipartition into time-like Hilbert space and system Hilbert space is invariant under modulation and translation of $\Phi$, but not invariant under time dilation, cf. table 5.1. In other words, the entanglement entropy of a history state is independent of when the observation begins, but it depends on the duration of observation. Even though modulation, translation and dilation do not commute, the invariance of two of those guarantees that one only needs to consider dilations. We denote the dilation parameter by $c$, and, henceforth, consider the entanglement entropy as a function of $c$. We consider time window functions with (effective) support of size $\mathcal{O}(1)$, such that the dilated time window has (effective) support on $\mathcal{O}(c)$ and $c$ is the effective time of observation.

\begin{tabular}{l|ccc} 
time window & $\begin{array}{c}\text { translation, } \\
\Phi(t) \mapsto \Phi\left(t-t_{0}\right)\end{array}$ & $\begin{array}{c}\text { modulation, } \\
\Phi(t) \mapsto \Phi(t) e^{i t E}\end{array}$ & $\begin{array}{c}\text { dilation, } \\
\Phi(t) \mapsto \Phi(t / c) / \sqrt{c}\end{array}$ \\
\hline \hline history state & Eq. (5.8) & Eq. (5.9) & Eq. (5.7) \\
\hline history matrix & Eq. (5.10) & invariant & not invariant \\
\hline $\begin{array}{l}\text { entanglement } \\
\text { entropy }\end{array}$ & invariant & invariant & not invariant
\end{tabular}

Table 5.1: The effect of translation, modulation, and dilation of the time window function $\Phi$ on the history state, the history matrix, and the entropy of the history matrix.

The entanglement entropy depends on the initial state $\psi_{0}$ only through the modulus of the overlaps with the energy eigenstates. More specifically, the entanglement entropy is a function of the local partition function $\sum_{i}\left|\left\langle\psi_{0} \mid E_{i}\right\rangle\right|^{2} \delta\left(E-E_{i}\right)$. As a function of $c$, the entanglement entropy constitutes a global, operator-independent characterization of the closed quantum dynamics underlying the history state. Before we give two examples of this in Secs. 5.4 and 5.5, we elaborate more on the (information theoretic) significance of the history entanglement entropy.

\subsection{Interpretation of the history entanglement}

In the first contact science fiction novel "Story of your life" by Ted Chiang, scientists try to decode the language of some extraterrestrial life form. The life form is called heptapods, because they have seven limbs. As it turns out, the language and the way of thinking of the heptapods is quite different from humans. In contrast to humans, heptapods do not perceive time sequentially, and hence do not have a clear distinction between past, present, and future. This is reflected in their spoken language and their two-dimensional diagrammatic written language. As a consequence, for the heptapods Maupertuis's variational principal is more elementary than for humans, and their technology has developed differently (on a superior level compared to human technology). One may imagine that the concept of extended Hilbert spaces and history states of the form (5.5) are natural from a heptapod's perspective. Coming back to the sequential interpretation of time, the question is 'what can one learn from the history states from a anthropological perspective?'. We give two answers to this question. First, we elaborate on the implications for measurements of operators on the system Hilbert space in the following Sec. 5.3.1. Second, thereafter, in Sec. 5.3.2. 
an information theoretic interpretation of von Neumann entanglement entropy based on Holevo's bound is given.

\subsubsection{Operators and measurement on $\mathcal{H}_{e}$}

Eventually, one is interested in expectation values $\left\langle O_{S}(t)\right\rangle$, and correlations $\left\langle O_{S}^{(1)}\left(t_{1}\right) O_{S}^{(2)}\left(t_{2}\right)\right\rangle$, etc., of operators $O_{S}$ and $O_{S}^{(1,2)}$ on $\mathcal{H}_{S}$ in the physical state $|\psi(t)\rangle$. The history state $|\Psi\rangle$ and operators on $\mathcal{H}_{e}$ have no a priori physical interpretation but should rather be thought of as a formal ancillary construction. The usefulness of $|\Psi\rangle$ comes from the observation that the physical expectation values can be written as 'ancillary expectation values' of operators on the extended Hilbert space in this state, e.g. $\left\langle O_{S}(t)\right\rangle=\left\langle\Psi\left|O_{e}\right| \Psi\right\rangle$, with $O_{e}=|t\rangle O_{S}\langle t|$.

Entanglement of measurement in general bipartite systems. We look at expectation values

$$
\langle\Psi|O| \Psi\rangle
$$

of an observable $O$ on a generic bipartite Hilbert space $\mathcal{H}=\mathcal{H}_{A} \otimes \mathcal{H}_{B}$ more closely. Assuming the operator $O=O_{A} \otimes \mathbf{1}_{B}$ acts as the identity on one factor of $\mathcal{H}$, say $\mathcal{H}_{B}$, then (5.14) simplifies to

$$
\langle\Psi|O| \Psi\rangle=\operatorname{Tr}_{A}\left(\rho_{A} O_{A}\right)
$$

where $\rho_{A}=\operatorname{Tr}_{B}|\Psi\rangle\langle\Psi|$. The fact that $\rho_{A}$ may not be of rank one (i.e. when $|\Psi\rangle$ is entangled), comes from quantum correlations between $\mathcal{H}_{A}$ and $\mathcal{H}_{B}$ in $|\Psi\rangle$. More precisely, $|\Psi\rangle$ is entangled (w.r.t. the splitting $\mathcal{H}_{A} \otimes \mathcal{H}_{B}$ ) if, and only if, there are operators $O_{1}=O_{A} \otimes \mathbf{1}_{B}$ and $O_{2}=\mathbf{1}_{A} \otimes O_{B}$, such that $\left\langle O_{1} O_{2}\right\rangle-\left\langle O_{1}\right\rangle\left\langle O_{2}\right\rangle \neq 0$. Knowing only $\rho_{A}$, but not $|\Psi\rangle$, means one cannot recover non-vanishing correlations of the form $\left\langle O_{1} O_{2}\right\rangle_{c}$. This information is stored non-locally between subsystems $A$ and $B$ in the form of entanglement.

A quantitative formulation is provided by the von Neumann entanglement entropy of measurement, $S_{\mathrm{vN}}\left(\rho_{A}\right)$.

Given an observable $O_{A}=\sum_{i} \lambda_{i}\left|\lambda_{i}\right\rangle\left\langle\lambda_{i}\right|$ on $\mathcal{H}_{A}$ with non-degenerate eigenvalues $\lambda_{i}$ and the associated probabilities $p_{i}=\left\langle\lambda_{i}\left|\rho_{A}\right| \lambda_{i}\right\rangle$ to measure $\lambda_{i}$ in the mixed state $\rho_{A}$, then $S_{\mathrm{vN}}\left(\rho_{A}\right) \leq H\left(p_{i}\right)$, with equality if $O_{A}$ and $\rho_{A}$ commute.

On the one hand, the expectation value of many repeated measurements of $O_{A}$ is given by (5.15). On the other hand, the Shannon entropy of the distribution of individual measurement outcomes is bounded from below by $S_{\mathrm{vN}}\left(\rho_{A}\right)$. In other words, the average information gain (surprise) of a non-trivial observable cannot be reduced indefinitely if the state is entangled.

Consider two cases. First, assume that $|\Psi\rangle$ is not entangled, and $\rho_{A}$ is pure. Then, there exists a non-trivial observable with non-degenerate eigenvalues that yields a definite measurement outcome. Any observable with an eigenspace spanned by the one-dimensional support of $\rho_{A}$ will do. Second, when $|\Psi\rangle$ is entangled, such that $\rho_{A}$ has rank larger than one, any observable with non-degenerate eigenspaces must yield positive (Shannon) information $H$ of at least $S\left(\rho_{A}\right)$ per measurement on average. The fluctuations of individual measurement outcomes cannot be reduced indefinitely as a consequence of entanglement. The fluctuations of the measurement outcomes is minimal for observables that are diagonal in the basis that diagonalizes $\rho_{A}$. But no non-trivial local observable on $\mathcal{H}_{A}$ with definitive measurement outcome exists if $|\Psi\rangle$ is entangled. The remaining surprise comes from non-locally stored information between subsystems $A$ and $B$ in the form of entanglement. 
Measurements on $\mathcal{H}_{S}$. Now, we consider the bipartition of the extended Hilbert space $\mathcal{H}_{e}$ into $\mathcal{H}_{A}=\mathcal{H}_{S}$ and $\mathcal{H}_{B}=\mathcal{H}_{T}$. For the sake of concreteness, we consider the history state (5.4) with discrete times, but the discussion applies to the continuous case of Eq. (5.5) as well. Then,

$$
\left\langle\Psi\left|O_{S}\right| \Psi\right\rangle=\sum_{t} p_{t}\left\langle\psi(t)\left|O_{S}\right| \psi(t)\right\rangle
$$

and the reduced density matrix $\rho=\sum_{t} p_{t}|\psi(t)\rangle\langle\psi(t)|$ is an incoherent superposition of the evolved state at times in $\mathcal{T}$. Expectation values of operators $O_{S} \otimes \mathbf{1}_{T}$ in $|\Psi\rangle$ cannot resolve the time dynamics of $|\psi(t)\rangle$, and only yield weighted time averages of the form (5.16). One cannot obtain the expectation value $\left\langle\psi(t)\left|O_{S}\right| \psi(t)\right\rangle$ at a specific time $t$ by measuring the state $\rho$. Metaphorically speaking, the measuring device draws a time-evolved state at a random instant of time (from the ensemble given by $\rho$ ), measures the observable $O_{S}$ in that state and returns the result. From an practical point of view, one may picture measurements in the reduced state $\rho$ as a mean to model unavoidable variations of the measurement instant in repeated experiments.

No information at which time $t$ the system is in the state $|\psi(t)\rangle$ is contained in $\rho$. In the limiting case, when $|\psi(t)\rangle$ is constant in time, the reduced density matrix $\rho$ has rank one (no information is lost), and one can recover everything about the states $|\psi(t)\rangle$ by measuring observables of the type $O_{S} \otimes \mathbf{1}_{T}$. In particular, observables $O_{S}$ with an eigenspace spanned by $|\psi(0)\rangle$ yield definite measurement outcomes with zero Shannon information. Those observables determine $|\psi(t)\rangle$ with perfect fidelity. In contrast, when $|\psi(t)\rangle$ is non-constant in time, the measurement of any non-trivial observable of the form $O_{S} \otimes \mathbf{1}_{T}$ in $|\Psi\rangle$ must yield positive (Shannon) information. The distribution of individual measurement outcomes in the time average $(5.16)$ has a minimal Shannon entropy of $S_{\mathrm{vN}}(\rho)$, which cannot be reduced. It is a consequence of the fact that the information at which time $t$ the system is in its state $|\psi(t)\rangle$ is not contained in $\rho$.

The von Neumann entropy of $\rho$ is a measure of how well $|\psi(t)\rangle$ explores the Hilbert space $\mathcal{H}_{S}$. The following section also alludes to this interpretation. More precisely, by Holevo's theorem, the von Neumann entropy $S(\rho)$ is an upper bound on the amount of accessible information about the instant of time $t \in \mathcal{T}$ obtainable through measurements on the mixed state $\rho$.

\subsubsection{Holevo's bound and quantum clocks}

One main difference between classical and quantum information theory comes from the fact that different classical states are distinguishable, while different (pure) quantum states can only be distinguished with certainty if they are orthogonal. This underlies both, the non-cloning theorem and Holevo's bound, see chapter 12.1 in [25]. Holevo's theorem [181] sets an upper bound on the accessible information $I(A: B)$ transmitted from $A$ to $B$ via a quantum mixed state $\rho$. More precisely, let $\rho=\sum_{t \in \mathcal{T}} p_{t} \rho_{t}$ be a convex combination of mixed states $\rho_{t}$ with positive weights $0 \leq p_{t} \leq 1$ and $\sum_{t} p_{t}=1$. One can think of $\rho$ as the quantum encoding of a random letter drawn from an alphabet $\mathcal{T}$ according to the probabilities $p_{t}$. Let $A$ denote the associated random variable taking values $t$ in $\mathcal{T}$ distributed w.r.t. $p_{t}$. The task is to recover the letter by an optimal POVM measurement performed on $\rho$. Let the (random) measurement outcome be denoted by the (classical) random variable $B$, then Holevo's bound states

$$
I(A: B) \leq S(\rho)-\sum_{t} p_{t} S\left(\rho_{t}\right)=: \chi
$$

The left hand side denotes the classical mutual information $I(A: B)=H(A)+H(B)-H(A, B)$ between the random variables $A$ and $B$ in terms of the Shannon entropy $H$. The Holevo quantity $\chi$ is positive due to the concavity of the von Neumann entropy $S$. If $I(A: B)$ is equal to the Shannon entropy $H(A)$ of the random letter, then the measurement $B$ reveals all information 
about the random letter. In general, however, $S(\rho)-\sum_{t} p_{t} S\left(\rho_{t}\right) \leq H\left(p_{t}\right)$, and equality holds if, and only if, the supports of the $\rho_{t}$ 's are mutually orthogonal, cf. Theorem 11.10 in [25]. If the supports are not mutually orthogonal, then Holevo's bound implies that $I(A: B)$ is strictly less then $H(A)$, meaning that the message cannot be recovered with arbitrary fidelity. This can be traced back to the fact that different $\rho_{t}$ 's are not perfectly distinguishable by POVMs whenever their supports are not mutually orthogonal. In the extreme case, when $\rho_{t}$ are equal for all $t \in \mathcal{T}$, then $\chi=0$, such that even the optimal POVMs measurement protocol on $\rho$ cannot reveal anything about $A$.

Quantum clocks. We apply Holevo's bound to interpret the Shannon entropy $S(\rho)$ of the history matrix $\rho=\sum_{t} p_{t}\left|\psi_{t}\right\rangle\left\langle\psi_{t}\right|$, cf. Figs. 5.1 and 5.2. Imagine Alice draws a random time $t$ from the alphabet $\mathcal{T}$ with probability $p_{t}$. This random time is represented by the mixed state $\rho^{A}=$ $\sum_{t} p_{t}|t\rangle\langle t|$, as a convex combination of mutually orthogonal, i.e. perfectly distinguishable, pure states $|t\rangle\langle t|$ in $\mathcal{H}_{T}$. Alice encodes the random time as $\rho^{B}=\sum_{t} p_{t}\left|\psi_{t}\right\rangle\left\langle\psi_{t}\right|$ in terms of evolving pure states $\left|\psi_{t}\right\rangle\left\langle\psi_{t}\right|$ of a quantum system on $\mathcal{H}_{S}$, and sends it to Bob. Contrary to $\rho^{A}$, where $|t\rangle\langle t|$ have mutually orthogonal support, $\left|\psi_{t}\right\rangle$ are not mutually orthogonal. In other words, one may view $|t\rangle\langle t|$ as a perfect external clock, while the system states $\left|\psi_{t}\right\rangle\left\langle\psi_{t}\right|$ function as an internal (imperfect) system clock 4 . In view of (5.17) with $\rho_{t}=\left|\psi_{t}\right\rangle\left\langle\psi_{t}\right|$ pure, hence, $S\left(\rho_{t}\right)=0$, one concludes that $\chi=S(\rho)$ is the upper bound that a measurement of $\rho$ can reveal about the randomly sampled time $t$. In this sense, $S(\rho)$ is a measure for the precision of the internal quantum clock being send to Bob.

Quantum speed limits. Intuitively, the internal clock works best, if the system state $\left|\psi_{t}\right\rangle$ traverses as many as possible (almost) mutually orthogonal states as a function of the external time parameter $t$. However, the unitary Schrödinger time evolution sets a fundamental quantum speed limit on the dynamics. The minimal time $\tau$ necessary for an initial state $\left|\psi_{0}\right\rangle$ to develop into an orthogonal state $\left|\psi_{\tau}\right\rangle=e^{-i \tau H}\left|\psi_{0}\right\rangle$ under unitary evolution, is limited by the energy variance $\operatorname{var} E=\left\langle(H-\langle H\rangle)^{2}\right\rangle$, and the mean excess energy $\langle E\rangle=\left\langle H-E_{0}\right\rangle$ above the ground state $E_{0}$. The two bounds $\tau \geq \frac{\pi \hbar}{2 \operatorname{var} E}$ [187, and $\tau \geq \frac{\pi \hbar}{2\langle E\rangle}$ [183 are referred to as the Mandelstam-Tamm, and the Margolus-Levitin bound, respectively. These are fundamental quantum mechanical limits, similar to Heisenberg's fundamental uncertainty relation between complementary observables.

These quantum speed limits also have practical implications on the speed of a quantum computer. Because every quantum algorithm that solves a non-trivial problem, involves a state that traverses a minimal number of (almost) mutually orthogonal states in the course of information processing.

Remark about local vs. non-local measurements. Note that the POVM measurement used in Holevo's bound is not constrained to be local or to have a simple physical meaning. Non-local measurements, which may not correspond to any physically meaningful quantity, are explicitly allowed. Furthermore, Holevo's bound does not specify what the optimal POVM measurement is, nor whether Holevo's quantity $\chi$ can be achieved by a measurement 188. In other words, the entropy $S(c)=\chi$ of the reduced history matrix constitutes a global criterion on the nature of the quantum dynamics. As such $S(c)$ depends only on the Hamiltonian's spectrum, and not, e.g., on the spatial morphology of its eigenstates. More refined quantities that classify the quantum dynamics based on, e.g., local properties cannot depend solely on the spectrum.

Notwithstanding, for physically motivated Hamiltonians, the properties of the spectral density are not independent of the energy eigenfunctions. One exemplification of this fact is the statistical distribution of gaps between consecutive energy eigenstates, which is not independent of the

\footnotetext{
${ }^{4}$ This reminds of Page's and Wootter's original motivation to study the emergence of time-dependent states in enlarged Hilbert spaces.
} 
structure of the corresponding eigenfunctions. More precisely, if the gap distribution shows a level repulsion phenomenon, the associated eigenfunctions are typically extended in real space, such that their support in real space overlap significant. Metaphorically speaking, repulsion between energies requires 'communication' of their respective eigenfunctions, as is provided by the overlap of their support. On the contrary, in the absence of level repulsion, the associated eigenfunctions are localized and their real space supports are (nearly) disjoint.

In the following sections we will make explicit use of the level gap statistics, and see how it impacts the dynamics of $S(c)$ when $c$ becomes comparable to the Heisenberg time. 


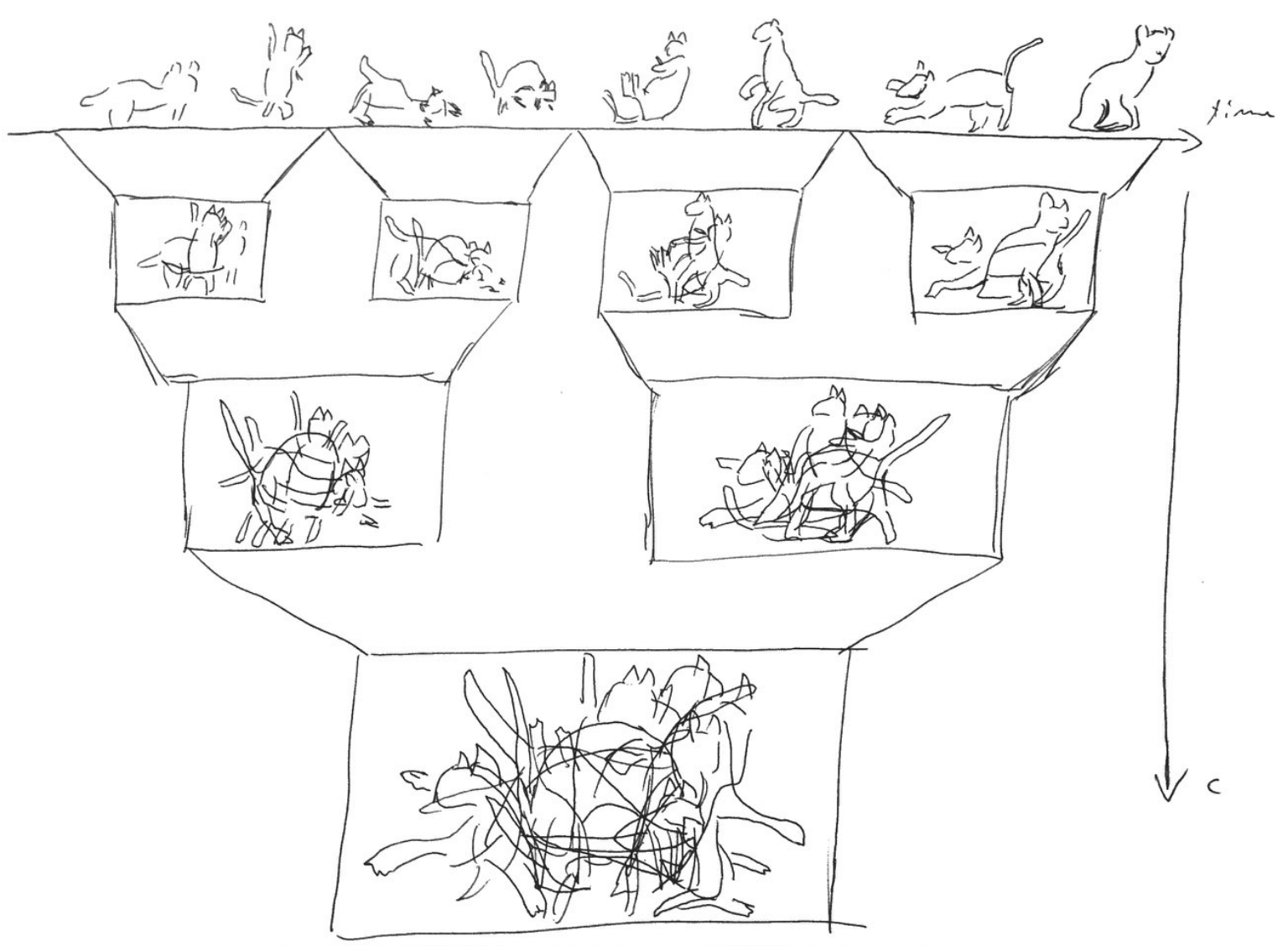

Figure 5.1: Pictorial visualization of the history matrix, and the information theoretic interpretation of its entropy. The unitary quantum evolution $\left|\psi_{t}\right\rangle=\mid$ cat $\left._{t}\right\rangle$ is depicted by snapshots of the state at equidistantly sampled times (upper row). This family of states can be represented by a single time independent Feynman Kitaev history state $|\Psi\rangle \sim \sum_{t}\left|\psi_{t}\right\rangle|t\rangle$ in the extended Hilbert space, where the $t$ summation runs over a time window of effective size $c$, and the mutually orthonormal time states $|t\rangle$ serve as a bookkeeping device to discriminate states at different instants. When tracing out the time labels in the pure state $|\Psi\rangle\langle\Psi|$, one obtains the history matrix $\rho \sim \sum_{t}\left|\psi_{t}\right\rangle\left\langle\psi_{t}\right|$ (second row), in which the states $\left|\psi_{t}\right\rangle\left\langle\psi_{t}\right|$ at different times are incoherently superposed and all information about the temporal order is lost. Different boxes within a row depict history matrices associated to translated time windows, and, hence, due to translation invariance, cf. table 5.1, have identical entropies. According to Holevo's bound, cf. Eq. (5.17) and the subsequent discussion, the entropy $\chi$ of $\rho$ bounds the accessible information on the instants of time that can be obtained by a POVM measurement on $\rho$. As the effective size $c$ of the time window is enlarged (third and fourth row), the entropy of $\rho$ increases. Depending on the dynamics of the underlying quantum evolution, $\chi$ behaves differently as a function of $c$. The active and unpredictable nature of the cat is a metaphor for a quantum state with (i) high excess energy above the ground state, and (ii) overlaps in the bulk spectrum of a non-integrable quantum Hamiltonian obeying level repulsion. For large values of $c$ comparable to the Heisenberg time, $\chi$ approaches its maximal value as a power law. In systems with level repulsion the power law is inversely quadratic in $c$, cf. Figs. 5.6 and 5.9. This is in contrast to systems with Poisson distributed gaps, where the power law is inversely linear, cf. Fig. 5.2 . 


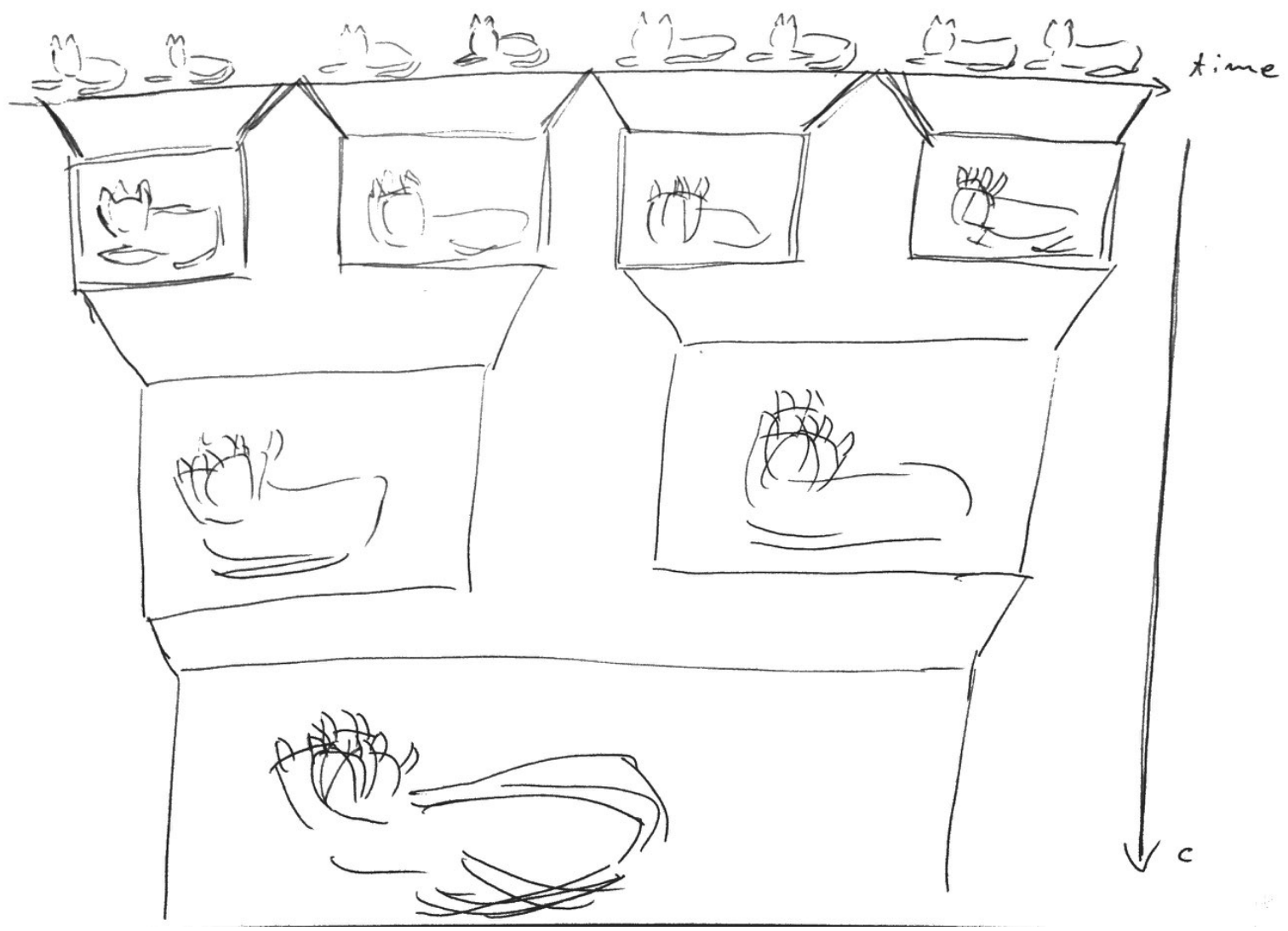

Figure 5.2: Similar schematic sketch as Fig. 5.1. In contrast to Fig. 5.1 the cat is less active, and symbolizes states with (i) small excess energy above the ground state, and (ii) in the (integrable) region of the Hamiltonian's spectrum with Poisson distributed level gap statistics. In systems with Poisson distributed gaps the entropy of the time reduced history state approaches its maximum inversely proportional as a function of the time window size $c$. 


\subsection{Wigner random matrix}

In this section we investigate the history entanglement of an initial state $\left|\psi_{0}\right\rangle$ with uniform energy distribution in the bulk spectrum of a random Wigner matrix. We are not interested in the Wigner matrix as a specific realized physical Hamiltonian. The idea is rather to use the Wigner matrix as a model for universal local spectral properties observed in numerous generic non-integrable quantum systems 100, 101, 103, 189, 190. In the same way Wigner did not study random matrices as a realistic Hamiltonian of a heavy nucleus [45, but rather as a mathematically amenable model to compute its universal spectral properties that are independent of microscopic details and on only depend on the symmetry.

Universal spectral properties of Wigner matrices. We consider the spectrum of the $N$ by $N$ Hermitian, random Wigner matrix $H$, i.e. the matrix elements $H_{i j}$ are independent (modulo the constraint $H_{i j}=\bar{H}_{j i}$ ) and identically distributed with

$$
\mathbb{E} H_{i j}=0, \quad \text { and } \quad \mathbb{E}\left|H_{i j}\right|^{2}=\frac{1}{N} .
$$

The scaling of the variance guarantees that the typical eigenvalues are of order one as $N \rightarrow \infty$. In particular, the spectral density converges (weakly) to the semicircle law, $d(E)=\frac{1}{N} \sum_{i} \delta\left(E-E_{i}\right) \rightarrow$ $\rho_{\mathrm{sc}}(E)=\frac{1}{2 \pi} \sqrt{\left(4-E^{2}\right)_{+}}$, which has compact support, see Fig. 5.3. This result is universal in the sense that it is independent of the precise distribution of $H_{i j}$.

Two famous results about the local statistics of the eigenvalues of Wigner matrices concern the gap statistics (Wigner surmise) 123

$$
\mathbb{P}\left[N \bar{d}\left(E_{j}\right) \cdot\left(E_{j}-E_{j+1}\right)=\Delta+d \Delta\right] \rightarrow P_{\beta=2}(\Delta) d \Delta \approx \frac{32 \Delta^{2}}{\pi^{2}} e^{-4 \Delta^{2} / \pi} d \Delta
$$

and the (label independent) correlations 191

$$
\begin{aligned}
& \mathbb{P}\left(\exists i \neq j: E_{i}=x+d x, E_{j}=y+d y\right)=p_{N}^{(2)}(x, y) d x d y \\
& \frac{1}{\bar{d}(E)^{2}} p_{N}^{(2)}\left(E+\frac{x_{1}}{N \bar{d}(E)}, E+\frac{x_{2}}{N \bar{d}(E)}\right) \rightarrow \operatorname{det}\left(\operatorname{sinc}\left[\pi\left(x_{i}-x_{j}\right)\right]_{i, j \in 1,2}\right),
\end{aligned}
$$

where $\bar{d}(E)=\mathbb{E} \sum_{i} \delta\left(E-E_{i}\right) / N$. Note, that the energies in (5.19) and (5.20) are rescaled by the local mean distance $[N \bar{d}(E)]^{-1}$ between consecutive energies close to $E$. With this rescaling the result becomes universa $5^{5}$ in the sense that the right hand side of $(5.20)$ does not depend on energy $E$, but only on the difference $\left(x_{1}-x_{2}\right)$. Also, since $1-\operatorname{sinc}\left[\pi\left(x_{1}-x_{2}\right)\right]^{2}$ does not factorize, eigenvalues are locally correlated. More precisely, eigenvalues repel at small distances as a consequence of the quadratic scaling $\propto\left(x_{1}-x_{2}\right)^{2}$.

Eq. (5.19) should be compared with the gap distribution

$$
\mathbb{P}\left[N \bar{d}\left(E_{j}\right) \cdot\left(E_{j}-E_{j+1}\right) \rightarrow \Delta+d \Delta\right] \rightarrow e^{-\Delta} d \Delta,
$$

of the Poisson point process on an interval $\Delta E$ with flat density of states $\bar{d}(e)=1 /|\Delta E|$. The Poisson point process is uncorrelated (on all scales),

$$
\frac{1}{\bar{d}(E)^{2}} p_{N}^{(2)}\left(E+\frac{x_{1}}{N \bar{d}(E)}, E+\frac{x_{2}}{N \bar{d}(E)}\right) \rightarrow 1 .
$$

\footnotetext{
${ }^{5}$ The local spectral property 5.20 also holds for other Hermitian matrix ensembles such as generalized Wigner matrices and (non Gaussian) invariant ensembles 192,193.
} 


\section{History state entanglement}

Remark on label dependence and independence. Note that the gap statistics is a label dependent correlation of energy levels, while the correlation function $(5.20)$ and more generally $(5.29)$ are label independent. The gap distribution function can be expressed in terms of the $k$-point correlators of all orders $[122,123]$.

Remark on GOE statistics. For real symmetric Wigner matrices the global eigenvalue density still converges to the semicircle law, while the local statistics follows a slightly different law. In particular, the Wigner surmise is $P_{\beta=1}(\Delta) \approx \pi \Delta / 2 e^{-\pi \Delta^{2} / 4}$ in the real symmetric case 123, 192 . The correlations (5.20) still take the form of a determinant, but the sinc kernel is replaced by a more complicated function 191,193. As the subsequent reasoning does not depend on the precise form of the kernel, we focus on the simpler complex case.

The local eigenvalue statistics influences the entropy $S_{k}(c)$ as $c$ becomes comparable to the inverse mean distance between eigenvalues, i.e. for $c \gtrsim N \bar{d}$, cf. (5.11). In particular, for $c \rightarrow \infty$, the entanglement entropy saturates to its maximum $\lim _{c \rightarrow \infty} S_{k}(c)$, faster in the presence of level repulsion than in the absence of level repulsion. Indeed, we now show that the saturation follows a power law with different exponents for the Wigner and Poisson case.

We focus on the second Rényi (collision) entropy, cf. (5.13), with uniform time window function $\Phi_{\text {box }}$,

$$
\begin{aligned}
\exp \left[-S_{2}(c)\right] & =\frac{1}{N^{2}} \sum_{i, j} \operatorname{sinc}\left[\left(E_{i}-E_{j}\right) c / 2\right]^{2} \\
& =\frac{1}{N}+\frac{N-1}{N} \frac{1}{N(N-1)} \sum_{i \neq j} \operatorname{sinc}\left[\left(E_{i}-E_{j}\right) c / 2\right]^{2}
\end{aligned}
$$

It is plausible that the sum $\frac{1}{N(N-1)} \sum_{i \neq j} \operatorname{sinc}\left[\left(E_{i}-E_{j}\right) c / 2\right]^{2}$ is self-averaging in the sense that it obeys a large deviation principle $e^{6}$. We may thus replace the sum by its expectation value and use $\frac{1}{N(N-1)} \mathbb{E} \sum_{i \neq j} f\left(E_{i}, E_{j}\right)=\int f\left(x_{1}, x_{2}\right) p_{N}^{(2)}\left(x_{1}, x_{2}\right) d^{2} x$ (for any symmetric function $f$ ) to obtain

$$
\begin{aligned}
& \approx \frac{1}{N}+\frac{N-1}{N} \int \operatorname{sinc}\left[\left(x_{1}-x_{2}\right) c / 2\right]^{2} p_{N}^{(2)}\left(x_{1}, x_{2}\right) d^{2} x \\
& =\frac{1}{N}+\frac{N-1}{N} \int d E \int \frac{d x}{N \bar{d}(E)} \operatorname{sinc}\left[\frac{x}{N \bar{d}(E)} \frac{c}{2}\right]^{2} p_{N}^{(2)}\left(E, E+\frac{x}{N \bar{d}(E)}\right),
\end{aligned}
$$

after a change of variables $\left(x_{1}, x_{2}\right)=(E, E+x /(N \bar{d}(E))) \mapsto(E, x)$. The last integral can be solved in the limit $c, N \rightarrow \infty$ with the help of Eqs. 5.20) and (5.22) while keeping $c / N$ of order one.

Wigner. Using (5.20) in (5.24), yields

$$
S_{2}(c)=\log N-\log \left[\int d E \int d x \bar{d}(E)\left(\operatorname{sinc} \frac{x}{2 \bar{d}(E)} \frac{c}{N}\right)^{2}\left[1-(\operatorname{sinc} \pi x)^{2}\right]\right]
$$

\footnotetext{
${ }^{6}$ The energies $E_{i}$ are independent on a large scale of separation, they are only dependent on a local scale as stated above. Moreover, the Lipschitz norm of $f(x)=\sum_{i \neq j} \operatorname{sinc}\left(\left(x_{i}-x_{j}\right) c / 2\right)^{2} / N /(N-1)$ is bounded by $\|f\|_{\text {Lip }}<$ const $/ N$. Hence, e.g. by Talagrand's concentration inequality, $f\left(E_{1}, \cdots E_{N}\right)$ obeys a large deviation principle.
} 
The $x$ integration can be performed explicitly by noting

$$
\int(\operatorname{sinc} \alpha x)^{2}(\operatorname{sinc} x)^{2}=\pi \begin{cases}1-\alpha / 3, & 0 \leq \alpha<1 \\ 1 / \alpha-1 /\left(3 \alpha^{2}\right), & 1 \leq \alpha\end{cases}
$$

The distinction between the two cases $c / N<2 \pi \bar{d}$ and $c / N>2 \pi \bar{d}$ occurs naturally and is consistent with conjugate relation between time and energy. We divide the support of $\bar{d}$ into the set $\mathcal{D}=$ $\{E: c / N>2 \pi \bar{d}(E)\}$ of all energies for which the local density is small compared to $c / N$, and its disjoint complement $\mathcal{D}^{c}=(\operatorname{supp} \bar{d}) \backslash \mathcal{D}$. Then,

$$
\begin{gathered}
S_{2}(c)=\log N-\log \left[1+(c / N)^{-2} A_{-2}+(c / N)^{-1} A_{-1}+A_{0}+(c / N) A_{1}\right], \\
A_{-2}=\frac{4 \pi^{2}}{3} \int_{\mathcal{D}} \bar{d}^{3}, \quad A_{-1}=2 \pi \int_{\mathcal{D}^{c}} \bar{d}^{2}, \quad A_{0}=\int_{\mathcal{D}^{c}} \bar{d}^{1}, \quad A_{1}=\frac{1}{6 \pi} \int_{\mathcal{D}^{c}} \bar{d}^{0}
\end{gathered}
$$

The limiting cases $c / N>2 \pi \max \bar{d}$ and $c / N<2 \pi \min \bar{d}$ are

$$
S_{2}(c)=\log N-\log \left[1+(c / N)^{-2} A_{-2}\right], \quad A_{-2}=\frac{4 \pi^{2}}{3} \int \bar{d}^{3}
$$

and

$$
S_{2}(c)=\log N-\log \left[(c / N)^{-1} A_{-1}+(c / N) A_{1}\right], \quad A_{-1}=2 \pi \int \bar{d}^{2}, A_{1}=\frac{\operatorname{supp} \bar{d}}{6 \pi},
$$

respectively. In particular, 5.26a yields the power law $\log N-S(c) \propto(c / N)^{-2}$ for large $c$, and $5.26 \mathrm{~b}$ entails $\operatorname{logarithmic}$ growth $S_{2}(c) \approx \log (c)-\log A_{-1}$ for small $c$.

Poisson. Using $(5.22)$ in 5.24$)$, yields

$$
S_{2}(c)=\log N-\log \left[1+(c / N)^{-1} A_{-1}\right], \quad A_{-1}=2 \pi \int \bar{d}^{2} .
$$

In particular, $\log N-S_{2}(c) \propto(c / N)^{-1}$ for large $c$ is a different power law than in the case of the Wigner matrix, see Fig. 5.6 .

Figs. 5.5 and 5.6 shows numerical data and confirms the validity of Eqs. (5.25) and (5.27).

Remark (about large $c$ ). The numerical results in Figs. 5.5 and 5.6 are obtained for finite $N$. When $c$ is too large, finite size effects become visible and the numerical data starts to deviate from the analytical predictions, see Fig. 5.7. The finite size effects depend on the individual samples of the Wigner matrix $H$ and the self-averaging breaks down. Essentially, the finite size effect is due to the fact that the statistics of energies is insufficient on a too small scale. In particular, for finite $N$ there exists a minimal distance $\Delta E_{\min }$ (its exact value depends on the realization of randomness) between consecutive eigenvalues. Hence, the correlations $p_{N}^{(2)}\left(E+x_{1} / N \bar{d}, E+x_{2} / N \bar{d}\right) / \bar{d}^{2}$ vanish exactly for $\left(x_{1}-x_{2}\right) / N \bar{d}<\Delta E_{\min }$, while the asymptotic limit predicts $\left(1-\operatorname{sinc}\left[\pi\left(x_{1}-x_{2}\right)\right]^{2}\right) \neq 0$. This discrepancy between the numerical and the analytical value is the main contribution to the integral in (5.24) for $c \gtrsim 2 \pi / \Delta E_{\min }$. Thus, for fixed $N$ and a fixed realization of a $N$ by $N$ Wigner matrix $H$ with minimal distance $\Delta E_{\min }$ between two consecutive eigenvalues, the result (5.25) can only agree with the numerical data for $c \lesssim 2 \pi / \Delta E_{\min }$. Analogously, $c \lesssim 2 \pi / \Delta E_{\min }$ is necessary for the validity of (5.27). 


\section{History state entanglement}

Remark (about small $c$ ). To arrive at the results (5.25) and (5.27), we have used the information about correlations between energies on a local energy scale $\sim(N d)^{-1}$. This information is only accessible when $c$ is of the order of the typical inverse level spacing, i.e. for $c / N \gtrsim \bar{d}$. For small $c$ the results (5.25) and (5.27) fail to approximate $S_{2}(c)$. In order to get the behavior of $S_{2}(c)$ for $c / N \lesssim \bar{d}$, one does not need to know the correlations $p_{N}^{(2)}$ on a local scale, instead the density of states $d(E)$ on a global scale suffices. More specifically,

$$
\begin{aligned}
e^{-S_{2}(1, c)} & =\int d^{2} E \operatorname{sinc}\left[\left(E_{1}-E_{2}\right) c / 2\right]^{2} d\left(E_{1}\right) d\left(E_{2}\right) \\
& =1-\operatorname{var}(E) \frac{c^{2}}{6}+\mathcal{O}\left(c^{4}\right),
\end{aligned}
$$

Thus, for small $c / N \ll \bar{d}$ the entropy $S_{2}(c)$ depends on the energies only through the variance and is independent of the details of $d(E)$.

Remark. Higher Rényi entropies can be obtained analogously by using the general form

$$
\frac{1}{\bar{d}(E)^{k}} p_{N}^{(k)}\left(E+\frac{x_{1}}{N \bar{d}(E)}, \cdots, E+\frac{x_{k}}{N \bar{d}(E)}\right) \rightarrow \operatorname{det}\left(\operatorname{sinc}\left[\pi\left(x_{i}-x_{j}\right)\right]_{i, j=1, \cdots k}\right)
$$

of $k$-point correlations in the Wigner case and the $k$-point independence in the Poisson case, respectively.

Gap statistics. The different scaling of $S_{2}(c)$ with $c / N$ in Eqs. 5.25) and (5.27) can also be obtained from the gap distributions (5.19) and (5.21), respectively. To get the correct amplitudes and the crossover from 5.26a to 5.26b), it is necessary to know the precise correlations and not just the gap distribution.

We elaborate more on the fact that the power law is a consequence of the gap statistics. In the large $c$ limit the main contribution to the summation in 5.23) comes from small energy differences,

$$
\begin{aligned}
\frac{1}{N^{2}} \sum_{i \neq j} \operatorname{sinc}\left[\left(E_{i}-E_{j}\right) c / 2\right]^{2} & \approx \frac{1}{N^{2}} \sum_{i} \operatorname{sinc}\left[\left(E_{i}-E_{i+1}\right) c / 2\right]^{2}+\operatorname{sinc}\left[\left(E_{i}-E_{i-1}\right) c / 2\right]^{2} \\
& =\frac{2}{N^{2}} \sum_{i} \int \operatorname{sinc}\left(\frac{\Delta}{N d\left(E_{i}\right)} \frac{c}{2}\right)^{2} P(\Delta) d \Delta
\end{aligned}
$$

The unfolded energy gaps $\Delta_{i}=\left(E_{i+1}-E_{i}\right) N d\left(E_{i}\right)$ are distributed according to $P(\Delta) d \Delta$. For the sake of the argument, we assume that the spectral density $d(E)=|\operatorname{supp} d|^{-1}$ is constant (i.e. independent of energy) on its support $\operatorname{supp} d=\left[E_{\min }, E_{\max }\right]$, such that the last expression simplifies to

$$
\frac{2}{N} \int \operatorname{sinc}\left(\Delta \pi c / t_{H}\right)^{2} P(\Delta) d \Delta,
$$

where $t_{H}=2 \pi /\langle\Delta E\rangle$ is the Heisenberg time, inversely proportional to the mean energy spacing $\langle\Delta E\rangle=\left(E_{\max }-E_{\min }\right) / N$. This integral is evaluated in the large $c / t_{H}$ limit for $P(\Delta)=$ $c_{1} \Delta^{\beta} e^{-c_{2} \Delta^{\gamma}}$, where $c_{2}$ is a positive constant and $c_{1}$ is fixed by normalization. Different values of $(\beta, \gamma)=(1,2),(2,2)$, and $(0,1)$ correspond to GOE, GUE, and Poisson gap statistics, respectively. We find the leading scaling

$$
\int \operatorname{sinc}\left(\Delta \pi c / t_{H}\right)^{2} \Delta^{\beta} \exp \left(-c_{2} \Delta^{\gamma}\right) d \Delta \propto \begin{cases}c^{-2}, & 1 \leq \beta \\ c^{-\beta-1}, & 0<\beta \leq 1\end{cases}
$$


as $c / t_{H} \rightarrow \infty$, i.e. when $c$ is large in units of the Heisenberg time $t_{H}$. In particular, this confirms the results (5.26a) and (5.27) obtained for the GUE and Poisson statistics. It also extends the result for the GUE ensemble to the real symmetric GOE ensemble.

The intuition behind (5.30) is instructive. We argue that the scaling for $\beta<1$ is caused by the small gap statistics, while the scaling for $1<\beta$ is a consequence of the statistics of large gaps. The $\operatorname{GOE}(\beta=1)$ statistics demarcates the two scenarios.

On the one hand, for $0<\beta<1$ the integral $\int_{\mathbb{R}_{+}} x^{\beta} \operatorname{sinc}(x)^{2}$ converges to a finite value, say $C_{\beta}$. Hence, the second half of $(5.30)$ is obtained by a change of integration $\Delta \mapsto x=\Delta \pi c / t_{H}$ and expanding the exponential $\exp \left[-c_{2} x^{\gamma} /\left(\pi c / t_{H}^{\gamma} / \widetilde{c}\right)^{\gamma}\right]$ for large $c / t_{H}$. This shows, in particular, that the scaling behavior $\propto C_{\beta} c^{-1-\beta}$ is caused by the statistics of short gaps.

On the other hand, for $1<\beta$ the integral $\int_{\mathbb{R}_{+}} x^{\beta} \operatorname{sinc}(x)^{2}$ does not converge due to the slow inversely proportional decay of the sinc function's envelope. This divergence is regularized by the additional exponential factor in the integrand of (5.30), and the dominant contribution to the integral must come from the tail of the integrand in the large $c / t_{H}$ limit. Therefore, the scaling of the first half of 5.30 follows from $\int\left(x \pi c / t_{H}\right)^{-2} x^{\beta} \exp \left(-c_{2} x^{\gamma}\right) d x \propto c^{-2}$. In other words, the large gap statistics determines the scaling of 5.30 for $\beta>1$.

Eq. 5.30 has also been confirmed numerically.

GUE $(\beta=2)$

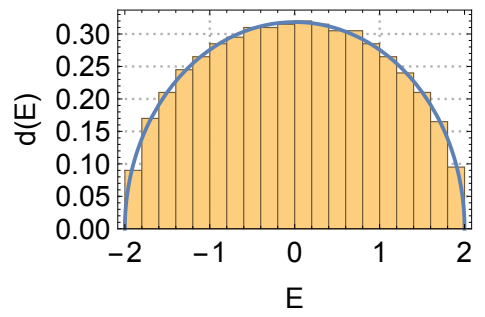

GUE $(\beta=2)$

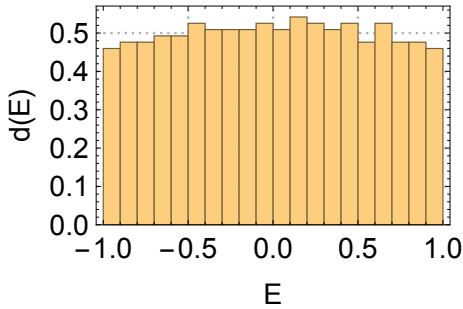

Poisson

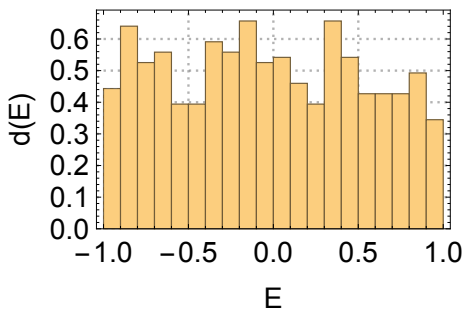

Figure 5.3: Normalized density of states $d(E)$ of a $10^{3}$ by $10^{3}$ Wigner matrix, cf. Eq. (5.18) (left), obeying the semicircle law (solid blue line). Density of states of the bulk spectrum containing $N=608$ eigenvalues between -1 and 1 (middle). Density of states of a spectrum coming from a Poisson point process with $N=608$ on the interval $[-1,1]$ (right). The mean density of the bulk Wigner spectrum and the Poisson spectrum are similar, but differ in terms of their local statistics, cf. Fig. 5.4 . 

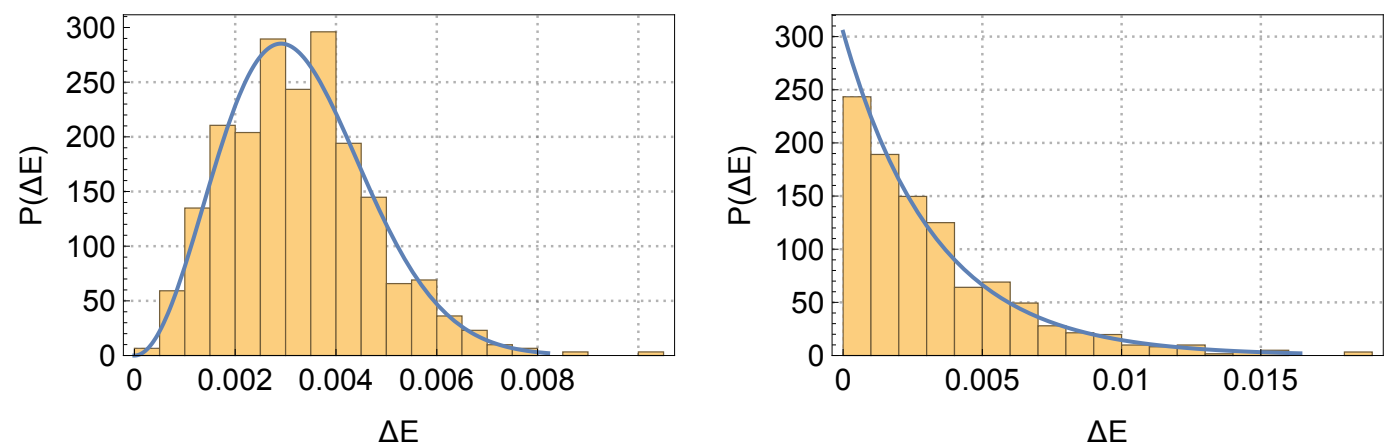

Figure 5.4: Histogram of gaps between consecutive levels the bulk spectrum $(N=608)$ of a Wigner matrix (left) and an uncorrelated Poisson spectrum (right) with similar average density as shown in Fig. 5.3. Wigner surmise, cf. Eq. (5.19) (solid blue line, left), and Poisson distribution, cf. Eq. (5.21) (solid blue line, right) are guides to the eye.
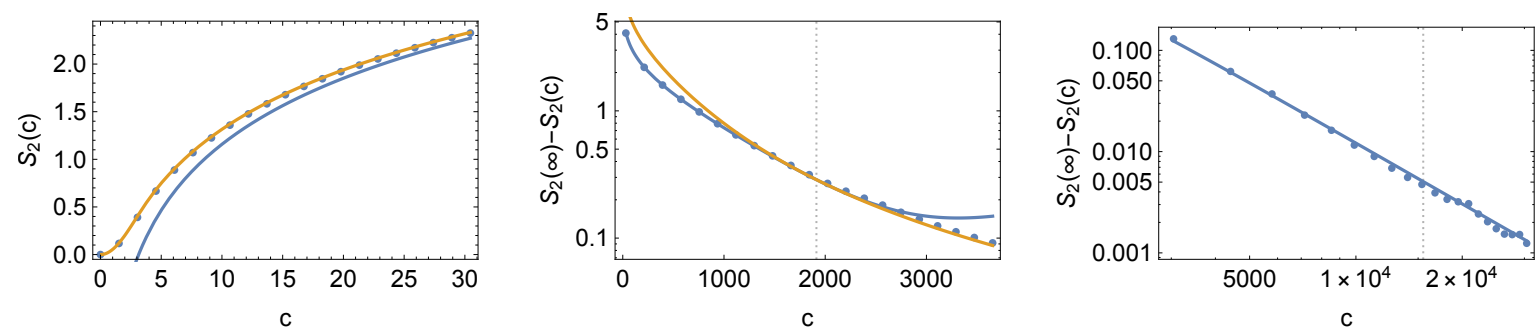

Figure 5.5: Second Rényi entropy $S_{2}(c)$ for bulk of Wigner spectrum obtained numerically for $N=608$ (blue dots). Regime of small $c$ (left) with analytical predictions Eq. (5.28) (solid orange line), and Eq. (5.26b) (solid blue line). Regime of intermediate $c$ (middle) shows the crossover from Eq. (5.26b) (solid blue line) to Eq. (5.26a) (solid orange line). The vertical dotted line is at $c=2 \pi /\langle\Delta E\rangle=2 \pi N \bar{d}$ (Heisenberg time). Regime of large $c$, i.e. $c / N>2 \pi \max d$, (right) shows power law behavior according to Eq. (5.26a). Vertical dotted line is at $2 \pi / \min (\Delta E)$, inversely proportional to the minimal gap. 


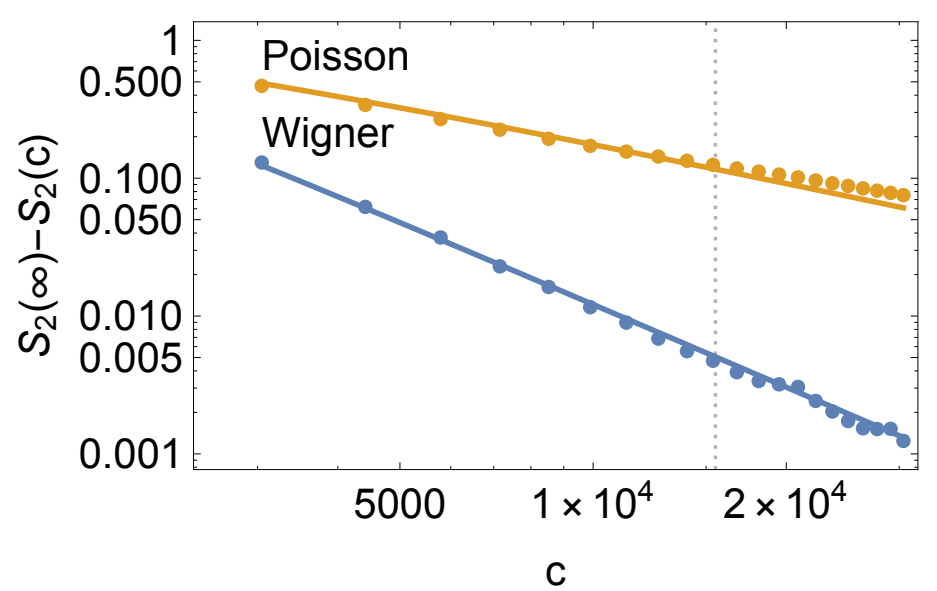

Figure 5.6: Different power law saturation of the Rényi entropy for the Wigner (blue) and Poisson (orange) case for large $c / N$. Numerical results for $N=608$ (dots) agree with the analytical prediction (5.26a) (solid blue line) and (5.27) (solid orange line). Vertical dotted line at $c=2 \pi / \min (\Delta E)$ indicates when finite size effects become important for the Wigner case. Finite size effects lead to the discrepancy between the numerical data and the analytic prediction. Due to the lack of level repulsion, the minimal gap $\min (\Delta E)$ is much smaller for the Poisson case $(2 \pi / \min (\Delta E)$ is outside the range of plotted $c$ ), and finite size effects are less significant for the range of $c$ shown.

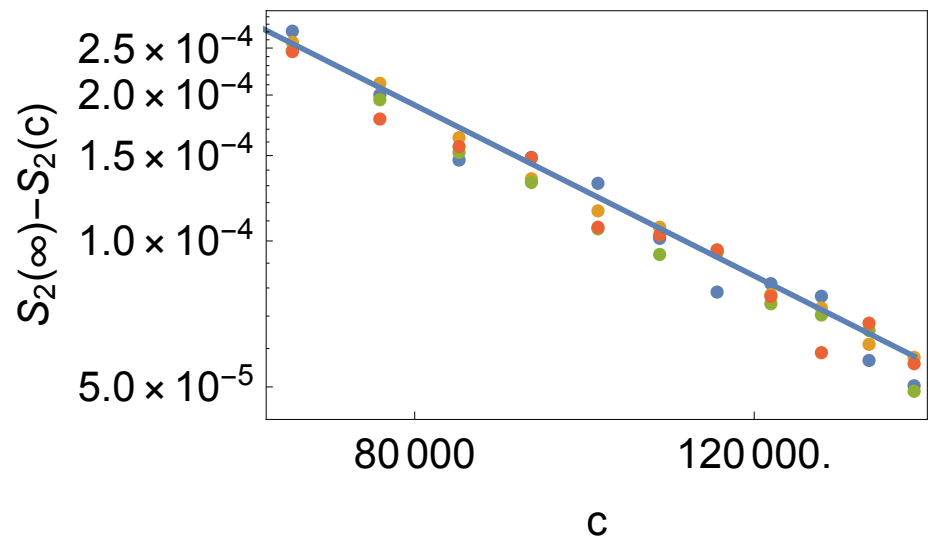

Figure 5.7: Numerical Rényi entropy for the bulk spectrum of Wigner matrix for $N \approx 608$ (dots), and analytical prediction 5.26a (solid blue line). Different colored dots correspond to different realizations of randomness. Finite size effects become more pronounced for large $c / N$. Finite size effects depend on the particular realization of randomness, leading to a break down of self-averaging. 


\subsection{Hardcore boson lattice model}

In the previous section we have seen how the presence and absence of spectral level repulsion implies different power law behavior of the history entanglement $S$ as a function of the time window size, cf. Fig. 5.6. This has been demonstrated explicitly for the spectrum of a Hermitian random Wigner matrix. Random matrices are useful to describe universal local spectral properties of physical systems, such as heavy nuclei [45, 121, chaotic billiards 100,101, and non-integrable quantum many body Hamiltonians [190. However, random matrices cannot capture non-universal properties of physical systems. In fact, physical Hamiltonians differ from random matrix Hamiltonians in some respects. For example, in contrast to local quantum many body Hamiltonians, which are sparse matrices in position basis, random Wigner matrices are dense. In this section we complement the previous discussion by investigating the local quantum many body Hamiltonian of a hardcore boson lattice model. Thereby, we corroborate the intuition that the results of the previous section depend solely on the universal local aspects of the spectrum.

We consider hardcore bosons (i.e. at most one boson is allowed on each site) on a one-dimensional chain of $L$ sites with periodic boundary conditions (i.e. all lattice indices are understood modulo $L)$, evolving according to the Hamiltonian

$$
H=-t \sum_{i}\left(c_{i}^{\dagger} c_{i+1}+\text { h.c. }\right)+V_{1} \sum_{i}\left(c_{i}^{\dagger} c_{i}-\frac{1}{2}\right)\left(c_{i+1}^{\dagger} c_{i+1}-\frac{1}{2}\right)+V_{2} \sum_{i}\left(c_{i}^{\dagger} c_{i}-\frac{1}{2}\right)\left(c_{i+2}^{\dagger} c_{i+2}-\frac{1}{2}\right),
$$

where $c_{i}, c_{i}^{\dagger}$ are bosonic annihilation and creation operators obeying canonical commutation relations and the hardcore constraint $c_{i}^{\dagger 2}=0, t$ is the hopping parameter, and $V_{1}$ and $V_{2}$ are nearest neighbor and next nearest neighbor interaction strengths, respectively. The Hamiltonian is integrable for $V_{2}=0$, and non-integrable for $V_{2} \neq 0$ [190. In either case, the symmetries of the Hamiltonian lead to the conserved quantities of particle number $\widehat{N}=\sum_{i} c_{i}^{\dagger} c_{i}$ (global $U(1)$ symmetry), (crystal) momentum $\widehat{k}$ (translation symmetry), and parity $\widehat{P}$ (reflection symmetry), cf. 194. In addition, at half-filling, i.e. in the subspace spanned by $\mathrm{L} / 2$ eigenstates of $\widehat{N}$, one has particle hole symmetry and the associated conserved quantity $\widehat{Z}=\prod_{j}\left(c_{j}^{\dagger}+c_{j}\right)$ with eigenvalues $\{-1,+1\}$. We confine ourselves to the situation of half-filling, and consider the common 7 eigenbasis of $H$, $\widehat{P}, \widehat{k}$, and $\widehat{Z}$, in which the Hamiltonian is block-diagonal. The eigenspace associated to each block is referred to as (symmetry) sector, and different sectors are referenced by the corresponding eigenvalues of $(\widehat{k}, \widehat{P}, \widehat{Z})$.

Remark (extensive vs. intensive scaling). Two prominent choices of scaling the Hamiltonian as a function of system size $N$ are extensive and intensive. Eq. (5.31) is an example for an extensive Hamiltonian, while (5.18) is intensive. In an extensive system, the Hamiltonian, and hence its spectrum, scales linearly with the system size.

In random matrix theory, one key quantity of interest is the asymptotic empirical spectral density (ESD) in the large $N$ limit. A well defined (weak) limit of the asymptotic ESD on an $N$-independent scale requires intensive scaling. This results in an average spacing of $\mathcal{O}\left(N^{-1}\right)$ between consecutive eigenvalues. Hence, the limit of the asymptotic ESD can only be in a weak sense, i.e. the ESD is tested against smooth functions on scale $\mathcal{O}\left(N^{0}\right)$, where individual eigenvalues cannot be resolved.

For example, the Wigner semicircle law has compact support on $[-2,2]$ in the large $N$ limit.

\footnotetext{
${ }^{7}$ To be more precise, parity and momentum do not commute. Instead of the momentum eigenstates one can consider semi-momentum eigenstates (a superposition of $\pm p$ momentum eigenstates). The semi-momentum commutes with the Parity operator. The zero momentum subspace is identical to the zero semi-momentum subspace. As we are mainly interested in the $\widehat{k}=0$ subspace, the distinction of momentum and semi-momentum is of minor significance for the discussion. More details can be found in 195 .
} 
To achieve this, the typical size of random Wigner matrix elements are of order $1 / \sqrt{N}$, i.e. the variance of matrix entries is of order $1 / N$, such that its eigenvalues are of order on $\AA^{8}$, cf. Eq. (5.18). This $1 / N$ scaling of the variance is also known as mean field scaling.

We have identified qualitatively different regimes of $S_{k}(c)$ depending on how $c$ compares to the maximal, average, and minimal distance between consecutive eigenenergies. As a consequence, these qualitatively different regimes scale with $N$ for intensive systems and are independent of $N$ for extensive systems. Since we do not pursue a detailed analysis of different system sizes, this difference between intensive and extensive systems plays no crucial role.

We want to connect to the discussion of the Wigner matrices of the previous section. It is conjectured, and confirmed by many examples 100,101, 103, 189, 190, that the (bulk) energy level statistics of a typical non-integrable quantum system follows the law of a random matrix ensemble of the same symmetry as the system under consideration. In particular, since the Hamiltonian (5.31) is real (time reversible) and symmetric, one expects $\operatorname{GOE}(\beta=1)$ level statistics. To see a clear GOE statistics, one must investigate the correlations of the (bulk) spectrum within a single, fixed symmetry sector. The reason why one has to confine to a single sector is that due to the block structure of the Hamiltonian, eigenvalues of different symmetry sectors do not 'interact', in contrast to the eigenvalues of a dense Wigner matrix. As a consequence, eigenvalues of different symmetry sectors are not correlated. In particular, they do not repel. Confining to symmetry sectors is also referred to as considering desymmetrized Hamiltonians [92. The effect on the gap statistics of combining different sectors has been investigated in [102], but we avoid this additional difficulty by restricting to a single sector.

In the following we consider the sector of zero momentum $\widehat{k}=0$, even parity $\widehat{P}=1$, and even particle hole symmetry $\widehat{Z}=1$. Among all sectors, this is the sector of the largest dimension (e.g. for $L=22$ the dimension is $N^{\prime}=8359$ ) 195]. We denote the energy eigenvalues of this sector in non-decreasing order by $E_{0}^{\prime} \leq E_{1}^{\prime} \leq \cdots \leq E_{N^{\prime}-1}^{\prime}$. The set of $N=\left\lfloor p N^{\prime}\right\rfloor, 0<p<1$, of these eigenvalues obtained by discarding the first and last $\left\lfloor(1-p) N^{\prime} / 2\right\rfloor$ energies is called the bulk of the spectrum and the energies in the bulk are denoted by $E_{0} \leq E_{1} \leq \cdots \leq E_{N-1}$ in non-decreasing order. Without loss of generality, we assume $E_{0}=0$, which can always be achieved by adding an appropriate, but otherwise irrelevant, constant to $H$. Figure 5.8 confirms that the gap distribution of the bulk spectrum is well described by an exponential distribution, and the $\beta=1$ Wigner surmise in the integrable, and non-integrable case, respectively.

Initial states in the bulk of the symmetry sector are expanded as $\left|\psi_{0}\right\rangle=\sum_{n=0}^{N-1} c_{n}\left|E_{n}\right\rangle$. In the following, we study two particular initial states. First, uniform states for which $c_{n}=1 / \sqrt{N}$. And second, Margolus-Levitin states with

$$
c_{n}=\sqrt{\frac{E_{n+1}-E_{n}}{E_{N-1}}} \text { for } 0 \leq n \leq N-2, \quad \text { and } \quad c_{N-1}=0 .
$$

Note that the state is indeed normalized, i.e. $\sum_{n=0}^{N-1}\left|c_{n}\right|^{2}=1$, because $E_{0}=0$. The naming of the latter state is motivated by Ref. [183, in which Margolus and Levitin investigate quantum speed limits. They show that the time $\tau$ required for an evolving quantum state to pass through $N$ different mutually orthogonal states, is bounded by $\tau \geq \pi \hbar / E$, where $E$ is the mean energy $E=\langle H\rangle$ of the Hamiltonian with zero ground state energy, $E_{0}=0$. This limit is saturated for the harmonic

${ }^{8} \mathrm{~A}$ simple heuristic argument goes as follows. For a Hermitian random Wigner matrix $H$, the trace of $H H^{\dagger}$ is written in two different equivalent ways. First, as $\operatorname{Tr} H H^{\dagger}=\sum_{i j}\left|H_{i j}\right|^{2}$, and second, in terms of its eigenvalues $E_{j}$ as $\operatorname{Tr} H H^{\dagger}=\sum_{j} E_{j}^{2}$. Taking the expectation of both expressions shows that $\sum_{i j}$ var $H_{i j}$ must be of order $\mathcal{O}(N)$ in order for $E_{j}$ to scale as $\mathcal{O}\left(N^{0}\right)$. Hence, var $H_{i j}=\mathcal{O}\left(N^{-1}\right)$. 

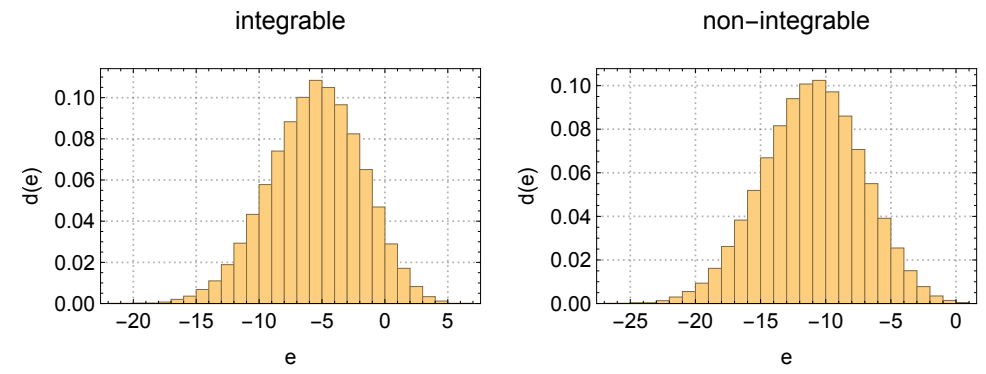

integrable (bulk)
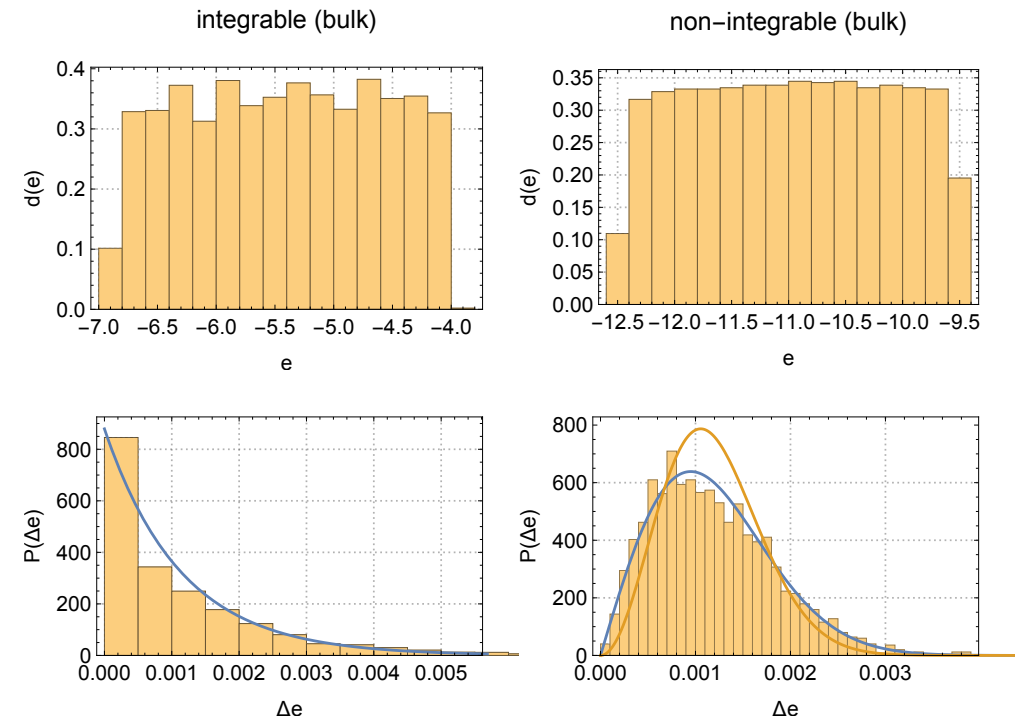

Figure 5.8: Density of states (upper row) for the $(\widehat{k}, \widehat{P}, \widehat{Z})=(0,1,1)$ symmetry sector of the hardcore boson model (5.31) on $L=22$ sites for $t=1, V_{1}=1, V_{2}=0$ (integrable, left), and $V_{2}=1$ (non-integrable, right) with 8359 energy levels. The spectrum is obtained with exact diagonalization by Nils Abeling. Density of states of 2510 bulk energy levels (a $p=30 \%$ fraction of all levels in the sector) is approximately constant for the integrable (middle row, left), and non-integrable case (middle row, right). Gap statistics of the bulk levels (lower row) follows Poisson statistics (blue line) in the integrable case (lower left), and $P_{\beta=1}$ GOE statistics (blue line) in the non-integrable case (lower right). For comparison, the GUE statistics $P_{\beta=2}$ (orange line) is shown (lower right). 


\subsection{Hardcore boson lattice model}

oscillator with equidistant eigenenergies and an initial state occupying $N$ consecutive eigenstates uniformly. Note that the coefficients (5.32) are uniform if the spectrum is equidistant. For general quantum systems, without equidistant spectrum, Ref. [183] shows that the Margolus-Levitin state approximately saturate the quantum speed limit bound. Intuitively, the choice of 5.32 tends to make the local density of states $\rho(E)=\sum_{n}\left|c_{n}\right|^{2} \delta\left(E-E_{n}\right)$ as uniform as possible in the sense that the probability $\int_{E}^{E+\Delta} \rho\left(E^{\prime}\right) d E^{\prime} \approx \Delta$ const is approximately constant as a function of $E$ on scales $\Delta$ larger than the mean energy spacing. At energies, for which the local spectral density of states is low, i.e. when the energy gaps are large, the weights $\left|c_{n}\right|^{2}$ are proportionally larger to compensate for this. Also note, if some energies are $n$-fold degenerate, i.e. $E_{i+1}=\cdots=E_{i+n}$, the MargolusLevitin state will only occupy a one-dimensional ray in the $n$-dimensional eigenspace. Which ray becomes occupied is not fixed by the definition, but is irrelevant for the entropy measures studied below.

We discuss the bipartite entanglement of the history state $|\Psi\rangle=\frac{1}{\sqrt{c}} \int_{0}^{c}\left|\psi_{t}\right\rangle|t\rangle d t$ as measured by the entropy of the reduced density matrix $\operatorname{Tr}_{T}|\Psi\rangle\langle\Psi|$. The main results are summarized in Figs. 5.9 and 5.10 .

On the one hand, according to the discussion leading to Eq. (5.30), the spectral gap statistics determines the behavior of the second Rényi entropy $S_{2}(c)$ for $c \geq t_{H}$. Figure 5.9 confirms the large $c$ scaling of $S_{2}(c)$ as predicted by the power law (5.30) for the integrable and non-integrable case. Moreover, also the von Neumann entropy (being bounded from below by the second Rényi entropy) obeys the same power law behavior for large $c$. On the other hand, for small $c$ the integrable and non-integrable situations cannot be distinguished by the behavior of the entropies $S_{\mathrm{vN}}(c)$ and $S_{2}(c)$.

Intuitively, on time intervals of size $\Delta t=c$ the quantum dynamics of $\left|\psi_{t}\right\rangle$ is only sensitive to spectral details on the scale of $1 / \Delta t$. This is a manifestation of the uncertainty principle between a quantity and its Fourier dual [171], also see Sec. 2.3. Hence, for small time window sizes $c$ the entropy $S(c)$ depends on the spectral density on a coarse scale $\sim 1 / c$, at which the integrable and non-integrable spectra are indistinguishable, cf. Fig. 5.8. As the quantum evolution unfolds with increasing $c$, more details of the spectral density can be resolved. At the Thouless time scale correlations between eigenvalues start to have an impact on the entropy. Finally, when $c$ is comparable to the Heisenberg time $t_{H}$, the gaps between consecutive eigenvalues are resolved, which yields the power law behavior that discriminates between integrable and non-integrable systems.

We comment on the implications of the power law

$$
S_{\mathrm{vN}}(c) \approx S_{\mathrm{vN}}(\infty)-\mathcal{A} c^{-\gamma} \quad \text { for } c \gtrsim t_{H}
$$

on the quantum clock interpretation, cf. Sec. 5.3.2. As the time window size is doubled, $c \mapsto 2 c$, Holevo's quantity increases by $\Delta \chi=S_{\mathrm{vN}}(2 c)-S_{\mathrm{vN}}(c)=\mathcal{A} c^{-\gamma}\left(1-2^{-\gamma}\right)$. There are two competing effects. On the one hand, the growth of $\chi$ entails an increase in the accessible information about the time instant within the observation time of $2 c$. On the other hand, as the observation window is doubled, an additional bit is required to maintain the accuracy on the time estimate. The additional bit is used to distinguish the two halves of the doubled time window, and the remaining bits are used to specify the time within each half. Hence, the increase of $\Delta \chi$ has to be compared to $\log 2$. If $\Delta \chi / \log 2<1$, the accessible information provided by the quantum clock is insufficient to sustain the accuracy of the time estimate as $c$ increases beyond $t_{H}$.

We compute $\Delta \chi / \log 2$ with $(5.33)$ at the Heisenberg time $c=t_{H}=2 \pi /\langle\Delta E\rangle$ when (5.33) starts to approximate $S_{\mathrm{vN}}(c)$. The numerical values are $\Delta \chi / \log 2 \approx 0.36$ and $\Delta \chi / \log 2 \approx 0.27$ for the integrable an non-integrable case of Fig. 5.8, respectively. Hence, the accuracy of the quantum 


\section{History state entanglement}

clock ceases to persist in the regime when $c$ is comparable to the Heisenberg time, irrespective of integrability and non-integrability.

In contrast, for small values of $c$ when $S_{\mathrm{vN}}(c)$ grows logarithmically one has $S_{\mathrm{vN}}(2 c) \sim S_{\mathrm{vN}}(c)+$ $\log 2$, cf. Fig. 5.9 (top row), and Eq. (5.28). Consequently, $\Delta \chi / \log 2 \sim 1$, and the precision of the quantum clock remains constant. Again, this result holds for both, integrable and non-integrable systems. The regime of logarithmic growth is valid for larger $c$ in the non-integrable situation than in the integrable case, cf. Fig. 5.9.

Finally, in Fig. 5.10 we compare the entropy for initial states with uniform energy distribution to Margolus-Levitin initial states (5.32).

In the integrable situation the power law saturation is inversely proportional for uniform initial states and inversely quadratic for Margolus-Levitin initial states. Also, the regime of logarithmic growth for small $c$ is enlarged for Margolus-Levitin states compared to uniform states. Hence, the integrable situation with Margolus-Levitin initial states becomes similar to the non-integrable situation with uniform initial states. This is plausible, because the occupation of energy levels with small gaps is suppressed in the Margolus-Levitin state, which leads to a local density of states that resembles level repulsion. It also conforms to the intuition that Margolus-Levitin states yield fast quantum dynamics.

The situation is different in the non-integrable situation, where the difference between uniform initial states and Margolus-Levitin initial states is marginal.

Remark. We have discussed the history entanglement entropy in the hardcore boson model in the integrable and fully chaotic situation. An obvious question for further investigations is how the crossover between these cases occurs, i.e. how the entanglement behaves for small integrability breaking. In view of Eq. (5.30) one may expect a power law saturation in the large $c$ limit with a fractional exponent. 

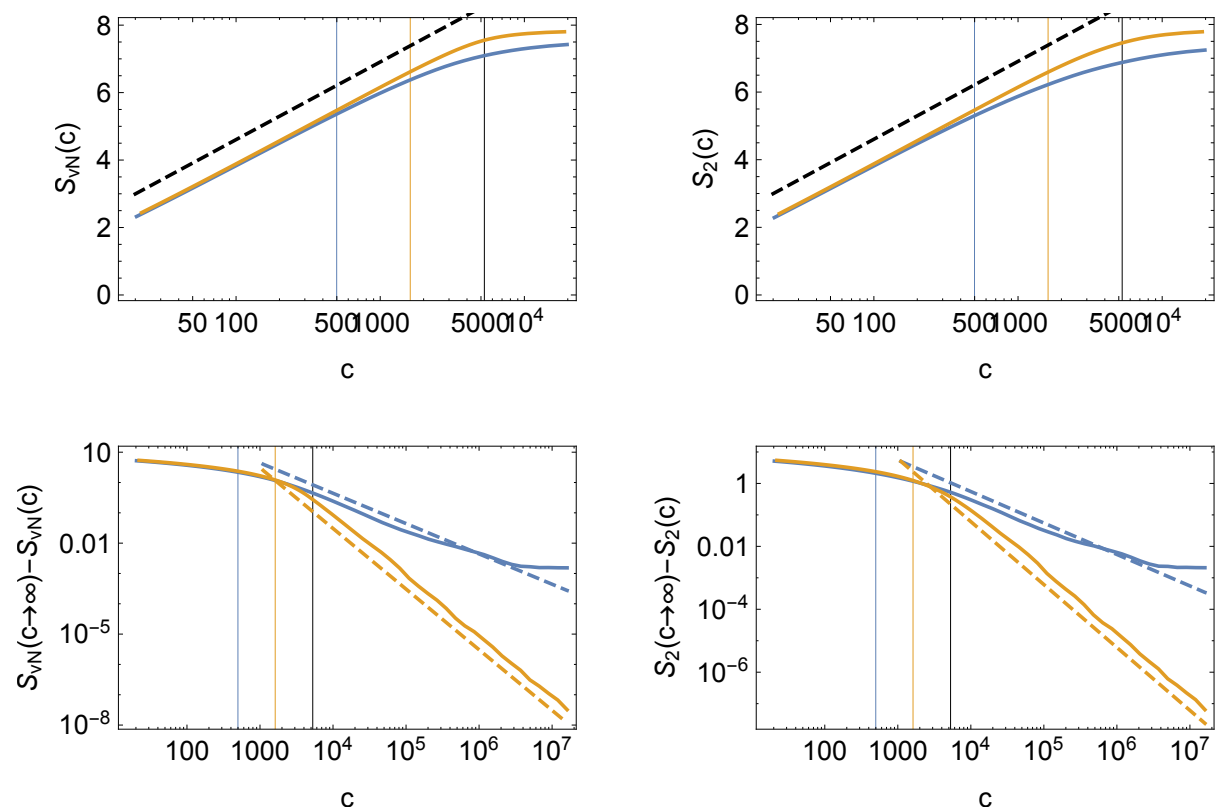

Figure 5.9: Integrable vs. non-integrable dynamics. Logarithmic growth (top) and power law saturation (bottom) of the von Neumann (left) and second Rényi (collision) entropy (right) of the history matrix as a function of the time window size $c$ in the hardcore boson model (5.31). The integrable (blue), and non-integrable (orange) situation of Fig. 5.8 are compared. In both cases (integrable and non-integrable) the initial state uniformly occupies the $p=30 \%$ bulk of the $(\widehat{k}, \widehat{P}, \widehat{Z})=(0,1,1)$ symmetry sector. For small $c$ below $2 \pi / \max \Delta E$ (vertical blue and orange line for integrable and non-integrable case, respectively) the entropies grow logarithmically (top), cf. (5.28). As $c$ becomes comparable to the mean energy spacing, i.e. the Heisenberg time $2 \pi /\langle\Delta E\rangle$ (vertical black line, identical for integrable and non-integrable case), the entropy approaches $\lim _{c \rightarrow \infty} S(c)$ inversely linear (blue), and inversely quadratic (orange) in the integrable and non-integrable case, respectively (bottom). The dashed lines, $\log c$ (dashed black), $\propto c^{-1}$ (dashed blue), and $\propto c^{-2}$ (dashed orange) are guides to the eye. 

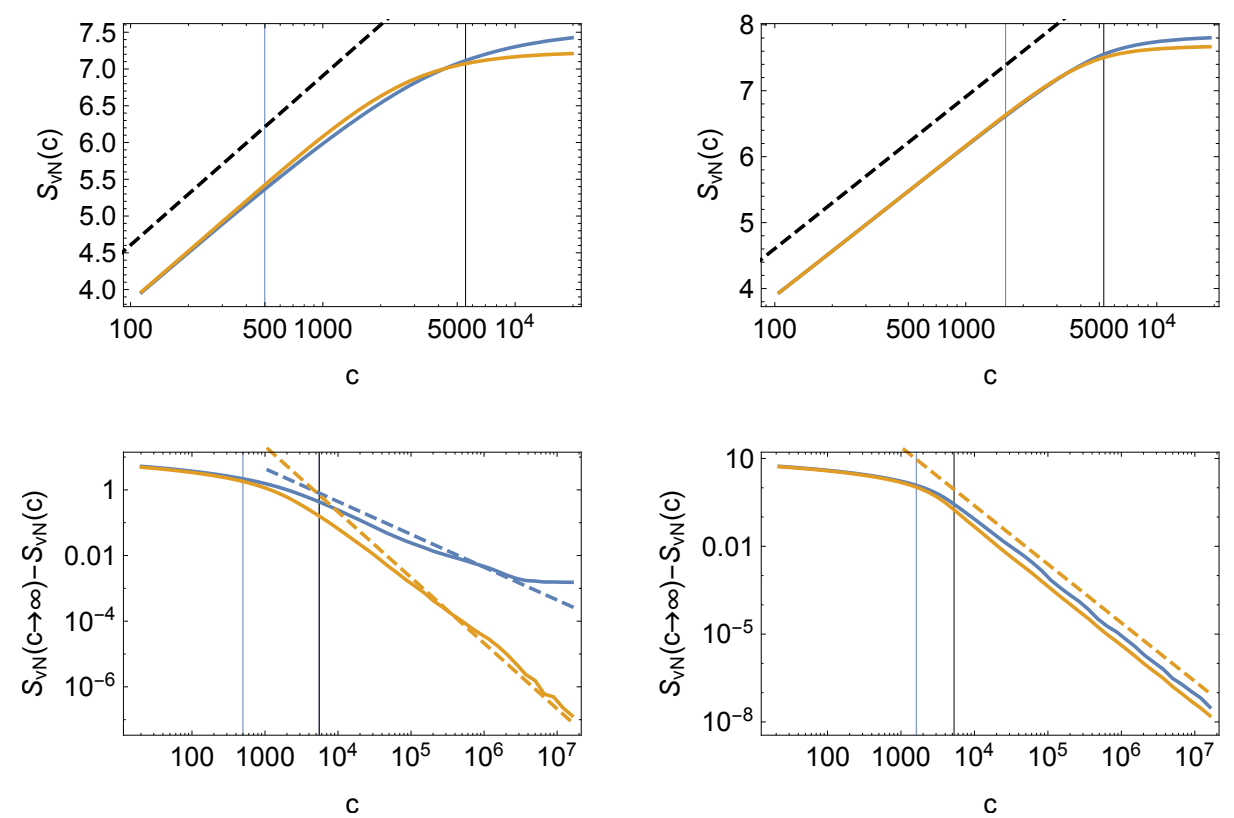

Figure 5.10: Uniform vs. Margolus-Levitin initial state. Logarithmic growth (top) and power law saturation (bottom) of the history matrix's von Neumann entropy in the integrable (left) and non-integrable situation (right) of Fig. 5.8. Initial states with uniform overlaps (blue) and Margolus-Levitin overlaps, cf. (5.32), (orange) in the $p=30 \%$ bulk are compared. Vertical lines indicate the time scales $2 \pi / \max \Delta E$ (blue) and $t_{H}=2 \pi /\langle\Delta E\rangle$ (black) proportional to the inverse max energy gap and the inverse mean energy gap (Heisenberg time). Dashed lines indicate logarithmic growth $\log c$ (dashed black), and power laws $\propto c^{-1}$ (dashed blue), $\propto c^{-2}$ (dashed orange), to guide the eye. While the uniform initial state and the Margolus-Levitin initial state yield similar entropy behavior in the non-integrable case (right), the integrable case is sensitive to uniform vs. Margolus-Levitin initial state (left). In particular, the regime of logarithmic growth holds for larger $c$ (top left), and the saturation changes to the inversely quadratic power law (bottom right) compared to the uniform initial state. 


\section{Conclusion and outlook}

Quantum many body physics out of equilibrium has lead to a plethora of novel non-thermal states of matter. The advancements of experimental techniques allows to realize and control many of them in the lab. On the one hand, the interplay of quantum entanglement and quantum complementarity promises the technological revolution of quantum computation. On the other hand, the interplay between entanglement and the exponential scaling of the Hilbert space dimension with system size makes the theoretical and numerical treatment notoriously difficult. Relevant models, in which interesting question can be addressed analytically are rare.

In this treatise we have used the transverse field Ising model of $N$ fully connected spins as a simple, yet non-trivial, model to investigate non-equilibrium dynamics analytically. Exploiting the high symmetry of permutation invariance among the spins allows to study the mean field limit by semiclassical techniques. Based on a rate function expansion, we have obtained corrections to the mean field limit that are non-perturbative in the coupling and thus provide a complementary approach to commonly used perturbative analysis of low-dimensional models. More precisely, a systematic $1 / N$ expansion of the expected magnetization, its variance, and higher cumulants after a quantum quench was derived. Remarkably, the dynamics is governed by a simple hierarchical system of ordinary differential equations in which the evolution of the cumulants depends on lower order cumulants, but not on higher order cumulants. In other words, the equations of motion of the first cumulants close, and there is no need to truncate the system.

The formalism has been applied to (i) classify different phases in the dynamical phase diagram, (ii) benchmark the validity of mean field in non-equilibrium situations, (iii) understand the growth of entanglement in different dynamical phases, and (iv) investigate the Loschmidt fidelity measure as a function of time.

To (i): Four qualitatively different dynamical phases are discriminated by the behavior of the magnetization variance. The most subtle of them is characterized by periodically enhanced spreading and squeezing. We have identified and explained a dephasing mechanism among nearby effective orbits as the driving forces behind this phase. The reasoning is independent of the fixed point structure, and only relies on the ubiquitous non-harmonic nature of the effective energy landscape. In this sense, the periodically enhanced squeezing regime is universal and expected to be found in other models, that have a semiclassical effective description.

To (ii): Even away from the unstable fixed point and the homoclinic orbit of the pitchfork bifurcation, the dephasing mechanism among nearby orbits constraints the time scale up to which mean field can be expected to be valid. More precisely, dephasing leads to a breakdown of mean field at relatively early times that scale with the square root of the system size.

To (iii): The entanglement Hamiltonian in the large $N$ limit was found to be a harmonic oscillator. A quantitative relation between entanglement and spin squeezing, and explicit expressions for the Rényi entanglement entropies were derived. The regime of periodically enhanced spin squeezing entails slow logarithmic growth of the entanglement entropy. The knowledge of the full entanglement spectrum goes beyond the mere knowledge of the entanglement entropy. Based on the entanglement spectrum and a theorem of quantum information theory, we have seen that the time evolution between two instants of time can be emulated by a protocol of local operations and classical communication (LOCC), if, and only if, the entanglement entropy is non-increasing in the course of time. 


\section{Conclusion and outlook}

To (iv): Two different arguments in favor of the existence of non-analytic points in the Loschmidt return rate as a function of time, i.e., dynamical phase transitions (DPTs), in the fully connected transverse field Ising model are given. One is based on the Liouville dynamics in the effective phase space picture. The other is based on the spectral properties of the post-quench Hamiltonian and the distribution of the excess energy after the quench. Both approaches are not mathematically rigorous. Yet, the second approach is expected to give exact results. It shows that DPT depend on the subtle details in the exponentially suppressed tails of the excess energy distribution. The quadratic decay of the Loschmidt rate function envelop is identified as a consequence of the slope of the post quench density of states.

Outlook. We close the conclusion with a brief outlook on further possible investigations. Naturally, this discussion is less concrete, and to some extent speculative.

We have seen that the dictionary between the quantum spin model and the effective semiclassical description translates results from classical physics (such as the symplectic squeezing) to genuine quantum concepts (such as entanglement). In the fully connected transverse field Ising model, which is the main matter of interest in this thesis, a site permutation invariant basis of the Dicke subspace is completely parametrized by a single good quantum number, namely the number of up spins, or, equivalently, the mean magnetization. This leads to a one-dimensional effective semiclassical description.

However, as alluded to in Secs. 3.4 and 3.7, the formalism is not limited to semiclassical dynamics in two phase space dimensions. Indeed, we have suggested to use inhomogeneous spin states in the FC-TFIM to obtain semiclassical effective models in $2 k$ dimensional phase space, where $k$ is controlled by the inhomogeneity of the spin state.

It would be interesting to work out the consequences of the rich phenomena of classical mechanics beyond one dimensions, and see what they imply for the quantum spin model. In particular, the generalization of the dephasing mechanism of nearby periodic orbits to higher phase space dimensions is expected to lead to new dynamical phases. This is because the monodromy matrix becomes higher dimensional, allowing for more classes of Jordan normal forms, cf. chapter 6 of [196]. Depending on whether the effective semiclassical description has a classically integrable or fully chaotic limit, periodic orbits are either embedded in a $(k-1)$ dimensional family of periodic orbits, or occur isolated. This distinction has influence on the monodromy matrix.

Finally, multipartite entanglement measures are used as information-theoretic, operator-independent quantities to classify unitary quantum dynamics. The quadripartite entanglement measure, known as tripartite information $I_{3}$, that quantifies how fast local information gets delocalized in the course of the evolution. However, even for simple Fermion systems, $I_{3}$ is notoriously difficult to compute [197. The large $N$ limit of the FC-TFIM provides a candidate to obtain analytical results for $I_{3}$ by the semiclassical techniques used in this thesis [197. In this respect, the higher dimensional analogs may prove to be particularly interesting, as they allow for classically chaotic limits in contrast to the trivially integrable situation. 


\section{A Star logarithm of Gaussian Wigner functions}

This appendix derives Eqs. (3.21) and (3.30) of chapter 3 . The main result are stated in Eqs. (A.4) and (A.5), from which A.6) follows as a Corollary. This result is also used in Eq. (3.21) to states the entanglement Hamiltonian of Gaussian pure states.

We consider a $2 n$ dimensional phase space with coordinates $z=(x, p) \in \mathbb{R}^{2 n}$ and $2 n$ by $2 n$ standard symplectic form $J=((0,1),(-1,0))$. For a generic Wigner function $W_{A}$ associated to a self adjoint operator $\widehat{A}$, the star-logarithm $\log ^{*}$ and the star-exponential exp* are defined through the their operator analogs via the following commuting diagram.

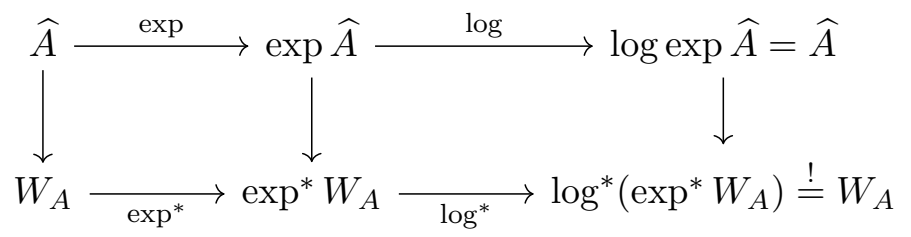

In other words, just as for the regular exponential and logarithm, the log* is the inverse of exp*. More explicitly, $\left(\exp ^{*} W\right)(z)=\sum_{k=0}^{\infty} W^{* k}(z) / k$ !, where $W^{* k}$ denotes the Moyal star-product of $k$ factors of $W$. In the special case when $\widehat{A}=-\frac{1}{2} \widehat{z} V \widehat{z}$ is quadratic in the canonical coordinates $\widehat{z}=(\widehat{x}, \widehat{p})$ with positive quadratic form $V$, the star-exponential $\exp ^{*}\left(W_{A}\right)$ is a Gaussian function and can be worked out explicitly. The calculation involves two steps.

First, if the function $W(z)=\sum_{j=1}^{n} W_{j}\left(z_{j}\right)$ is the sum of terms that depend only on $z_{j}=\left(x_{j}, p_{j}\right)$, then the star-exponential

$$
\left(\exp ^{*} W\right)(z)=\prod_{j=1}^{n} \exp ^{*} W_{j}\left(z_{j}\right)
$$

factorizes, akin to the regular exponential function 1 . In general, however, $\exp ^{*}(f+g) \neq \exp ^{*}(f) \exp ^{*}(g)$. Therefore, the star-exponential of a decoupled quadratic form $\widehat{A}=-\frac{1}{2} \sum_{j} \lambda_{j}\left(x_{j}^{2}+p_{j}^{2}\right)$ reduces to the $n=1$ dimensional case, which has been worked out in 134,143 as

$$
\left(\exp ^{*} W_{A}\right)(z)=\mathcal{C} \prod_{j} \exp \left[-\tanh \left(\frac{\lambda_{j}}{2}\right) \lambda_{j} z_{j}^{\prime 2}\right]
$$

where $\mathcal{C}^{-1}=\prod_{j} \cosh \frac{\lambda_{j}}{2}$.

Second, we apply Williamson's theorem to generalize A.2 to the situation of a general quadratic form $\widehat{A}=-\frac{1}{2} \widehat{z} V \widehat{z}$. That is, let $S \in \operatorname{Sp}(n)$ the symplectic matrix, that transforms $V$ into the normal form

$$
S^{T} V S=\operatorname{diag}(\Lambda, \Lambda), \quad \text { where } \quad \Lambda=\operatorname{diag}\left(\lambda_{1}, \cdots \lambda_{n}\right)
$$

\footnotetext{
${ }^{1}$ This is as a consequence of $f\left(z_{j}\right) * g\left(z_{k}\right)=f\left(z_{j}\right) g\left(z_{k}\right)$ for $j \neq k$, and hence $\left(W_{1}+W_{2}\right)^{* k}=\sum_{j}^{k}\left(\begin{array}{c}k \\ j\end{array}\right) W_{1}^{* j} W_{2}^{*(k-j)}$.
} 
and $\lambda_{1}, \cdots \lambda_{n}$ are the symplectic eigenvalues of $V$. Consider the following commuting diagram,

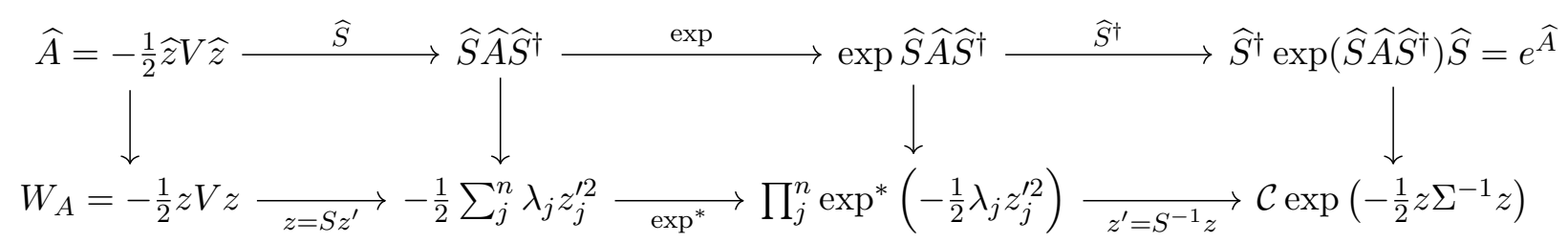

Here, $\widehat{S}$ is $\mathrm{2}^{2}$ unitary metaplectic operator associated to $S$. Interestingly, the $2 n$ by $2 n$ covariance matrix $\Sigma$ can be stated explicitly as a function of $V$ and $J$ as

$$
\Sigma^{-1}=-2 J|J V| \tan \left(\frac{1}{2} J V\right)
$$

$\left(|J V|\right.$ being the modulus of the matrix defined via the spectral theorem). Moreover, $\mathcal{C}^{-1}=$ $\prod_{j} \cosh \lambda_{j} / 2$ is a function of the symplectic spectrum $\lambda_{j}$ of $V$, and may be written as

$$
\mathcal{C}^{-1}=\sqrt{\operatorname{det} \cos \frac{J V}{2}}
$$

as a consequence of the fact that the eigenvalues of $J V$ are $\pm i \lambda_{j}$, and the symmetry of cosh. In particular, for $n=1$, A.4 yields that $\Sigma^{-1}=2 \sqrt{\operatorname{det} V} \tanh (\sqrt{\operatorname{det} V} / 2) V$ is proportional to $V$. Taking the determinant of this Eq. one can express $\operatorname{det} V$ in terms of $\operatorname{det} \Sigma$ and solve for $V=2 \sqrt{\operatorname{det} \Sigma} \operatorname{arctanh}\left[(2 \sqrt{\operatorname{det} \Sigma})^{-1}\right] \Sigma^{-1}$ as a function of $\Sigma$, cf. (3.20). In general, for $n>1$, however, $\Sigma^{-1}$ is not proportional to $V$, and it is not obvious how to solve (A.4) explicitly for $V$.

Before we derive A.4, we state the immediate consequence,

$$
\exp ^{*}\left(-\frac{1}{2} z V z\right)=\mathcal{C} \exp \left(-\frac{1}{2} z \Sigma^{-1} z\right)
$$

where $\Sigma^{-1}$ is given by A.4, and $\mathcal{C}$ is given by A.5. Thus, cf. A.1,

$$
\log ^{*} \exp \left(-\frac{1}{2} z \Sigma^{-1} z\right)=-\frac{1}{2} z V z-\log \mathcal{C} .
$$

Equations (A.6) are equivalent to 3.30 and the main result of this appendix.

We close this Sec. with a proof of (A.4). The $2 n$ by $2 n$ covariance matrix $\Sigma$ is defined through

$$
z \Sigma^{-1} z=\sum_{j}^{n} 2 \lambda_{j} \tanh \left(\lambda_{j} / 2\right) z_{j}^{\prime 2}
$$

where $z=S z^{\prime}$, cf. A.2). The right hand side can be written as $z^{\prime} g(D) z^{\prime}$, where $D=S^{T} V S$ is given by (A.3), and $g(\lambda)=2|\lambda| \tanh \lambda / 2$. Unfortunately, $g\left(S^{T} V S\right) \neq S^{T} g(V) S$, because $S^{T} \neq S^{-1}$ in general. However, we may introduce the function $f(x)=2|x| \tan x / 2$, such that ${ }^{3} g(D)=-J f(J D)$. Now, since $S$ and $S^{T}$ is symplectic, it follows that $S^{T} J=J S^{-1}$ and $J S^{T}=S^{-1} J$. We can use

\footnotetext{
${ }^{2}$ The metaplectic operator $\widehat{S}$ corresponding to $S \in \operatorname{Sp}(n)$ is only determined up to a sign. This sign ambiguity does not play a role, though, because the number of $\widehat{S}$ appearing is even.

${ }^{3}$ This trick woks for any antisymmetric function $g(\lambda)$ to which one may associate the antisymmetric function $f(x)=i g(-i x)$ by a 'Wick rotation' of the argument $\lambda \mapsto-i x$. Equivalently, $g(\lambda)=-i f(i \lambda)$, which is the scalar analog of $g(D)=-J f(J D)$, and $J$ is the matrix analog of the imaginary unit in the sense that $J^{2 n+1}=(-1)^{n} J$ and $J^{2 n}=(-1)^{n} 1$. This can also be viewed as the manifestation of the fact that the symplectic eigenvalues of $V$ are $\pm i$ times the eigenvalues of $J V$. The reason for the absolute value in the definition of $g$, is to make $g$ antisymmetric.
} 
these two identities to trade $S^{T}$ for $S^{-1}$ in order to apply the functional calculus,

$$
\begin{aligned}
g(D) & =-J f(J D)=-J f\left(J S^{T} V S\right)=-J f\left(S^{-1} J V S\right) \\
& =-J S^{-1} f(J V) S=-S^{T} J f(J V) S .
\end{aligned}
$$

Hence,

$$
z \Sigma^{-1} z=-z^{T} J f(J V) z
$$

implying the desired result (A.4). 
A Star logarithm of Gaussian Wigner functions 


\section{B Dicke state entanglement entropy}

In this appendix we compute the von Neumann entanglement entropy of a Dicke state, cf. 2.2

$$
\left|N_{+}\right\rangle=\left(\begin{array}{c}
N \\
N_{+}
\end{array}\right)^{1 / 2} P\left(|\uparrow\rangle^{\otimes N_{+}} \otimes|\downarrow\rangle^{\otimes N-N_{+}}\right)
$$

w.r.t. the bipartition $\mathcal{D}_{N}=\mathcal{D}_{A} \otimes \mathcal{D}_{B}$ of $N$ spins into two disjoint subsets $A$ and $B$ of the spins. The main result of this appendix is Eq. (B.1).

By

$$
\left|N_{+}\right\rangle=\sum_{A_{+}+B_{+}=N_{+}} \sqrt{\left(\begin{array}{c}
N_{A} \\
A_{+}
\end{array}\right)\left(\begin{array}{c}
N_{B} \\
B_{+}
\end{array}\right) /\left(\begin{array}{c}
N \\
N_{+}
\end{array}\right)}\left|A_{+}\right\rangle\left|B_{+}\right\rangle
$$

the reduced density matrix of the Dicke state $\left|N_{+}\right\rangle$w.r.t. the partition into groups $A$ and $B$ is given by

$$
\rho_{A}=\sum_{N_{A}^{+}=0}^{N_{A}} \frac{\left(\begin{array}{c}
N_{A} \\
N_{A}^{+}
\end{array}\right)\left(\begin{array}{c}
N-N_{A} \\
N^{+}-N_{A}^{+}
\end{array}\right)}{\left(\begin{array}{c}
N \\
N^{+}
\end{array}\right)}\left|N_{A}^{+}\right\rangle\left\langle N_{A}^{+}\right|,
$$

i.e. it is diagonal in the Dicke basis of subsystem $A$ with eigenvalues $\Lambda\left(N_{A}^{+}\right)=\left(\begin{array}{c}N_{A} \\ N_{A}^{+}\end{array}\right)\left(\begin{array}{c}N-N_{A} \\ N^{+}-N_{A}^{+}\end{array}\right) /\left(\begin{array}{c}N \\ N^{+}\end{array}\right)$. The $q$ th Rényi entanglement entropy

$$
S_{q}\left(N^{+}\right)=\frac{1}{1-q} \log \sum_{N_{A}^{+}} \Lambda\left(N_{A}^{+}\right)^{q}
$$

of this Dicke state can be calculated to leading order in $N$ by approximating the eigenvalues $\Lambda\left(N_{A}^{+}\right)$ of $\rho_{A}$ using Stirling's formula and approximating the (Riemann) sum by an integral. The final result is

$$
S_{q}\left(N^{+}\right) \stackrel{N^{+} / N=n^{+}=\text {const }}{\longrightarrow} \frac{1}{q-1} \log \sqrt{q}+\frac{1}{2} \log \left[N 2 \pi \alpha \beta n^{+}\left(1-n^{+}\right)\right],
$$

where $\alpha=A / N$ and $\beta=B / N$ are the relative subsystem sizes. Interestingly, apart from an additive term, this result is independent of $q$. As $q \rightarrow 1$ (von Neumann entropy) the first term becomes $1 / 2$. Note that the above result is only valid for $n^{+},\left(1-n^{+}\right)>\mathcal{O}(1 / N)$.

Proof. We want to compute $\operatorname{Tr} \rho_{A}^{q}=\sum_{N_{A}^{+}} \Lambda\left(N_{A}^{+}\right)^{q}$. For $N \gg 1, \Lambda\left(N_{A}^{+}\right) \sim \exp \left(-N S_{\alpha}\left(n_{A}^{+}, n_{B}^{+}\right)\right)$ is of large deviation form with rate function $S_{\alpha}\left(n_{A}^{+}, n_{B}^{+}\right)=\alpha D\left(n_{A}^{+} \| n^{+}\right)+\bar{\alpha} D\left(n_{B}^{+} \| n^{+}\right)$, where $\alpha=N_{A} / N, n_{A, B}^{+}=N_{A, B}^{+} / N_{A, B}$ and $n^{+}=N^{+} / N$ such that $n^{+}=\alpha n_{A}^{+}+\beta n_{B}^{+}$, and $D\left(n_{1} \| n_{2}\right)$ is the relative entropy of two Bernoulli random variables with success probability $n_{1}$ and $n_{2}$, respectively. Because $\Lambda\left(N_{A}^{+}\right)$is a slowly varying function (it varies on the scale $\sqrt{N}$ ), we may replace the summation $\sum_{N_{A}^{+}}$by the integral $N_{A} \int_{0}^{1} d n_{a}^{+}$. Hence,

$$
\operatorname{Tr}\left(\rho_{A}^{q}\right) \sim N_{A} \int_{0}^{1} d n_{A}^{+} e^{-q N S_{\alpha}\left(n_{A}^{+}, n_{B}^{+}\right)} .
$$

The integral is computed with the saddle point approximation. The unique dominating saddle 


\section{$B$ Dicke state entanglement entropy}

point is at $n_{A}^{+}=n_{B}^{+}=n^{+}$and the second derivative of $S_{\alpha}\left(n_{A}^{+}, n_{B}^{+}\right)$w.r.t. $n_{A}^{+}$at $n^{+}$is given by $\frac{\alpha}{\beta}\left(n^{+}\left(1-n^{+}\right)\right)^{-1}$ (this is $\frac{\alpha}{\beta}$ times the Fisher information of a Bernoulli random variable with success probability $n^{+}$). We get,

$$
\operatorname{Tr} \rho_{A}^{q} \sim \sqrt{N 2 \pi \alpha \beta n^{+}\left(1-n^{+}\right) / q}
$$

and thus,

$$
S_{q}=\frac{1}{1-q} \log \frac{\operatorname{Tr} \rho_{A}^{q}}{\left(\operatorname{Tr} \rho_{A}\right)^{q}} \rightarrow \frac{1}{q-1} \log \sqrt{q}+\frac{1}{2} \log \left(N 2 \pi \alpha \bar{\alpha} n^{+}\left(1-n^{+}\right)\right)
$$




\section{Dynamics of nearby orbit variance in higher dimensions}

This appendix derives Eq. (C.2), the generalization of (3.13a) for higher dimensional phase spaces. The result is used in 3.46 of Sec. 3.7.

The main difficulty in generalizing the result (3.13) to higher dimensions, is solved by an efficient notation. The Hamiltonian $H_{\mathrm{eff}}: \mathbb{R}^{2 k} \rightarrow \mathbb{R}$ is a real-valued function on $2 k$-dimensional phase space, and the rate function $f: \mathbb{R}^{k} \rightarrow \mathbb{C}$ is a complex-valued function on the $k$-dimensional configuration space (to ease the notation, we drop eff off the Hamiltonian and write $H$ for $H_{\text {eff }}$ ). We write indices related to positions as superscripts, such as in $x^{j}$, and indices related to momenta as subscripts, such as in $p_{j}$. Derivatives of a quantity $Q$ w.r.t. $x^{m}$ and $p_{l}$ are denoted by preceding the index $m$ and $l$ with a colon, e.g. $\partial_{x^{m}} \partial_{x^{n}} \partial_{p_{l}} Q=Q_{, m n}^{, l}$. Note that derivatives w.r.t. positions appear as subscripts, while derivatives w.r.t. momenta as superscripts. This indicates that derivatives transform differently, and keeps the indices in the equations of motion

$$
\dot{\mathbf{x}}^{j}=H^{, j}(\mathbf{x}, \mathbf{p}), \quad \text { and } \quad \dot{\mathbf{p}}_{j}=-H_{, j}(\mathbf{x}, \mathbf{p})
$$

balanced.

Analogously to (3.5), the rate function is expanded around the non-degenerate minimum of $\Re f$ at $\mathbf{x}=\mathbf{x}_{\mathrm{cl}}$

$$
f(\mathbf{x})=\sum_{j=0} \frac{1}{j !} f_{(j)}\left(\mathbf{x}-\mathbf{x}_{\mathrm{cl}}\right)^{j},
$$

where $f_{(j)}=\left.\nabla_{\mathbf{x}}^{j} f(\mathbf{x})\right|_{\mathbf{x}_{\mathrm{cl}}}$ is a symmetric tensor with $j$ lower indices, and $f_{(j)}\left(\mathbf{x}-\mathbf{x}_{\mathrm{cl}}\right)^{j}$ denotes the contraction of $f_{(j)}$ with $j$ factors of the rank one tensor $\left(\mathbf{x}-\mathbf{x}_{\mathrm{cl}}\right)$. Analogously to 3.12 one has

$$
\dot{f}_{(j)}=\left.i \nabla_{\mathbf{x}}^{j} H\left[\mathbf{x}, i \nabla_{\mathbf{x}} f(\mathbf{x})\right]\right|_{\mathbf{x}_{\mathrm{cl}}}+f_{(j+1)} \cdot \dot{\mathbf{x}}_{\mathrm{cl}},
$$

where the dot in the last term denotes the contraction between the symmetric rank $j+1$ tensor $f_{(j+1)}$ and the rank one tensor $\dot{\mathbf{x}}_{\mathrm{cl}}$. In particular, for $f_{(1)}$, by the chain rule

$$
\left(\dot{f}_{(1)}\right)_{j}=i H_{, j}-H^{, l} f_{, l j}+\left(f_{(2)} \cdot \dot{\mathbf{x}}_{\mathrm{cl}}\right)_{j}
$$

(summation over repeated indices is implicit, the convention on the placement of indices guarantees that repeated indices automatically appear once as a superscript and once as a subscript), where $H$ is evaluated at $\left(\mathbf{x}_{\mathrm{cl}}, i f_{(1)}\right)$ and $f$ is evaluated at $\mathbf{x}=\mathbf{x}_{\mathrm{cl}}$. Considering real and imaginary part of this equation separately, using that $\Re f_{(2)}$ is non-degenerate, and identifying $i f_{(1)}=\mathbf{p}_{\mathrm{cl}} \in \mathbb{R}$, yields the equations of motion (C.1) for $\left(\mathbf{x}_{\mathrm{cl}}, \mathbf{p}_{\mathrm{cl}}\right)$, independent of $f_{(2)}$. For the Hessian $f_{(2)}$ of $f$ one has

$$
\left(\dot{f}_{(2)}\right)_{m n}=\left[i H_{, m n}-H_{, m}^{, j} f_{, j n}-H_{, n}^{, j} f_{, j m}-i H^{, j l} f_{, j m} f_{, l n}-H^{, j} f_{, j m n}\right]_{\mathbf{x}_{\mathrm{cl}}}+\left(f_{(3)} \cdot \dot{x}_{\mathrm{cl}}\right)_{m n},
$$

which is independent of $f_{(3)}$, since the last two terms cancel by (C.1). The last equation is equivalent 
C Dynamics of nearby orbit variance in higher dimensions

to

$$
\begin{aligned}
\dot{X}_{m n} & =-H_{, m}^{, j} X_{j n}-H_{,,}^{, j} X_{j m}+H^{, j l}\left(X_{j m} Y_{l n}+Y_{j m} X_{l n}\right) \quad \text { and } \\
\dot{Y}_{m n} & =H_{, m n}-H_{, m}^{, j} Y_{j n}-H_{, n}^{, j} Y_{j m}-H^{, j l}\left(X_{j m} X_{l n}-Y_{j m} Y_{l n}\right)
\end{aligned}
$$

upon writing the rank two tensor $f_{(2)}=X+i Y$ as a $k$ by $k$ matrix with real part $X$ and imaginary part $Y$.

Similar to Eq. C.2 , one can obtain the flow of the higher order expansion coefficients $f_{(j)}$ for $j>2$. These coefficients can then be used to compute higher order moments (and cumulants) beyond the variance, cf. Sec. 3.2 . 


\section{Bibliography}

[1] I. Homrighausen and S. Kehrein, Out of equilibrium mean field dynamics in the transverse field Ising model, arXiv e-prints arXiv:1908.02596 (2019), 1908.02596.

[2] I. Homrighausen, N. O. Abeling, V. Zauner-Stauber and J. C. Halimeh, Anomalous dynamical phase in quantum spin chains with long-range interactions, Phys. Rev. B 96, 104436 (2017), doi:10.1103/PhysRevB.96.104436.

[3] K. Zuse, Rechnender Raum, Schriften zur Datenverarbeitung. Vieweg, ISBN 978-3-66300810-1, doi:10.1007/978-3-663-02723-2 (1969).

[4] E. Fredkin, An introduction to digital philosophy, International Journal of Theoretical Physics 42(2), 189 (2003), doi:10.1023/A:1024443232206.

[5] W. THOMSON, Kinetic theory of the dissipation of energy, Nature 9(232), 441 (1874), doi:10.1038/009441c0.

[6] M. Smoluchowski, Experimentell nachweisbare, der üblichen thermodynamik widersprechende molekularphänomene, Physikalische Zeitschrift 13, 1068 (1912).

[7] L. Szilard, über die entropieverminderung in einem thermodynamischen system bei eingriffen intelligenter wesen, Zeitschrift für Physik 53(11), 840 (1929), doi:10.1007/BF01341281.

[8] R. Feynman, R. Leighton and M. Sands, The Feynman Lectures on Physics, Vol. I: The New Millennium Edition: Mainly Mechanics, Radiation, and Heat, The Feynman Lectures on Physics. Basic Books, ISBN 9780465024933 (2011).

[9] R. Landauer, Information is physical, Physics Today 44, 23 (1991), doi:https://doi.org/10. $1063 / 1.881299$.

[10] S. Toyabe, T. Sagawa, M. Ueda, E. Muneyuki and M. Sano, Experimental demonstration of information-to-energy conversion and validation of the generalized jarzynski equality, Nature Physics 6(12), 988 (2010), doi:10.1038/nphys1821.

[11] S. Lloyd and Y. J. Ng, Black hole computers, SA Special Editions 17, 82 (2012), doi: doi:10.1038/scientificamerican0407-82sp.

[12] E. Schrödinger, Discussion of probability relations between separated systems, Mathematical Proceedings of the Cambridge Philosophical Society 31, 555 (1935), doi:10.1017/ S0305004100013554.

[13] A. Einstein, B. Podolsky and N. Rosen, Can quantum-mechanical description of physical reality be considered complete?, Phys. Rev. 47, 777 (1935), doi:10.1103/PhysRev.47.777.

[14] L. Bombelli, R. K. Koul, J. Lee and R. D. Sorkin, Quantum source of entropy for black holes, Phys. Rev. D 34, 373 (1986), doi:10.1103/PhysRevD.34.373.

[15] M. Srednicki, Entropy and area, Phys. Rev. Lett. 71, 666 (1993), doi:10.1103/PhysRevLett. 71.666 . 
Bibliography

[16] C. Callan and F. Wilczek, On geometric entropy, Physics Letters B 333(1), 55 (1994), doi:https://doi.org/10.1016/0370-2693(94)91007-3.

[17] J. Maldacena, Theoretical physics: Black holes and wormholes and the secrets of quantum spacetime, Scientific American 315(5), 26 (2016), doi:https://doi.org/10.1038/ scientificamerican1116-26.

[18] S. Ryu and T. Takayanagi, Holographic derivation of entanglement entropy from the antide sitter space/conformal field theory correspondence, Phys. Rev. Lett. 96, 181602 (2006), doi:10.1103/PhysRevLett.96.181602.

[19] T. Nishioka, S. Ryu and T. Takayanagi, Holographic entanglement entropy: an overview, Journal of Physics A: Mathematical and Theoretical 42(50), 504008 (2009), doi:10.1088/ $1751-8113 / 42 / 50 / 504008$.

[20] C. Holzhey, F. Larsen and F. Wilczek, Geometric and renormalized entropy in conformal field theory, Nuclear Physics B 424(3), 443 (1994), doi:https://doi.org/10.1016/0550-3213(94) 90402-2.

[21] P. Calabrese and J. Cardy, Entanglement entropy and quantum field theory, Journal of Statistical Mechanics: Theory and Experiment 2004(06), P06002 (2004), doi: 10.1088/1742-5468/2004/06/p06002.

[22] J. L. Cardya, Entanglement entropy in extended quantum systems, The European Physical Journal B 64(3), 321 (2008), doi:10.1140/epjb/e2008-00102-5.

[23] P. Calabrese and J. Cardy, Entanglement entropy and conformal field theory, Journal of Physics A: Mathematical and Theoretical 42(50), 504005 (2009), doi:10.1088/1751-8113/ $42 / 50 / 504005$.

[24] C. H. Bennett, H. J. Bernstein, S. Popescu and B. Schumacher, Concentrating partial entanglement by local operations, Phys. Rev. A 53, 2046 (1996), doi:10.1103/PhysRevA.53.2046.

[25] M. Nielsen and I. Chuang, Quantum Computation and Quantum Information: 10th Anniversary Edition, Cambridge University Press, ISBN 9781139495486 (2010).

[26] J. Eisert, M. Cramer and M. B. Plenio, Colloquium: Area laws for the entanglement entropy, Rev. Mod. Phys. 82, 277 (2010), doi:10.1103/RevModPhys.82.277.

[27] T. Barthel, S. Dusuel and J. Vidal, Entanglement entropy beyond the free case, Phys. Rev. Lett. 97, 220402 (2006), doi:10.1103/PhysRevLett.97.220402.

[28] M. Filippone, S. Dusuel and J. Vidal, Quantum phase transitions in fully connected spin models: An entanglement perspective, Phys. Rev. A 83, 022327 (2011), doi: 10.1103/PhysRevA.83.022327.

[29] V. Giovannetti, S. Lloyd and L. Maccone, Quantum-enhanced measurements: Beating the standard quantum limit, Science 306(5700), 1330 (2004), doi:10.1126/science.1104149, http: //science.sciencemag.org/content/306/5700/1330.full.pdf.

[30] J. Ma, X. Wang, C. Sun and F. Nori, Quantum spin squeezing, Physics Reports 509(2), 89 (2011), doi:https://doi.org/10.1016/j.physrep.2011.08.003. 
[31] T. Fösel, P. Tighineanu, T. Weiss and F. Marquardt, Reinforcement learning with neural networks for quantum feedback, Phys. Rev. X 8, 031084 (2018), doi:10.1103/PhysRevX.8. 031084 .

[32] S. R. White, Density matrix formulation for quantum renormalization groups, Phys. Rev. Lett. 69, 2863 (1992), doi:10.1103/PhysRevLett.69.2863.

[33] A. J. Daley, C. Kollath, U. Schollwöck and G. Vidal, Time-dependent density-matrix renormalization-group using adaptive effective hilbert spaces, Journal of Statistical Mechanics: Theory and Experiment 2004(04), P04005 (2004), doi:10.1088/1742-5468/2004/04/p04005.

[34] U. Schollwöck, The density-matrix renormalization group, Rev. Mod. Phys. 77, 259 (2005), doi:10.1103/RevModPhys.77.259.

[35] U. Schollwöck, The density-matrix renormalization group in the age of matrix product states, Annals of Physics 326(1), 96 (2011), doi:https://doi.org/10.1016/j.aop.2010.09.012, January 2011 Special Issue.

[36] S. Paeckel, T. Köhler, A. Swoboda, S. R. Manmana, U. Schollwöck and C. Hubig, Timeevolution methods for matrix-product states, Annals of Physics 411, 167998 (2019), doi: https://doi.org/10.1016/j.aop.2019.167998.

[37] L. Vidmar and M. Rigol, Entanglement entropy of eigenstates of quantum chaotic hamiltonians, Phys. Rev. Lett. 119, 220603 (2017), doi:10.1103/PhysRevLett.119.220603.

[38] M. H. Freedman, A. Y. Kitaev, M. J. Larsen and Z. Wang, Topological quantum computation, Bull. Amer. Math. Soc. 40, 31 (2003), doi:https://doi.org/10.1090/S0273-0979-02-00964-3.

[39] M. Bukov, A. G. R. Day, D. Sels, P. Weinberg, A. Polkovnikov and P. Mehta, Reinforcement learning in different phases of quantum control, Phys. Rev. X 8, 031086 (2018), doi:10.1103/ PhysRevX.8.031086.

[40] F. Arute, K. Arya, R. Babbush, D. Bacon, J. C. Bardin, R. Barends, R. Biswas, S. Boixo, F. G. S. L. Brandao, D. A. Buell, B. Burkett, Y. Chen et al., Quantum supremacy using a programmable superconducting processor, Nature 574(7779), 505 (2019), doi:10.1038/ s41586-019-1666-5.

[41] N. Wiener, Cybernetics, Second Edition: Or the Control and Communication in the Animal and the Machine, The MIT Press, ISBN 026273009X (1965).

[42] Y. Manin, Computable and uncomputable, Sovetskoye Radio (1950).

[43] R. P. Feynman, Simulating physics with computers, International Journal of Theoretical Physics 21(6), 467 (1982), doi:10.1007/BF02650179.

[44] S. Lloyd, Universal quantum simulators, Science 273(5278), 1073 (1996), doi: 10.1126/science.273.5278.1073, https://science.sciencemag.org/content/273/5278/ 1073.full.pdf.

[45] E. P. Wigner, Characteristic vectors of bordered matrices with infinite dimensions, Annals of Mathematics 62(3), 548 (1955).

[46] M. Albiez, R. Gati, J. Fölling, S. Hunsmann, M. Cristiani and M. K. Oberthaler, Direct observation of tunneling and nonlinear self-trapping in a single bosonic josephson junction, Phys. Rev. Lett. 95, 010402 (2005), doi:10.1103/PhysRevLett.95.010402. 
Bibliography

[47] J. Estève, C. Gross, A. Weller, S. Giovanazzi and M. K. Oberthaler, Squeezing and entanglement in a bose-einstein condensate, Nature 455, 1216 EP (2008), doi:10.1038/nature07332.

[48] Q. Y. He, M. D. Reid, T. G. Vaughan, C. Gross, M. Oberthaler and P. D. Drummond, Einstein-podolsky-rosen entanglement strategies in two-well bose-einstein condensates, Phys. Rev. Lett. 106, 120405 (2011), doi:10.1103/PhysRevLett.106.120405.

[49] S. Kolkowitz, S. L. Bromley, T. Bothwell, M. L. Wall, G. E. Marti, A. P. Koller, X. Zhang, A. M. Rey and J. Ye, Spin-orbit-coupled fermions in an optical lattice clock, Nature 542(7639), 66 (2017), doi:10.1038/nature20811.

[50] M. Srednicki, Chaos and quantum thermalization, Phys. Rev. E 50, 888 (1994), doi: 10.1103/PhysRevE.50.888.

[51] M. Moeckel and S. Kehrein, Crossover from adiabatic to sudden interaction quenches in the hubbard model: prethermalization and non-equilibrium dynamics, New Journal of Physics 12(5), 055016 (2010), doi:10.1088/1367-2630/12/5/055016.

[52] F. R. A. Biebl and S. Kehrein, Thermalization rates in the one-dimensional hubbard model with next-to-nearest neighbor hopping, Phys. Rev. B 95, 104304 (2017), doi: 10.1103/PhysRevB.95.104304.

[53] M. Schmitt and S. Kehrein, Effective time reversal and echo dynamics in the transverse field ising model, EPL (Europhysics Letters) 115(5), 50001 (2016), doi:10.1209/0295-5075/115/ 50001.

[54] M. Schmitt and S. Kehrein, Irreversible dynamics in quantum many-body systems, Phys. Rev. B 98, 180301 (2018), doi:10.1103/PhysRevB.98.180301.

[55] M. Serbyn, Z. Papić and D. A. Abanin, Universal slow growth of entanglement in interacting strongly disordered systems, Phys. Rev. Lett. 110, 260601 (2013), doi:10.1103/PhysRevLett. 110.260601 .

[56] D. A. Huse, R. Nandkishore and V. Oganesyan, Phenomenology of fully many-body-localized systems, Phys. Rev. B 90, 174202 (2014), doi:10.1103/PhysRevB.90.174202.

[57] C. J. Turner, A. A. Michailidis, D. A. Abanin, M. Serbyn and Z. Papic, Weak ergodicity breaking from quantum many-body scars, Nature Physics 14(7), 745 (2018), doi:10.1038/ s41567-018-0137-5.

[58] W. W. Ho, S. Choi, H. Pichler and M. D. Lukin, Periodic orbits, entanglement, and quantum many-body scars in constrained models: Matrix product state approach, Phys. Rev. Lett. 122, 040603 (2019), doi:10.1103/PhysRevLett.122.040603.

[59] M. Heyl, A. Polkovnikov and S. Kehrein, Dynamical quantum phase transitions in the transverse-field ising model, Phys. Rev. Lett. 110, 135704 (2013), doi:10.1103/PhysRevLett. 110.135704 .

[60] B. Sciolla and G. Biroli, Quantum quenches, dynamical transitions, and off-equilibrium quantum criticality, Phys. Rev. B 88, 201110 (2013), doi:10.1103/PhysRevB.88.201110.

[61] F. Wilczek, Quantum time crystals, Phys. Rev. Lett. 109, 160401 (2012), doi:10.1103/ PhysRevLett.109.160401. 
[62] H. Lipkin, N. Meshkov and A. Glick, Validity of many-body approximation methods for a solvable model: (i). exact solutions and perturbation theory, Nuclear Physics 62(2), 188 (1965), doi:http://dx.doi.org/10.1016/0029-5582(65)90862-X.

[63] M. Jääskeläinen and P. Meystre, Coherence dynamics of two-mode condensates in asymmetric potentials, Phys. Rev. A 73, 013602 (2006), doi:10.1103/PhysRevA.73.013602.

[64] V. S. Shchesnovich and M. Trippenbach, Fock-space wkb method for the boson josephson model describing a bose-einstein condensate trapped in a double-well potential, Phys. Rev. A 78, 023611 (2008), doi:10.1103/PhysRevA.78.023611.

[65] C. Bodet, J. Estève, M. K. Oberthaler and T. Gasenzer, Two-mode bose gas: Beyond classical squeezing, Phys. Rev. A 81, 063605 (2010), doi:10.1103/PhysRevA.81.063605.

[66] M. B. Hastings, Entropy and entanglement in quantum ground states, Phys. Rev. B 76, 035114 (2007), doi:10.1103/PhysRevB.76.035114.

[67] J. Eisert, M. van den Worm, S. R. Manmana and M. Kastner, Breakdown of quasilocality in long-range quantum lattice models, Phys. Rev. Lett. 111, 260401 (2013), doi:10.1103/ PhysRevLett.111.260401.

[68] Z.-X. Gong, M. Foss-Feig, S. Michalakis and A. V. Gorshkov, Persistence of locality in systems with power-law interactions, Phys. Rev. Lett. 113, 030602 (2014), doi:10.1103/ PhysRevLett.113.030602.

[69] L. Cevolani, G. Carleo and L. Sanchez-Palencia, Protected quasilocality in quantum systems with long-range interactions, Phys. Rev. A 92, 041603 (2015), doi:10.1103/PhysRevA.92. 041603.

[70] J. Schachenmayer, B. P. Lanyon, C. F. Roos and A. J. Daley, Entanglement growth in quench dynamics with variable range interactions, Phys. Rev. X 3, 031015 (2013), doi: 10.1103/PhysRevX.3.031015.

[71] M. G. Nezhadhaghighi and M. A. Rajabpour, Entanglement dynamics in short- and longrange harmonic oscillators, Phys. Rev. B 90, 205438 (2014), doi:10.1103/PhysRevB.90. 205438.

[72] A. S. Buyskikh, M. Fagotti, J. Schachenmayer, F. Essler and A. J. Daley, Entanglement growth and correlation spreading with variable-range interactions in spin and fermionic tunneling models, Phys. Rev. A 93, 053620 (2016), doi:10.1103/PhysRevA.93.053620.

[73] R. Singh, R. Moessner and D. Roy, Effect of long-range hopping and interactions on entanglement dynamics and many-body localization, Phys. Rev. B 95, 094205 (2017), doi: 10.1103/PhysRevB.95.094205.

[74] Z.-X. Gong, M. Foss-Feig, F. G. S. L. Brandao and A. V. Gorshkov, Entanglement area laws for long-range interacting systems, Phys. Rev. Lett. 119, 050501 (2017), doi:10.1103/ PhysRevLett.119.050501.

[75] A. Lerose and S. Pappalardi, Origin of the slow growth of entanglement entropy in long-range interacting systems, arXiv e-prints arXiv:1811.05505 (2018), 1811.05505.

[76] E. H. Lieb and D. W. Robinson, The finite group velocity of quantum spin systems, Communications in Mathematical Physics 28(3), 251 (1972), doi:10.1007/BF01645779. 
[77] H. Kim and D. A. Huse, Ballistic spreading of entanglement in a diffusive nonintegrable system, Phys. Rev. Lett. 111, 127205 (2013), doi:10.1103/PhysRevLett.111.127205.

[78] J. Vidal, G. Palacios and C. Aslangul, Entanglement dynamics in the lipkin-meshkov-glick model, Phys. Rev. A 70, 062304 (2004), doi:10.1103/PhysRevA.70.062304.

[79] K. R. A. Hazzard, M. van den Worm, M. Foss-Feig, S. R. Manmana, E. G. Dalla Torre, T. Pfau, M. Kastner and A. M. Rey, Quantum correlations and entanglement in far-fromequilibrium spin systems, Phys. Rev. A 90, 063622 (2014), doi:10.1103/PhysRevA.90.063622.

[80] S. Pappalardi, A. Russomanno, B. Žunkovič, F. Iemini, A. Silva and R. Fazio, Scrambling and entanglement spreading in long-range spin chains, Phys. Rev. B 98, 134303 (2018), doi:10.1103/PhysRevB.98.134303.

[81] B. Sciolla and G. Biroli, Dynamical transitions and quantum quenches in mean-field models, Journal of Statistical Mechanics: Theory and Experiment 2011(11), P11003 (2011), doi: 10.1088/1742-5468/2011/11/p11003.

[82] P. Dirac, The principles of quantum mechanics, Clarendon press, ISBN 9780198520115 (1992).

[83] L. Erdos, Lecture Notes on Quantum Brownian Motion, arXiv e-prints arXiv:1009.0843 (2010), 1009.0843.

[84] M. V. Berry, Chaos and the semiclassical limit of quantum mechanics (is the moon there when somebody looks?), In R. Russell, C. for Theology, the Natural Sciences and S. vaticana, eds., Quantum Mechanics: Scientific Perspectives on Divine Action, Quantum Mechanics, pp. 41-54. Vatican Observatory, ISBN 9780268039783 (2001).

[85] M. V. Berry and J. Goldberg, Renormalisation of curlicues, Nonlinearity 1(1), 1 (1988), doi:10.1088/0951-7715/1/1/001.

[86] W.-M. Zhang, D. H. Feng and R. Gilmore, Coherent states: Theory and some applications, Rev. Mod. Phys. 62, 867 (1990), doi:10.1103/RevModPhys.62.867.

[87] R. O'Connell and E. Wigner, Some properties of a non-negative quantum-mechanical distribution function, Physics Letters A 85(3), 121 (1981), doi:https://doi.org/10.1016/ 0375-9601(81)90881-1.

[88] A. Polkovnikov, Phase space representation of quantum dynamics, Annals of Physics 325(8), 1790 (2010), doi:https://doi.org/10.1016/j.aop.2010.02.006.

[89] E. J. Heller, Time-dependent approach to semiclassical dynamics, The Journal of Chemical Physics 62(4), 1544 (1975), doi:http://dx.doi.org/10.1063/1.430620.

[90] R. G. Littlejohn, The semiclassical evolution of wave packets, Physics Reports 138(4-5), 193 (1986), doi:http://dx.doi.org/10.1016/0370-1573(86)90103-1.

[91] E. Heller, Wavepacket dynamics and quantum chaology, In M.-J. Giannoni, A. Voros and J. Zinn-Justin, eds., Chaos and Quantum Physics, Les Houches Lecture Series LII. NorthHolland, Amsterdam (1991).

[92] M. V. Berry, Structures in semiclassical spectra: A question of scale, In S. Diner, D. Fargue, G. Lochak and F. Selleri, eds., The Wave-Particle Dualism: A Tribute to Louis de Broglie on his 90th Birthday, pp. 231-252. Springer Netherlands, Dordrecht, ISBN 978-94-009-6286-6, doi:10.1007/978-94-009-6286-6_12 (1984). 
[93] M. Berry, Random renormalization in the semiclassical long-time limit of a precessing spin, Physica D: Nonlinear Phenomena 33(1), 26 (1988), doi:https://doi.org/10.1016/ S0167-2789(98)90006-9.

[94] M. V. Berry, Regular and irregular semiclassical wavefunctions, Journal of Physics A: Mathematical and General 10(12), 2083 (1977), doi:10.1088/0305-4470/10/12/016.

[95] M. V. Berry, Semiclassical mechanics of regular and irregular motion, In G. Iooss, R. H. G. Helleman and R. Stora, eds., Les Houches Lecture Series Session XXXVI, Les Houches Summer School Proceedings Series, pp. 171-271. North-Holland Publishing Company, Amsterdam (1983).

[96] M. V. Berry and J. M. Ziman, Semi-classical mechanics in phase space: A study of wigner's function, Philosophical Transactions of the Royal Society of London. Series A, Mathematical and Physical Sciences 287(1343), 237 (1977), doi:10.1098/rsta.1977.0145, https://royalsocietypublishing.org/doi/pdf/10.1098/rsta.1977.0145.

[97] M. Berry, Some quantum-to-classical asymptotics, In Chaos and Quantum Physics: Proceedings of the Les Houches Summer School, Course LII, pp. 251 - 304, ISBN 04448277X (1991).

[98] M. V. Berry, M. Tabor and J. M. Ziman, Level clustering in the regular spectrum, Proceedings of the Royal Society of London. A. Mathematical and Physical Sciences 356(1686), 375 (1977), doi:10.1098/rspa.1977.0140, https://royalsocietypublishing.org/doi/pdf/10. 1098/rspa.1977.0140.

[99] P. Pechukas, Distribution of energy eigenvalues in the irregular spectrum, Phys. Rev. Lett. 51, 943 (1983), doi:10.1103/PhysRevLett.51.943.

[100] O. Bohigas, M. J. Giannoni and C. Schmit, Characterization of chaotic quantum spectra and universality of level fluctuation laws, Phys. Rev. Lett. 52, 1 (1984), doi:10.1103/PhysRevLett. 52.1 .

[101] Bohigas, O., Giannoni, M.J. and Schmit, C., Spectral properties of the laplacian and random matrix theories, J. Physique Lett. 45(21), 1015 (1984), doi:10.1051/jphyslet: 0198400450210101500 .

[102] M. V. Berry and M. Robnik, Semiclassical level spacings when regular and chaotic orbits coexist, Journal of Physics A: Mathematical and General 17(12), 2413 (1984), doi:10.1088/ 0305-4470/17/12/013.

[103] M. V. Berry, I. C. Percival and N. O. Weiss, The bakerian lecture, 1987. quantum chaology, Proceedings of the Royal Society of London. A. Mathematical and Physical Sciences 413(1844), 183 (1987), doi:10.1098/rspa.1987.0109, https://royalsocietypublishing. org/doi/pdf/10.1098/rspa.1987.0109.

[104] A. N. Kolmogorov, On conservation of conditionally periodic motions for a small change in Hamilton's function, Dokl. Akad. Nauk SSSR (N.S.) 98, 527 (1954).

[105] V. I. Arnold, Proof of a theorem of A. N. Kolmogorov on the preservation of conditionally periodic motions under a small perturbation of the Hamiltonian, Uspehi Mat. Nauk 18(5 (113)), 13 (1963). 
Bibliography

[106] J. Moser, On invariant curves of area-preserving mappings of an annulus, Nachr. Akad. Wiss. Göttingen Math.-Phys. Kl. II 1962, 1 (1962).

[107] J. H. Hannay and A. M. O. D. Almeida, Periodic orbits and a correlation function for the semiclassical density of states, Journal of Physics A: Mathematical and General 17(18), 3429 (1984), doi:10.1088/0305-4470/17/18/013.

[108] M. V. Berry, Semiclassical theory of spectral rigidity, Proceedings of the Royal Society of London. A. Mathematical and Physical Sciences 400(1819), 229 (1985), doi:10.1098/rspa. 1985.0078, https://royalsocietypublishing.org/doi/pdf/10.1098/rspa.1985.0078.

[109] M. C. Gutzwiller, Periodic orbits and classical quantization conditions, Journal of Mathematical Physics 12(3), 343 (1971), doi:10.1063/1.1665596, https://doi.org/10.1063/1. 1665596 .

[110] M. C. Gutzwiller, Path integrals and the relation between classical and quantum mechanics, In G. Papadopoulos and J. Devreese, eds., Path integrals and their applications in quantum, statistical, and solid state physics, vol. 34 of NATO advanced study institutes series. Springer, Boston, MA, ISBN 978-1-4684-9142-5 (1978).

[111] R. Balian and C. Bloch, Distribution of eigenfrequencies for the wave equation in a finite domain: Iii. eigenfrequency density oscillations, Annals of Physics 69(1), 76 (1972), doi: https://doi.org/10.1016/0003-4916(72)90006-1.

[112] R. Balian and C. Bloch, Solution of the schroedinger equation in terms of classical paths, Annals of Physics 85(2), 514 (1974), doi:https://doi.org/10.1016/0003-4916(74)90421-7.

[113] M. V. Berry, M. Tabor and J. M. Ziman, Closed orbits and the regular bound spectrum, Proceedings of the Royal Society of London. A. Mathematical and Physical Sciences 349(1656), 101 (1976), doi:10.1098/rspa.1976.0062, https://royalsocietypublishing.org/doi/pdf/ 10.1098/rspa.1976.0062.

[114] E. J. Heller, Bound-state eigenfunctions of classically chaotic hamiltonian systems: Scars of periodic orbits, Phys. Rev. Lett. 53, 1515 (1984), doi:10.1103/PhysRevLett.53.1515.

[115] E. J. Heller, Qualitative properties of eigenfunctions of classically chaotic hamiltonian systems, In T. H. Seligman and H. Nishioka, eds., Quantum Chaos and Statistical Nuclear Physics, pp. 162-181. Springer Berlin Heidelberg, Berlin, Heidelberg, ISBN 978-3-540-472308 (1986).

[116] E. J. Heller and P. W. O'Connor, The quest for quantum chaos in the stadium, Nuclear Physics B - Proceedings Supplements 2, 201 (1987), doi:https://doi.org/10.1016/ 0920-5632(87)90018-1.

[117] M. V. Berry, Quantum scars of classical closed orbits in phase space, Proceedings of the Royal Society of London. A. Mathematical and Physical Sciences 423(1864), 219 (1989), doi:10.1098/rspa.1989.0052, https://royalsocietypublishing.org/doi/pdf/10.1098/ rspa.1989.0052.

[118] Y. Sinai, Introduction to Ergodic Theory, Mathematical Notes - Princeton University Press. Princeton University Press, ISBN 9780691081823 (1977).

[119] M. Berry, Quantizing a classically ergodic system: Sinai's billiard and the kkr method, Annals of Physics 131(1), 163 (1981), doi:https://doi.org/10.1016/0003-4916(81)90189-5. 
[120] A. Einstein, Zum quantensatz von sommerfeld und epstein, In J. J. e. a. Stachel, ed., The collected papers of Albert Einstein: English translation supplement, vol. 6, pp. $434-444$. Princeton Univ. Press, Princeton, NJ, ISBN 978-0691017341 (1917).

[121] C. Porter, Statistical Theories of Spectra: Fluctuations, Perspectives in Physics Series. Academic Press, ISBN 9780125623568 (1965).

[122] M. Robnik and H. H. Wills, Antiunitary symmetries and energy level statistics, In T. H. Seligman and H. Nishioka, eds., Quantum Chaos and Statistical Nuclear Physics, pp. 120130. Springer Berlin Heidelberg, Berlin, Heidelberg, ISBN 978-3-540-47230-8 (1986).

[123] L. Erdos, Universality of wigner random matrices: a survey of recent results, Russian Mathematical Surveys 66(3), 507 (2011), doi:10.1070/rm2011v066n03abeh004749.

[124] M. V. Berry, Aspects of degeneracy, In G. Casati, ed., Chaotic Behavior in Quantum Systems: Theory and Applications, pp. 123-140. Springer US, Boston, MA, ISBN 978-1-4613-2443-0, doi:10.1007/978-1-4613-2443-0_8 (1985).

[125] C. Chester, B. Friedman and F. Ursell, An extension of the method of steepest descents, Mathematical Proceedings of the Cambridge Philosophical Society 53(3), 599 (1957), doi: 10.1017/S0305004100032655.

[126] M. V. Berry and K. E. Mount, Semiclassical approximations in wave mechanics, Reports on Progress in Physics 35(1), 315 (1972), doi:10.1088/0034-4885/35/1/306.

[127] M. V. Berry, Evolution of semiclassical quantum states in phase space, Journal of Physics A: Mathematical and General 12(5), 625 (1979), doi:10.1088/0305-4470/12/5/012.

[128] E. J. Heller, Wavepacket path integral formulation of semiclassical dynamics, Chemical Physics Letters 34(2), 321 (1975), doi:http://dx.doi.org/10.1016/0009-2614(75)85284-5.

[129] J. H. V. Vleck, The correspondence principle in the statistical interpretation of quantum mechanics, Proceedings of the National Academy of Sciences of the United States of America 14(2), 178 (1928).

[130] J. B. Keller, Corrected Bohr-Sommerfeld quantum conditions for nonseparable systems, Annals of Physics 4(2), 180 (1958).

[131] C. Bender and S. Orszag, Advanced Mathematical Methods for Scientists and Engineers I: Asymptotic Methods and Perturbation Theory, Advanced Mathematical Methods for Scientists and Engineers. Springer, ISBN 9780387989310 (1999).

[132] M. de Gosson, Symplectic Geometry and Quantum Mechanics, Operator Theory: Advances and Applications. Birkhäuser Basel, ISBN 9783764375751 (2006).

[133] M. de Gosson, Symplectic Methods in Harmonic Analysis and in Mathematical Physics, Pseudo-Differential Operators. Springer Basel, ISBN 9783764399924 (2011).

[134] F. Bayen, M. Flato, C. Fronsdal, A. Lichnerowicz and D. Sternheimer, Quantum mechanics as a deformation of classical mechanics, Letters in Mathematical Physics 1(6), 521 (1977), doi:10.1007/BF00399745.

[135] T. Curtright, T. Uematsu and C. Zachos, Generating all wigner functions, Journal of Mathematical Physics 42(6), 2396 (2001), doi:10.1063/1.1366327, https://doi.org/10. 1063/1.1366327. 
Bibliography

[136] A. S. Holevo, M. Sohma and O. Hirota, Capacity of quantum gaussian channels, Phys. Rev. A 59, 1820 (1999), doi:10.1103/PhysRevA.59.1820.

[137] M. Krbek, T. Tyc and J. Vlach, Inequalities for quantum marginal problems with continuous variables, Journal of Mathematical Physics 55(6), 062201 (2014), doi:10.1063/1.4880198, https://doi.org/10.1063/1.4880198.

[138] M. Gromov, Pseudo holomorphic curves in symplectic manifolds, Inventiones mathematicae 82(2), 307 (1985), doi:10.1007/BF01388806.

[139] I. Stewart, The symplectic camel, Nature 329(6134), 17 (1987), doi:10.1038/329017a0.

[140] M. A. de Gosson, The symplectic camel and the uncertainty principle: The tip of an iceberg?, Foundations of Physics 39(2), 194 (2009), doi:10.1007/s10701-009-9272-2.

[141] J. Eisert, T. Tyc, T. Rudolph and B. C. Sanders, Gaussian quantum marginal problem, Communications in Mathematical Physics 280(1), 263 (2008), doi:10.1007/s00220-008-0442-4.

[142] T. Cover and J. Thomas, Elements of Information Theory, Wiley, ISBN 9781118585771 (2012).

[143] F. Bayen, M. Flato, C. Fronsdal, A. Lichnerowicz and D. Sternheimer, Deformation theory and quantization. ii. physical applications, Annals of Physics 111(1), 111 (1978), doi:https: //doi.org/10.1016/0003-4916(78)90225-7.

[144] A. Wehrl, On the relation between classical and quantum-mechanical entropy, Reports on Mathematical Physics 16(3), 353 (1979), doi:https://doi.org/10.1016/0034-4877(79)90070-3.

[145] P. Hosur, X.-L. Qi, D. A. Roberts and B. Yoshida, Chaos in quantum channels, Journal of High Energy Physics 2016(2), 4 (2016), doi:10.1007/JHEP02(2016)004.

[146] O. Schnaack, N. Bölter, S. Paeckel, S. R. Manmana, S. Kehrein and M. Schmitt, Tripartite information, scrambling, and the role of hilbert space partitioning in quantum lattice models, Phys. Rev. B 100, 224302 (2019), doi:10.1103/PhysRevB.100.224302.

[147] R. Oppenheimer, Analogy in science, The Centennial Review of Arts \& Science 2, 351 (1958).

[148] J. C. Halimeh and V. Zauner-Stauber, Dynamical phase diagram of quantum spin chains with long-range interactions, Phys. Rev. B 96, 134427 (2017), doi:10.1103/PhysRevB.96.134427.

[149] B. Zunkovic, A. Silva and M. Fabrizio, Dynamical phase transitions and loschmidt echo in the infinite-range xy model, Philosophical Transactions of the Royal Society A: Mathematical, Physical and Engineering Sciences 374(2069), 20150160 (2016), doi:10.1098/rsta.2015.0160, https://royalsocietypublishing.org/doi/pdf/10.1098/rsta.2015.0160

[150] J. Lang, B. Frank and J. C. Halimeh, Dynamical quantum phase transitions: A geometric picture, Phys. Rev. Lett. 121, 130603 (2018), doi:10.1103/PhysRevLett.121.130603.

[151] W. van Saarloos and D. A. Kurtze, Location of zeros in the complex temperature plane: absence of lee-yang theorem, Journal of Physics A: Mathematical and General 17(6), 1301 (1984), doi:10.1088/0305-4470/17/6/026.

[152] A. Silva, Statistics of the work done on a quantum critical system by quenching a control parameter, Phys. Rev. Lett. 101, 120603 (2008), doi:10.1103/PhysRevLett.101.120603. 
[153] S. Vajna and B. Dóra, Disentangling dynamical phase transitions from equilibrium phase transitions, Phys. Rev. B 89, 161105 (2014), doi:10.1103/PhysRevB.89.161105.

[154] S. Vajna and B. Dóra, Disentangling dynamical phase transitions from equilibrium phase transitions, Phys. Rev. B 89, 161105 (2014), doi:10.1103/PhysRevB.89.161105.

[155] M. Heyl, Dynamical quantum phase transitions in systems with broken-symmetry phases, Phys. Rev. Lett. 113, 205701 (2014), doi:10.1103/PhysRevLett.113.205701.

[156] T. Pálmai and S. Sotiriadis, Quench echo and work statistics in integrable quantum field theories, Phys. Rev. E 90, 052102 (2014), doi:10.1103/PhysRevE.90.052102.

[157] S. Vajna and B. Dóra, Topological classification of dynamical phase transitions, Phys. Rev. B 91, 155127 (2015), doi:10.1103/PhysRevB.91.155127.

[158] J. C. Budich and M. Heyl, Dynamical topological order parameters far from equilibrium, Phys. Rev. B 93, 085416 (2016), doi:10.1103/PhysRevB.93.085416.

[159] C. Karrasch and D. Schuricht, Dynamical phase transitions after quenches in nonintegrable models, Phys. Rev. B 87, 195104 (2013), doi:10.1103/PhysRevB.87.195104.

[160] J. N. Kriel, C. Karrasch and S. Kehrein, Dynamical quantum phase transitions in the axial next-nearest-neighbor ising chain, Phys. Rev. B 90, 125106 (2014), doi:10.1103/PhysRevB. 90.125106

[161] A. Lerose, B. Žunkovič, J. Marino, A. Gambassi and A. Silva, Impact of nonequilibrium fluctuations on prethermal dynamical phase transitions in long-range interacting spin chains, Phys. Rev. B 99, 045128 (2019), doi:10.1103/PhysRevB.99.045128.

[162] M. Schmitt and S. Kehrein, Dynamical quantum phase transitions in the kitaev honeycomb model, Phys. Rev. B 92, 075114 (2015), doi:10.1103/PhysRevB.92.075114.

[163] M. Heyl, Scaling and universality at dynamical quantum phase transitions, Phys. Rev. Lett. 115, 140602 (2015), doi:10.1103/PhysRevLett.115.140602.

[164] S. A. Weidinger, M. Heyl, A. Silva and M. Knap, Dynamical quantum phase transitions in systems with continuous symmetry breaking, Phys. Rev. B 96, 134313 (2017), doi:10.1103/ PhysRevB.96.134313.

[165] J. Lang, B. Frank and J. C. Halimeh, Concurrence of dynamical phase transitions at finite temperature in the fully connected transverse-field ising model, Phys. Rev. B 97, 174401 (2018), doi:10.1103/PhysRevB.97.174401.

[166] N. O. Abeling and S. Kehrein, Quantum quench dynamics in the transverse field ising model at nonzero temperatures, Phys. Rev. B 93, 104302 (2016), doi:10.1103/PhysRevB.93.104302.

[167] S. Bandyopadhyay, S. Laha, U. Bhattacharya and A. Dutta, Exploring the possibilities of dynamical quantum phase transitions in the presence of a markovian bath, Scientific Reports 8(1), 11921 (2018), doi:10.1038/s41598-018-30377-x.

[168] R. Jafari, Dynamical quantum phase transition and quasi particle excitation, Scientific Reports 9(1), 2871 (2019), doi:10.1038/s41598-019-39595-3.

[169] M. Berry, N. Balazs, M. Tabor and A. Voros, Quantum maps, Annals of Physics 122(1), 26 (1979), doi:https://doi.org/10.1016/0003-4916(79)90296-3. 
Bibliography

[170] Y. Meyer, Quasicrystals, diophantine approximation and algebraic numbers, In F. Axel and D. Gratias, eds., Beyond Quasicrystals, pp. 3-16. Springer Berlin Heidelberg, Berlin, Heidelberg, ISBN 978-3-662-03130-8 (1995).

[171] Tao, Terence, The uncertainty principle, https://terrytao.wordpress.com/2010/06/25/ the-uncertainty-principle/, [Online; accessed 29-January-2020] (2010).

[172] R. Ellis, Entropy, Large Deviations, and Statistical Mechanics, Classics in Mathematics. Springer, ISBN 978-3-540-29059-9, doi:10.1007/3-540-29060-5 (2006).

[173] R. Ellis, The theory of large deviations and applications to statistical mechanics (2009).

[174] R. Bellman, A brief introduction to theta functions, Athena series selected topics in mathematics. Holt, Rinehart and Winston (1961).

[175] T. Curtright, D. Fairlie and C. Zachos, Quantum Mechanics In Phase Space: An Overview With Selected Papers, World Scientific Series In 20th Century Physics. World Scientific Publishing Company, ISBN 9789814485876 (2005).

[176] J. N. Kriel and F. G. Scholtz, Eigenvalue distributions from a star product approach, Journal of Physics A: Mathematical and Theoretical 45(47), 475204 (2012), doi:10.1088/1751-8113/ $45 / 47 / 475204$.

[177] D. N. Page and W. K. Wootters, Evolution without evolution: Dynamics described by stationary observables, Phys. Rev. D 27, 2885 (1983), doi:10.1103/PhysRevD.27.2885.

[178] E. Schrödinger, Die gegenwärtige situation in der quantenmechanik, Naturwissenschaften 23(50), 844 (1935), doi:10.1007/BF01491987.

[179] V. I. Arnold and A. Avez, Ergodic Problems of Classical Mechanics, Advanced book classics. Addison-Wesley, ISBN 9780201094060 (1989).

[180] E. T. Jaynes, Gibbs vs boltzmann entropies (1965), In R. D. Rosenkrantz, ed., E. T. Jaynes: Papers on Probability, Statistics and Statistical Physics, pp. 77-86. Springer Netherlands, Dordrecht, ISBN 978-94-009-6581-2, doi:10.1007/978-94-009-6581-2_5 (1989).

[181] A. S. Holevo, Bounds for the quantity of information transmitted by a quantum communication channel, Problems of Information Transmission 9, 177 (1973).

[182] L. Mandelstam and I. Tamm, The uncertainty relation between energy and time in nonrelativistic quantum mechanics, In B. M. Bolotovskii, V. Y. Frenkel and R. Peierls, eds., Selected Papers, pp. 115-123. Springer Berlin Heidelberg, Berlin, Heidelberg, ISBN 978-3642-74626-0 (1991).

[183] N. Margolus and L. B. Levitin, The maximum speed of dynamical evolution, Physica D: Nonlinear Phenomena 120(1), 188 (1998), doi:https://doi.org/10.1016/S0167-2789(98)00054-2, Proceedings of the Fourth Workshop on Physics and Consumption.

[184] V. Giovannetti, S. Lloyd and L. Maccone, Quantum time, Phys. Rev. D 92, 045033 (2015), doi:10.1103/PhysRevD.92.045033.

[185] A. Boette, R. Rossignoli, N. Gigena and M. Cerezo, System-time entanglement in a discretetime model, Phys. Rev. A 93, 062127 (2016), doi:10.1103/PhysRevA.93.062127. 
[186] R. P. Feynman, Quantum mechanical computers, Foundations of Physics 16(6), 507 (1986), doi:10.1007/BF01886518.

[187] L. Mandelstam and I. Tamm, The uncertainty relation between energy and time in nonrelativistic quantum mechanics, J. Phys. USSR 9, 249 (1945).

[188] V. Giovannetti, S. Lloyd and L. Maccone, Achieving the holevo bound via sequential measurements, Phys. Rev. A 85, 012302 (2012), doi:10.1103/PhysRevA.85.012302.

[189] J. Flores, M. Horoi, M. Müller and T. H. Seligman, Spectral statistics of the two-body random ensemble revisited, Phys. Rev. E 63, 026204 (2001), doi:10.1103/PhysRevE.63.026204.

[190] L. F. Santos and M. Rigol, Onset of quantum chaos in one-dimensional bosonic and fermionic systems and its relation to thermalization, Phys. Rev. E 81, 036206 (2010), doi:10.1103/ PhysRevE.81.036206.

[191] M. L. Mehta, Random Matrices, Elsevier Science, 3rd edn., ISBN 9780080474113 (2004).

[192] L. Erdos and H.-T. Yau, Universality of local spectral statistics of random matrices, Bull. Amer. Math. Soc. 49(3), 377 (2012), doi:https://doi.org/10.1090/S0273-0979-2012-01372-1.

[193] L. Erdos and H. Yau, A Dynamical Approach to Random Matrix Theory, Courant Lecture Notes. Courant Institute of Mathematical Sciences, New York University, ISBN 9781470436483 (2017).

[194] F. H. L. Essler, H. Frahm, F. Göhmann, A. Klümper and V. E. Korepin, The OneDimensional Hubbard Model, Cambridge University Press, doi:10.1017/CBO9780511534843 (2005).

[195] A. W. Sandvik, Computational studies of quantum spin systems, AIP Conference Proceedings 1297(1), 135 (2010), doi:10.1063/1.3518900, https://aip.scitation.org/doi/pdf/10. $1063 / 1.3518900$.

[196] F. Verhulst, Nonlinear Differential Equations and Dynamical Systems, Universitext. Springer Berlin Heidelberg, ISBN 9783642614538 (2012).

[197] N. Bölter et al., unpublished, Unpublished. 